\title{
One-pot Synthesis of 1, 5-Diketones under Transition-Metal-free Condition: Application in the Synthesis of 2,4,6-Triaryl Pyridine Derivatives
}

\author{
Kesatebrhan Haile Asressu, Chieh-Kai Chan, Cheng-Chung Wang* \\ Institute of Chemistry, Academia Sinica Taipei 115, Taiwan \\ E-mail: wangcc@chem.sinica.edu.tw
}

I. Crystal data for compounds 3o, 3q, 3t, $\mathbf{4 e}$, and $\mathbf{4 r} \ldots \ldots \ldots \ldots \ldots \ldots \ldots \ldots . . .2-\mathrm{S}-47$

II. $\quad{ }^{1} \mathrm{H}$ and ${ }^{13} \mathrm{C}$ NMR spectra for compounds 3a-t.......................... $-48 \sim \mathrm{S}-87$

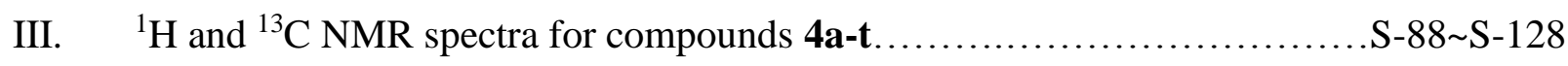

IV. $\quad{ }^{1} \mathrm{H}$ and ${ }^{13} \mathrm{C}$ NMR spectra for compounds 5a-e.......................S-129 S-137 


\section{Crystal data and structure refinement for compound 3o}

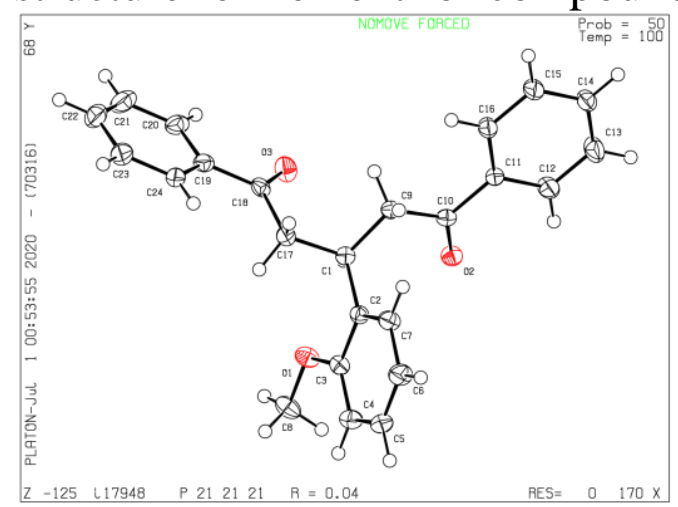

Figure S1. Single X-ray crystal structure of 1, 5-dicarbonyls 30 (the thermal ellipsoid was drawn at the 50\% probability level)

Table S1. Crystal data and structure refinement for i17948

\begin{tabular}{|c|c|c|}
\hline $\mathrm{CCDC}$ & \multicolumn{2}{|l|}{2031225} \\
\hline Identification code & \multicolumn{2}{|l|}{ i17948 } \\
\hline Empirical formula & \multicolumn{2}{|l|}{$\mathrm{C} 24 \mathrm{H} 22 \mathrm{O} 3$} \\
\hline Formula weight & \multicolumn{2}{|l|}{358.41} \\
\hline Temperature & \multicolumn{2}{|l|}{$100.0(2) \mathrm{K}$} \\
\hline Wavelength & \multicolumn{2}{|l|}{$1.54178 \AA$} \\
\hline Crystal system & \multicolumn{2}{|l|}{ Orthorhombic } \\
\hline Space group & \multicolumn{2}{|l|}{ P 212121} \\
\hline \multirow[t]{3}{*}{ Unit cell dimensions } & $\mathrm{a}=5.2961(2) \AA$ & $\alpha=90^{\circ}$. \\
\hline & $\mathrm{b}=17.1066(5) \AA$ & $\beta=90^{\circ}$. \\
\hline & $c=20.7005(6) \AA$ & $\gamma=90^{\circ}$ \\
\hline Volume & \multicolumn{2}{|l|}{$1875.43(10) \AA^{3}$} \\
\hline Z, Density (calculated) & \multicolumn{2}{|l|}{$4,1.269 \mathrm{Mg} / \mathrm{m}^{3}$} \\
\hline Absorption coefficient & \multicolumn{2}{|l|}{$0.658 \mathrm{~mm}^{-1}$} \\
\hline $\mathrm{F}(000)$ & \multicolumn{2}{|l|}{760} \\
\hline Crystal size & \multicolumn{2}{|c|}{$0.272 \times 0.156 \times 0.116 \mathrm{~mm}^{3}$} \\
\hline Theta range for data collection & \multicolumn{2}{|l|}{3.351 to $66.664^{\circ}$. } \\
\hline Index ranges & \multicolumn{2}{|c|}{$-6<=\mathrm{h}<=5,-20<=\mathrm{k}<=20,-24<=1<=24$} \\
\hline Reflections collected & \multicolumn{2}{|l|}{13354} \\
\hline Independent reflections & \multicolumn{2}{|c|}{$3308[\mathrm{R}(\mathrm{int})=0.0638]$} \\
\hline Completeness to theta $=66.664^{\circ}$ & \multicolumn{2}{|l|}{$99.6 \%$} \\
\hline Absorption correction & \multicolumn{2}{|l|}{ Numerical } \\
\hline Max. and min. transmission & \multicolumn{2}{|l|}{1 and 0.7968} \\
\hline Refinement method & \multicolumn{2}{|c|}{ Full-matrix least-squares on $\mathrm{F}^{2}$} \\
\hline Data / restraints / parameters & \multicolumn{2}{|l|}{$3308 / 0 / 249$} \\
\hline Goodness-of-fit on $\mathrm{F}^{2}$ & \multicolumn{2}{|l|}{1.056} \\
\hline Final $\mathrm{R}$ indices $[\mathrm{I}>2 \operatorname{sigma}(\mathrm{I})]$ & \multicolumn{2}{|c|}{$\mathrm{R} 1=0.0353, \mathrm{wR} 2=0.0855$} \\
\hline
\end{tabular}


$\mathrm{R}$ indices (all data)

Absolute structure parameter

Extinction coefficient

Largest diff. peak and hole
$\mathrm{R} 1=0.0397, \mathrm{wR} 2=0.0879$

$0.4(3)$

$\mathrm{n} / \mathrm{a}$

0.146 and -0.151 e. $\AA^{-3}$

Table S2. Atomic coordinates ( $\left.x \mathbf{1 0}^{4}\right)$ and equivalent isotropic displacement parameters $\left(\AA^{2} \times 1^{3}\right)$ for i17948. $U(\mathrm{eq})$ is defined as one third of the trace of the orthogonalized $U^{i j}$ tensor.

\begin{tabular}{|c|c|c|c|c|}
\hline & $\mathrm{X}$ & $\mathrm{y}$ & z & $\mathrm{U}(\mathrm{eq})$ \\
\hline $\mathrm{O}(1)$ & $66(3)$ & $3522(1)$ & $4372(1)$ & $25(1)$ \\
\hline $\mathrm{O}(2)$ & $-166(3)$ & $5877(1)$ & $4024(1)$ & $23(1)$ \\
\hline $\mathrm{O}(3)$ & $2518(3)$ & $5121(1)$ & $5904(1)$ & $30(1)$ \\
\hline $\mathrm{C}(1)$ & $3104(4)$ & $4808(1)$ & $4595(1)$ & $19(1)$ \\
\hline $\mathrm{C}(2)$ & $3220(4)$ & $4357(1)$ & 3961(1) & $19(1)$ \\
\hline $\mathrm{C}(3)$ & $1636(4)$ & $3711(1)$ & $3865(1)$ & $20(1)$ \\
\hline $\mathrm{C}(4)$ & $1720(4)$ & $3281(1)$ & $3295(1)$ & $25(1)$ \\
\hline$C(5)$ & $3465(5)$ & $3485(1)$ & $2816(1)$ & $26(1)$ \\
\hline$C(6)$ & $5083(5)$ & $4106(1)$ & $2906(1)$ & $29(1)$ \\
\hline $\mathrm{C}(7)$ & $4932(5)$ & $4539(1)$ & $3479(1)$ & $24(1)$ \\
\hline $\mathrm{C}(8)$ & $-1625(4)$ & $2887(1)$ & $4282(1)$ & $29(1)$ \\
\hline $\mathrm{C}(9)$ & $3822(4)$ & $5669(1)$ & $4528(1)$ & $18(1)$ \\
\hline$C(10)$ & $1864(4)$ & $6156(1)$ & $4186(1)$ & $18(1)$ \\
\hline $\mathrm{C}(11)$ & $2421(4)$ & 7001(1) & 4064(1) & $18(1)$ \\
\hline$C(12)$ & $754(5)$ & $7431(1)$ & $3684(1)$ & $30(1)$ \\
\hline$C(13)$ & $1168(5)$ & $8225(1)$ & $3576(2)$ & $36(1)$ \\
\hline$C(14)$ & $3249(4)$ & $8591(1)$ & $3843(1)$ & $26(1)$ \\
\hline$C(15)$ & $4918(5)$ & $8167(1)$ & $4218(1)$ & $30(1)$ \\
\hline$C(16)$ & $4511(4)$ & $7370(1)$ & $4326(1)$ & $26(1)$ \\
\hline$C(17)$ & $4771(4)$ & $4411(1)$ & $5099(1)$ & $20(1)$ \\
\hline$C(18)$ & $4234(4)$ & $4668(1)$ & $5786(1)$ & $20(1)$ \\
\hline$C(19)$ & $5787(4)$ & $4342(1)$ & $6321(1)$ & $22(1)$ \\
\hline$C(20)$ & $5006(5)$ & $4486(1)$ & $6956(1)$ & $28(1)$ \\
\hline $\mathrm{C}(21)$ & $6358(5)$ & $4182(2)$ & $7471(1)$ & $38(1)$ \\
\hline $\mathrm{C}(22)$ & $8505(5)$ & $3735(2)$ & $7363(1)$ & $37(1)$ \\
\hline$C(23)$ & $9300(5)$ & $3585(2)$ & $6733(1)$ & $33(1)$ \\
\hline $\mathrm{C}(24)$ & $7939(5)$ & $3888(1)$ & $6216(1)$ & $24(1)$ \\
\hline
\end{tabular}


Table S3. Bond lengths $[\AA ̊]$ and angles $\left[{ }^{\circ}\right]$ for $\mathbf{i l 9 4 8}$.

\begin{tabular}{|c|c|}
\hline $\mathrm{O}(1)-\mathrm{C}(3)$ & $1.378(3)$ \\
\hline $\mathrm{O}(1)-\mathrm{C}(8)$ & $1.420(3)$ \\
\hline $\mathrm{O}(2)-\mathrm{C}(10)$ & $1.224(3)$ \\
\hline $\mathrm{O}(3)-\mathrm{C}(18)$ & $1.220(3)$ \\
\hline $\mathrm{C}(1)-\mathrm{C}(2)$ & $1.522(3)$ \\
\hline $\mathrm{C}(1)-\mathrm{C}(17)$ & $1.527(3)$ \\
\hline $\mathrm{C}(1)-\mathrm{C}(9)$ & $1.527(3)$ \\
\hline $\mathrm{C}(1)-\mathrm{H}(1)$ & 1.0000 \\
\hline$C(2)-C(7)$ & $1.385(3)$ \\
\hline $\mathrm{C}(2)-\mathrm{C}(3)$ & $1.401(3)$ \\
\hline $\mathrm{C}(3)-\mathrm{C}(4)$ & $1.391(3)$ \\
\hline$C(4)-C(5)$ & $1.400(3)$ \\
\hline $\mathrm{C}(4)-\mathrm{H}(4)$ & 0.9500 \\
\hline$C(5)-C(6)$ & $1.378(3)$ \\
\hline $\mathrm{C}(5)-\mathrm{H}(5)$ & 0.9500 \\
\hline$C(6)-C(7)$ & $1.399(3)$ \\
\hline $\mathrm{C}(6)-\mathrm{H}(6)$ & 0.9500 \\
\hline $\mathrm{C}(7)-\mathrm{H}(7)$ & 0.9500 \\
\hline $\mathrm{C}(8)-\mathrm{H}(8 \mathrm{~A})$ & 0.9800 \\
\hline $\mathrm{C}(8)-\mathrm{H}(8 \mathrm{~B})$ & 0.9800 \\
\hline $\mathrm{C}(8)-\mathrm{H}(8 \mathrm{C})$ & 0.9800 \\
\hline $\mathrm{C}(9)-\mathrm{C}(10)$ & $1.506(3)$ \\
\hline $\mathrm{C}(9)-\mathrm{H}(9 \mathrm{~A})$ & 0.9900 \\
\hline $\mathrm{C}(9)-\mathrm{H}(9 \mathrm{AB})$ & 0.9900 \\
\hline $\mathrm{C}(10)-\mathrm{C}(11)$ & $1.497(3)$ \\
\hline$C(11)-C(16)$ & $1.385(3)$ \\
\hline $\mathrm{C}(11)-\mathrm{C}(12)$ & $1.393(3)$ \\
\hline $\mathrm{C}(12)-\mathrm{C}(13)$ & $1.394(3)$ \\
\hline $\mathrm{C}(12)-\mathrm{H}(12)$ & 0.9500 \\
\hline$C(13)-C(14)$ & $1.383(3)$ \\
\hline $\mathrm{C}(13)-\mathrm{H}(13)$ & 0.9500 \\
\hline$C(14)-C(15)$ & $1.383(3)$ \\
\hline $\mathrm{C}(14)-\mathrm{H}(14)$ & 0.9500 \\
\hline$C(15)-C(16)$ & $1.399(3)$ \\
\hline $\mathrm{C}(15)-\mathrm{H}(15)$ & 0.9500 \\
\hline $\mathrm{C}(16)-\mathrm{H}(16)$ & 0.9500 \\
\hline
\end{tabular}




\begin{tabular}{|c|c|}
\hline $\mathrm{C}(17)-\mathrm{C}(18)$ & $1.515(3)$ \\
\hline $\mathrm{C}(17)-\mathrm{H}(17 \mathrm{~A})$ & 0.9900 \\
\hline C(17)-H(17B) & 0.9900 \\
\hline C(18)-C(19) & $1.487(3)$ \\
\hline C(19)-C(24) & $1.396(3)$ \\
\hline C(19)-C(20) & $1.401(3)$ \\
\hline $\mathrm{C}(20)-\mathrm{C}(21)$ & $1.386(4)$ \\
\hline $\mathrm{C}(20)-\mathrm{H}(20)$ & 0.9500 \\
\hline $\mathrm{C}(21)-\mathrm{C}(22)$ & $1.389(4)$ \\
\hline $\mathrm{C}(21)-\mathrm{H}(21)$ & 0.9500 \\
\hline $\mathrm{C}(22)-\mathrm{C}(23)$ & $1.395(4)$ \\
\hline $\mathrm{C}(22)-\mathrm{H}(22)$ & 0.9500 \\
\hline C(23)-C(24) & $1.390(3)$ \\
\hline $\mathrm{C}(23)-\mathrm{H}(23)$ & 0.9500 \\
\hline $\mathrm{C}(24)-\mathrm{H}(24)$ & 0.9500 \\
\hline $\mathrm{C}(3)-\mathrm{O}(1)-\mathrm{C}(8)$ & $117.37(18)$ \\
\hline $\mathrm{C}(2)-\mathrm{C}(1)-\mathrm{C}(17)$ & $109.86(17)$ \\
\hline $\mathrm{C}(2)-\mathrm{C}(1)-\mathrm{C}(9)$ & $113.65(18)$ \\
\hline $\mathrm{C}(17)-\mathrm{C}(1)-\mathrm{C}(9)$ & $110.28(18)$ \\
\hline $\mathrm{C}(2)-\mathrm{C}(1)-\mathrm{H}(1)$ & 107.6 \\
\hline $\mathrm{C}(17)-\mathrm{C}(1)-\mathrm{H}(1)$ & 107.6 \\
\hline $\mathrm{C}(9)-\mathrm{C}(1)-\mathrm{H}(1)$ & 107.6 \\
\hline $\mathrm{C}(7)-\mathrm{C}(2)-\mathrm{C}(3)$ & $117.8(2)$ \\
\hline $\mathrm{C}(7)-\mathrm{C}(2)-\mathrm{C}(1)$ & $122.25(19)$ \\
\hline $\mathrm{C}(3)-\mathrm{C}(2)-\mathrm{C}(1)$ & $119.90(19)$ \\
\hline $\mathrm{O}(1)-\mathrm{C}(3)-\mathrm{C}(4)$ & $122.82(19)$ \\
\hline $\mathrm{O}(1)-\mathrm{C}(3)-\mathrm{C}(2)$ & $115.93(19)$ \\
\hline $\mathrm{C}(4)-\mathrm{C}(3)-\mathrm{C}(2)$ & 121.2(2) \\
\hline $\mathrm{C}(3)-\mathrm{C}(4)-\mathrm{C}(5)$ & $119.4(2)$ \\
\hline $\mathrm{C}(3)-\mathrm{C}(4)-\mathrm{H}(4)$ & 120.3 \\
\hline $\mathrm{C}(5)-\mathrm{C}(4)-\mathrm{H}(4)$ & 120.3 \\
\hline$C(6)-C(5)-C(4)$ & $120.4(2)$ \\
\hline $\mathrm{C}(6)-\mathrm{C}(5)-\mathrm{H}(5)$ & 119.8 \\
\hline $\mathrm{C}(4)-\mathrm{C}(5)-\mathrm{H}(5)$ & 119.8 \\
\hline$C(5)-C(6)-C(7)$ & 119.2(2) \\
\hline $\mathrm{C}(5)-\mathrm{C}(6)-\mathrm{H}(6)$ & 120.4 \\
\hline $\mathrm{C}(7)-\mathrm{C}(6)-\mathrm{H}(6)$ & 120.4 \\
\hline$C(2)-C(7)-C(6)$ & $122.0(2)$ \\
\hline
\end{tabular}




\begin{tabular}{|c|c|}
\hline $\mathrm{C}(2)-\mathrm{C}(7)-\mathrm{H}(7)$ & 119.0 \\
\hline $\mathrm{C}(6)-\mathrm{C}(7)-\mathrm{H}(7)$ & 119.0 \\
\hline $\mathrm{O}(1)-\mathrm{C}(8)-\mathrm{H}(8 \mathrm{~A})$ & 109.5 \\
\hline $\mathrm{O}(1)-\mathrm{C}(8)-\mathrm{H}(8 \mathrm{~B})$ & 109.5 \\
\hline $\mathrm{H}(8 \mathrm{~A})-\mathrm{C}(8)-\mathrm{H}(8 \mathrm{~B})$ & 109.5 \\
\hline $\mathrm{O}(1)-\mathrm{C}(8)-\mathrm{H}(8 \mathrm{C})$ & 109.5 \\
\hline $\mathrm{H}(8 \mathrm{~A})-\mathrm{C}(8)-\mathrm{H}(8 \mathrm{C})$ & 109.5 \\
\hline $\mathrm{H}(8 \mathrm{~B})-\mathrm{C}(8)-\mathrm{H}(8 \mathrm{C})$ & 109.5 \\
\hline $\mathrm{C}(10)-\mathrm{C}(9)-\mathrm{C}(1)$ & $113.85(17)$ \\
\hline $\mathrm{C}(10)-\mathrm{C}(9)-\mathrm{H}(9 \mathrm{~A})$ & 108.8 \\
\hline $\mathrm{C}(1)-\mathrm{C}(9)-\mathrm{H}(9 \mathrm{~A})$ & 108.8 \\
\hline $\mathrm{C}(10)-\mathrm{C}(9)-\mathrm{H}(9 \mathrm{AB})$ & 108.8 \\
\hline $\mathrm{C}(1)-\mathrm{C}(9)-\mathrm{H}(9 \mathrm{AB})$ & 108.8 \\
\hline $\mathrm{H}(9 \mathrm{~A})-\mathrm{C}(9)-\mathrm{H}(9 \mathrm{AB})$ & 107.7 \\
\hline $\mathrm{O}(2)-\mathrm{C}(10)-\mathrm{C}(11)$ & $120.24(19)$ \\
\hline $\mathrm{O}(2)-\mathrm{C}(10)-\mathrm{C}(9)$ & $121.19(18)$ \\
\hline $\mathrm{C}(11)-\mathrm{C}(10)-\mathrm{C}(9)$ & $118.56(18)$ \\
\hline $\mathrm{C}(16)-\mathrm{C}(11)-\mathrm{C}(12)$ & 119.17(19) \\
\hline $\mathrm{C}(16)-\mathrm{C}(11)-\mathrm{C}(10)$ & $122.06(19)$ \\
\hline$C(12)-C(11)-C(10)$ & $118.76(19)$ \\
\hline $\mathrm{C}(11)-\mathrm{C}(12)-\mathrm{C}(13)$ & $120.4(2)$ \\
\hline $\mathrm{C}(11)-\mathrm{C}(12)-\mathrm{H}(12)$ & 119.8 \\
\hline $\mathrm{C}(13)-\mathrm{C}(12)-\mathrm{H}(12)$ & 119.8 \\
\hline $\mathrm{C}(14)-\mathrm{C}(13)-\mathrm{C}(12)$ & $120.1(2)$ \\
\hline $\mathrm{C}(14)-\mathrm{C}(13)-\mathrm{H}(13)$ & 119.9 \\
\hline $\mathrm{C}(12)-\mathrm{C}(13)-\mathrm{H}(13)$ & 119.9 \\
\hline$C(15)-C(14)-C(13)$ & $119.7(2)$ \\
\hline $\mathrm{C}(15)-\mathrm{C}(14)-\mathrm{H}(14)$ & 120.1 \\
\hline $\mathrm{C}(13)-\mathrm{C}(14)-\mathrm{H}(14)$ & 120.1 \\
\hline $\mathrm{C}(14)-\mathrm{C}(15)-\mathrm{C}(16)$ & $120.3(2)$ \\
\hline $\mathrm{C}(14)-\mathrm{C}(15)-\mathrm{H}(15)$ & 119.9 \\
\hline $\mathrm{C}(16)-\mathrm{C}(15)-\mathrm{H}(15)$ & 119.9 \\
\hline$C(11)-C(16)-C(15)$ & $120.3(2)$ \\
\hline $\mathrm{C}(11)-\mathrm{C}(16)-\mathrm{H}(16)$ & 119.9 \\
\hline $\mathrm{C}(15)-\mathrm{C}(16)-\mathrm{H}(16)$ & 119.9 \\
\hline $\mathrm{C}(18)-\mathrm{C}(17)-\mathrm{C}(1)$ & $113.83(17)$ \\
\hline C(18)-C(17)-H(17A) & 108.8 \\
\hline C(1)-C(17)-H(17A) & 108.8 \\
\hline
\end{tabular}




\begin{tabular}{ll}
$\mathrm{C}(18)-\mathrm{C}(17)-\mathrm{H}(17 \mathrm{~B})$ & 108.8 \\
$\mathrm{C}(1)-\mathrm{C}(17)-\mathrm{H}(17 \mathrm{~B})$ & 108.8 \\
$\mathrm{H}(17 \mathrm{~A})-\mathrm{C}(17)-\mathrm{H}(17 \mathrm{~B})$ & 107.7 \\
$\mathrm{O}(3)-\mathrm{C}(18)-\mathrm{C}(19)$ & $120.1(2)$ \\
$\mathrm{O}(3)-\mathrm{C}(18)-\mathrm{C}(17)$ & $120.8(2)$ \\
$\mathrm{C}(19)-\mathrm{C}(18)-\mathrm{C}(17)$ & $119.07(19)$ \\
$\mathrm{C}(24)-\mathrm{C}(19)-\mathrm{C}(20)$ & $119.0(2)$ \\
$\mathrm{C}(24)-\mathrm{C}(19)-\mathrm{C}(18)$ & $123.0(2)$ \\
$\mathrm{C}(20)-\mathrm{C}(19)-\mathrm{C}(18)$ & $118.0(2)$ \\
$\mathrm{C}(21)-\mathrm{C}(20)-\mathrm{C}(19)$ & $120.3(2)$ \\
$\mathrm{C}(21)-\mathrm{C}(20)-\mathrm{H}(20)$ & 119.9 \\
$\mathrm{C}(19)-\mathrm{C}(20)-\mathrm{H}(20)$ & 119.9 \\
$\mathrm{C}(20)-\mathrm{C}(21)-\mathrm{C}(22)$ & $120.4(2)$ \\
$\mathrm{C}(20)-\mathrm{C}(21)-\mathrm{H}(21)$ & 119.8 \\
$\mathrm{C}(22)-\mathrm{C}(21)-\mathrm{H}(21)$ & 119.8 \\
$\mathrm{C}(21)-\mathrm{C}(22)-\mathrm{C}(23)$ & $120.0(2)$ \\
$\mathrm{C}(21)-\mathrm{C}(22)-\mathrm{H}(22)$ & 120.0 \\
$\mathrm{C}(23)-\mathrm{C}(22)-\mathrm{H}(22)$ & 120.0 \\
$\mathrm{C}(24)-\mathrm{C}(23)-\mathrm{C}(22)$ & $119.6(3)$ \\
$\mathrm{C}(24)-\mathrm{C}(23)-\mathrm{H}(23)$ & 120.2 \\
$\mathrm{C}(22)-\mathrm{C}(23)-\mathrm{H}(23)$ & 120.2 \\
$\mathrm{C}(23)-\mathrm{C}(24)-\mathrm{C}(19)$ & $120.7(2)$ \\
$\mathrm{C}(23)-\mathrm{C}(24)-\mathrm{H}(24)$ & 119.6 \\
$\mathrm{C}(19)-\mathrm{C}(24)-\mathrm{H}(24)$ & 119.6 \\
& \\
\hline & \\
& \\
& \\
& \\
&
\end{tabular}

Symmetry transformations used to generate equivalent atoms: 
Table S4. Anisotropic displacement parameters $\left(\AA^{2} \times 10^{3}\right)$ for i17948. The anisotropic displacement factor exponent takes the form: $-2 \pi^{2}\left[h^{2} a^{* 2} U^{11}+\ldots+2 h k^{*} b^{*} U^{12}\right]$

\begin{tabular}{|c|c|c|c|c|c|c|}
\hline & $\mathrm{U}^{11}$ & $\mathrm{U}^{22}$ & $\mathrm{U}^{33}$ & $\mathrm{U}^{23}$ & $\mathrm{U}^{13}$ & $\mathrm{U}^{12}$ \\
\hline $\mathrm{O}(1)$ & 21(1) & $23(1)$ & $31(1)$ & $-3(1)$ & $6(1)$ & $-5(1)$ \\
\hline $\mathrm{O}(2)$ & $17(1)$ & $24(1)$ & $28(1)$ & $0(1)$ & $-2(1)$ & $-4(1)$ \\
\hline $\mathrm{O}(3)$ & $34(1)$ & $27(1)$ & $28(1)$ & $1(1)$ & $10(1)$ & $9(1)$ \\
\hline $\mathrm{C}(1)$ & $16(1)$ & $20(1)$ & $20(1)$ & $-1(1)$ & $2(1)$ & $-1(1)$ \\
\hline $\mathrm{C}(2)$ & $18(1)$ & $18(1)$ & $20(1)$ & $-1(1)$ & $0(1)$ & 1(1) \\
\hline $\mathrm{C}(3)$ & $18(1)$ & $19(1)$ & $24(1)$ & $0(1)$ & $1(1)$ & $0(1)$ \\
\hline $\mathrm{C}(4)$ & $22(1)$ & $24(1)$ & $29(1)$ & $-5(1)$ & $-1(1)$ & $-1(1)$ \\
\hline$C(5)$ & $32(1)$ & $26(1)$ & 21(1) & $-5(1)$ & $-1(1)$ & $-1(1)$ \\
\hline$C(6)$ & $35(1)$ & $29(1)$ & $22(1)$ & $-3(1)$ & $7(1)$ & $-3(1)$ \\
\hline$C(7)$ & $29(1)$ & $22(1)$ & $22(1)$ & $-2(1)$ & $5(1)$ & $-4(1)$ \\
\hline$C(8)$ & $23(1)$ & 21(1) & $43(2)$ & $-2(1)$ & $4(1)$ & $-4(1)$ \\
\hline $\mathrm{C}(9)$ & $16(1)$ & 19(1) & $20(1)$ & $0(1)$ & $1(1)$ & $-2(1)$ \\
\hline$C(10)$ & $16(1)$ & $20(1)$ & $18(1)$ & $-3(1)$ & $3(1)$ & $-2(1)$ \\
\hline $\mathrm{C}(11)$ & $16(1)$ & 19(1) & $18(1)$ & $0(1)$ & $0(1)$ & $0(1)$ \\
\hline$C(12)$ & $23(1)$ & $23(1)$ & $42(1)$ & 2(1) & $-13(1)$ & $-3(1)$ \\
\hline$C(13)$ & $33(1)$ & $25(1)$ & $51(2)$ & $10(1)$ & $-16(1)$ & $-2(1)$ \\
\hline$C(14)$ & $26(1)$ & $20(1)$ & $31(1)$ & $3(1)$ & $-3(1)$ & $-2(1)$ \\
\hline$C(15)$ & $32(1)$ & $23(1)$ & $35(1)$ & $5(1)$ & $-11(1)$ & $-9(1)$ \\
\hline$C(16)$ & $26(1)$ & 21(1) & $32(1)$ & $5(1)$ & $-10(1)$ & $-5(1)$ \\
\hline $\mathrm{C}(17)$ & $22(1)$ & $20(1)$ & $20(1)$ & $0(1)$ & $3(1)$ & $1(1)$ \\
\hline$C(18)$ & $24(1)$ & $16(1)$ & 21(1) & $1(1)$ & $6(1)$ & $-3(1)$ \\
\hline $\mathrm{C}(19)$ & $27(1)$ & 21(1) & $19(1)$ & $-2(1)$ & $3(1)$ & $-5(1)$ \\
\hline$C(20)$ & $34(1)$ & $30(1)$ & $20(1)$ & $-2(1)$ & $3(1)$ & $-11(1)$ \\
\hline $\mathrm{C}(21)$ & $45(2)$ & $47(2)$ & 21(1) & $2(1)$ & $-3(1)$ & $-19(1)$ \\
\hline$C(22)$ & $43(2)$ & $39(1)$ & $28(1)$ & $8(1)$ & $-12(1)$ & $-16(1)$ \\
\hline$C(23)$ & $35(1)$ & $30(1)$ & $34(2)$ & $3(1)$ & $-11(1)$ & $-6(1)$ \\
\hline $\mathrm{C}(24)$ & $29(1)$ & $22(1)$ & $23(1)$ & $1(1)$ & $-5(1)$ & $-4(1)$ \\
\hline
\end{tabular}




\section{Crystal data and structure refinement for compound $\mathbf{3 q}$}

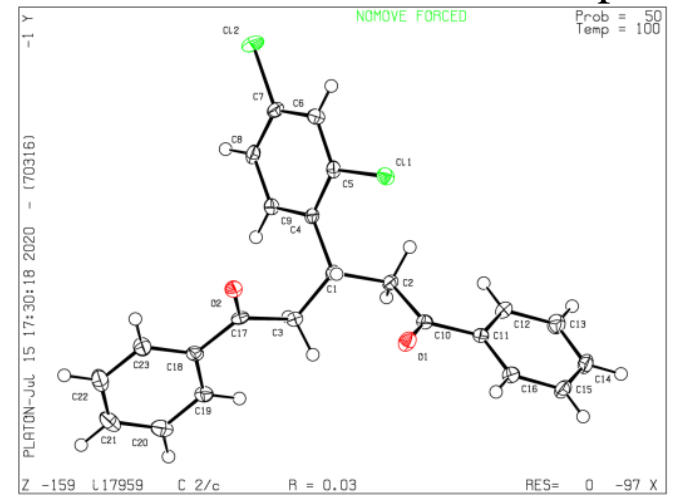

Figure S2. Single X-ray crystal structure of 1, 5-dicarbonyls 3q (the thermal ellipsoid was drawn at the $50 \%$ probability level)

Table S5. Crystal data and structure refinement for i17959.

CCDC

Identification code

Empirical formula

Formula weight

Temperature

Wavelength

Crystal system

Space group

Unit cell dimensions

Z, Volume,

Density (calculated)

Absorption coefficient

$\mathrm{F}(000)$

Crystal size

Theta range for data collection

Index ranges

Reflections collected

Independent reflections

Completeness to theta $=65.204^{\circ}$

Absorption correction

Max. and min. transmission

Refinement method

Data / restraints / parameters

Goodness-of-fit on $\mathrm{F}^{2}$

Final $\mathrm{R}$ indices [I>2sigma(I)]
2031234

i17959

C23 H18 Cl2 O2

397.27

100.0(2) K

$1.54178 \AA$

Monoclinic

$\mathrm{C} 2 / \mathrm{c}$

$\mathrm{a}=22.7208(6) \AA$

$\alpha=90^{\circ}$.

$\mathrm{b}=4.89590(10) \AA$

$\beta=91.140(2)^{\circ}$.

$\mathrm{c}=34.0185(10) \AA$

$\gamma=90^{\circ}$.

8, 3783.43(17) $\AA^{3}$

$1.395 \mathrm{Mg} / \mathrm{m}^{3}$

$3.207 \mathrm{~mm}^{-1}$

1648

$0.194 \times 0.081 \times 0.043 \mathrm{~mm}^{3}$

3.892 to $65.204^{\circ}$.

$-26<=\mathrm{h}<=26,-5<=\mathrm{k}<=5,-40<=1<=40$

38496

$3231[\mathrm{R}(\mathrm{int})=0.0713]$

$99.9 \%$

Numerical

1 and 0.8202

Full-matrix least-squares on $\mathrm{F}^{2}$

$3231 / 0 / 244$

1.049

$\mathrm{R} 1=0.0302, \mathrm{wR} 2=0.0724$ 
$\mathrm{R}$ indices (all data)

Extinction coefficient

Largest diff. peak and hole

$$
\begin{aligned}
& \mathrm{R} 1=0.0383, \mathrm{wR} 2=0.0770 \\
& \mathrm{n} / \mathrm{a} \\
& 0.241 \text { and }-0.284 \mathrm{e} . \AA^{-3}
\end{aligned}
$$

\begin{tabular}{|c|c|c|c|c|}
\hline & $\mathrm{x}$ & $\mathrm{y}$ & $\mathrm{z}$ & $\mathrm{U}(\mathrm{eq})$ \\
\hline $\mathrm{Cl}(1)$ & $4795(1)$ & $8545(1)$ & $4502(1)$ & $19(1)$ \\
\hline $\mathrm{Cl}(2)$ & $6684(1)$ & $3518(1)$ & $5133(1)$ & $33(1)$ \\
\hline $\mathrm{O}(1)$ & 3913(1) & 7291(3) & $3466(1)$ & $22(1)$ \\
\hline $\mathrm{O}(2)$ & $5856(1)$ & $7442(2)$ & $3366(1)$ & 19(1) \\
\hline $\mathrm{C}(1)$ & $4944(1)$ & $5024(3)$ & $3775(1)$ & $15(1)$ \\
\hline$C(2)$ & $4339(1)$ & $3838(3)$ & $3877(1)$ & $15(1)$ \\
\hline $\mathrm{C}(3)$ & $5143(1)$ & $3881(3)$ & $3382(1)$ & $16(1)$ \\
\hline $\mathrm{C}(4)$ & $5387(1)$ & $4587(3)$ & $4110(1)$ & $14(1)$ \\
\hline$C(5)$ & $5355(1)$ & $6130(3)$ & $4454(1)$ & $15(1)$ \\
\hline$C(6)$ & $5746(1)$ & $5844(3)$ & $4769(1)$ & 19(1) \\
\hline$C(7)$ & $6187(1)$ & $3910(4)$ & $4741(1)$ & $19(1)$ \\
\hline$C(8)$ & $6237(1)$ & $2303(4)$ & $4411(1)$ & 19(1) \\
\hline $\mathrm{C}(9)$ & $5838(1)$ & $2657(3)$ & $4100(1)$ & $16(1)$ \\
\hline$C(10)$ & $3829(1)$ & $5317(3)$ & $3677(1)$ & $16(1)$ \\
\hline $\mathrm{C}(11)$ & $3220(1)$ & $4303(3)$ & $3748(1)$ & $16(1)$ \\
\hline$C(12)$ & $3105(1)$ & $2213(4)$ & $4014(1)$ & $19(1)$ \\
\hline$C(13)$ & $2531(1)$ & $1346(4)$ & $4070(1)$ & $23(1)$ \\
\hline$C(14)$ & 2072(1) & $2520(4)$ & $3858(1)$ & $22(1)$ \\
\hline$C(15)$ & 2181(1) & $4605(4)$ & $3593(1)$ & $21(1)$ \\
\hline$C(16)$ & $2752(1)$ & $5513(4)$ & $3541(1)$ & 19(1) \\
\hline$C(17)$ & $5683(1)$ & $5281(3)$ & $3225(1)$ & $16(1)$ \\
\hline $\mathrm{C}(18)$ & $5996(1)$ & $4018(3)$ & $2889(1)$ & $17(1)$ \\
\hline$C(19)$ & $5750(1)$ & $1855(4)$ & $2674(1)$ & $20(1)$ \\
\hline$C(20)$ & $6036(1)$ & $795(4)$ & $2352(1)$ & $24(1)$ \\
\hline$C(21)$ & $6574(1)$ & $1849(4)$ & $2246(1)$ & $26(1)$ \\
\hline$C(22)$ & $6830(1)$ & $3956(4)$ & $2462(1)$ & $26(1)$ \\
\hline$C(23)$ & $6542(1)$ & $5054(4)$ & $2780(1)$ & $21(1)$ \\
\hline
\end{tabular}

Table S6. Atomic coordinates ( $\left.x \mathbf{1 0}^{4}\right)$ and equivalent isotropic displacement parameters $\left(\AA^{2} \times 1^{3}\right)$ for i17959. $U(e q)$ is defined as one third of the trace of the orthogonalized $U^{i j}$ tensor. 
Table S7. Bond lengths $[\AA ̊]$ and angles $\left[{ }^{\circ}\right]$ for $\mathbf{i} 17959$.

\begin{tabular}{|c|c|}
\hline $\mathrm{Cl}(1)-\mathrm{C}(5)$ & $1.7457(16)$ \\
\hline $\mathrm{Cl}(2)-\mathrm{C}(7)$ & $1.7421(16)$ \\
\hline $\mathrm{O}(1)-\mathrm{C}(10)$ & $1.221(2)$ \\
\hline $\mathrm{O}(2)-\mathrm{C}(17)$ & $1.223(2)$ \\
\hline $\mathrm{C}(1)-\mathrm{C}(4)$ & $1.520(2)$ \\
\hline $\mathrm{C}(1)-\mathrm{C}(3)$ & $1.529(2)$ \\
\hline $\mathrm{C}(1)-\mathrm{C}(2)$ & $1.537(2)$ \\
\hline $\mathrm{C}(1)-\mathrm{H}(1)$ & 1.0000 \\
\hline $\mathrm{C}(2)-\mathrm{C}(10)$ & $1.516(2)$ \\
\hline $\mathrm{C}(2)-\mathrm{H}(2 \mathrm{~A})$ & 0.9900 \\
\hline $\mathrm{C}(2)-\mathrm{H}(2 \mathrm{AB})$ & 0.9900 \\
\hline $\mathrm{C}(3)-\mathrm{C}(17)$ & $1.513(2)$ \\
\hline $\mathrm{C}(3)-\mathrm{H}(3 \mathrm{~A})$ & 0.9900 \\
\hline $\mathrm{C}(3)-\mathrm{H}(3 \mathrm{AB})$ & 0.9900 \\
\hline $\mathrm{C}(4)-\mathrm{C}(9)$ & $1.396(2)$ \\
\hline $\mathrm{C}(4)-\mathrm{C}(5)$ & $1.397(2)$ \\
\hline$C(5)-C(6)$ & $1.386(2)$ \\
\hline$C(6)-C(7)$ & $1.382(2)$ \\
\hline $\mathrm{C}(6)-\mathrm{H}(6)$ & 0.9500 \\
\hline$C(7)-C(8)$ & $1.376(2)$ \\
\hline $\mathrm{C}(8)-\mathrm{C}(9)$ & $1.390(2)$ \\
\hline $\mathrm{C}(8)-\mathrm{H}(8)$ & 0.9500 \\
\hline $\mathrm{C}(9)-\mathrm{H}(9)$ & 0.9500 \\
\hline $\mathrm{C}(10)-\mathrm{C}(11)$ & $1.495(2)$ \\
\hline $\mathrm{C}(11)-\mathrm{C}(12)$ & $1.393(2)$ \\
\hline C(11)-C(16) & $1.397(2)$ \\
\hline $\mathrm{C}(12)-\mathrm{C}(13)$ & $1.389(2)$ \\
\hline $\mathrm{C}(12)-\mathrm{H}(12)$ & 0.9500 \\
\hline C(13)-C(14) & $1.383(2)$ \\
\hline $\mathrm{C}(13)-\mathrm{H}(13)$ & 0.9500 \\
\hline C(14)-C(15) & $1.385(3)$ \\
\hline $\mathrm{C}(14)-\mathrm{H}(14)$ & 0.9500 \\
\hline$C(15)-C(16)$ & $1.386(2)$ \\
\hline $\mathrm{C}(15)-\mathrm{H}(15)$ & 0.9500 \\
\hline $\mathrm{C}(16)-\mathrm{H}(16)$ & 0.9500 \\
\hline $\mathrm{C}(17)-\mathrm{C}(18)$ & $1.491(2)$ \\
\hline
\end{tabular}




\begin{tabular}{|c|c|}
\hline C(18)-C(19) & $1.396(2)$ \\
\hline $\mathrm{C}(18)-\mathrm{C}(23)$ & $1.398(2)$ \\
\hline $\mathrm{C}(19)-\mathrm{C}(20)$ & $1.387(2)$ \\
\hline C(19)-H(19) & 0.9500 \\
\hline$C(20)-C(21)$ & $1.382(3)$ \\
\hline C(20)-H(20) & 0.9500 \\
\hline $\mathrm{C}(21)-\mathrm{C}(22)$ & $1.388(3)$ \\
\hline $\mathrm{C}(21)-\mathrm{H}(21)$ & 0.9500 \\
\hline $\mathrm{C}(22)-\mathrm{C}(23)$ & $1.384(2)$ \\
\hline $\mathrm{C}(22)-\mathrm{H}(22)$ & 0.9500 \\
\hline $\mathrm{C}(23)-\mathrm{H}(23)$ & 0.9500 \\
\hline$C(4)-C(1)-C(3)$ & $113.67(13)$ \\
\hline $\mathrm{C}(4)-\mathrm{C}(1)-\mathrm{C}(2)$ & $111.08(12)$ \\
\hline$C(3)-C(1)-C(2)$ & 109.91(13) \\
\hline $\mathrm{C}(4)-\mathrm{C}(1)-\mathrm{H}(1)$ & 107.3 \\
\hline $\mathrm{C}(3)-\mathrm{C}(1)-\mathrm{H}(1)$ & 107.3 \\
\hline $\mathrm{C}(2)-\mathrm{C}(1)-\mathrm{H}(1)$ & 107.3 \\
\hline$C(10)-C(2)-C(1)$ & $113.36(13)$ \\
\hline $\mathrm{C}(10)-\mathrm{C}(2)-\mathrm{H}(2 \mathrm{~A})$ & 108.9 \\
\hline $\mathrm{C}(1)-\mathrm{C}(2)-\mathrm{H}(2 \mathrm{~A})$ & 108.9 \\
\hline $\mathrm{C}(10)-\mathrm{C}(2)-\mathrm{H}(2 \mathrm{AB})$ & 108.9 \\
\hline $\mathrm{C}(1)-\mathrm{C}(2)-\mathrm{H}(2 \mathrm{AB})$ & 108.9 \\
\hline $\mathrm{H}(2 \mathrm{~A})-\mathrm{C}(2)-\mathrm{H}(2 \mathrm{AB})$ & 107.7 \\
\hline $\mathrm{C}(17)-\mathrm{C}(3)-\mathrm{C}(1)$ & $113.58(13)$ \\
\hline $\mathrm{C}(17)-\mathrm{C}(3)-\mathrm{H}(3 \mathrm{~A})$ & 108.9 \\
\hline $\mathrm{C}(1)-\mathrm{C}(3)-\mathrm{H}(3 \mathrm{~A})$ & 108.9 \\
\hline $\mathrm{C}(17)-\mathrm{C}(3)-\mathrm{H}(3 \mathrm{AB})$ & 108.9 \\
\hline $\mathrm{C}(1)-\mathrm{C}(3)-\mathrm{H}(3 \mathrm{AB})$ & 108.9 \\
\hline $\mathrm{H}(3 \mathrm{~A})-\mathrm{C}(3)-\mathrm{H}(3 \mathrm{AB})$ & 107.7 \\
\hline$C(9)-C(4)-C(5)$ & $115.92(14)$ \\
\hline $\mathrm{C}(9)-\mathrm{C}(4)-\mathrm{C}(1)$ & $123.62(14)$ \\
\hline$C(5)-C(4)-C(1)$ & $120.46(14)$ \\
\hline$C(6)-C(5)-C(4)$ & $123.35(15)$ \\
\hline $\mathrm{C}(6)-\mathrm{C}(5)-\mathrm{Cl}(1)$ & $116.94(12)$ \\
\hline $\mathrm{C}(4)-\mathrm{C}(5)-\mathrm{Cl}(1)$ & $119.70(12)$ \\
\hline$C(7)-C(6)-C(5)$ & $118.07(15)$ \\
\hline $\mathrm{C}(7)-\mathrm{C}(6)-\mathrm{H}(6)$ & 121.0 \\
\hline $\mathrm{C}(5)-\mathrm{C}(6)-\mathrm{H}(6)$ & 121.0 \\
\hline
\end{tabular}




\begin{tabular}{|c|c|}
\hline $\mathrm{C}(8)-\mathrm{C}(7)-\mathrm{C}(6)$ & $121.30(15)$ \\
\hline $\mathrm{C}(8)-\mathrm{C}(7)-\mathrm{Cl}(2)$ & $119.88(13)$ \\
\hline $\mathrm{C}(6)-\mathrm{C}(7)-\mathrm{Cl}(2)$ & $118.82(13)$ \\
\hline$C(7)-C(8)-C(9)$ & $119.10(15)$ \\
\hline $\mathrm{C}(7)-\mathrm{C}(8)-\mathrm{H}(8)$ & 120.4 \\
\hline $\mathrm{C}(9)-\mathrm{C}(8)-\mathrm{H}(8)$ & 120.4 \\
\hline $\mathrm{C}(8)-\mathrm{C}(9)-\mathrm{C}(4)$ & $122.25(15)$ \\
\hline $\mathrm{C}(8)-\mathrm{C}(9)-\mathrm{H}(9)$ & 118.9 \\
\hline $\mathrm{C}(4)-\mathrm{C}(9)-\mathrm{H}(9)$ & 118.9 \\
\hline $\mathrm{O}(1)-\mathrm{C}(10)-\mathrm{C}(11)$ & $120.87(15)$ \\
\hline $\mathrm{O}(1)-\mathrm{C}(10)-\mathrm{C}(2)$ & $121.07(14)$ \\
\hline $\mathrm{C}(11)-\mathrm{C}(10)-\mathrm{C}(2)$ & $118.06(14)$ \\
\hline$C(12)-C(11)-C(16)$ & $119.21(15)$ \\
\hline$C(12)-C(11)-C(10)$ & $122.39(15)$ \\
\hline$C(16)-C(11)-C(10)$ & $118.41(15)$ \\
\hline $\mathrm{C}(13)-\mathrm{C}(12)-\mathrm{C}(11)$ & $120.23(16)$ \\
\hline $\mathrm{C}(13)-\mathrm{C}(12)-\mathrm{H}(12)$ & 119.9 \\
\hline $\mathrm{C}(11)-\mathrm{C}(12)-\mathrm{H}(12)$ & 119.9 \\
\hline$C(14)-C(13)-C(12)$ & $120.09(16)$ \\
\hline $\mathrm{C}(14)-\mathrm{C}(13)-\mathrm{H}(13)$ & 120.0 \\
\hline $\mathrm{C}(12)-\mathrm{C}(13)-\mathrm{H}(13)$ & 120.0 \\
\hline$C(13)-C(14)-C(15)$ & $120.14(16)$ \\
\hline $\mathrm{C}(13)-\mathrm{C}(14)-\mathrm{H}(14)$ & 119.9 \\
\hline $\mathrm{C}(15)-\mathrm{C}(14)-\mathrm{H}(14)$ & 119.9 \\
\hline$C(14)-C(15)-C(16)$ & $120.08(16)$ \\
\hline $\mathrm{C}(14)-\mathrm{C}(15)-\mathrm{H}(15)$ & 120.0 \\
\hline $\mathrm{C}(16)-\mathrm{C}(15)-\mathrm{H}(15)$ & 120.0 \\
\hline$C(15)-C(16)-C(11)$ & $120.24(16)$ \\
\hline $\mathrm{C}(15)-\mathrm{C}(16)-\mathrm{H}(16)$ & 119.9 \\
\hline $\mathrm{C}(11)-\mathrm{C}(16)-\mathrm{H}(16)$ & 119.9 \\
\hline $\mathrm{O}(2)-\mathrm{C}(17)-\mathrm{C}(18)$ & $120.47(14)$ \\
\hline $\mathrm{O}(2)-\mathrm{C}(17)-\mathrm{C}(3)$ & $120.53(14)$ \\
\hline$C(18)-C(17)-C(3)$ & $118.99(14)$ \\
\hline C(19)-C(18)-C(23) & $119.04(15)$ \\
\hline$C(19)-C(18)-C(17)$ & $121.58(15)$ \\
\hline $\mathrm{C}(23)-\mathrm{C}(18)-\mathrm{C}(17)$ & $119.36(15)$ \\
\hline $\mathrm{C}(20)-\mathrm{C}(19)-\mathrm{C}(18)$ & $120.58(16)$ \\
\hline C(20)-C(19)-H(19) & 119.7 \\
\hline
\end{tabular}




$\begin{array}{ll}\mathrm{C}(18)-\mathrm{C}(19)-\mathrm{H}(19) & 119.7 \\ \mathrm{C}(21)-\mathrm{C}(20)-\mathrm{C}(19) & 119.77(17) \\ \mathrm{C}(21)-\mathrm{C}(20)-\mathrm{H}(20) & 120.1 \\ \mathrm{C}(19)-\mathrm{C}(20)-\mathrm{H}(20) & 120.1 \\ \mathrm{C}(20)-\mathrm{C}(21)-\mathrm{C}(22) & 120.28(16) \\ \mathrm{C}(20)-\mathrm{C}(21)-\mathrm{H}(21) & 119.9 \\ \mathrm{C}(22)-\mathrm{C}(21)-\mathrm{H}(21) & 119.9 \\ \mathrm{C}(23)-\mathrm{C}(22)-\mathrm{C}(21) & 120.19(17) \\ \mathrm{C}(23)-\mathrm{C}(22)-\mathrm{H}(22) & 119.9 \\ \mathrm{C}(21)-\mathrm{C}(22)-\mathrm{H}(22) & 119.9 \\ \mathrm{C}(22)-\mathrm{C}(23)-\mathrm{C}(18) & 120.11(17) \\ \mathrm{C}(22)-\mathrm{C}(23)-\mathrm{H}(23) & 119.9 \\ \mathrm{C}(18)-\mathrm{C}(23)-\mathrm{H}(23) & 119.9\end{array}$

Symmetry transformations used to generate equivalent atoms: 
Table S8. Anisotropic displacement parameters $\left(\AA^{2} \times 10^{3}\right)$ for i17959. The anisotropic displacement factor exponent takes the form: $-2 \pi^{2}\left[h^{2} a^{* 2} U^{11}+\ldots+2 h k^{*} b^{*} U^{12}\right]$

\begin{tabular}{|c|c|c|c|c|c|c|}
\hline & $\mathrm{U}^{11}$ & $\mathrm{U}^{22}$ & $\mathrm{U}^{33}$ & $\mathrm{U}^{23}$ & $\mathrm{U}^{13}$ & $\mathrm{U}^{12}$ \\
\hline $\mathrm{Cl}(1)$ & $20(1)$ & $20(1)$ & $17(1)$ & $0(1)$ & 1(1) & $5(1)$ \\
\hline $\mathrm{Cl}(2)$ & $23(1)$ & $52(1)$ & $22(1)$ & $1(1)$ & $-11(1)$ & $7(1)$ \\
\hline $\mathrm{O}(1)$ & $17(1)$ & $25(1)$ & $24(1)$ & $9(1)$ & $-2(1)$ & $-2(1)$ \\
\hline $\mathrm{O}(2)$ & $19(1)$ & $21(1)$ & $18(1)$ & $-2(1)$ & $0(1)$ & $-2(1)$ \\
\hline $\mathrm{C}(1)$ & $12(1)$ & $17(1)$ & $15(1)$ & $1(1)$ & $-1(1)$ & $-1(1)$ \\
\hline $\mathrm{C}(2)$ & $13(1)$ & $18(1)$ & $15(1)$ & $1(1)$ & $-1(1)$ & $-1(1)$ \\
\hline C(3) & $15(1)$ & $20(1)$ & $14(1)$ & $0(1)$ & $-2(1)$ & $0(1)$ \\
\hline $\mathrm{C}(4)$ & $12(1)$ & $16(1)$ & $14(1)$ & $2(1)$ & $1(1)$ & $-4(1)$ \\
\hline$C(5)$ & $13(1)$ & $16(1)$ & $17(1)$ & $2(1)$ & $3(1)$ & 1(1) \\
\hline$C(6)$ & $20(1)$ & $22(1)$ & $14(1)$ & $-1(1)$ & $0(1)$ & $-2(1)$ \\
\hline $\mathrm{C}(7)$ & $14(1)$ & $28(1)$ & $16(1)$ & $4(1)$ & $-3(1)$ & $-2(1)$ \\
\hline $\mathrm{C}(8)$ & $11(1)$ & $22(1)$ & $23(1)$ & $4(1)$ & $1(1)$ & 2(1) \\
\hline C(9) & $14(1)$ & $18(1)$ & $16(1)$ & $0(1)$ & $2(1)$ & $-2(1)$ \\
\hline$C(10)$ & $16(1)$ & $18(1)$ & $14(1)$ & $-2(1)$ & $-1(1)$ & 1(1) \\
\hline $\mathrm{C}(11)$ & $15(1)$ & $19(1)$ & $13(1)$ & $-4(1)$ & $-1(1)$ & $0(1)$ \\
\hline$C(12)$ & $15(1)$ & $22(1)$ & $19(1)$ & $0(1)$ & $-3(1)$ & $0(1)$ \\
\hline $\mathrm{C}(13)$ & $20(1)$ & $24(1)$ & $24(1)$ & $1(1)$ & $1(1)$ & $-3(1)$ \\
\hline$C(14)$ & $13(1)$ & $26(1)$ & $26(1)$ & $-5(1)$ & $1(1)$ & $-3(1)$ \\
\hline$C(15)$ & $14(1)$ & $25(1)$ & $24(1)$ & $-3(1)$ & $-3(1)$ & $3(1)$ \\
\hline$C(16)$ & $18(1)$ & $22(1)$ & $17(1)$ & $-2(1)$ & $-2(1)$ & 1(1) \\
\hline $\mathrm{C}(17)$ & $16(1)$ & $19(1)$ & $13(1)$ & $3(1)$ & $-5(1)$ & $2(1)$ \\
\hline$C(18)$ & $18(1)$ & $20(1)$ & $13(1)$ & $3(1)$ & $-2(1)$ & $4(1)$ \\
\hline $\mathrm{C}(19)$ & $20(1)$ & $23(1)$ & $16(1)$ & $1(1)$ & $-2(1)$ & 1(1) \\
\hline$C(20)$ & $31(1)$ & $24(1)$ & $17(1)$ & $-1(1)$ & $-3(1)$ & $5(1)$ \\
\hline $\mathrm{C}(21)$ & $34(1)$ & $26(1)$ & $17(1)$ & $2(1)$ & $6(1)$ & 11(1) \\
\hline$C(22)$ & $23(1)$ & $30(1)$ & $25(1)$ & $2(1)$ & $8(1)$ & $3(1)$ \\
\hline$C(23)$ & $20(1)$ & $24(1)$ & $20(1)$ & $1(1)$ & $2(1)$ & $0(1)$ \\
\hline
\end{tabular}




\section{Crystal data and structure refinement for compound $\mathbf{3 t}$}

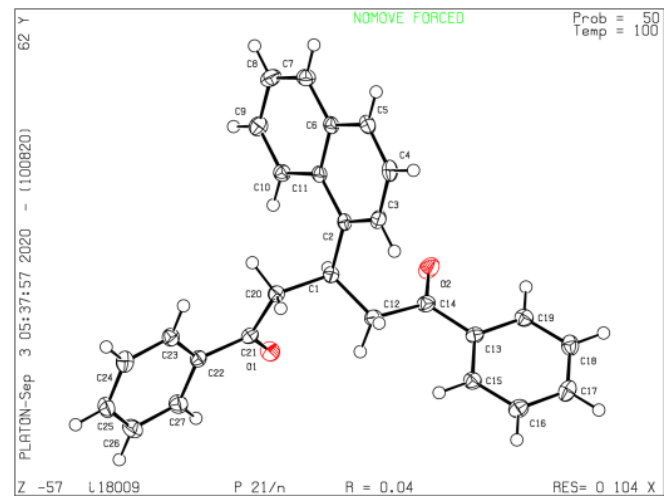

Figure S3. Single X-ray crystal structure of 1, 5-dicarbonyls $\mathbf{3 t}$ (the thermal ellipsoid was drawn at the 50\% probability level)

Table S9. Crystal data and structure refinement for i18009.

CCDC

Identification code

Empirical formula

Formula weight

Temperature

Wavelength

Crystal system

Space group

Unit cell dimensions

Z, Volume

Density (calculated)

Absorption coefficient

$\mathrm{F}(000)$

Crystal size

Theta range for data collection

Index ranges

Reflections collected

Independent reflections

Completeness to theta $=25.242^{\circ}$

Absorption correction

Max. and min. transmission

Refinement method

Data / restraints / parameters

Goodness-of-fit on $\mathrm{F}^{2}$

Final R indices [I>2sigma(I)]
2031246

i18009

$\mathrm{C} 27 \mathrm{H} 22 \mathrm{O} 2$

378.44

100.0(2) K

$0.71073 \AA$

Monoclinic

P 21/n

$\mathrm{a}=13.3568(5) \AA$

$\alpha=90^{\circ}$.

$\mathrm{b}=10.1409(3) \AA$

$\beta=96.328(2)^{\circ}$.

$\mathrm{c}=14.6406(5) \AA$ $\gamma=90^{\circ}$.

4, 1970.99(12) $\AA^{3}$

$1.275 \mathrm{Mg} / \mathrm{m}^{3}$

$0.079 \mathrm{~mm}^{-1}$

800

$0.150 \times 0.125 \times 0.125 \mathrm{~mm}^{3}$

1.959 to $27.105^{\circ}$.

$-17<=\mathrm{h}<=17,-12<=\mathrm{k}<=12,-18<=\mathrm{l}<=18$

83535

$4341[\mathrm{R}(\mathrm{int})=0.0721]$

$99.8 \%$

Numerical

0.9988 and 0.9689

Full-matrix least-squares on $\mathrm{F}^{2}$

4341 / 0 / 262

1.058

$\mathrm{R} 1=0.0384, \mathrm{wR} 2=0.0865$

\section{S-16}



$\mathrm{R}$ indices (all data)
$\mathrm{R} 1=0.0530, \mathrm{wR} 2=0.0949$
Extinction coefficient
$\mathrm{n} / \mathrm{a}$
Largest diff. peak and hole
0.267 and -0.219 e. $\AA^{-3}$

Table S10. Atomic coordinates $\left(x 1^{4}\right)$ and equivalent isotropic displacement parameters $\left(\AA^{2} \times 1^{3}\right)$ for i18009. $U(e q)$ is defined as one third of the trace of the orthogonalized $U^{i j}$ tensor.

\begin{tabular}{|c|c|c|c|c|}
\hline & $\mathrm{x}$ & $\mathrm{y}$ & $\mathrm{z}$ & $\mathrm{U}(\mathrm{eq})$ \\
\hline $\mathrm{O}(1)$ & $4534(1)$ & 7404(1) & $3042(1)$ & $23(1)$ \\
\hline $\mathrm{O}(2)$ & $5892(1)$ & $11210(1)$ & $2168(1)$ & $32(1)$ \\
\hline $\mathrm{C}(1)$ & $6331(1)$ & $8902(1)$ & $3152(1)$ & $18(1)$ \\
\hline $\mathrm{C}(2)$ & $7285(1)$ & $9578(1)$ & $3585(1)$ & $17(1)$ \\
\hline$C(3)$ & $8126(1)$ & $9700(1)$ & $3130(1)$ & $20(1)$ \\
\hline $\mathrm{C}(4)$ & $9017(1)$ & 10303(1) & $3541(1)$ & $22(1)$ \\
\hline$C(5)$ & $9067(1)$ & 10784(1) & $4415(1)$ & $20(1)$ \\
\hline$C(6)$ & $8233(1)$ & $10655(1)$ & $4927(1)$ & $18(1)$ \\
\hline$C(7)$ & $8287(1)$ & 11108(1) & $5846(1)$ & $22(1)$ \\
\hline $\mathrm{C}(8)$ & $7485(1)$ & 10973(1) & $6344(1)$ & $25(1)$ \\
\hline $\mathrm{C}(9)$ & $6594(1)$ & 10364(1) & $5947(1)$ & $23(1)$ \\
\hline $\mathrm{C}(10)$ & $6519(1)$ & $9915(1)$ & $5059(1)$ & $19(1)$ \\
\hline $\mathrm{C}(11)$ & 7331(1) & $10047(1)$ & $4513(1)$ & $16(1)$ \\
\hline $\mathrm{C}(12)$ & $6224(1)$ & $8904(1)$ & 2099(1) & $20(1)$ \\
\hline$C(13)$ & $6228(1)$ & 10486(1) & $703(1)$ & $18(1)$ \\
\hline $\mathrm{C}(14)$ & $6102(1)$ & $10283(1)$ & $1698(1)$ & $20(1)$ \\
\hline $\mathrm{C}(15)$ & $6334(1)$ & $9435(1)$ & $103(1)$ & $20(1)$ \\
\hline$C(16)$ & $6440(1)$ & $9677(1)$ & $-815(1)$ & $24(1)$ \\
\hline $\mathrm{C}(17)$ & $6453(1)$ & 10961(1) & $-1138(1)$ & $25(1)$ \\
\hline $\mathrm{C}(18)$ & $6357(1)$ & $12012(1)$ & $-545(1)$ & $23(1)$ \\
\hline $\mathrm{C}(19)$ & $6238(1)$ & $11774(1)$ & $368(1)$ & $20(1)$ \\
\hline$C(20)$ & $6304(1)$ & $7482(1)$ & $3526(1)$ & $19(1)$ \\
\hline $\mathrm{C}(21)$ & $5281(1)$ & $6824(1)$ & 3392(1) & $18(1)$ \\
\hline $\mathrm{C}(22)$ & $5217(1)$ & $5412(1)$ & $3683(1)$ & $18(1)$ \\
\hline $\mathrm{C}(23)$ & $6022(1)$ & $4771(1)$ & $4189(1)$ & $20(1)$ \\
\hline $\mathrm{C}(24)$ & $5956(1)$ & $3439(1)$ & $4395(1)$ & $24(1)$ \\
\hline $\mathrm{C}(25)$ & $5093(1)$ & $2743(1)$ & 4098(1) & $25(1)$ \\
\hline$C(26)$ & $4278(1)$ & $3375(1)$ & $3614(1)$ & $28(1)$ \\
\hline $\mathrm{C}(27)$ & $4337(1)$ & $4707(1)$ & $3411(1)$ & $24(1)$ \\
\hline
\end{tabular}


Table S11. Bond lengths $[\AA ̊ \AA]$ and angles $\left[{ }^{\circ}\right]$ for $\mathbf{i 1 8 0 0 9}$.

\begin{tabular}{|c|c|}
\hline $\mathrm{O}(1)-\mathrm{C}(21)$ & $1.2206(15)$ \\
\hline $\mathrm{O}(2)-\mathrm{C}(14)$ & $1.2158(16)$ \\
\hline $\mathrm{C}(1)-\mathrm{C}(2)$ & $1.5220(17)$ \\
\hline $\mathrm{C}(1)-\mathrm{C}(12)$ & $1.5313(17)$ \\
\hline $\mathrm{C}(1)-\mathrm{C}(20)$ & $1.5423(17)$ \\
\hline $\mathrm{C}(1)-\mathrm{H}(1)$ & 1.0000 \\
\hline$C(2)-C(3)$ & $1.3735(18)$ \\
\hline$C(2)-C(11)$ & $1.4342(17)$ \\
\hline$C(3)-C(4)$ & $1.4123(19)$ \\
\hline $\mathrm{C}(3)-\mathrm{H}(3)$ & 0.9500 \\
\hline$C(4)-C(5)$ & $1.3637(19)$ \\
\hline $\mathrm{C}(4)-\mathrm{H}(4)$ & 0.9500 \\
\hline$C(5)-C(6)$ & $1.4156(18)$ \\
\hline $\mathrm{C}(5)-\mathrm{H}(5)$ & 0.9500 \\
\hline$C(6)-C(7)$ & $1.4168(18)$ \\
\hline$C(6)-C(11)$ & $1.4273(17)$ \\
\hline$C(7)-C(8)$ & $1.366(2)$ \\
\hline $\mathrm{C}(7)-\mathrm{H}(7)$ & 0.9500 \\
\hline $\mathrm{C}(8)-\mathrm{C}(9)$ & 1.4091(19) \\
\hline $\mathrm{C}(8)-\mathrm{H}(8)$ & 0.9500 \\
\hline $\mathrm{C}(9)-\mathrm{C}(10)$ & $1.3702(18)$ \\
\hline $\mathrm{C}(9)-\mathrm{H}(9)$ & 0.9500 \\
\hline $\mathrm{C}(10)-\mathrm{C}(11)$ & $1.4230(18)$ \\
\hline $\mathrm{C}(10)-\mathrm{H}(10)$ & 0.9500 \\
\hline$C(12)-C(14)$ & $1.5189(18)$ \\
\hline $\mathrm{C}(12)-\mathrm{H}(12 \mathrm{~A})$ & 0.9900 \\
\hline $\mathrm{C}(12)-\mathrm{H}(12 \mathrm{~B})$ & 0.9900 \\
\hline $\mathrm{C}(13)-\mathrm{C}(19)$ & $1.3964(18)$ \\
\hline$C(13)-C(15)$ & $1.3973(18)$ \\
\hline $\mathrm{C}(13)-\mathrm{C}(14)$ & $1.4990(18)$ \\
\hline$C(15)-C(16)$ & $1.3886(19)$ \\
\hline $\mathrm{C}(15)-\mathrm{H}(15)$ & 0.9500 \\
\hline$C(16)-C(17)$ & $1.386(2)$ \\
\hline $\mathrm{C}(16)-\mathrm{H}(16)$ & 0.9500 \\
\hline $\mathrm{C}(17)-\mathrm{C}(18)$ & $1.389(2)$ \\
\hline $\mathrm{C}(17)-\mathrm{H}(17)$ & 0.9500 \\
\hline
\end{tabular}




\begin{tabular}{|c|c|}
\hline $\mathrm{C}(18)-\mathrm{C}(19)$ & $1.3849(19)$ \\
\hline C(18)-H(18) & 0.9500 \\
\hline $\mathrm{C}(19)-\mathrm{H}(19)$ & 0.9500 \\
\hline $\mathrm{C}(20)-\mathrm{C}(21)$ & $1.5142(17)$ \\
\hline $\mathrm{C}(20)-\mathrm{H}(20 \mathrm{~A})$ & 0.9900 \\
\hline $\mathrm{C}(20)-\mathrm{H}(20 \mathrm{~B})$ & 0.9900 \\
\hline $\mathrm{C}(21)-\mathrm{C}(22)$ & $1.4991(18)$ \\
\hline $\mathrm{C}(22)-\mathrm{C}(27)$ & $1.3951(18)$ \\
\hline $\mathrm{C}(22)-\mathrm{C}(23)$ & $1.3966(18)$ \\
\hline $\mathrm{C}(23)-\mathrm{C}(24)$ & $1.3889(19)$ \\
\hline $\mathrm{C}(23)-\mathrm{H}(23)$ & 0.9500 \\
\hline$C(24)-C(25)$ & $1.381(2)$ \\
\hline C(24)-H(24) & 0.9500 \\
\hline$C(25)-C(26)$ & $1.388(2)$ \\
\hline $\mathrm{C}(25)-\mathrm{H}(25)$ & 0.9500 \\
\hline $\mathrm{C}(26)-\mathrm{C}(27)$ & $1.3876(19)$ \\
\hline C(26)-H(26) & 0.9500 \\
\hline $\mathrm{C}(27)-\mathrm{H}(27)$ & 0.9500 \\
\hline $\mathrm{C}(2)-\mathrm{C}(1)-\mathrm{C}(12)$ & $113.58(10)$ \\
\hline $\mathrm{C}(2)-\mathrm{C}(1)-\mathrm{C}(20)$ & $108.89(10)$ \\
\hline$C(12)-C(1)-C(20)$ & $110.80(10)$ \\
\hline $\mathrm{C}(2)-\mathrm{C}(1)-\mathrm{H}(1)$ & 107.8 \\
\hline $\mathrm{C}(12)-\mathrm{C}(1)-\mathrm{H}(1)$ & 107.8 \\
\hline $\mathrm{C}(20)-\mathrm{C}(1)-\mathrm{H}(1)$ & 107.8 \\
\hline $\mathrm{C}(3)-\mathrm{C}(2)-\mathrm{C}(11)$ & $118.51(12)$ \\
\hline $\mathrm{C}(3)-\mathrm{C}(2)-\mathrm{C}(1)$ & $122.02(11)$ \\
\hline $\mathrm{C}(11)-\mathrm{C}(2)-\mathrm{C}(1)$ & $119.40(11)$ \\
\hline $\mathrm{C}(2)-\mathrm{C}(3)-\mathrm{C}(4)$ & $121.89(12)$ \\
\hline $\mathrm{C}(2)-\mathrm{C}(3)-\mathrm{H}(3)$ & 119.1 \\
\hline $\mathrm{C}(4)-\mathrm{C}(3)-\mathrm{H}(3)$ & 119.1 \\
\hline $\mathrm{C}(5)-\mathrm{C}(4)-\mathrm{C}(3)$ & $120.44(12)$ \\
\hline $\mathrm{C}(5)-\mathrm{C}(4)-\mathrm{H}(4)$ & 119.8 \\
\hline $\mathrm{C}(3)-\mathrm{C}(4)-\mathrm{H}(4)$ & 119.8 \\
\hline$C(4)-C(5)-C(6)$ & $120.22(12)$ \\
\hline $\mathrm{C}(4)-\mathrm{C}(5)-\mathrm{H}(5)$ & 119.9 \\
\hline $\mathrm{C}(6)-\mathrm{C}(5)-\mathrm{H}(5)$ & 119.9 \\
\hline$C(5)-C(6)-C(7)$ & $120.84(12)$ \\
\hline$C(5)-C(6)-C(11)$ & $119.45(12)$ \\
\hline
\end{tabular}




\begin{tabular}{|c|c|}
\hline$C(7)-C(6)-C(11)$ & $119.70(12)$ \\
\hline$C(8)-C(7)-C(6)$ & $120.90(13)$ \\
\hline $\mathrm{C}(8)-\mathrm{C}(7)-\mathrm{H}(7)$ & 119.6 \\
\hline $\mathrm{C}(6)-\mathrm{C}(7)-\mathrm{H}(7)$ & 119.6 \\
\hline $\mathrm{C}(7)-\mathrm{C}(8)-\mathrm{C}(9)$ & $119.99(13)$ \\
\hline $\mathrm{C}(7)-\mathrm{C}(8)-\mathrm{H}(8)$ & 120.0 \\
\hline $\mathrm{C}(9)-\mathrm{C}(8)-\mathrm{H}(8)$ & 120.0 \\
\hline $\mathrm{C}(10)-\mathrm{C}(9)-\mathrm{C}(8)$ & $120.46(12)$ \\
\hline $\mathrm{C}(10)-\mathrm{C}(9)-\mathrm{H}(9)$ & 119.8 \\
\hline $\mathrm{C}(8)-\mathrm{C}(9)-\mathrm{H}(9)$ & 119.8 \\
\hline $\mathrm{C}(9)-\mathrm{C}(10)-\mathrm{C}(11)$ & $121.43(12)$ \\
\hline $\mathrm{C}(9)-\mathrm{C}(10)-\mathrm{H}(10)$ & 119.3 \\
\hline $\mathrm{C}(11)-\mathrm{C}(10)-\mathrm{H}(10)$ & 119.3 \\
\hline $\mathrm{C}(10)-\mathrm{C}(11)-\mathrm{C}(6)$ & $117.51(11)$ \\
\hline $\mathrm{C}(10)-\mathrm{C}(11)-\mathrm{C}(2)$ & $123.02(11)$ \\
\hline $\mathrm{C}(6)-\mathrm{C}(11)-\mathrm{C}(2)$ & $119.45(11)$ \\
\hline $\mathrm{C}(14)-\mathrm{C}(12)-\mathrm{C}(1)$ & $112.63(10)$ \\
\hline $\mathrm{C}(14)-\mathrm{C}(12)-\mathrm{H}(12 \mathrm{~A})$ & 109.1 \\
\hline $\mathrm{C}(1)-\mathrm{C}(12)-\mathrm{H}(12 \mathrm{~A})$ & 109.1 \\
\hline $\mathrm{C}(14)-\mathrm{C}(12)-\mathrm{H}(12 \mathrm{~B})$ & 109.1 \\
\hline $\mathrm{C}(1)-\mathrm{C}(12)-\mathrm{H}(12 \mathrm{~B})$ & 109.1 \\
\hline $\mathrm{H}(12 \mathrm{~A})-\mathrm{C}(12)-\mathrm{H}(12 \mathrm{~B})$ & 107.8 \\
\hline $\mathrm{C}(19)-\mathrm{C}(13)-\mathrm{C}(15)$ & $119.15(12)$ \\
\hline$C(19)-C(13)-C(14)$ & $118.41(11)$ \\
\hline $\mathrm{C}(15)-\mathrm{C}(13)-\mathrm{C}(14)$ & $122.44(12)$ \\
\hline $\mathrm{O}(2)-\mathrm{C}(14)-\mathrm{C}(13)$ & $120.14(12)$ \\
\hline $\mathrm{O}(2)-\mathrm{C}(14)-\mathrm{C}(12)$ & $120.95(12)$ \\
\hline$C(13)-C(14)-C(12)$ & $118.91(11)$ \\
\hline $\mathrm{C}(16)-\mathrm{C}(15)-\mathrm{C}(13)$ & $120.10(12)$ \\
\hline $\mathrm{C}(16)-\mathrm{C}(15)-\mathrm{H}(15)$ & 119.9 \\
\hline $\mathrm{C}(13)-\mathrm{C}(15)-\mathrm{H}(15)$ & 119.9 \\
\hline$C(17)-C(16)-C(15)$ & $120.18(13)$ \\
\hline $\mathrm{C}(17)-\mathrm{C}(16)-\mathrm{H}(16)$ & 119.9 \\
\hline $\mathrm{C}(15)-\mathrm{C}(16)-\mathrm{H}(16)$ & 119.9 \\
\hline$C(16)-C(17)-C(18)$ & $120.15(13)$ \\
\hline $\mathrm{C}(16)-\mathrm{C}(17)-\mathrm{H}(17)$ & 119.9 \\
\hline $\mathrm{C}(18)-\mathrm{C}(17)-\mathrm{H}(17)$ & 119.9 \\
\hline$C(19)-C(18)-C(17)$ & $119.81(13)$ \\
\hline
\end{tabular}




$\begin{array}{ll}\mathrm{C}(19)-\mathrm{C}(18)-\mathrm{H}(18) & 120.1 \\ \mathrm{C}(17)-\mathrm{C}(18)-\mathrm{H}(18) & 120.1 \\ \mathrm{C}(18)-\mathrm{C}(19)-\mathrm{C}(13) & 120.60(12) \\ \mathrm{C}(18)-\mathrm{C}(19)-\mathrm{H}(19) & 119.7 \\ \mathrm{C}(13)-\mathrm{C}(19)-\mathrm{H}(19) & 119.7 \\ \mathrm{C}(21)-\mathrm{C}(20)-\mathrm{C}(1) & 114.97(10) \\ \mathrm{C}(21)-\mathrm{C}(20)-\mathrm{H}(20 A) & 108.5 \\ \mathrm{C}(1)-\mathrm{C}(20)-\mathrm{H}(20 A) & 108.5 \\ \mathrm{C}(21)-\mathrm{C}(20)-\mathrm{H}(20 \mathrm{~B}) & 108.5 \\ \mathrm{C}(1)-\mathrm{C}(20)-\mathrm{H}(20 \mathrm{~B}) & 108.5 \\ \mathrm{H}(20 \mathrm{~A})-\mathrm{C}(20)-\mathrm{H}(20 \mathrm{~B}) & 107.5 \\ \mathrm{O}(1)-\mathrm{C}(21)-\mathrm{C}(22) & 120.69(11) \\ \mathrm{O}(1)-\mathrm{C}(21)-\mathrm{C}(20) & 121.69(11) \\ \mathrm{C}(22)-\mathrm{C}(21)-\mathrm{C}(20) & 117.60(11) \\ \mathrm{C}(27)-\mathrm{C}(22)-\mathrm{C}(23) & 119.21(12) \\ \mathrm{C}(27)-\mathrm{C}(22)-\mathrm{C}(21) & 118.74(11) \\ \mathrm{C}(23)-\mathrm{C}(22)-\mathrm{C}(21) & 122.01(11) \\ \mathrm{C}(24)-\mathrm{C}(23)-\mathrm{C}(22) & 120.27(12) \\ \mathrm{C}(24)-\mathrm{C}(23)-\mathrm{H}(23) & 119.9 \\ \mathrm{C}(22)-\mathrm{C}(23)-\mathrm{H}(23) & 119.9 \\ \mathrm{C}(25)-\mathrm{C}(24)-\mathrm{C}(23) & 119.94(13) \\ \mathrm{C}(25)-\mathrm{C}(24)-\mathrm{H}(24) & 120.0 \\ \mathrm{C}(23)-\mathrm{C}(24)-\mathrm{H}(24) & 120.0 \\ \mathrm{C}(24)-\mathrm{C}(25)-\mathrm{C}(26) & 120.40(13) \\ \mathrm{C}(24)-\mathrm{C}(25)-\mathrm{H}(25) & 119.8 \\ \mathrm{C}(26)-\mathrm{C}(25)-\mathrm{H}(25) & 119.8 \\ \mathrm{C}(27)-\mathrm{C}(26)-\mathrm{C}(25) & 119.87(13) \\ \mathrm{C}(27)-\mathrm{C}(26)-\mathrm{H}(26) & 120.1 \\ \mathrm{C}(25)-\mathrm{C}(26)-\mathrm{H}(26) & 120.1 \\ \mathrm{C}(26)-\mathrm{C}(27)-\mathrm{C}(22) & 120.26(13) \\ \mathrm{C}(26)-\mathrm{C}(27)-\mathrm{H}(27) & 119.9 \\ \mathrm{C}(22)-\mathrm{C}(27)-\mathrm{H}(27) & 119.9 \\ & \end{array}$

Symmetry transformations used to generate equivalent atoms: 
Table S12. Anisotropic displacement parameters $\left(\AA^{2} \times 10^{3}\right)$ for i18009. The anisotropic displacement factor exponent takes the form: $-2 \pi^{2}\left[h^{2} a^{* 2} U^{11}+\ldots+2 h k a^{*} b^{*} U^{12}\right]$

\begin{tabular}{|c|c|c|c|c|c|c|}
\hline & $\mathrm{U}^{11}$ & $\mathrm{U}^{22}$ & $\mathrm{U}^{33}$ & $\mathrm{U}^{23}$ & $\mathrm{U}^{13}$ & $\mathrm{U}^{12}$ \\
\hline $\mathrm{O}(1)$ & $20(1)$ & $22(1)$ & $27(1)$ & $2(1)$ & $0(1)$ & 2(1) \\
\hline $\mathrm{O}(2)$ & $55(1)$ & $22(1)$ & $20(1)$ & $-2(1)$ & $4(1)$ & $9(1)$ \\
\hline$C(1)$ & $20(1)$ & $17(1)$ & $17(1)$ & $1(1)$ & $2(1)$ & $0(1)$ \\
\hline $\mathrm{C}(2)$ & 21(1) & $13(1)$ & $17(1)$ & $3(1)$ & $1(1)$ & 1(1) \\
\hline$C(3)$ & $24(1)$ & $21(1)$ & $16(1)$ & $3(1)$ & $3(1)$ & 1(1) \\
\hline $\mathrm{C}(4)$ & $19(1)$ & $23(1)$ & $24(1)$ & $7(1)$ & $7(1)$ & 1(1) \\
\hline$C(5)$ & $18(1)$ & $18(1)$ & $25(1)$ & $5(1)$ & $0(1)$ & $-2(1)$ \\
\hline$C(6)$ & $20(1)$ & $14(1)$ & $20(1)$ & $3(1)$ & $0(1)$ & 1(1) \\
\hline$C(7)$ & $23(1)$ & $21(1)$ & $22(1)$ & $-1(1)$ & $-2(1)$ & $-1(1)$ \\
\hline$C(8)$ & $30(1)$ & $28(1)$ & $18(1)$ & $-4(1)$ & $1(1)$ & 1(1) \\
\hline $\mathrm{C}(9)$ & $23(1)$ & $26(1)$ & $21(1)$ & $-1(1)$ & $6(1)$ & 1(1) \\
\hline$C(10)$ & $19(1)$ & $18(1)$ & $20(1)$ & $0(1)$ & $2(1)$ & $0(1)$ \\
\hline$C(11)$ & $18(1)$ & $13(1)$ & $18(1)$ & $2(1)$ & $1(1)$ & 1(1) \\
\hline$C(12)$ & $22(1)$ & $18(1)$ & $18(1)$ & $-1(1)$ & $0(1)$ & $0(1)$ \\
\hline$C(13)$ & $14(1)$ & $21(1)$ & $18(1)$ & $0(1)$ & $-2(1)$ & 1(1) \\
\hline$C(14)$ & $21(1)$ & $20(1)$ & $19(1)$ & $-1(1)$ & $-2(1)$ & 1(1) \\
\hline$C(15)$ & $19(1)$ & $20(1)$ & $21(1)$ & $0(1)$ & $0(1)$ & $0(1)$ \\
\hline$C(16)$ & $25(1)$ & $25(1)$ & $21(1)$ & $-3(1)$ & $4(1)$ & $2(1)$ \\
\hline$C(17)$ & $24(1)$ & $31(1)$ & $20(1)$ & $4(1)$ & $5(1)$ & $2(1)$ \\
\hline$C(18)$ & $21(1)$ & $23(1)$ & $26(1)$ & $6(1)$ & 1(1) & 1(1) \\
\hline$C(19)$ & $18(1)$ & $20(1)$ & $22(1)$ & $0(1)$ & $-1(1)$ & 1(1) \\
\hline$C(20)$ & $19(1)$ & $18(1)$ & $18(1)$ & $1(1)$ & $0(1)$ & $0(1)$ \\
\hline$C(21)$ & 19(1) & $20(1)$ & $15(1)$ & $-2(1)$ & $3(1)$ & $1(1)$ \\
\hline$C(22)$ & $20(1)$ & $19(1)$ & $15(1)$ & $-1(1)$ & $5(1)$ & $0(1)$ \\
\hline$C(23)$ & 19(1) & $22(1)$ & $20(1)$ & $1(1)$ & $3(1)$ & $-1(1)$ \\
\hline$C(24)$ & $24(1)$ & $24(1)$ & $25(1)$ & $5(1)$ & $4(1)$ & $2(1)$ \\
\hline$C(25)$ & $31(1)$ & $19(1)$ & $27(1)$ & $3(1)$ & $5(1)$ & $-2(1)$ \\
\hline$C(26)$ & $27(1)$ & $26(1)$ & $31(1)$ & 1(1) & $-2(1)$ & $-8(1)$ \\
\hline$C(27)$ & 21(1) & $24(1)$ & $25(1)$ & $2(1)$ & $-1(1)$ & $-2(1)$ \\
\hline
\end{tabular}




\section{Crystal data and structure refinement for compound $\mathbf{4 e}$}

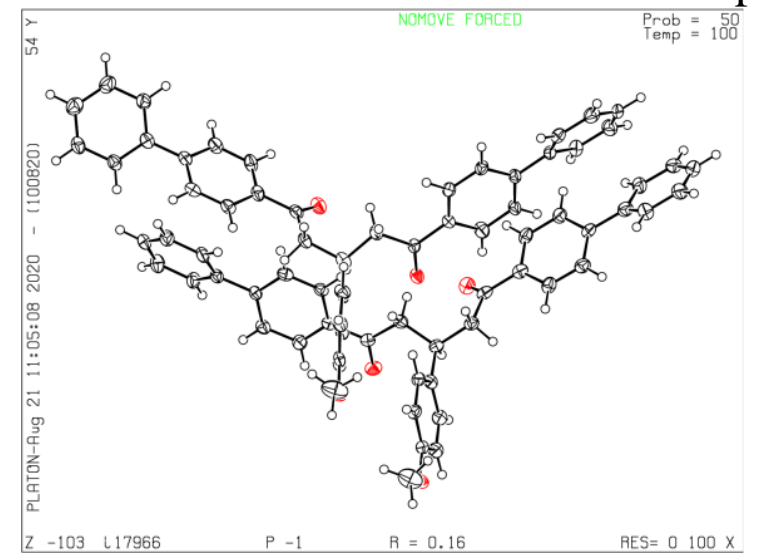

Figure S4. Single X-ray crystal structure of 1, 5-dicarbonyls 4e (the thermal ellipsoid was drawn at the 50\% probability level).

Table S13. Crystal data and structure refinement for i17966.

CCDC

Identification code

Empirical formula

Formula weight

Temperature

Wavelength

Crystal system

Space group

Unit cell dimensions

Z, Volume

Density (calculated)

Absorption coefficient

$\mathrm{F}(000)$

Crystal size

Theta range for data collection

Index ranges

Reflections collected

Independent reflections

Completeness to theta $=25.242^{\circ}$

Absorption correction

Max. and min. transmission

Refinement method

Data / restraints / parameters

Goodness-of-fit on $\mathrm{F}^{2}$
2031242

i17966

C36 H30 O3

510.60

100.0(2) K

$0.71073 \AA$

Triclinic

P -1

$a=10.2451(10) \AA \quad \alpha=89.086(4)^{\circ}$.

$\mathrm{b}=10.3941(9) \AA \quad \beta=89.020(4)^{\circ}$.

$\mathrm{c}=26.429(2) \AA$ $\gamma=77.645(4)^{\circ}$.

4, 2748.6(4) $\AA^{3}$

$1.234 \mathrm{Mg} / \mathrm{m}^{3}$

$0.077 \mathrm{~mm}^{-1}$

1080

$0.167 \times 0.110 \times 0.063 \mathrm{~mm}^{3}$

2.035 to $27.193^{\circ}$.

$-13<=\mathrm{h}<=13,-13<=\mathrm{k}<=13,-33<=1<=33$

132589

$12176[\mathrm{R}$ (int) $=0.1739]$

$99.9 \%$

Numerical

0.9787 and 0.8859

Full-matrix least-squares on $\mathrm{F}^{2}$

12176 / 0 / 706

1.091 
Final $\mathrm{R}$ indices [I $>2 \operatorname{sigma}(\mathrm{I})]$

$\mathrm{R}$ indices (all data)

Extinction coefficient

Largest diff. peak and hole
$\mathrm{R} 1=0.1599, \mathrm{wR} 2=0.4169$

$\mathrm{R} 1=0.2009, \mathrm{wR} 2=0.4372$

$0.0104(15)$

0.739 and -0.558 e. $\AA^{-3}$

Table S14. Atomic coordinates ( $\left.x \mathbf{1 0}^{4}\right)$ and equivalent isotropic displacement parameters $\left(\AA^{2} \times 1^{3}\right)$ for i17966. $U(\mathrm{eq})$ is defined as one third of the trace of the orthogonalized $U$ ij tensor.

\begin{tabular}{|c|c|c|c|c|}
\hline & $\mathrm{X}$ & $\mathrm{y}$ & $\mathrm{z}$ & $\mathrm{U}(\mathrm{eq})$ \\
\hline $\mathrm{O}(1)$ & $7207(6)$ & $-901(6)$ & $8007(2)$ & $38(2)$ \\
\hline $\mathrm{O}(2)$ & $4654(5)$ & $4821(6)$ & $7034(2)$ & $27(1)$ \\
\hline $\mathrm{O}(3)$ & $5075(6)$ & $7312(6)$ & $8306(2)$ & $32(1)$ \\
\hline $\mathrm{O}(4)$ & $2064(6)$ & $-1085(6)$ & $7186(2)$ & $36(1)$ \\
\hline $\mathrm{O}(5)$ & $319(6)$ & $7064(6)$ & $6559(2)$ & $32(1)$ \\
\hline $\mathrm{O}(6)$ & $-420(5)$ & $4682(6)$ & $7870(2)$ & $30(1)$ \\
\hline $\mathrm{C}(1)$ & $5943(7)$ & $4716(8)$ & $7953(3)$ & $27(2)$ \\
\hline $\mathrm{C}(2)$ & $6296(7)$ & $3211(8)$ & $7950(3)$ & $21(2)$ \\
\hline $\mathrm{C}(3)$ & $7632(8)$ & $2530(8)$ & $7902(3)$ & $29(2)$ \\
\hline $\mathrm{C}(4)$ & $7974(8)$ & $1159(8)$ & $7932(3)$ & $29(2)$ \\
\hline $\mathrm{C}(5)$ & $6990(8)$ & 461(8) & 7993(3) & $26(2)$ \\
\hline$C(6)$ & $5652(8)$ & $1112(8)$ & $8054(3)$ & $28(2)$ \\
\hline$C(7)$ & $5345(7)$ & $2466(8)$ & $8023(3)$ & $23(2)$ \\
\hline $\mathrm{C}(8)$ & $8545(11)$ & $-1610(10)$ & $7999(5)$ & $58(3)$ \\
\hline $\mathrm{C}(9)$ & $6453(8)$ & $5361(8)$ & $7482(3)$ & $26(2)$ \\
\hline$C(10)$ & $5630(7)$ & $5320(7)$ & $7018(3)$ & $20(1)$ \\
\hline $\mathrm{C}(11)$ & $6023(7)$ & $5904(7)$ & $6531(3)$ & $21(2)$ \\
\hline$C(12)$ & $7214(8)$ & $6330(8)$ & $6465(3)$ & $26(2)$ \\
\hline$C(13)$ & $7528(8)$ & $6869(8)$ & $6007(3)$ & $25(2)$ \\
\hline$C(14)$ & $6634(8)$ & $7059(7)$ & $5606(3)$ & $23(2)$ \\
\hline$C(15)$ & $5438(8)$ & $6655(8)$ & $5674(3)$ & $28(2)$ \\
\hline$C(16)$ & $5136(8)$ & $6078(9)$ & $6132(3)$ & $31(2)$ \\
\hline$C(17)$ & $6979(8)$ & $7638(7)$ & $5117(3)$ & $24(2)$ \\
\hline$C(18)$ & $8312(8)$ & $7418(8)$ & $4940(3)$ & $27(2)$ \\
\hline C(19) & $8616(9)$ & $7916(9)$ & $4474(3)$ & $32(2)$ \\
\hline$C(20)$ & 7607(9) & $8639(9)$ & $4169(3)$ & $34(2)$ \\
\hline$C(21)$ & $6294(9)$ & $8870(9)$ & $4341(3)$ & $34(2)$ \\
\hline $\mathrm{C}(22)$ & $5965(8)$ & $8383(8)$ & $4812(3)$ & $29(2)$ \\
\hline $\mathrm{C}(23)$ & $6474(8)$ & $5196(8)$ & $8437(3)$ & $25(2)$ \\
\hline
\end{tabular}




\begin{tabular}{|c|c|c|c|c|}
\hline$C(24)$ & $5892(8)$ & $6621(8)$ & $8568(3)$ & $26(2)$ \\
\hline$C(25)$ & 6301(8) & $7119(8)$ & $9057(3)$ & $27(2)$ \\
\hline$C(26)$ & $5975(9)$ & $8439(9)$ & 9154(3) & $32(2)$ \\
\hline $\mathrm{C}(27)$ & $6326(8)$ & $8930(8)$ & $9604(3)$ & $29(2)$ \\
\hline$C(28)$ & $7017(8)$ & $8082(8)$ & 9982(3) & $24(2)$ \\
\hline $\mathrm{C}(29)$ & $7318(8)$ & 6734(8) & $9879(3)$ & $27(2)$ \\
\hline $\mathrm{C}(30)$ & $6985(8)$ & $6257(8)$ & $9426(3)$ & $26(2)$ \\
\hline $\mathrm{C}(31)$ & $7396(8)$ & $8574(8)$ & $10468(3)$ & $26(2)$ \\
\hline $\mathrm{C}(32)$ & $7863(10)$ & $9725(8)$ & 10481(3) & $34(2)$ \\
\hline $\mathrm{C}(33)$ & $8249(10)$ & 10171(9) & 10941(4) & $39(2)$ \\
\hline$C(34)$ & $8210(10)$ & $9460(9)$ & $11388(4)$ & $38(2)$ \\
\hline$C(35)$ & 7779(9) & $8289(9)$ & 11373(3) & $32(2)$ \\
\hline$C(36)$ & $7376(8)$ & $7842(8)$ & 10911(3) & $28(2)$ \\
\hline $\mathrm{C}(37)$ & $995(7)$ & $4493(7)$ & $6960(3)$ & $23(2)$ \\
\hline $\mathrm{C}(38)$ & $1317(7)$ & 2997(8) & 7033(3) & $22(2)$ \\
\hline$C(39)$ & $2599(7)$ & 2314(7) & 7139(3) & $23(2)$ \\
\hline $\mathrm{C}(40)$ & 2898(8) & $942(8)$ & $7188(3)$ & $24(2)$ \\
\hline $\mathrm{C}(41)$ & 1907(8) & $244(7)$ & 7141(3) & $23(2)$ \\
\hline $\mathrm{C}(42)$ & $612(8)$ & $916(8)$ & 7021(3) & $28(2)$ \\
\hline$C(43)$ & $328(8)$ & $2283(8)$ & 6971(3) & $27(2)$ \\
\hline $\mathrm{C}(44)$ & $3405(11)$ & $-1812(10)$ & $7248(5)$ & $53(3)$ \\
\hline $\mathrm{C}(45)$ & $1604(8)$ & $4877(7)$ & $6456(3)$ & $25(2)$ \\
\hline $\mathrm{C}(46)$ & $1089(8)$ & 6293(8) & $6293(3)$ & $26(2)$ \\
\hline $\mathrm{C}(47)$ & $1535(8)$ & $6742(7)$ & $5792(3)$ & $25(2)$ \\
\hline $\mathrm{C}(48)$ & 1981(8) & $5897(8)$ & 5394(3) & $29(2)$ \\
\hline $\mathrm{C}(49)$ & $2279(8)$ & 6361(8) & 4926(3) & $29(2)$ \\
\hline $\mathrm{C}(50)$ & 2193(8) & 7694(8) & 4834(3) & $26(2)$ \\
\hline $\mathrm{C}(51)$ & 1778(9) & $8556(8)$ & $5235(3)$ & $29(2)$ \\
\hline$C(52)$ & $1447(8)$ & $8081(8)$ & $5708(3)$ & $28(2)$ \\
\hline$C(53)$ & 2497(8) & $8216(8)$ & $4327(3)$ & $30(2)$ \\
\hline$C(54)$ & $3462(9)$ & 7497(9) & 4011(3) & $32(2)$ \\
\hline$C(55)$ & $3750(10)$ & 7991(10) & $3538(3)$ & $40(2)$ \\
\hline$C(56)$ & $3090(10)$ & $9209(10)$ & $3383(3)$ & $39(2)$ \\
\hline $\mathrm{C}(57)$ & $2110(10)$ & 9962(9) & $3684(3)$ & $37(2)$ \\
\hline $\mathrm{C}(58)$ & 1831(9) & $9475(8)$ & $4160(3)$ & $32(2)$ \\
\hline $\mathrm{C}(59)$ & $1431(8)$ & $5186(8)$ & $7409(3)$ & $26(2)$ \\
\hline $\mathrm{C}(60)$ & $614(7)$ & $5082(7)$ & 7889(3) & $23(2)$ \\
\hline $\mathrm{C}(61)$ & $1077(7)$ & $5463(7)$ & $8381(3)$ & $23(2)$ \\
\hline
\end{tabular}




\begin{tabular}{lrlll}
$\mathrm{C}(62)$ & $2173(8)$ & $6039(7)$ & $8426(3)$ & $23(2)$ \\
$\mathrm{C}(63)$ & $2514(8)$ & $6462(8)$ & $8885(3)$ & $27(2)$ \\
$\mathrm{C}(64)$ & $1761(7)$ & $6299(7)$ & $9321(3)$ & $21(2)$ \\
$\mathrm{C}(65)$ & $667(7)$ & $5722(8)$ & $9281(3)$ & $25(2)$ \\
$\mathrm{C}(66)$ & $321(8)$ & $5305(8)$ & $8812(3)$ & $27(2)$ \\
$\mathrm{C}(67)$ & $2105(7)$ & $6776(8)$ & $9818(3)$ & $24(2)$ \\
$\mathrm{C}(68)$ & $3446(8)$ & $6743(8)$ & $9940(3)$ & $28(2)$ \\
$\mathrm{C}(69)$ & $3751(9)$ & $7232(9)$ & $10397(3)$ & $31(2)$ \\
$\mathrm{C}(70)$ & $2761(9)$ & $7755(8)$ & $10745(3)$ & $31(2)$ \\
$\mathrm{C}(71)$ & $1425(9)$ & $7768(9)$ & $10634(3)$ & $35(2)$ \\
$\mathrm{C}(72)$ & $1116(8)$ & $7279(8)$ & $10175(3)$ & $28(2)$ \\
\hline
\end{tabular}


Table S15. Bond lengths $[\AA ̊ \AA]$ and angles $\left[{ }^{\circ}\right]$ for i17966.

\begin{tabular}{|c|c|}
\hline $\mathrm{O}(1)-\mathrm{C}(5)$ & $1.385(10)$ \\
\hline $\mathrm{O}(1)-\mathrm{C}(8)$ & $1.410(12)$ \\
\hline $\mathrm{O}(2)-\mathrm{C}(10)$ & $1.221(9)$ \\
\hline $\mathrm{O}(3)-\mathrm{C}(24)$ & $1.201(10)$ \\
\hline $\mathrm{O}(4)-\mathrm{C}(41)$ & $1.359(9)$ \\
\hline $\mathrm{O}(4)-\mathrm{C}(44)$ & $1.430(11)$ \\
\hline $\mathrm{O}(5)-\mathrm{C}(46)$ & $1.220(10)$ \\
\hline $\mathrm{O}(6)-\mathrm{C}(60)$ & $1.220(9)$ \\
\hline $\mathrm{C}(1)-\mathrm{C}(2)$ & $1.528(11)$ \\
\hline C(1)-C(23) & $1.530(11)$ \\
\hline $\mathrm{C}(1)-\mathrm{C}(9)$ & $1.540(10)$ \\
\hline $\mathrm{C}(1)-\mathrm{H}(1)$ & 1.0000 \\
\hline$C(2)-C(7)$ & $1.378(10)$ \\
\hline$C(2)-C(3)$ & $1.405(10)$ \\
\hline$C(3)-C(4)$ & $1.394(12)$ \\
\hline $\mathrm{C}(3)-\mathrm{H}(3)$ & 0.9500 \\
\hline $\mathrm{C}(4)-\mathrm{C}(5)$ & $1.370(11)$ \\
\hline $\mathrm{C}(4)-\mathrm{H}(4)$ & 0.9500 \\
\hline $\mathrm{C}(5)-\mathrm{C}(6)$ & $1.401(11)$ \\
\hline$C(6)-C(7)$ & $1.376(11)$ \\
\hline $\mathrm{C}(6)-\mathrm{H}(6)$ & 0.9500 \\
\hline $\mathrm{C}(7)-\mathrm{H}(7)$ & 0.9500 \\
\hline $\mathrm{C}(8)-\mathrm{H}(8 \mathrm{~A})$ & 0.9800 \\
\hline $\mathrm{C}(8)-\mathrm{H}(8 \mathrm{~B})$ & 0.9800 \\
\hline $\mathrm{C}(8)-\mathrm{H}(8 \mathrm{C})$ & 0.9800 \\
\hline$C(9)-C(10)$ & $1.506(10)$ \\
\hline $\mathrm{C}(9)-\mathrm{H}(9 \mathrm{~A})$ & 0.9900 \\
\hline $\mathrm{C}(9)-\mathrm{H}(9 \mathrm{AB})$ & 0.9900 \\
\hline$C(10)-C(11)$ & $1.501(10)$ \\
\hline$C(11)-C(16)$ & $1.387(11)$ \\
\hline $\mathrm{C}(11)-\mathrm{C}(12)$ & $1.392(10)$ \\
\hline$C(12)-C(13)$ & $1.385(11)$ \\
\hline $\mathrm{C}(12)-\mathrm{H}(12)$ & 0.9500 \\
\hline$C(13)-C(14)$ & $1.398(10)$ \\
\hline $\mathrm{C}(13)-\mathrm{H}(13)$ & 0.9500 \\
\hline$C(14)-C(15)$ & $1.385(11)$ \\
\hline
\end{tabular}




\begin{tabular}{|c|c|}
\hline C(14)-C(17) & $1.487(10)$ \\
\hline$C(15)-C(16)$ & $1.403(11)$ \\
\hline $\mathrm{C}(15)-\mathrm{H}(15)$ & 0.9500 \\
\hline $\mathrm{C}(16)-\mathrm{H}(16)$ & 0.9500 \\
\hline $\mathrm{C}(17)-\mathrm{C}(18)$ & $1.408(11)$ \\
\hline $\mathrm{C}(17)-\mathrm{C}(22)$ & $1.411(11)$ \\
\hline $\mathrm{C}(18)-\mathrm{C}(19)$ & $1.384(12)$ \\
\hline $\mathrm{C}(18)-\mathrm{H}(18)$ & 0.9500 \\
\hline $\mathrm{C}(19)-\mathrm{C}(20)$ & $1.400(13)$ \\
\hline C(19)-H(19) & 0.9500 \\
\hline $\mathrm{C}(20)-\mathrm{C}(21)$ & $1.385(13)$ \\
\hline $\mathrm{C}(20)-\mathrm{H}(20)$ & 0.9500 \\
\hline $\mathrm{C}(21)-\mathrm{C}(22)$ & $1.400(12)$ \\
\hline $\mathrm{C}(21)-\mathrm{H}(21)$ & 0.9500 \\
\hline $\mathrm{C}(22)-\mathrm{H}(22)$ & 0.9500 \\
\hline $\mathrm{C}(23)-\mathrm{C}(24)$ & $1.517(11)$ \\
\hline $\mathrm{C}(23)-\mathrm{H}(23 \mathrm{~A})$ & 0.9900 \\
\hline $\mathrm{C}(23)-\mathrm{H}(23 \mathrm{~B})$ & 0.9900 \\
\hline $\mathrm{C}(24)-\mathrm{C}(25)$ & $1.499(11)$ \\
\hline $\mathrm{C}(25)-\mathrm{C}(26)$ & $1.368(12)$ \\
\hline $\mathrm{C}(25)-\mathrm{C}(30)$ & $1.404(11)$ \\
\hline $\mathrm{C}(26)-\mathrm{C}(27)$ & $1.385(12)$ \\
\hline $\mathrm{C}(26)-\mathrm{H}(26)$ & 0.9500 \\
\hline $\mathrm{C}(27)-\mathrm{C}(28)$ & $1.417(11)$ \\
\hline $\mathrm{C}(27)-\mathrm{H}(27)$ & 0.9500 \\
\hline $\mathrm{C}(28)-\mathrm{C}(29)$ & $1.400(11)$ \\
\hline $\mathrm{C}(28)-\mathrm{C}(31)$ & $1.478(11)$ \\
\hline $\mathrm{C}(29)-\mathrm{C}(30)$ & $1.378(11)$ \\
\hline $\mathrm{C}(29)-\mathrm{H}(29)$ & 0.9500 \\
\hline $\mathrm{C}(30)-\mathrm{H}(30)$ & 0.9500 \\
\hline $\mathrm{C}(31)-\mathrm{C}(32)$ & $1.381(12)$ \\
\hline $\mathrm{C}(31)-\mathrm{C}(36)$ & $1.387(11)$ \\
\hline $\mathrm{C}(32)-\mathrm{C}(33)$ & $1.400(12)$ \\
\hline $\mathrm{C}(32)-\mathrm{H}(32)$ & 0.9500 \\
\hline $\mathrm{C}(33)-\mathrm{C}(34)$ & $1.388(13)$ \\
\hline $\mathrm{C}(33)-\mathrm{H}(33)$ & 0.9500 \\
\hline $\mathrm{C}(34)-\mathrm{C}(35)$ & $1.382(13)$ \\
\hline $\mathrm{C}(34)-\mathrm{H}(34)$ & 0.9500 \\
\hline
\end{tabular}




\begin{tabular}{|c|c|}
\hline$C(35)-C(36)$ & $1.413(12)$ \\
\hline C(35)-H(35) & 0.9500 \\
\hline $\mathrm{C}(36)-\mathrm{H}(36)$ & 0.9500 \\
\hline C(37)-C(59) & $1.519(11)$ \\
\hline $\mathrm{C}(37)-\mathrm{C}(38)$ & $1.528(11)$ \\
\hline C(37)-C(45) & $1.545(11)$ \\
\hline C(37)-H(37) & 1.0000 \\
\hline C(38)-C(39) & $1.384(10)$ \\
\hline C(38)-C(43) & $1.391(10)$ \\
\hline C(39)-C(40) & $1.397(11)$ \\
\hline C(39)-H(39) & 0.9500 \\
\hline $\mathrm{C}(40)-\mathrm{C}(41)$ & $1.377(10)$ \\
\hline $\mathrm{C}(40)-\mathrm{H}(40)$ & 0.9500 \\
\hline $\mathrm{C}(41)-\mathrm{C}(42)$ & $1.400(11)$ \\
\hline C(42)-C(43) & $1.393(12)$ \\
\hline $\mathrm{C}(42)-\mathrm{H}(42)$ & 0.9500 \\
\hline C(43)-H(43) & 0.9500 \\
\hline $\mathrm{C}(44)-\mathrm{H}(44 \mathrm{~A})$ & 0.9800 \\
\hline C(44)-H(44B) & 0.9800 \\
\hline $\mathrm{C}(44)-\mathrm{H}(44 \mathrm{C})$ & 0.9800 \\
\hline $\mathrm{C}(45)-\mathrm{C}(46)$ & $1.512(11)$ \\
\hline $\mathrm{C}(45)-\mathrm{H}(45 \mathrm{~A})$ & 0.9900 \\
\hline $\mathrm{C}(45)-\mathrm{H}(45 \mathrm{~B})$ & 0.9900 \\
\hline C(46)-C(47) & $1.494(11)$ \\
\hline C(47)-C(48) & $1.387(11)$ \\
\hline $\mathrm{C}(47)-\mathrm{C}(52)$ & $1.389(11)$ \\
\hline $\mathrm{C}(48)-\mathrm{C}(49)$ & $1.375(12)$ \\
\hline C(48)-H(48) & 0.9500 \\
\hline C(49)-C(50) & $1.387(11)$ \\
\hline C(49)-H(49) & 0.9500 \\
\hline $\mathrm{C}(50)-\mathrm{C}(51)$ & $1.400(11)$ \\
\hline $\mathrm{C}(50)-\mathrm{C}(53)$ & $1.489(11)$ \\
\hline $\mathrm{C}(51)-\mathrm{C}(52)$ & $1.400(11)$ \\
\hline $\mathrm{C}(51)-\mathrm{H}(51)$ & 0.9500 \\
\hline $\mathrm{C}(52)-\mathrm{H}(52)$ & 0.9500 \\
\hline $\mathrm{C}(53)-\mathrm{C}(54)$ & $1.382(12)$ \\
\hline C(53)-C(58) & $1.408(11)$ \\
\hline$C(54)-C(55)$ & $1.396(12)$ \\
\hline
\end{tabular}




\begin{tabular}{|c|c|}
\hline $\mathrm{C}(54)-\mathrm{H}(54)$ & 0.9500 \\
\hline$C(55)-C(56)$ & $1.361(14)$ \\
\hline $\mathrm{C}(55)-\mathrm{H}(55)$ & 0.9500 \\
\hline$C(56)-C(57)$ & $1.382(14)$ \\
\hline $\mathrm{C}(56)-\mathrm{H}(56)$ & 0.9500 \\
\hline$C(57)-C(58)$ & $1.396(12)$ \\
\hline $\mathrm{C}(57)-\mathrm{H}(57)$ & 0.9500 \\
\hline $\mathrm{C}(58)-\mathrm{H}(58)$ & 0.9500 \\
\hline$C(59)-C(60)$ & $1.523(10)$ \\
\hline $\mathrm{C}(59)-\mathrm{H}(59 \mathrm{~A})$ & 0.9900 \\
\hline $\mathrm{C}(59)-\mathrm{H}(59 \mathrm{~B})$ & 0.9900 \\
\hline$C(60)-C(61)$ & $1.480(11)$ \\
\hline$C(61)-C(62)$ & $1.389(10)$ \\
\hline$C(61)-C(66)$ & $1.395(11)$ \\
\hline$C(62)-C(63)$ & $1.372(11)$ \\
\hline $\mathrm{C}(62)-\mathrm{H}(62)$ & 0.9500 \\
\hline$C(63)-C(64)$ & $1.404(10)$ \\
\hline $\mathrm{C}(63)-\mathrm{H}(63)$ & 0.9500 \\
\hline$C(64)-C(65)$ & $1.386(10)$ \\
\hline$C(64)-C(67)$ & $1.485(10)$ \\
\hline$C(65)-C(66)$ & $1.395(11)$ \\
\hline $\mathrm{C}(65)-\mathrm{H}(65)$ & 0.9500 \\
\hline $\mathrm{C}(66)-\mathrm{H}(66)$ & 0.9500 \\
\hline $\mathrm{C}(67)-\mathrm{C}(72)$ & $1.395(11)$ \\
\hline $\mathrm{C}(67)-\mathrm{C}(68)$ & $1.410(11)$ \\
\hline$C(68)-C(69)$ & $1.384(11)$ \\
\hline $\mathrm{C}(68)-\mathrm{H}(68)$ & 0.9500 \\
\hline$C(69)-C(70)$ & $1.385(12)$ \\
\hline $\mathrm{C}(69)-\mathrm{H}(69)$ & 0.9500 \\
\hline $\mathrm{C}(70)-\mathrm{C}(71)$ & $1.401(13)$ \\
\hline $\mathrm{C}(70)-\mathrm{H}(70)$ & 0.9500 \\
\hline $\mathrm{C}(71)-\mathrm{C}(72)$ & $1.391(12)$ \\
\hline $\mathrm{C}(71)-\mathrm{H}(71)$ & 0.9500 \\
\hline $\mathrm{C}(72)-\mathrm{H}(72)$ & 0.9500 \\
\hline $\mathrm{C}(5)-\mathrm{O}(1)-\mathrm{C}(8)$ & $117.4(7)$ \\
\hline $\mathrm{C}(41)-\mathrm{O}(4)-\mathrm{C}(44)$ & $115.9(7)$ \\
\hline$C(2)-C(1)-C(23)$ & $109.0(6)$ \\
\hline $\mathrm{C}(2)-\mathrm{C}(1)-\mathrm{C}(9)$ & $113.8(6)$ \\
\hline
\end{tabular}




\begin{tabular}{|c|c|}
\hline $\mathrm{C}(23)-\mathrm{C}(1)-\mathrm{C}(9)$ & $110.8(7)$ \\
\hline $\mathrm{C}(2)-\mathrm{C}(1)-\mathrm{H}(1)$ & 107.7 \\
\hline $\mathrm{C}(23)-\mathrm{C}(1)-\mathrm{H}(1)$ & 107.7 \\
\hline $\mathrm{C}(9)-\mathrm{C}(1)-\mathrm{H}(1)$ & 107.7 \\
\hline $\mathrm{C}(7)-\mathrm{C}(2)-\mathrm{C}(3)$ & $117.2(7)$ \\
\hline $\mathrm{C}(7)-\mathrm{C}(2)-\mathrm{C}(1)$ & $122.1(7)$ \\
\hline $\mathrm{C}(3)-\mathrm{C}(2)-\mathrm{C}(1)$ & $120.6(7)$ \\
\hline $\mathrm{C}(4)-\mathrm{C}(3)-\mathrm{C}(2)$ & $121.1(7)$ \\
\hline $\mathrm{C}(4)-\mathrm{C}(3)-\mathrm{H}(3)$ & 119.5 \\
\hline $\mathrm{C}(2)-\mathrm{C}(3)-\mathrm{H}(3)$ & 119.5 \\
\hline$C(5)-C(4)-C(3)$ & $119.6(7)$ \\
\hline $\mathrm{C}(5)-\mathrm{C}(4)-\mathrm{H}(4)$ & 120.2 \\
\hline $\mathrm{C}(3)-\mathrm{C}(4)-\mathrm{H}(4)$ & 120.2 \\
\hline $\mathrm{C}(4)-\mathrm{C}(5)-\mathrm{O}(1)$ & $124.5(7)$ \\
\hline$C(4)-C(5)-C(6)$ & $120.6(8)$ \\
\hline $\mathrm{O}(1)-\mathrm{C}(5)-\mathrm{C}(6)$ & $114.8(7)$ \\
\hline$C(7)-C(6)-C(5)$ & $118.4(7)$ \\
\hline $\mathrm{C}(7)-\mathrm{C}(6)-\mathrm{H}(6)$ & 120.8 \\
\hline $\mathrm{C}(5)-\mathrm{C}(6)-\mathrm{H}(6)$ & 120.8 \\
\hline $\mathrm{C}(6)-\mathrm{C}(7)-\mathrm{C}(2)$ & $123.1(7)$ \\
\hline $\mathrm{C}(6)-\mathrm{C}(7)-\mathrm{H}(7)$ & 118.5 \\
\hline $\mathrm{C}(2)-\mathrm{C}(7)-\mathrm{H}(7)$ & 118.5 \\
\hline $\mathrm{O}(1)-\mathrm{C}(8)-\mathrm{H}(8 \mathrm{~A})$ & 109.5 \\
\hline $\mathrm{O}(1)-\mathrm{C}(8)-\mathrm{H}(8 \mathrm{~B})$ & 109.5 \\
\hline $\mathrm{H}(8 \mathrm{~A})-\mathrm{C}(8)-\mathrm{H}(8 \mathrm{~B})$ & 109.5 \\
\hline $\mathrm{O}(1)-\mathrm{C}(8)-\mathrm{H}(8 \mathrm{C})$ & 109.5 \\
\hline $\mathrm{H}(8 \mathrm{~A})-\mathrm{C}(8)-\mathrm{H}(8 \mathrm{C})$ & 109.5 \\
\hline $\mathrm{H}(8 \mathrm{~B})-\mathrm{C}(8)-\mathrm{H}(8 \mathrm{C})$ & 109.5 \\
\hline $\mathrm{C}(10)-\mathrm{C}(9)-\mathrm{C}(1)$ & $113.5(6)$ \\
\hline $\mathrm{C}(10)-\mathrm{C}(9)-\mathrm{H}(9 \mathrm{~A})$ & 108.9 \\
\hline $\mathrm{C}(1)-\mathrm{C}(9)-\mathrm{H}(9 \mathrm{~A})$ & 108.9 \\
\hline $\mathrm{C}(10)-\mathrm{C}(9)-\mathrm{H}(9 \mathrm{AB})$ & 108.9 \\
\hline $\mathrm{C}(1)-\mathrm{C}(9)-\mathrm{H}(9 \mathrm{AB})$ & 108.9 \\
\hline $\mathrm{H}(9 \mathrm{~A})-\mathrm{C}(9)-\mathrm{H}(9 \mathrm{AB})$ & 107.7 \\
\hline $\mathrm{O}(2)-\mathrm{C}(10)-\mathrm{C}(11)$ & $119.9(6)$ \\
\hline $\mathrm{O}(2)-\mathrm{C}(10)-\mathrm{C}(9)$ & $121.0(6)$ \\
\hline $\mathrm{C}(11)-\mathrm{C}(10)-\mathrm{C}(9)$ & $119.2(6)$ \\
\hline $\mathrm{C}(16)-\mathrm{C}(11)-\mathrm{C}(12)$ & $118.2(7)$ \\
\hline
\end{tabular}




\begin{tabular}{|c|c|}
\hline $\mathrm{C}(16)-\mathrm{C}(11)-\mathrm{C}(10)$ & $118.6(7)$ \\
\hline $\mathrm{C}(12)-\mathrm{C}(11)-\mathrm{C}(10)$ & $123.1(7)$ \\
\hline $\mathrm{C}(13)-\mathrm{C}(12)-\mathrm{C}(11)$ & $120.9(7)$ \\
\hline $\mathrm{C}(13)-\mathrm{C}(12)-\mathrm{H}(12)$ & 119.5 \\
\hline $\mathrm{C}(11)-\mathrm{C}(12)-\mathrm{H}(12)$ & 119.5 \\
\hline $\mathrm{C}(12)-\mathrm{C}(13)-\mathrm{C}(14)$ & 121.1(7) \\
\hline $\mathrm{C}(12)-\mathrm{C}(13)-\mathrm{H}(13)$ & 119.4 \\
\hline $\mathrm{C}(14)-\mathrm{C}(13)-\mathrm{H}(13)$ & 119.4 \\
\hline $\mathrm{C}(15)-\mathrm{C}(14)-\mathrm{C}(13)$ & $117.9(7)$ \\
\hline $\mathrm{C}(15)-\mathrm{C}(14)-\mathrm{C}(17)$ & $121.3(7)$ \\
\hline $\mathrm{C}(13)-\mathrm{C}(14)-\mathrm{C}(17)$ & $120.8(7)$ \\
\hline $\mathrm{C}(14)-\mathrm{C}(15)-\mathrm{C}(16)$ & $120.9(7)$ \\
\hline $\mathrm{C}(14)-\mathrm{C}(15)-\mathrm{H}(15)$ & 119.6 \\
\hline $\mathrm{C}(16)-\mathrm{C}(15)-\mathrm{H}(15)$ & 119.6 \\
\hline $\mathrm{C}(11)-\mathrm{C}(16)-\mathrm{C}(15)$ & $120.9(7)$ \\
\hline $\mathrm{C}(11)-\mathrm{C}(16)-\mathrm{H}(16)$ & 119.6 \\
\hline $\mathrm{C}(15)-\mathrm{C}(16)-\mathrm{H}(16)$ & 119.6 \\
\hline $\mathrm{C}(18)-\mathrm{C}(17)-\mathrm{C}(22)$ & $118.8(7)$ \\
\hline $\mathrm{C}(18)-\mathrm{C}(17)-\mathrm{C}(14)$ & $120.9(7)$ \\
\hline $\mathrm{C}(22)-\mathrm{C}(17)-\mathrm{C}(14)$ & $120.3(7)$ \\
\hline $\mathrm{C}(19)-\mathrm{C}(18)-\mathrm{C}(17)$ & $120.4(8)$ \\
\hline $\mathrm{C}(19)-\mathrm{C}(18)-\mathrm{H}(18)$ & 119.8 \\
\hline $\mathrm{C}(17)-\mathrm{C}(18)-\mathrm{H}(18)$ & 119.8 \\
\hline $\mathrm{C}(18)-\mathrm{C}(19)-\mathrm{C}(20)$ & $120.8(8)$ \\
\hline $\mathrm{C}(18)-\mathrm{C}(19)-\mathrm{H}(19)$ & 119.6 \\
\hline $\mathrm{C}(20)-\mathrm{C}(19)-\mathrm{H}(19)$ & 119.6 \\
\hline $\mathrm{C}(21)-\mathrm{C}(20)-\mathrm{C}(19)$ & $119.3(8)$ \\
\hline $\mathrm{C}(21)-\mathrm{C}(20)-\mathrm{H}(20)$ & 120.4 \\
\hline $\mathrm{C}(19)-\mathrm{C}(20)-\mathrm{H}(20)$ & 120.4 \\
\hline$C(20)-C(21)-C(22)$ & $120.9(8)$ \\
\hline $\mathrm{C}(20)-\mathrm{C}(21)-\mathrm{H}(21)$ & 119.5 \\
\hline $\mathrm{C}(22)-\mathrm{C}(21)-\mathrm{H}(21)$ & 119.5 \\
\hline $\mathrm{C}(21)-\mathrm{C}(22)-\mathrm{C}(17)$ & $119.8(8)$ \\
\hline $\mathrm{C}(21)-\mathrm{C}(22)-\mathrm{H}(22)$ & 120.1 \\
\hline $\mathrm{C}(17)-\mathrm{C}(22)-\mathrm{H}(22)$ & 120.1 \\
\hline$C(24)-C(23)-C(1)$ & $115.3(6)$ \\
\hline $\mathrm{C}(24)-\mathrm{C}(23)-\mathrm{H}(23 \mathrm{~A})$ & 108.5 \\
\hline $\mathrm{C}(1)-\mathrm{C}(23)-\mathrm{H}(23 \mathrm{~A})$ & 108.5 \\
\hline
\end{tabular}




\begin{tabular}{|c|c|}
\hline $\mathrm{C}(24)-\mathrm{C}(23)-\mathrm{H}(23 \mathrm{~B})$ & 108.5 \\
\hline $\mathrm{C}(1)-\mathrm{C}(23)-\mathrm{H}(23 \mathrm{~B})$ & 108.5 \\
\hline $\mathrm{H}(23 \mathrm{~A})-\mathrm{C}(23)-\mathrm{H}(23 \mathrm{~B})$ & 107.5 \\
\hline $\mathrm{O}(3)-\mathrm{C}(24)-\mathrm{C}(25)$ & $120.6(8)$ \\
\hline $\mathrm{O}(3)-\mathrm{C}(24)-\mathrm{C}(23)$ & $121.7(7)$ \\
\hline$C(25)-C(24)-C(23)$ & $117.6(7)$ \\
\hline$C(26)-C(25)-C(30)$ & $118.9(7)$ \\
\hline$C(26)-C(25)-C(24)$ & $119.6(7)$ \\
\hline$C(30)-C(25)-C(24)$ & $121.5(7)$ \\
\hline$C(25)-C(26)-C(27)$ & $121.1(8)$ \\
\hline $\mathrm{C}(25)-\mathrm{C}(26)-\mathrm{H}(26)$ & 119.5 \\
\hline $\mathrm{C}(27)-\mathrm{C}(26)-\mathrm{H}(26)$ & 119.5 \\
\hline $\mathrm{C}(26)-\mathrm{C}(27)-\mathrm{C}(28)$ & $121.2(8)$ \\
\hline $\mathrm{C}(26)-\mathrm{C}(27)-\mathrm{H}(27)$ & 119.4 \\
\hline $\mathrm{C}(28)-\mathrm{C}(27)-\mathrm{H}(27)$ & 119.4 \\
\hline$C(29)-C(28)-C(27)$ & $116.7(7)$ \\
\hline $\mathrm{C}(29)-\mathrm{C}(28)-\mathrm{C}(31)$ & $120.7(7)$ \\
\hline $\mathrm{C}(27)-\mathrm{C}(28)-\mathrm{C}(31)$ & $122.5(7)$ \\
\hline $\mathrm{C}(30)-\mathrm{C}(29)-\mathrm{C}(28)$ & $121.6(7)$ \\
\hline $\mathrm{C}(30)-\mathrm{C}(29)-\mathrm{H}(29)$ & 119.2 \\
\hline $\mathrm{C}(28)-\mathrm{C}(29)-\mathrm{H}(29)$ & 119.2 \\
\hline$C(29)-C(30)-C(25)$ & $120.5(7)$ \\
\hline $\mathrm{C}(29)-\mathrm{C}(30)-\mathrm{H}(30)$ & 119.7 \\
\hline $\mathrm{C}(25)-\mathrm{C}(30)-\mathrm{H}(30)$ & 119.7 \\
\hline $\mathrm{C}(32)-\mathrm{C}(31)-\mathrm{C}(36)$ & $119.1(8)$ \\
\hline $\mathrm{C}(32)-\mathrm{C}(31)-\mathrm{C}(28)$ & $120.2(7)$ \\
\hline $\mathrm{C}(36)-\mathrm{C}(31)-\mathrm{C}(28)$ & $120.6(7)$ \\
\hline $\mathrm{C}(31)-\mathrm{C}(32)-\mathrm{C}(33)$ & $120.0(8)$ \\
\hline $\mathrm{C}(31)-\mathrm{C}(32)-\mathrm{H}(32)$ & 120.0 \\
\hline $\mathrm{C}(33)-\mathrm{C}(32)-\mathrm{H}(32)$ & 120.0 \\
\hline $\mathrm{C}(34)-\mathrm{C}(33)-\mathrm{C}(32)$ & $121.3(9)$ \\
\hline $\mathrm{C}(34)-\mathrm{C}(33)-\mathrm{H}(33)$ & 119.3 \\
\hline $\mathrm{C}(32)-\mathrm{C}(33)-\mathrm{H}(33)$ & 119.3 \\
\hline $\mathrm{C}(35)-\mathrm{C}(34)-\mathrm{C}(33)$ & $118.7(9)$ \\
\hline $\mathrm{C}(35)-\mathrm{C}(34)-\mathrm{H}(34)$ & 120.7 \\
\hline $\mathrm{C}(33)-\mathrm{C}(34)-\mathrm{H}(34)$ & 120.7 \\
\hline $\mathrm{C}(34)-\mathrm{C}(35)-\mathrm{C}(36)$ & $120.1(8)$ \\
\hline $\mathrm{C}(34)-\mathrm{C}(35)-\mathrm{H}(35)$ & 119.9 \\
\hline
\end{tabular}




\begin{tabular}{|c|c|}
\hline $\mathrm{C}(36)-\mathrm{C}(35)-\mathrm{H}(35)$ & 119.9 \\
\hline $\mathrm{C}(31)-\mathrm{C}(36)-\mathrm{C}(35)$ & $120.7(8)$ \\
\hline $\mathrm{C}(31)-\mathrm{C}(36)-\mathrm{H}(36)$ & 119.7 \\
\hline $\mathrm{C}(35)-\mathrm{C}(36)-\mathrm{H}(36)$ & 119.7 \\
\hline C(59)-C(37)-C(38) & $111.9(6)$ \\
\hline $\mathrm{C}(59)-\mathrm{C}(37)-\mathrm{C}(45)$ & $112.1(6)$ \\
\hline $\mathrm{C}(38)-\mathrm{C}(37)-\mathrm{C}(45)$ & $110.4(6)$ \\
\hline $\mathrm{C}(59)-\mathrm{C}(37)-\mathrm{H}(37)$ & 107.4 \\
\hline $\mathrm{C}(38)-\mathrm{C}(37)-\mathrm{H}(37)$ & 107.4 \\
\hline $\mathrm{C}(45)-\mathrm{C}(37)-\mathrm{H}(37)$ & 107.4 \\
\hline C(39)-C(38)-C(43) & $118.3(7)$ \\
\hline $\mathrm{C}(39)-\mathrm{C}(38)-\mathrm{C}(37)$ & $121.4(7)$ \\
\hline $\mathrm{C}(43)-\mathrm{C}(38)-\mathrm{C}(37)$ & $120.3(7)$ \\
\hline $\mathrm{C}(38)-\mathrm{C}(39)-\mathrm{C}(40)$ & $121.2(7)$ \\
\hline $\mathrm{C}(38)-\mathrm{C}(39)-\mathrm{H}(39)$ & 119.4 \\
\hline $\mathrm{C}(40)-\mathrm{C}(39)-\mathrm{H}(39)$ & 119.4 \\
\hline $\mathrm{C}(41)-\mathrm{C}(40)-\mathrm{C}(39)$ & $120.2(7)$ \\
\hline $\mathrm{C}(41)-\mathrm{C}(40)-\mathrm{H}(40)$ & 119.9 \\
\hline $\mathrm{C}(39)-\mathrm{C}(40)-\mathrm{H}(40)$ & 119.9 \\
\hline $\mathrm{O}(4)-\mathrm{C}(41)-\mathrm{C}(40)$ & $125.9(7)$ \\
\hline $\mathrm{O}(4)-\mathrm{C}(41)-\mathrm{C}(42)$ & $114.7(7)$ \\
\hline $\mathrm{C}(40)-\mathrm{C}(41)-\mathrm{C}(42)$ & $119.3(7)$ \\
\hline $\mathrm{C}(43)-\mathrm{C}(42)-\mathrm{C}(41)$ & $119.8(8)$ \\
\hline $\mathrm{C}(43)-\mathrm{C}(42)-\mathrm{H}(42)$ & 120.1 \\
\hline $\mathrm{C}(41)-\mathrm{C}(42)-\mathrm{H}(42)$ & 120.1 \\
\hline$C(38)-C(43)-C(42)$ & $121.0(7)$ \\
\hline $\mathrm{C}(38)-\mathrm{C}(43)-\mathrm{H}(43)$ & 119.5 \\
\hline $\mathrm{C}(42)-\mathrm{C}(43)-\mathrm{H}(43)$ & 119.5 \\
\hline $\mathrm{O}(4)-\mathrm{C}(44)-\mathrm{H}(44 \mathrm{~A})$ & 109.5 \\
\hline $\mathrm{O}(4)-\mathrm{C}(44)-\mathrm{H}(44 \mathrm{~B})$ & 109.5 \\
\hline $\mathrm{H}(44 \mathrm{~A})-\mathrm{C}(44)-\mathrm{H}(44 \mathrm{~B})$ & 109.5 \\
\hline $\mathrm{O}(4)-\mathrm{C}(44)-\mathrm{H}(44 \mathrm{C})$ & 109.5 \\
\hline $\mathrm{H}(44 \mathrm{~A})-\mathrm{C}(44)-\mathrm{H}(44 \mathrm{C})$ & 109.5 \\
\hline $\mathrm{H}(44 \mathrm{~B})-\mathrm{C}(44)-\mathrm{H}(44 \mathrm{C})$ & 109.5 \\
\hline$C(46)-C(45)-C(37)$ & $114.3(6)$ \\
\hline $\mathrm{C}(46)-\mathrm{C}(45)-\mathrm{H}(45 \mathrm{~A})$ & 108.7 \\
\hline $\mathrm{C}(37)-\mathrm{C}(45)-\mathrm{H}(45 \mathrm{~A})$ & 108.7 \\
\hline $\mathrm{C}(46)-\mathrm{C}(45)-\mathrm{H}(45 \mathrm{~B})$ & 108.7 \\
\hline
\end{tabular}




\begin{tabular}{|c|c|}
\hline $\mathrm{C}(37)-\mathrm{C}(45)-\mathrm{H}(45 \mathrm{~B})$ & 108.7 \\
\hline $\mathrm{H}(45 \mathrm{~A})-\mathrm{C}(45)-\mathrm{H}(45 \mathrm{~B})$ & 107.6 \\
\hline $\mathrm{O}(5)-\mathrm{C}(46)-\mathrm{C}(47)$ & $119.9(7)$ \\
\hline $\mathrm{O}(5)-\mathrm{C}(46)-\mathrm{C}(45)$ & $121.5(7)$ \\
\hline $\mathrm{C}(47)-\mathrm{C}(46)-\mathrm{C}(45)$ & $118.6(7)$ \\
\hline $\mathrm{C}(48)-\mathrm{C}(47)-\mathrm{C}(52)$ & $117.9(8)$ \\
\hline $\mathrm{C}(48)-\mathrm{C}(47)-\mathrm{C}(46)$ & $123.4(7)$ \\
\hline$C(52)-C(47)-C(46)$ & $118.7(7)$ \\
\hline C(49)-C(48)-C(47) & $121.6(8)$ \\
\hline $\mathrm{C}(49)-\mathrm{C}(48)-\mathrm{H}(48)$ & 119.2 \\
\hline $\mathrm{C}(47)-\mathrm{C}(48)-\mathrm{H}(48)$ & 119.2 \\
\hline $\mathrm{C}(48)-\mathrm{C}(49)-\mathrm{C}(50)$ & $121.5(8)$ \\
\hline $\mathrm{C}(48)-\mathrm{C}(49)-\mathrm{H}(49)$ & 119.3 \\
\hline $\mathrm{C}(50)-\mathrm{C}(49)-\mathrm{H}(49)$ & 119.3 \\
\hline $\mathrm{C}(49)-\mathrm{C}(50)-\mathrm{C}(51)$ & $117.5(8)$ \\
\hline $\mathrm{C}(49)-\mathrm{C}(50)-\mathrm{C}(53)$ & $122.2(7)$ \\
\hline $\mathrm{C}(51)-\mathrm{C}(50)-\mathrm{C}(53)$ & $120.3(7)$ \\
\hline $\mathrm{C}(52)-\mathrm{C}(51)-\mathrm{C}(50)$ & $120.8(7)$ \\
\hline $\mathrm{C}(52)-\mathrm{C}(51)-\mathrm{H}(51)$ & 119.6 \\
\hline $\mathrm{C}(50)-\mathrm{C}(51)-\mathrm{H}(51)$ & 119.6 \\
\hline $\mathrm{C}(47)-\mathrm{C}(52)-\mathrm{C}(51)$ & $120.8(7)$ \\
\hline $\mathrm{C}(47)-\mathrm{C}(52)-\mathrm{H}(52)$ & 119.6 \\
\hline $\mathrm{C}(51)-\mathrm{C}(52)-\mathrm{H}(52)$ & 119.6 \\
\hline $\mathrm{C}(54)-\mathrm{C}(53)-\mathrm{C}(58)$ & $117.8(8)$ \\
\hline $\mathrm{C}(54)-\mathrm{C}(53)-\mathrm{C}(50)$ & 121.2(7) \\
\hline $\mathrm{C}(58)-\mathrm{C}(53)-\mathrm{C}(50)$ & $120.9(8)$ \\
\hline $\mathrm{C}(53)-\mathrm{C}(54)-\mathrm{C}(55)$ & 121.1(8) \\
\hline $\mathrm{C}(53)-\mathrm{C}(54)-\mathrm{H}(54)$ & 119.4 \\
\hline $\mathrm{C}(55)-\mathrm{C}(54)-\mathrm{H}(54)$ & 119.4 \\
\hline $\mathrm{C}(56)-\mathrm{C}(55)-\mathrm{C}(54)$ & $120.0(9)$ \\
\hline $\mathrm{C}(56)-\mathrm{C}(55)-\mathrm{H}(55)$ & 120.0 \\
\hline $\mathrm{C}(54)-\mathrm{C}(55)-\mathrm{H}(55)$ & 120.0 \\
\hline $\mathrm{C}(55)-\mathrm{C}(56)-\mathrm{C}(57)$ & $121.0(9)$ \\
\hline $\mathrm{C}(55)-\mathrm{C}(56)-\mathrm{H}(56)$ & 119.5 \\
\hline $\mathrm{C}(57)-\mathrm{C}(56)-\mathrm{H}(56)$ & 119.5 \\
\hline $\mathrm{C}(56)-\mathrm{C}(57)-\mathrm{C}(58)$ & $119.0(9)$ \\
\hline $\mathrm{C}(56)-\mathrm{C}(57)-\mathrm{H}(57)$ & 120.5 \\
\hline $\mathrm{C}(58)-\mathrm{C}(57)-\mathrm{H}(57)$ & 120.5 \\
\hline
\end{tabular}




\begin{tabular}{|c|c|}
\hline $\mathrm{C}(57)-\mathrm{C}(58)-\mathrm{C}(53)$ & $121.0(8)$ \\
\hline $\mathrm{C}(57)-\mathrm{C}(58)-\mathrm{H}(58)$ & 119.5 \\
\hline $\mathrm{C}(53)-\mathrm{C}(58)-\mathrm{H}(58)$ & 119.5 \\
\hline $\mathrm{C}(37)-\mathrm{C}(59)-\mathrm{C}(60)$ & $113.3(6)$ \\
\hline $\mathrm{C}(37)-\mathrm{C}(59)-\mathrm{H}(59 \mathrm{~A})$ & 108.9 \\
\hline $\mathrm{C}(60)-\mathrm{C}(59)-\mathrm{H}(59 \mathrm{~A})$ & 108.9 \\
\hline $\mathrm{C}(37)-\mathrm{C}(59)-\mathrm{H}(59 \mathrm{~B})$ & 108.9 \\
\hline $\mathrm{C}(60)-\mathrm{C}(59)-\mathrm{H}(59 \mathrm{~B})$ & 108.9 \\
\hline $\mathrm{H}(59 \mathrm{~A})-\mathrm{C}(59)-\mathrm{H}(59 \mathrm{~B})$ & 107.7 \\
\hline $\mathrm{O}(6)-\mathrm{C}(60)-\mathrm{C}(61)$ & $119.7(7)$ \\
\hline $\mathrm{O}(6)-\mathrm{C}(60)-\mathrm{C}(59)$ & $120.3(7)$ \\
\hline$C(61)-C(60)-C(59)$ & $119.9(6)$ \\
\hline$C(62)-C(61)-C(66)$ & $119.0(7)$ \\
\hline$C(62)-C(61)-C(60)$ & $123.1(7)$ \\
\hline$C(66)-C(61)-C(60)$ & $117.8(7)$ \\
\hline $\mathrm{C}(63)-\mathrm{C}(62)-\mathrm{C}(61)$ & $121.3(7)$ \\
\hline $\mathrm{C}(63)-\mathrm{C}(62)-\mathrm{H}(62)$ & 119.4 \\
\hline $\mathrm{C}(61)-\mathrm{C}(62)-\mathrm{H}(62)$ & 119.4 \\
\hline$C(62)-C(63)-C(64)$ & $120.0(7)$ \\
\hline $\mathrm{C}(62)-\mathrm{C}(63)-\mathrm{H}(63)$ & 120.0 \\
\hline $\mathrm{C}(64)-\mathrm{C}(63)-\mathrm{H}(63)$ & 120.0 \\
\hline$C(65)-C(64)-C(63)$ & $119.3(7)$ \\
\hline$C(65)-C(64)-C(67)$ & $120.2(7)$ \\
\hline$C(63)-C(64)-C(67)$ & $120.5(7)$ \\
\hline$C(64)-C(65)-C(66)$ & $120.3(7)$ \\
\hline $\mathrm{C}(64)-\mathrm{C}(65)-\mathrm{H}(65)$ & 119.8 \\
\hline $\mathrm{C}(66)-\mathrm{C}(65)-\mathrm{H}(65)$ & 119.8 \\
\hline$C(61)-C(66)-C(65)$ & $120.1(7)$ \\
\hline $\mathrm{C}(61)-\mathrm{C}(66)-\mathrm{H}(66)$ & 119.9 \\
\hline $\mathrm{C}(65)-\mathrm{C}(66)-\mathrm{H}(66)$ & 119.9 \\
\hline$C(72)-C(67)-C(68)$ & $118.1(7)$ \\
\hline$C(72)-C(67)-C(64)$ & $121.2(7)$ \\
\hline$C(68)-C(67)-C(64)$ & $120.7(7)$ \\
\hline$C(69)-C(68)-C(67)$ & $120.1(8)$ \\
\hline $\mathrm{C}(69)-\mathrm{C}(68)-\mathrm{H}(68)$ & 119.9 \\
\hline $\mathrm{C}(67)-\mathrm{C}(68)-\mathrm{H}(68)$ & 119.9 \\
\hline $\mathrm{C}(68)-\mathrm{C}(69)-\mathrm{C}(70)$ & $121.4(8)$ \\
\hline $\mathrm{C}(68)-\mathrm{C}(69)-\mathrm{H}(69)$ & 119.3 \\
\hline
\end{tabular}




$\begin{array}{ll}\mathrm{C}(70)-\mathrm{C}(69)-\mathrm{H}(69) & 119.3 \\ \mathrm{C}(69)-\mathrm{C}(70)-\mathrm{C}(71) & 119.2(8) \\ \mathrm{C}(69)-\mathrm{C}(70)-\mathrm{H}(70) & 120.4 \\ \mathrm{C}(71)-\mathrm{C}(70)-\mathrm{H}(70) & 120.4 \\ \mathrm{C}(72)-\mathrm{C}(71)-\mathrm{C}(70) & 119.5(8) \\ \mathrm{C}(72)-\mathrm{C}(71)-\mathrm{H}(71) & 120.2 \\ \mathrm{C}(70)-\mathrm{C}(71)-\mathrm{H}(71) & 120.2 \\ \mathrm{C}(71)-\mathrm{C}(72)-\mathrm{C}(67) & 121.6(8) \\ \mathrm{C}(71)-\mathrm{C}(72)-\mathrm{H}(72) & 119.2 \\ \mathrm{C}(67)-\mathrm{C}(72)-\mathrm{H}(72) & 119.2\end{array}$

Symmetry transformations used to generate equivalent atoms: 
Table S16. Anisotropic displacement parameters $\left(\AA^{2} \times 10^{3}\right)$ for i17966. The anisotropic displacement factor exponent takes the form: $-2 \pi^{2}\left[h^{2} a^{* 2} U^{11}+\ldots+2 h k^{*} b^{*} U^{12}\right]$

\begin{tabular}{|c|c|c|c|c|c|c|}
\hline & $\mathrm{U}^{11}$ & $\mathrm{U}^{22}$ & $\mathrm{U}^{33}$ & $\mathrm{U}^{23}$ & $\mathrm{U}^{13}$ & $\mathrm{U}^{12}$ \\
\hline $\mathrm{O}(1)$ & $40(4)$ & $32(3)$ & $42(4)$ & $2(3)$ & $3(3)$ & $-6(3)$ \\
\hline $\mathrm{O}(2)$ & $22(3)$ & $35(3)$ & $24(3)$ & $6(2)$ & $-3(2)$ & $-8(2)$ \\
\hline $\mathrm{O}(3)$ & $30(3)$ & $36(3)$ & $30(3)$ & $8(2)$ & $-2(2)$ & $-6(3)$ \\
\hline $\mathrm{O}(4)$ & $39(3)$ & $22(3)$ & $45(4)$ & 11(3) & $-6(3)$ & $-4(3)$ \\
\hline $\mathrm{O}(5)$ & $30(3)$ & $30(3)$ & $33(3)$ & $-1(2)$ & $-3(2)$ & $-1(2)$ \\
\hline $\mathrm{O}(6)$ & $20(3)$ & $37(3)$ & $35(3)$ & $-6(3)$ & $6(2)$ & $-13(2)$ \\
\hline $\mathrm{C}(1)$ & $17(4)$ & $32(4)$ & $28(4)$ & $8(3)$ & $2(3)$ & $1(3)$ \\
\hline $\mathrm{C}(2)$ & $14(3)$ & $33(4)$ & $17(3)$ & $2(3)$ & $0(3)$ & $-7(3)$ \\
\hline $\mathrm{C}(3)$ & $21(4)$ & $34(4)$ & $32(4)$ & $6(3)$ & $1(3)$ & $-9(3)$ \\
\hline$C(4)$ & $24(4)$ & $33(4)$ & $26(4)$ & $1(3)$ & $5(3)$ & $-1(3)$ \\
\hline$C(5)$ & $33(4)$ & $31(4)$ & $16(3)$ & $3(3)$ & $2(3)$ & $-8(3)$ \\
\hline $\mathrm{C}(6)$ & $28(4)$ & $37(5)$ & $21(4)$ & $4(3)$ & $-1(3)$ & $-12(3)$ \\
\hline $\mathrm{C}(7)$ & $17(3)$ & $33(4)$ & 21(4) & $4(3)$ & $-1(3)$ & $-6(3)$ \\
\hline $\mathrm{C}(8)$ & $48(6)$ & $35(5)$ & $86(9)$ & $3(5)$ & 19(6) & $-3(5)$ \\
\hline $\mathrm{C}(9)$ & $25(4)$ & $34(4)$ & $21(4)$ & $3(3)$ & $0(3)$ & $-10(3)$ \\
\hline$C(10)$ & $16(3)$ & $24(4)$ & 20(3) & $2(3)$ & $-3(3)$ & $-2(3)$ \\
\hline $\mathrm{C}(11)$ & 21(4) & 21(4) & 21(4) & $2(3)$ & $1(3)$ & $-4(3)$ \\
\hline$C(12)$ & $24(4)$ & $28(4)$ & $24(4)$ & $3(3)$ & $-7(3)$ & $-7(3)$ \\
\hline $\mathrm{C}(13)$ & $21(4)$ & $34(4)$ & $22(4)$ & $-3(3)$ & $-2(3)$ & $-10(3)$ \\
\hline$C(14)$ & $24(4)$ & $25(4)$ & $19(3)$ & $3(3)$ & $-3(3)$ & $-5(3)$ \\
\hline$C(15)$ & $27(4)$ & $39(5)$ & $21(4)$ & $9(3)$ & $-8(3)$ & $-14(3)$ \\
\hline$C(16)$ & $22(4)$ & $44(5)$ & $30(4)$ & $9(4)$ & $-7(3)$ & $-11(4)$ \\
\hline$C(17)$ & $33(4)$ & $19(4)$ & $19(3)$ & $0(3)$ & $-3(3)$ & $-5(3)$ \\
\hline$C(18)$ & $26(4)$ & $29(4)$ & $26(4)$ & $-3(3)$ & $-5(3)$ & $-7(3)$ \\
\hline $\mathrm{C}(19)$ & $32(4)$ & $34(4)$ & $31(4)$ & $-9(3)$ & $6(3)$ & $-9(4)$ \\
\hline $\mathrm{C}(20)$ & $50(5)$ & $42(5)$ & $16(4)$ & $-2(3)$ & $2(3)$ & $-26(4)$ \\
\hline$C(21)$ & $35(5)$ & $37(5)$ & $31(4)$ & $7(4)$ & $-7(4)$ & $-10(4)$ \\
\hline $\mathrm{C}(22)$ & $30(4)$ & $31(4)$ & $24(4)$ & $-1(3)$ & $-2(3)$ & $-3(3)$ \\
\hline$C(23)$ & $24(4)$ & $29(4)$ & 21(4) & $4(3)$ & $5(3)$ & $-3(3)$ \\
\hline$C(24)$ & $25(4)$ & $31(4)$ & $23(4)$ & $6(3)$ & $1(3)$ & $-10(3)$ \\
\hline$C(25)$ & $26(4)$ & $33(4)$ & $21(4)$ & $2(3)$ & $3(3)$ & $-6(3)$ \\
\hline$C(26)$ & $37(5)$ & $33(4)$ & $23(4)$ & $7(3)$ & $-3(3)$ & $-2(4)$ \\
\hline \multirow[t]{2}{*}{$C(27)$} & $36(4)$ & $24(4)$ & $26(4)$ & $1(3)$ & $6(3)$ & $-1(3)$ \\
\hline & & & & S-38 & & \\
\hline
\end{tabular}




\begin{tabular}{|c|c|c|c|c|c|c|}
\hline$C(28)$ & $23(4)$ & $27(4)$ & $22(4)$ & $5(3)$ & 1(3) & $-5(3)$ \\
\hline$C(29)$ & $26(4)$ & $25(4)$ & $30(4)$ & $6(3)$ & $3(3)$ & $-6(3)$ \\
\hline$C(30)$ & $28(4)$ & $19(4)$ & $26(4)$ & $2(3)$ & $5(3)$ & 1(3) \\
\hline $\mathrm{C}(31)$ & $24(4)$ & $27(4)$ & $25(4)$ & $2(3)$ & $3(3)$ & $-1(3)$ \\
\hline$C(32)$ & $52(5)$ & $24(4)$ & $26(4)$ & $1(3)$ & $-2(4)$ & $-7(4)$ \\
\hline $\mathrm{C}(33)$ & $52(6)$ & $28(4)$ & $37(5)$ & $-5(4)$ & $-9(4)$ & $-5(4)$ \\
\hline $\mathrm{C}(34)$ & $43(5)$ & $36(5)$ & $36(5)$ & $-9(4)$ & $2(4)$ & $-7(4)$ \\
\hline$C(35)$ & $33(4)$ & $40(5)$ & $23(4)$ & $2(3)$ & $5(3)$ & $-8(4)$ \\
\hline$C(36)$ & $33(4)$ & $24(4)$ & $24(4)$ & $1(3)$ & $5(3)$ & $-1(3)$ \\
\hline $\mathrm{C}(37)$ & $16(3)$ & $26(4)$ & $27(4)$ & $-1(3)$ & $-2(3)$ & $-6(3)$ \\
\hline $\mathrm{C}(38)$ & $19(3)$ & $28(4)$ & $21(3)$ & $-2(3)$ & $7(3)$ & $-5(3)$ \\
\hline C(39) & $22(4)$ & $24(4)$ & $24(4)$ & $2(3)$ & $-6(3)$ & $-5(3)$ \\
\hline $\mathrm{C}(40)$ & $23(4)$ & $26(4)$ & $24(4)$ & $-1(3)$ & $-5(3)$ & $-5(3)$ \\
\hline $\mathrm{C}(41)$ & $25(4)$ & $23(4)$ & 21(4) & $1(3)$ & $-2(3)$ & $-6(3)$ \\
\hline $\mathrm{C}(42)$ & $26(4)$ & $31(4)$ & $28(4)$ & $-2(3)$ & $3(3)$ & $-6(3)$ \\
\hline$C(43)$ & $20(4)$ & $33(4)$ & $27(4)$ & $-3(3)$ & $4(3)$ & $-6(3)$ \\
\hline $\mathrm{C}(44)$ & $47(6)$ & $32(5)$ & $74(8)$ & $9(5)$ & $5(5)$ & $6(4)$ \\
\hline$C(45)$ & $24(4)$ & $22(4)$ & $28(4)$ & $0(3)$ & $-5(3)$ & $-5(3)$ \\
\hline$C(46)$ & $24(4)$ & $24(4)$ & $32(4)$ & $-5(3)$ & $-5(3)$ & $-7(3)$ \\
\hline $\mathrm{C}(47)$ & $20(4)$ & $21(4)$ & $33(4)$ & $0(3)$ & $-8(3)$ & $0(3)$ \\
\hline $\mathrm{C}(48)$ & $31(4)$ & $25(4)$ & $27(4)$ & $-5(3)$ & $-3(3)$ & 1(3) \\
\hline$C(49)$ & $32(4)$ & $23(4)$ & $32(4)$ & $-4(3)$ & $-1(3)$ & $-5(3)$ \\
\hline $\mathrm{C}(50)$ & $25(4)$ & $25(4)$ & $27(4)$ & $-5(3)$ & $-6(3)$ & $-6(3)$ \\
\hline $\mathrm{C}(51)$ & $38(5)$ & $22(4)$ & $27(4)$ & $3(3)$ & $-11(3)$ & $-3(3)$ \\
\hline$C(52)$ & $32(4)$ & $25(4)$ & $26(4)$ & $-6(3)$ & $-6(3)$ & $0(3)$ \\
\hline$C(53)$ & $33(4)$ & $25(4)$ & $31(4)$ & $-1(3)$ & $-5(3)$ & $-6(3)$ \\
\hline$C(54)$ & $33(4)$ & $30(4)$ & $30(4)$ & $1(3)$ & $2(3)$ & $-1(3)$ \\
\hline $\mathrm{C}(55)$ & $52(6)$ & $40(5)$ & $31(5)$ & $-6(4)$ & $12(4)$ & $-20(4)$ \\
\hline$C(56)$ & $49(6)$ & $47(5)$ & $27(4)$ & $0(4)$ & $-5(4)$ & $-23(5)$ \\
\hline $\mathrm{C}(57)$ & $50(6)$ & $28(4)$ & $34(5)$ & $3(4)$ & $-9(4)$ & $-15(4)$ \\
\hline $\mathrm{C}(58)$ & $37(5)$ & $31(4)$ & $26(4)$ & $3(3)$ & $-6(3)$ & $-6(4)$ \\
\hline C(59) & $26(4)$ & $29(4)$ & $26(4)$ & $0(3)$ & $4(3)$ & $-10(3)$ \\
\hline$C(60)$ & $20(4)$ & $20(4)$ & $28(4)$ & $-1(3)$ & $0(3)$ & $-4(3)$ \\
\hline $\mathrm{C}(61)$ & $21(4)$ & 19(3) & $27(4)$ & $2(3)$ & $3(3)$ & $-4(3)$ \\
\hline $\mathrm{C}(62)$ & $24(4)$ & 21(4) & $24(4)$ & $0(3)$ & $6(3)$ & $-5(3)$ \\
\hline $\mathrm{C}(63)$ & $24(4)$ & $26(4)$ & $31(4)$ & $1(3)$ & $5(3)$ & $-7(3)$ \\
\hline$C(64)$ & $19(3)$ & 21(4) & 21(3) & $4(3)$ & $-2(3)$ & $-1(3)$ \\
\hline$C(65)$ & $18(4)$ & $32(4)$ & $26(4)$ & $4(3)$ & $1(3)$ & $-7(3)$ \\
\hline
\end{tabular}




$\begin{array}{lllllll}\mathrm{C}(66) & 21(4) & 34(4) & 28(4) & 4(3) & -6(3) & -8(3) \\ \mathrm{C}(67) & 22(4) & 28(4) & 21(4) & 2(3) & 2(3) & -7(3) \\ \mathrm{C}(68) & 28(4) & 37(4) & 20(4) & 6(3) & 0(3) & -10(3) \\ \mathrm{C}(69) & 29(4) & 42(5) & 26(4) & 2(3) & -2(3) & -13(4) \\ \mathrm{C}(70) & 43(5) & 30(4) & 23(4) & 8(3) & -5(3) & -10(4) \\ \mathrm{C}(71) & 36(5) & 43(5) & 25(4) & -2(4) & 6(3) & -6(4) \\ \mathrm{C}(72) & 26(4) & 38(5) & 22(4) & -2(3) & 1(3) & -9(3)\end{array}$

\section{Crystal data and structure refinement for compound $\mathbf{4 r}$}

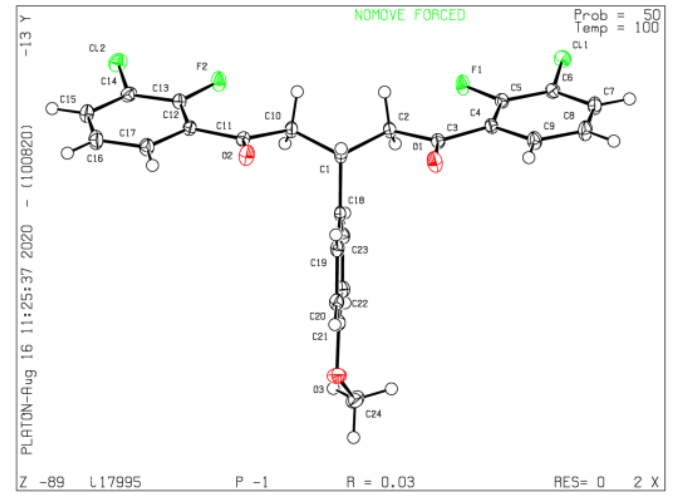

Figure S5. Single X-ray crystal structure of 1, 5-dicarbonyls 4r (the thermal ellipsoid was drawn at the 50\% probability level)

Table S17. Crystal data and structure refinement for i17995

\section{CCDC}

Identification code

Empirical formula

Formula weight

Temperature

Wavelength

Crystal system

Space group

Unit cell dimensions

Z, Volume

Density (calculated)

Absorption coefficient

$\mathrm{F}(000)$

Crystal size

Theta range for data collection

Index ranges
2031245

i17995

C24 H18 C12 F2 O3

463.28

100.0(2) K

$0.71073 \AA$

Triclinic

$\mathrm{P}-1$

$\mathrm{a}=8.9911(2) \AA$

$\alpha=88.9720(10)^{\circ}$.

$\mathrm{b}=10.8950(3) \AA$

$\beta=73.5650(10)^{\circ}$.

$\mathrm{c}=11.8009(3) \AA$

$\gamma=66.1240(10)^{\circ}$.

$2,1007.71(4) \AA^{3}$

$1.527 \mathrm{Mg} / \mathrm{m}^{3}$

$0.366 \mathrm{~mm}^{-1}$

476

$0.288 \times 0.227 \times 0.182 \mathrm{~mm}^{3}$

1.810 to $30.508^{\circ}$.

$-12<=\mathrm{h}<=12,-15<=\mathrm{k}<=15,-16<=\mathrm{l}<=16$ 
Reflections collected

Independent reflections

Completeness to theta $=25.242^{\circ}$

Absorption correction

Max. and min. transmission

Refinement method

Data / restraints / parameters

Goodness-of-fit on $\mathrm{F}^{2}$

Final $\mathrm{R}$ indices [I $>2 \operatorname{sigma}(\mathrm{I})]$

$\mathrm{R}$ indices (all data)

Extinction coefficient

Largest diff. peak and hole
52936

$6134[\mathrm{R}(\mathrm{int})=0.0380]$

$99.7 \%$

Numerical

1 and 0.9675

Full-matrix least-squares on $\mathrm{F}^{2}$

6134 / 0 / 281

1.022

$\mathrm{R} 1=0.0330, \mathrm{wR} 2=0.0811$

$\mathrm{R} 1=0.0405, \mathrm{wR} 2=0.0860$

$\mathrm{n} / \mathrm{a}$

0.442 and -0.372 e. $\AA^{-3}$

Table S18. Atomic coordinates ( $x \mathbf{1 0}^{4}$ ) and equivalent isotropic displacement parameters $\left(\AA^{2} \times 1^{3}\right)$ for i17995. $U(\mathrm{eq})$ is defined as one third of the trace of the orthogonalized $U^{i j}$ tensor.

\begin{tabular}{|c|c|c|c|c|}
\hline & $\mathrm{X}$ & $\mathrm{y}$ & $\mathrm{Z}$ & $\mathrm{U}(\mathrm{eq})$ \\
\hline $\mathrm{Cl}(1)$ & $12288(1)$ & $-1735(1)$ & $739(1)$ & $19(1)$ \\
\hline $\mathrm{Cl}(2)$ & $6259(1)$ & $9717(1)$ & $978(1)$ & $23(1)$ \\
\hline $\mathrm{F}(1)$ & $9606(1)$ & $900(1)$ & $1640(1)$ & $20(1)$ \\
\hline $\mathrm{F}(2)$ & $6384(1)$ & $7197(1)$ & $1880(1)$ & $21(1)$ \\
\hline $\mathrm{O}(1)$ & $5078(1)$ & $1829(1)$ & $4383(1)$ & $22(1)$ \\
\hline $\mathrm{O}(2)$ & $2673(1)$ & $6687(1)$ & $4619(1)$ & $21(1)$ \\
\hline $\mathrm{O}(3)$ & $-460(1)$ & 3998(1) & $1613(1)$ & $21(1)$ \\
\hline $\mathrm{C}(1)$ & $4790(1)$ & $4156(1)$ & $3337(1)$ & $14(1)$ \\
\hline$C(2)$ & $6453(1)$ & $2867(1)$ & 2914(1) & $15(1)$ \\
\hline$C(3)$ & $6318(1)$ & $1678(1)$ & $3544(1)$ & $15(1)$ \\
\hline$C(4)$ & $7724(1)$ & $278(1)$ & $3149(1)$ & $14(1)$ \\
\hline$C(5)$ & $9239(1)$ & $-61(1)$ & $2228(1)$ & $15(1)$ \\
\hline$C(6)$ & $10427(1)$ & $-1393(1)$ & $1884(1)$ & $15(1)$ \\
\hline$C(7)$ & $10132(2)$ & $-2428(1)$ & $2463(1)$ & $19(1)$ \\
\hline $\mathrm{C}(8)$ & $8634(2)$ & $-2115(1)$ & $3387(1)$ & $21(1)$ \\
\hline $\mathrm{C}(9)$ & $7457(2)$ & $-787(1)$ & $3722(1)$ & $18(1)$ \\
\hline$C(10)$ & $5133(1)$ & $5408(1)$ & $3000(1)$ & $16(1)$ \\
\hline $\mathrm{C}(11)$ & $3735(1)$ & $6705(1)$ & $3718(1)$ & $15(1)$ \\
\hline$C(12)$ & $3640(1)$ & $8049(1)$ & $3321(1)$ & $14(1)$ \\
\hline$C(13)$ & 4901(1) & $8248(1)$ & $2441(1)$ & $15(1)$ \\
\hline$C(14)$ & $4684(2)$ & $9524(1)$ & $2102(1)$ & $17(1)$ \\
\hline
\end{tabular}




\begin{tabular}{lrrrr}
$\mathrm{C}(15)$ & $3197(2)$ & $10639(1)$ & $2659(1)$ & $21(1)$ \\
$\mathrm{C}(16)$ & $1924(2)$ & $10472(1)$ & $3546(1)$ & $22(1)$ \\
$\mathrm{C}(17)$ & $2147(2)$ & $9193(1)$ & $3867(1)$ & $18(1)$ \\
$\mathrm{C}(18)$ & $3418(1)$ & $4100(1)$ & $2855(1)$ & $14(1)$ \\
$\mathrm{C}(19)$ & $1845(1)$ & $4223(1)$ & $3614(1)$ & $15(1)$ \\
$\mathrm{C}(20)$ & $584(1)$ & $4176(1)$ & $3181(1)$ & $16(1)$ \\
$\mathrm{C}(21)$ & $871(1)$ & $4000(1)$ & $1955(1)$ & $16(1)$ \\
$\mathrm{C}(22)$ & $2440(2)$ & $3852(1)$ & $1173(1)$ & $16(1)$ \\
$\mathrm{C}(23)$ & $3678(1)$ & $3914(1)$ & $1634(1)$ & $16(1)$ \\
$\mathrm{C}(24)$ & $-85(2)$ & $3408(1)$ & $438(1)$ & $23(1)$ \\
& & & & \\
\hline
\end{tabular}


Table S19. Bond lengths $[\AA]$ and angles $\left[{ }^{\circ}\right]$ for $\mathbf{i 1 7 9 9 5}$.

\begin{tabular}{|c|c|}
\hline $\mathrm{Cl}(1)-\mathrm{C}(6)$ & $1.7319(12)$ \\
\hline $\mathrm{Cl}(2)-\mathrm{C}(14)$ & $1.7253(12)$ \\
\hline$F(1)-C(5)$ & $1.3461(13)$ \\
\hline $\mathrm{F}(2)-\mathrm{C}(13)$ & $1.3489(13)$ \\
\hline $\mathrm{O}(1)-\mathrm{C}(3)$ & $1.2174(14)$ \\
\hline $\mathrm{O}(2)-\mathrm{C}(11)$ & $1.2182(14)$ \\
\hline $\mathrm{O}(3)-\mathrm{C}(21)$ & $1.3684(14)$ \\
\hline $\mathrm{O}(3)-\mathrm{C}(24)$ & $1.4280(15)$ \\
\hline $\mathrm{C}(1)-\mathrm{C}(18)$ & $1.5199(16)$ \\
\hline $\mathrm{C}(1)-\mathrm{C}(2)$ & $1.5366(15)$ \\
\hline $\mathrm{C}(1)-\mathrm{C}(10)$ & $1.5377(15)$ \\
\hline $\mathrm{C}(1)-\mathrm{H}(1)$ & 1.0000 \\
\hline$C(2)-C(3)$ & $1.5122(15)$ \\
\hline $\mathrm{C}(2)-\mathrm{H}(2 \mathrm{~A})$ & 0.9900 \\
\hline $\mathrm{C}(2)-\mathrm{H}(2 \mathrm{AB})$ & 0.9900 \\
\hline$C(3)-C(4)$ & $1.5081(15)$ \\
\hline $\mathrm{C}(4)-\mathrm{C}(5)$ & $1.3933(15)$ \\
\hline$C(4)-C(9)$ & $1.4024(15)$ \\
\hline $\mathrm{C}(5)-\mathrm{C}(6)$ & $1.3904(15)$ \\
\hline$C(6)-C(7)$ & $1.3856(16)$ \\
\hline$C(7)-C(8)$ & $1.3890(17)$ \\
\hline $\mathrm{C}(7)-\mathrm{H}(7)$ & 0.9500 \\
\hline $\mathrm{C}(8)-\mathrm{C}(9)$ & $1.3843(17)$ \\
\hline $\mathrm{C}(8)-\mathrm{H}(8)$ & 0.9500 \\
\hline $\mathrm{C}(9)-\mathrm{H}(9)$ & 0.9500 \\
\hline$C(10)-C(11)$ & $1.5163(15)$ \\
\hline $\mathrm{C}(10)-\mathrm{H}(10 \mathrm{~A})$ & 0.9900 \\
\hline $\mathrm{C}(10)-\mathrm{H}(10 \mathrm{~B})$ & 0.9900 \\
\hline$C(11)-C(12)$ & $1.5057(15)$ \\
\hline$C(12)-C(13)$ & $1.3913(16)$ \\
\hline$C(12)-C(17)$ & $1.3982(16)$ \\
\hline $\mathrm{C}(13)-\mathrm{C}(14)$ & $1.3912(16)$ \\
\hline$C(14)-C(15)$ & $1.3829(17)$ \\
\hline$C(15)-C(16)$ & $1.3863(18)$ \\
\hline $\mathrm{C}(15)-\mathrm{H}(15)$ & 0.9500 \\
\hline$C(16)-C(17)$ & $1.3870(17)$ \\
\hline
\end{tabular}




\begin{tabular}{|c|c|}
\hline $\mathrm{C}(16)-\mathrm{H}(16)$ & 0.9500 \\
\hline $\mathrm{C}(17)-\mathrm{H}(17)$ & 0.9500 \\
\hline $\mathrm{C}(18)-\mathrm{C}(23)$ & $1.3972(16)$ \\
\hline $\mathrm{C}(18)-\mathrm{C}(19)$ & $1.3977(15)$ \\
\hline$C(19)-C(20)$ & $1.3883(16)$ \\
\hline $\mathrm{C}(19)-\mathrm{H}(19)$ & 0.9500 \\
\hline $\mathrm{C}(20)-\mathrm{C}(21)$ & $1.3974(16)$ \\
\hline $\mathrm{C}(20)-\mathrm{H}(20)$ & 0.9500 \\
\hline $\mathrm{C}(21)-\mathrm{C}(22)$ & $1.3977(16)$ \\
\hline $\mathrm{C}(22)-\mathrm{C}(23)$ & $1.3954(16)$ \\
\hline $\mathrm{C}(22)-\mathrm{H}(22)$ & 0.9500 \\
\hline $\mathrm{C}(23)-\mathrm{H}(23)$ & 0.9500 \\
\hline $\mathrm{C}(24)-\mathrm{H}(24 \mathrm{~A})$ & 0.9800 \\
\hline C(24)-H(24B) & 0.9800 \\
\hline $\mathrm{C}(24)-\mathrm{H}(24 \mathrm{C})$ & 0.9800 \\
\hline $\mathrm{C}(21)-\mathrm{O}(3)-\mathrm{C}(24)$ & $117.57(10)$ \\
\hline $\mathrm{C}(18)-\mathrm{C}(1)-\mathrm{C}(2)$ & 111.66(9) \\
\hline C(18)-C(1)-C(10) & $111.35(9)$ \\
\hline $\mathrm{C}(2)-\mathrm{C}(1)-\mathrm{C}(10)$ & $110.30(9)$ \\
\hline $\mathrm{C}(18)-\mathrm{C}(1)-\mathrm{H}(1)$ & 107.8 \\
\hline $\mathrm{C}(2)-\mathrm{C}(1)-\mathrm{H}(1)$ & 107.8 \\
\hline $\mathrm{C}(10)-\mathrm{C}(1)-\mathrm{H}(1)$ & 107.8 \\
\hline $\mathrm{C}(3)-\mathrm{C}(2)-\mathrm{C}(1)$ & $112.16(9)$ \\
\hline $\mathrm{C}(3)-\mathrm{C}(2)-\mathrm{H}(2 \mathrm{~A})$ & 109.2 \\
\hline $\mathrm{C}(1)-\mathrm{C}(2)-\mathrm{H}(2 \mathrm{~A})$ & 109.2 \\
\hline $\mathrm{C}(3)-\mathrm{C}(2)-\mathrm{H}(2 \mathrm{AB})$ & 109.2 \\
\hline $\mathrm{C}(1)-\mathrm{C}(2)-\mathrm{H}(2 \mathrm{AB})$ & 109.2 \\
\hline $\mathrm{H}(2 \mathrm{~A})-\mathrm{C}(2)-\mathrm{H}(2 \mathrm{AB})$ & 107.9 \\
\hline $\mathrm{O}(1)-\mathrm{C}(3)-\mathrm{C}(4)$ & $118.57(10)$ \\
\hline $\mathrm{O}(1)-\mathrm{C}(3)-\mathrm{C}(2)$ & $120.87(10)$ \\
\hline $\mathrm{C}(4)-\mathrm{C}(3)-\mathrm{C}(2)$ & $120.56(10)$ \\
\hline $\mathrm{C}(5)-\mathrm{C}(4)-\mathrm{C}(9)$ & $116.95(10)$ \\
\hline$C(5)-C(4)-C(3)$ & $125.74(10)$ \\
\hline $\mathrm{C}(9)-\mathrm{C}(4)-\mathrm{C}(3)$ & $117.26(10)$ \\
\hline $\mathrm{F}(1)-\mathrm{C}(5)-\mathrm{C}(6)$ & $117.52(10)$ \\
\hline $\mathrm{F}(1)-\mathrm{C}(5)-\mathrm{C}(4)$ & $120.77(10)$ \\
\hline$C(6)-C(5)-C(4)$ & $121.70(10)$ \\
\hline$C(7)-C(6)-C(5)$ & $120.29(10)$ \\
\hline
\end{tabular}




\begin{tabular}{|c|c|}
\hline $\mathrm{C}(7)-\mathrm{C}(6)-\mathrm{Cl}(1)$ & $120.67(9)$ \\
\hline $\mathrm{C}(5)-\mathrm{C}(6)-\mathrm{Cl}(1)$ & $119.03(9)$ \\
\hline$C(6)-C(7)-C(8)$ & $119.08(11)$ \\
\hline $\mathrm{C}(6)-\mathrm{C}(7)-\mathrm{H}(7)$ & 120.5 \\
\hline $\mathrm{C}(8)-\mathrm{C}(7)-\mathrm{H}(7)$ & 120.5 \\
\hline $\mathrm{C}(9)-\mathrm{C}(8)-\mathrm{C}(7)$ & $120.27(11)$ \\
\hline $\mathrm{C}(9)-\mathrm{C}(8)-\mathrm{H}(8)$ & 119.9 \\
\hline $\mathrm{C}(7)-\mathrm{C}(8)-\mathrm{H}(8)$ & 119.9 \\
\hline$C(8)-C(9)-C(4)$ & $121.71(11)$ \\
\hline $\mathrm{C}(8)-\mathrm{C}(9)-\mathrm{H}(9)$ & 119.1 \\
\hline $\mathrm{C}(4)-\mathrm{C}(9)-\mathrm{H}(9)$ & 119.1 \\
\hline $\mathrm{C}(11)-\mathrm{C}(10)-\mathrm{C}(1)$ & $113.00(9)$ \\
\hline $\mathrm{C}(11)-\mathrm{C}(10)-\mathrm{H}(10 \mathrm{~A})$ & 109.0 \\
\hline $\mathrm{C}(1)-\mathrm{C}(10)-\mathrm{H}(10 \mathrm{~A})$ & 109.0 \\
\hline $\mathrm{C}(11)-\mathrm{C}(10)-\mathrm{H}(10 \mathrm{~B})$ & 109.0 \\
\hline $\mathrm{C}(1)-\mathrm{C}(10)-\mathrm{H}(10 \mathrm{~B})$ & 109.0 \\
\hline $\mathrm{H}(10 \mathrm{~A})-\mathrm{C}(10)-\mathrm{H}(10 \mathrm{~B})$ & 107.8 \\
\hline $\mathrm{O}(2)-\mathrm{C}(11)-\mathrm{C}(12)$ & $118.76(10)$ \\
\hline $\mathrm{O}(2)-\mathrm{C}(11)-\mathrm{C}(10)$ & $121.19(10)$ \\
\hline$C(12)-C(11)-C(10)$ & $120.05(10)$ \\
\hline $\mathrm{C}(13)-\mathrm{C}(12)-\mathrm{C}(17)$ & $117.12(10)$ \\
\hline $\mathrm{C}(13)-\mathrm{C}(12)-\mathrm{C}(11)$ & $125.49(10)$ \\
\hline $\mathrm{C}(17)-\mathrm{C}(12)-\mathrm{C}(11)$ & $117.38(10)$ \\
\hline $\mathrm{F}(2)-\mathrm{C}(13)-\mathrm{C}(14)$ & $117.57(10)$ \\
\hline $\mathrm{F}(2)-\mathrm{C}(13)-\mathrm{C}(12)$ & $120.70(10)$ \\
\hline $\mathrm{C}(14)-\mathrm{C}(13)-\mathrm{C}(12)$ & $121.73(11)$ \\
\hline$C(15)-C(14)-C(13)$ & 119.97(11) \\
\hline $\mathrm{C}(15)-\mathrm{C}(14)-\mathrm{Cl}(2)$ & $119.88(9)$ \\
\hline $\mathrm{C}(13)-\mathrm{C}(14)-\mathrm{Cl}(2)$ & $120.15(9)$ \\
\hline$C(14)-C(15)-C(16)$ & $119.52(11)$ \\
\hline $\mathrm{C}(14)-\mathrm{C}(15)-\mathrm{H}(15)$ & 120.2 \\
\hline $\mathrm{C}(16)-\mathrm{C}(15)-\mathrm{H}(15)$ & 120.2 \\
\hline$C(15)-C(16)-C(17)$ & 119.99(11) \\
\hline $\mathrm{C}(15)-\mathrm{C}(16)-\mathrm{H}(16)$ & 120.0 \\
\hline $\mathrm{C}(17)-\mathrm{C}(16)-\mathrm{H}(16)$ & 120.0 \\
\hline C(16)-C(17)-C(12) & $121.66(11)$ \\
\hline $\mathrm{C}(16)-\mathrm{C}(17)-\mathrm{H}(17)$ & 119.2 \\
\hline $\mathrm{C}(12)-\mathrm{C}(17)-\mathrm{H}(17)$ & 119.2 \\
\hline
\end{tabular}




$\begin{array}{ll}\mathrm{C}(23)-\mathrm{C}(18)-\mathrm{C}(19) & 117.17(10) \\ \mathrm{C}(23)-\mathrm{C}(18)-\mathrm{C}(1) & 121.40(10) \\ \mathrm{C}(19)-\mathrm{C}(18)-\mathrm{C}(1) & 121.43(10) \\ \mathrm{C}(20)-\mathrm{C}(19)-\mathrm{C}(18) & 121.76(11) \\ \mathrm{C}(20)-\mathrm{C}(19)-\mathrm{H}(19) & 119.1 \\ \mathrm{C}(18)-\mathrm{C}(19)-\mathrm{H}(19) & 119.1 \\ \mathrm{C}(19)-\mathrm{C}(20)-\mathrm{C}(21) & 119.97(10) \\ \mathrm{C}(19)-\mathrm{C}(20)-\mathrm{H}(20) & 120.0 \\ \mathrm{C}(21)-\mathrm{C}(20)-\mathrm{H}(20) & 120.0 \\ \mathrm{O}(3)-\mathrm{C}(21)-\mathrm{C}(20) & 115.75(10) \\ \mathrm{O}(3)-\mathrm{C}(21)-\mathrm{C}(22) & 124.57(11) \\ \mathrm{C}(20)-\mathrm{C}(21)-\mathrm{C}(22) & 119.68(11) \\ \mathrm{C}(23)-\mathrm{C}(22)-\mathrm{C}(21) & 119.04(11) \\ \mathrm{C}(23)-\mathrm{C}(22)-\mathrm{H}(22) & 120.5 \\ \mathrm{C}(21)-\mathrm{C}(22)-\mathrm{H}(22) & 120.5 \\ \mathrm{C}(22)-\mathrm{C}(23)-\mathrm{C}(18) & 122.37(10) \\ \mathrm{C}(22)-\mathrm{C}(23)-\mathrm{H}(23) & 118.8 \\ \mathrm{C}(18)-\mathrm{C}(23)-\mathrm{H}(23) & 118.8 \\ \mathrm{O}(3)-\mathrm{C}(24)-\mathrm{H}(24 \mathrm{~A}) & 109.5 \\ \mathrm{O}(3)-\mathrm{C}(24)-\mathrm{H}(24 \mathrm{~B}) & 109.5 \\ \mathrm{H}(24 \mathrm{~A})-\mathrm{C}(24)-\mathrm{H}(24 \mathrm{~B}) & 109.5 \\ \mathrm{O}(3)-\mathrm{C}(24)-\mathrm{H}(24 \mathrm{C}) & 109.5 \\ \mathrm{H}(24 \mathrm{~A})-\mathrm{C}(24)-\mathrm{H}(24 \mathrm{C}) & 109.5 \\ \mathrm{H}(24 \mathrm{~B})-\mathrm{C}(24)-\mathrm{H}(24 \mathrm{C}) & 109.5 \\ & \end{array}$

Symmetry transformations used to generate equivalent atoms: 
Table S20. Anisotropic displacement parameters $\left(\AA^{2} \times 10^{3}\right)$ for i17995. The anisotropic displacement factor exponent takes the form: $-2 \pi^{2}\left[h^{2} a^{* 2} U^{11}+\ldots+2 h k^{*} b^{*} U^{12}\right]$

\begin{tabular}{|c|c|c|c|c|c|c|}
\hline & $\mathrm{U}^{11}$ & $\mathrm{U}^{22}$ & $\mathrm{U}^{33}$ & $\mathrm{U}^{23}$ & $\mathrm{U}^{13}$ & $\mathrm{U}^{12}$ \\
\hline $\mathrm{Cl}(1)$ & $15(1)$ & 19(1) & 19(1) & $-1(1)$ & $-1(1)$ & $-6(1)$ \\
\hline $\mathrm{Cl}(2)$ & $25(1)$ & $24(1)$ & $24(1)$ & $8(1)$ & $-7(1)$ & $-15(1)$ \\
\hline $\mathrm{F}(1)$ & $18(1)$ & $14(1)$ & $25(1)$ & $7(1)$ & $0(1)$ & $-6(1)$ \\
\hline $\mathrm{F}(2)$ & $17(1)$ & $15(1)$ & $25(1)$ & $1(1)$ & $1(1)$ & $-5(1)$ \\
\hline $\mathrm{O}(1)$ & $17(1)$ & $17(1)$ & $24(1)$ & $6(1)$ & $1(1)$ & $-5(1)$ \\
\hline $\mathrm{O}(2)$ & $22(1)$ & $16(1)$ & 19(1) & $1(1)$ & $1(1)$ & $-8(1)$ \\
\hline $\mathrm{O}(3)$ & $17(1)$ & $25(1)$ & $23(1)$ & $-2(1)$ & $-5(1)$ & $-9(1)$ \\
\hline $\mathrm{C}(1)$ & $14(1)$ & $10(1)$ & $16(1)$ & $2(1)$ & $-3(1)$ & $-4(1)$ \\
\hline$C(2)$ & $13(1)$ & 11(1) & $17(1)$ & $3(1)$ & $-2(1)$ & $-4(1)$ \\
\hline$C(3)$ & $14(1)$ & $13(1)$ & $17(1)$ & $3(1)$ & $-5(1)$ & $-5(1)$ \\
\hline C(4) & $14(1)$ & $12(1)$ & $16(1)$ & $3(1)$ & $-5(1)$ & $-5(1)$ \\
\hline$C(5)$ & $15(1)$ & $13(1)$ & $17(1)$ & $5(1)$ & $-5(1)$ & $-6(1)$ \\
\hline$C(6)$ & $14(1)$ & $15(1)$ & $15(1)$ & $0(1)$ & $-3(1)$ & $-5(1)$ \\
\hline$C(7)$ & $19(1)$ & $12(1)$ & $23(1)$ & $2(1)$ & $-6(1)$ & $-5(1)$ \\
\hline$C(8)$ & $23(1)$ & $14(1)$ & $24(1)$ & $6(1)$ & $-4(1)$ & $-7(1)$ \\
\hline $\mathrm{C}(9)$ & $17(1)$ & $14(1)$ & $20(1)$ & $5(1)$ & $-3(1)$ & $-6(1)$ \\
\hline$C(10)$ & $14(1)$ & 11(1) & $18(1)$ & 1(1) & $-2(1)$ & $-4(1)$ \\
\hline $\mathrm{C}(11)$ & $15(1)$ & $12(1)$ & $16(1)$ & 1(1) & $-5(1)$ & $-6(1)$ \\
\hline$C(12)$ & $16(1)$ & $12(1)$ & $16(1)$ & $1(1)$ & $-5(1)$ & $-6(1)$ \\
\hline$C(13)$ & $15(1)$ & $12(1)$ & $17(1)$ & $0(1)$ & $-5(1)$ & $-4(1)$ \\
\hline$C(14)$ & $19(1)$ & $18(1)$ & $19(1)$ & $4(1)$ & $-7(1)$ & $-11(1)$ \\
\hline$C(15)$ & $24(1)$ & $13(1)$ & $26(1)$ & $5(1)$ & $-10(1)$ & $-8(1)$ \\
\hline$C(16)$ & $21(1)$ & $13(1)$ & $28(1)$ & $0(1)$ & $-5(1)$ & $-4(1)$ \\
\hline $\mathrm{C}(17)$ & $17(1)$ & $14(1)$ & $20(1)$ & $0(1)$ & $-3(1)$ & $-5(1)$ \\
\hline$C(18)$ & $13(1)$ & $9(1)$ & $16(1)$ & $2(1)$ & $-2(1)$ & $-3(1)$ \\
\hline$C(19)$ & $15(1)$ & $12(1)$ & $14(1)$ & $0(1)$ & $-1(1)$ & $-5(1)$ \\
\hline$C(20)$ & $14(1)$ & $14(1)$ & $18(1)$ & $-1(1)$ & $0(1)$ & $-5(1)$ \\
\hline$C(21)$ & $14(1)$ & $11(1)$ & $20(1)$ & 1(1) & $-4(1)$ & $-4(1)$ \\
\hline$C(22)$ & $17(1)$ & $15(1)$ & $15(1)$ & $2(1)$ & $-3(1)$ & $-6(1)$ \\
\hline$C(23)$ & $13(1)$ & $14(1)$ & $16(1)$ & $2(1)$ & $-1(1)$ & $-4(1)$ \\
\hline$C(24)$ & $28(1)$ & $22(1)$ & $24(1)$ & $2(1)$ & $-11(1)$ & $-14(1)$ \\
\hline
\end{tabular}



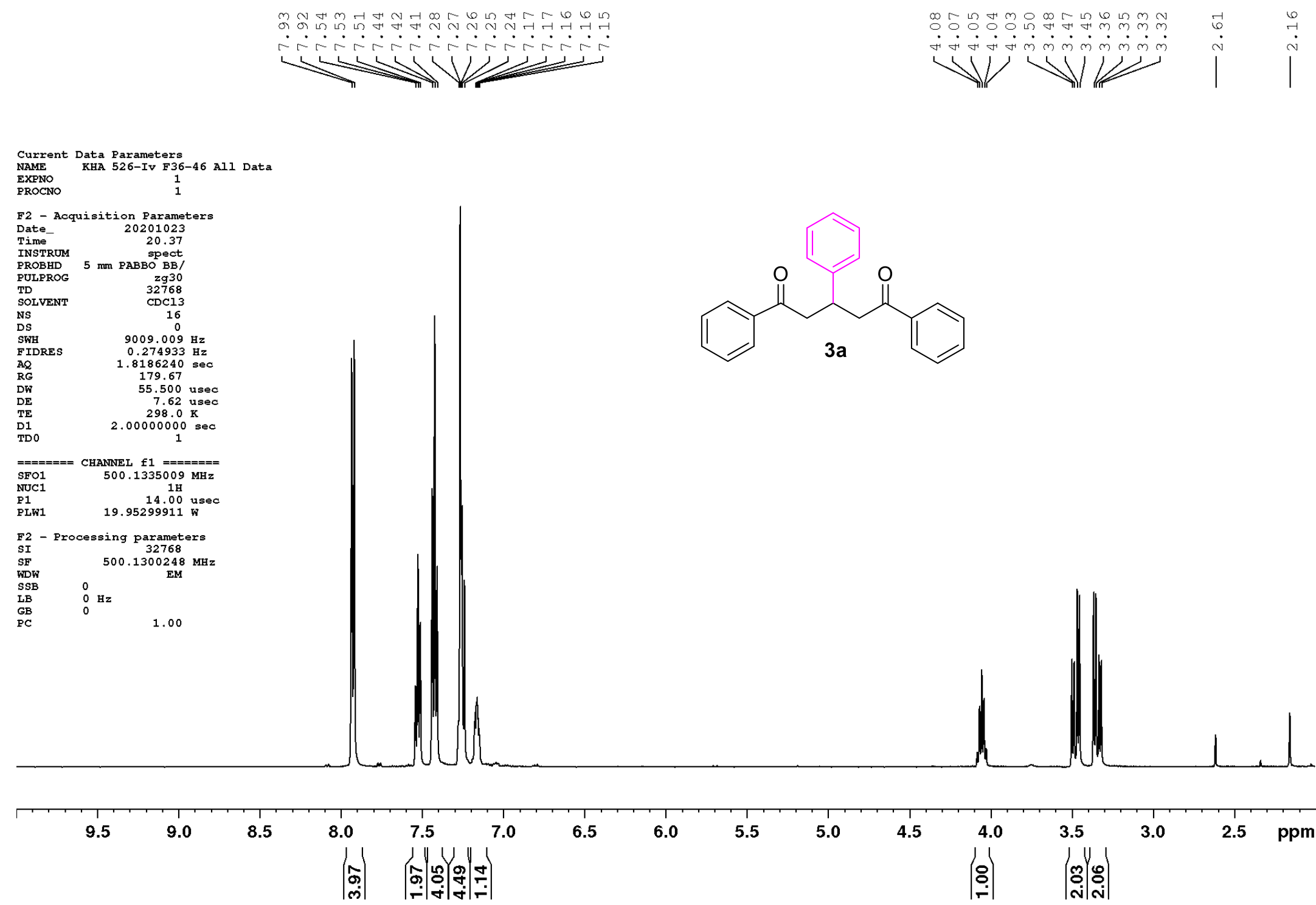

Figure S6. ${ }^{1} \mathrm{H}$ NMR spectrum of compound 3a $\left(500 \mathrm{MHz}, \mathrm{CDCl}_{3}, 25{ }^{\circ} \mathrm{C}\right)$ 


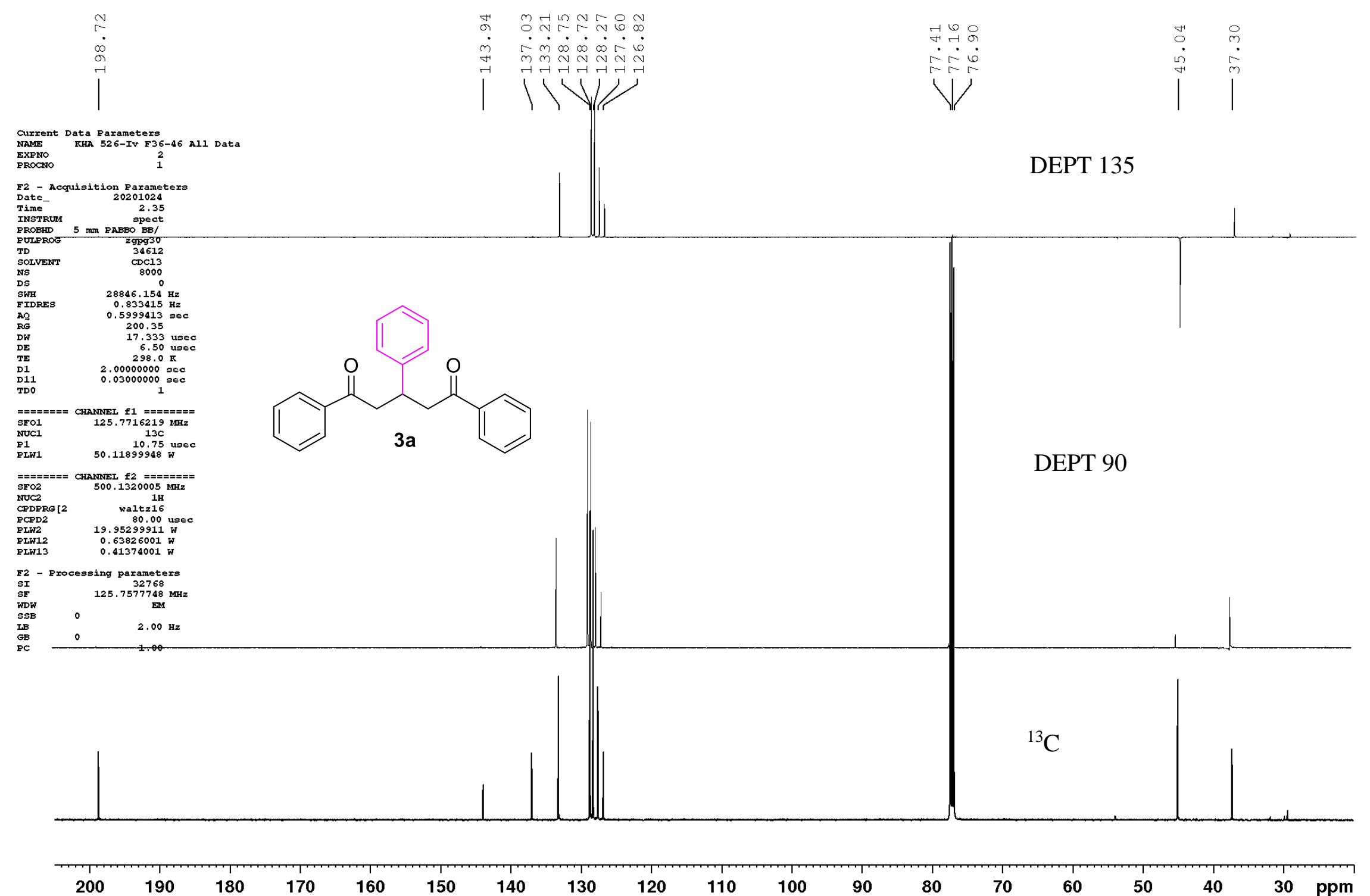

Figure S7. ${ }^{13} \mathrm{C}$ NMR spectrum of compound 3a $\left(125 \mathrm{MHz}, \mathrm{CDCl}_{3}, 25{ }^{\circ} \mathrm{C}\right)$ 


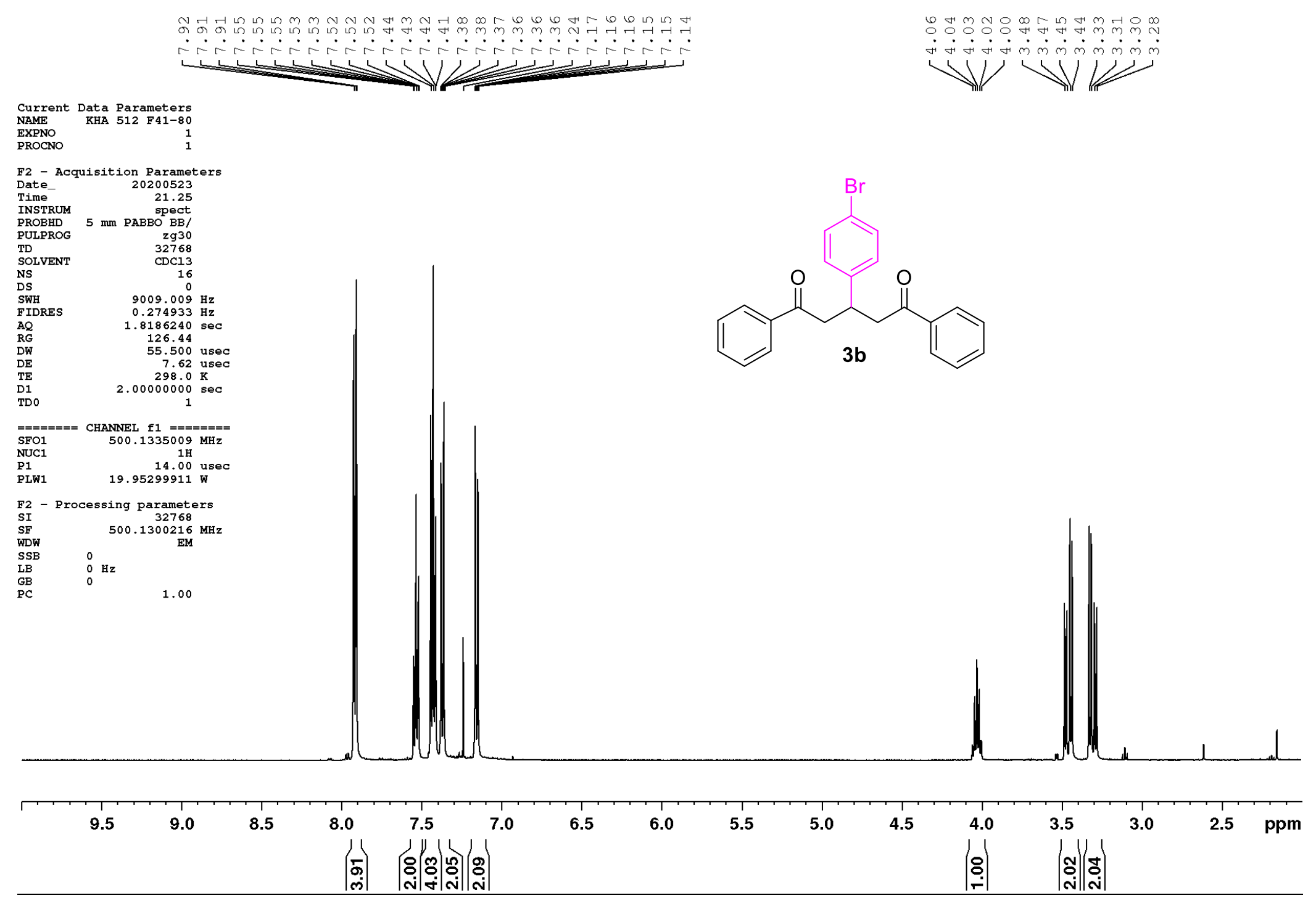

Figure S8. ${ }^{1} \mathrm{H}$ NMR spectrum of compound $3 b\left(500 \mathrm{MHz}, \mathrm{CDCl}_{3}, 25{ }^{\circ} \mathrm{C}\right)$ 

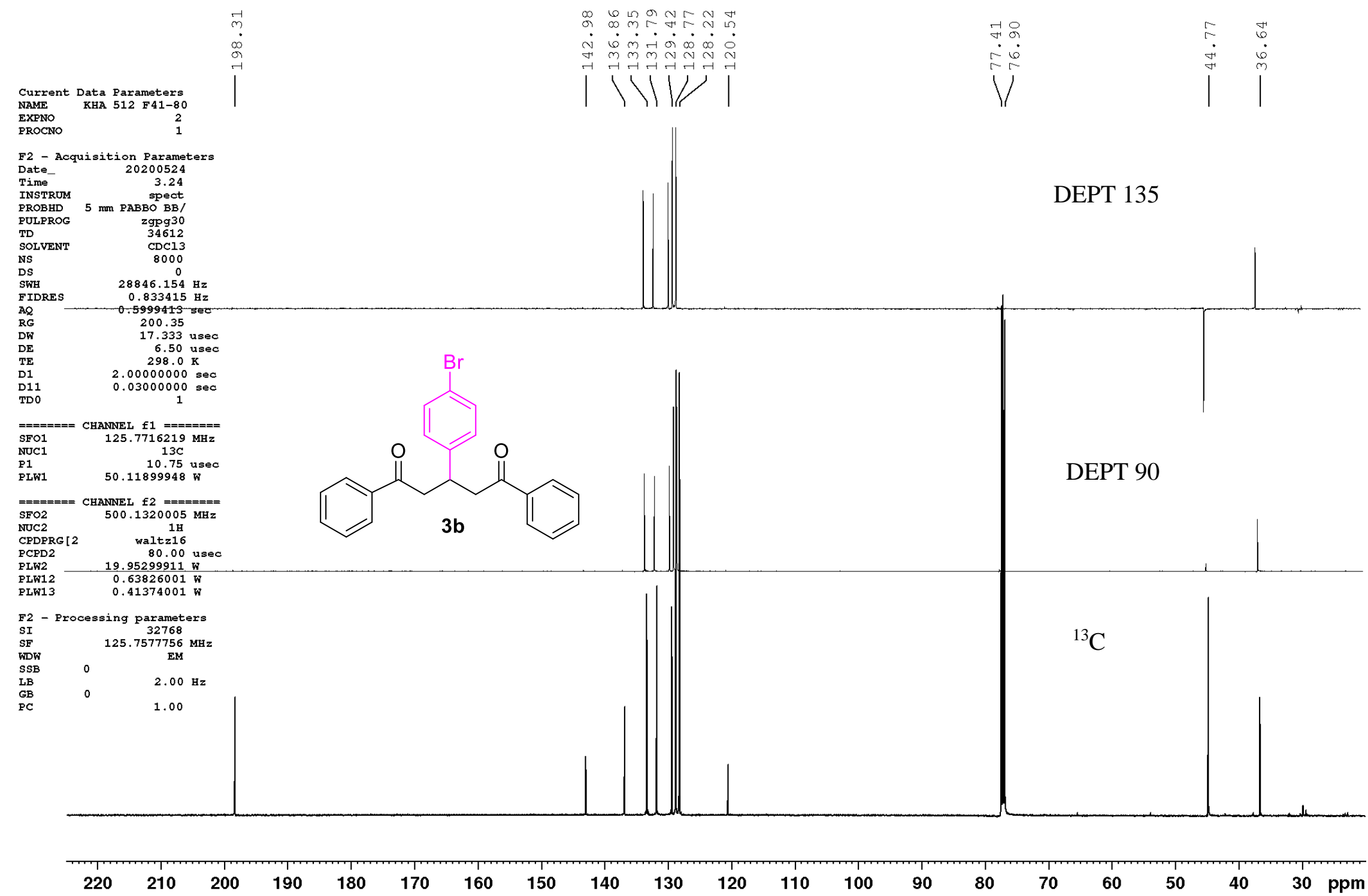

Figure S9. ${ }^{13} \mathrm{C}$ NMR spectrum of compound $3 \mathbf{b}\left(125 \mathrm{MHz}, \mathrm{CDCl}_{3}, 25{ }^{\circ} \mathrm{C}\right)$ 


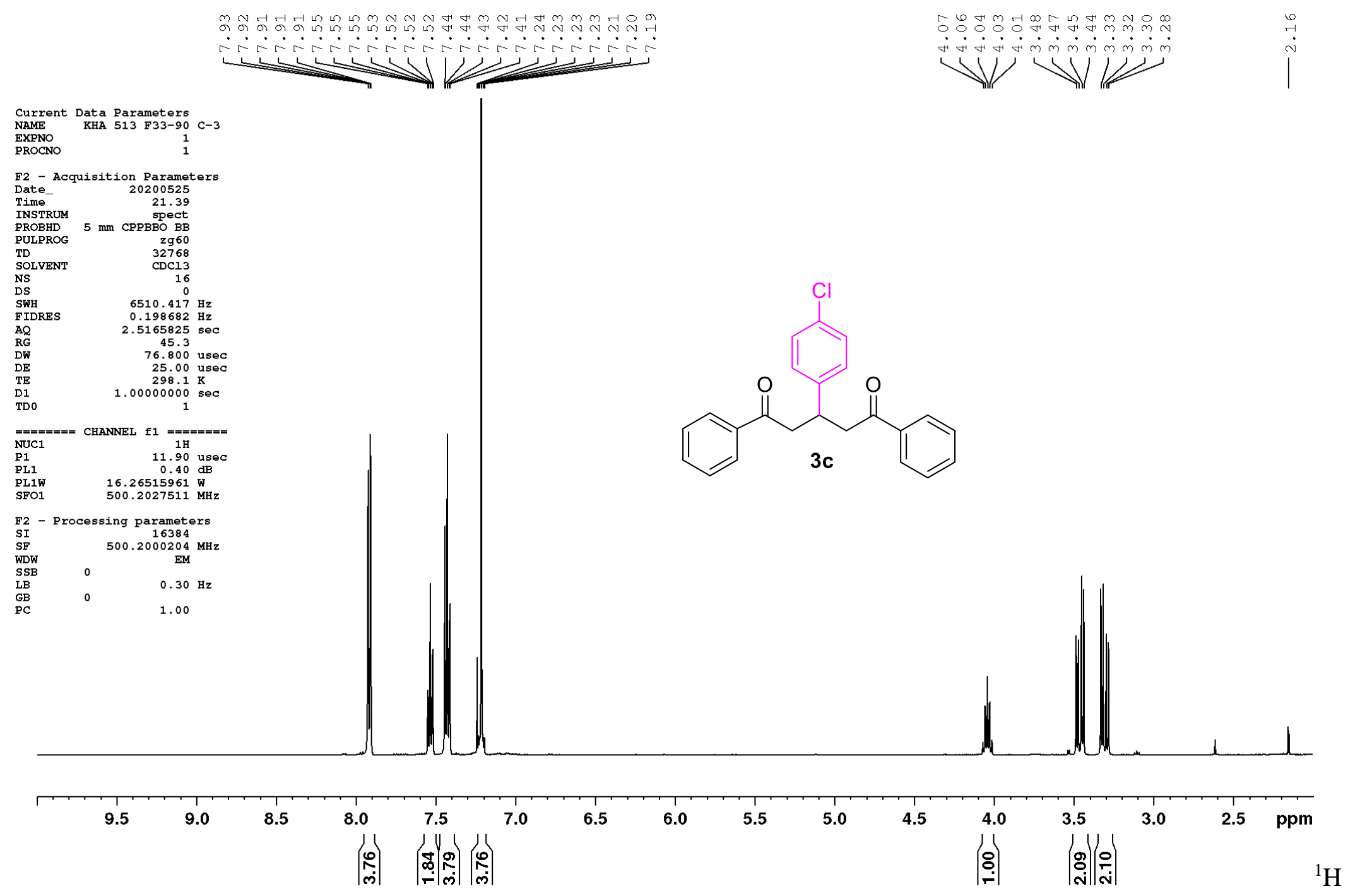

Figure S10. ${ }^{1} \mathrm{H}$ NMR spectrum of compound $3 \mathrm{c}\left(500 \mathrm{MHz}, \mathrm{CDCl}_{3}, 25{ }^{\circ} \mathrm{C}\right)$ 

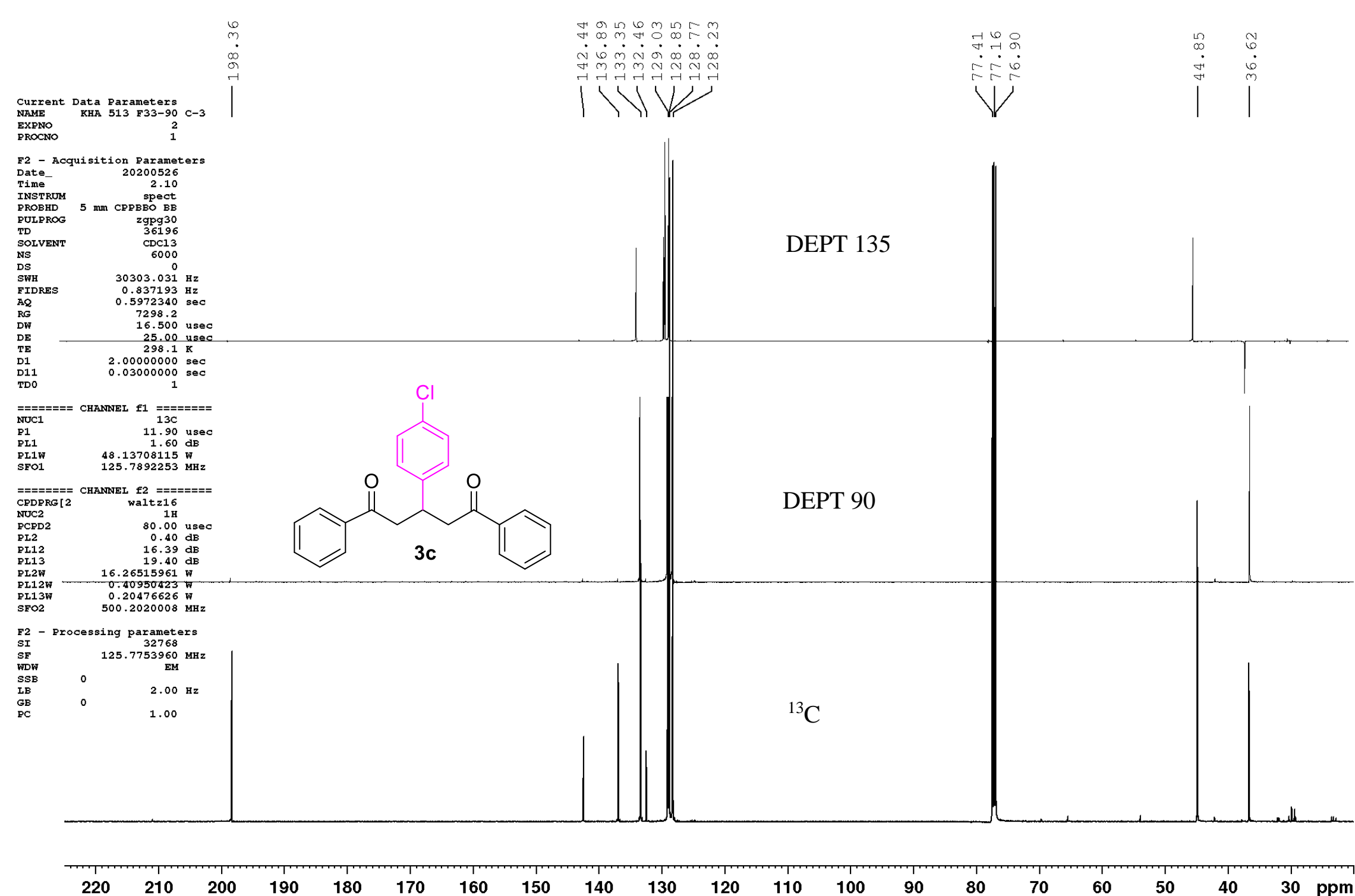

Figure S11. ${ }^{13} \mathrm{C}$ NMR spectrum of compound $3 \mathbf{c}\left(125 \mathrm{MHz}, \mathrm{CDCl}_{3}, 25{ }^{\circ} \mathrm{C}\right)$ 


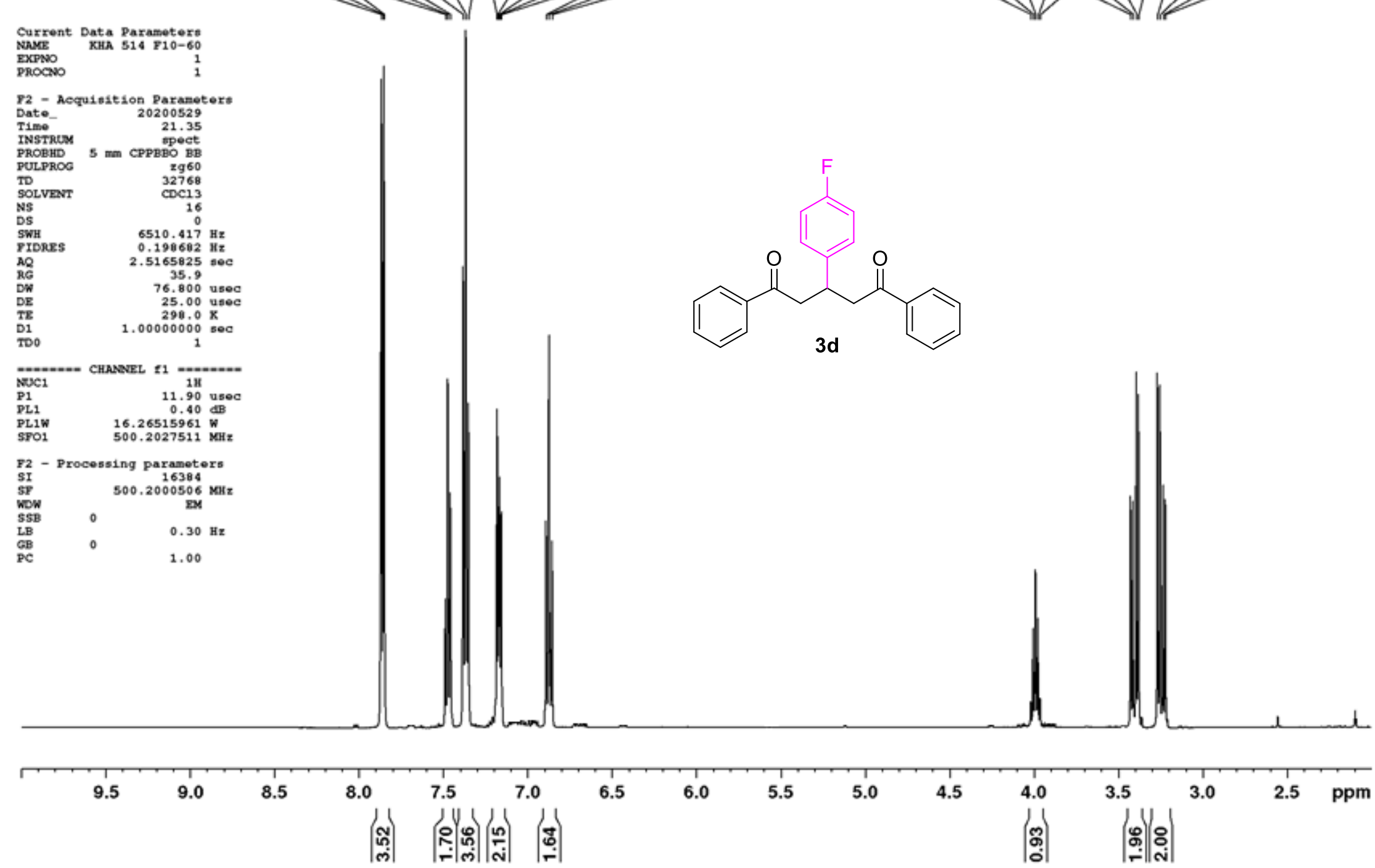

Figure S12. ${ }^{1} \mathrm{H}$ NMR spectrum of compound $\mathbf{3 d}\left(500 \mathrm{MHz}, \mathrm{CDCl}_{3}, 25{ }^{\circ} \mathrm{C}\right)$ 

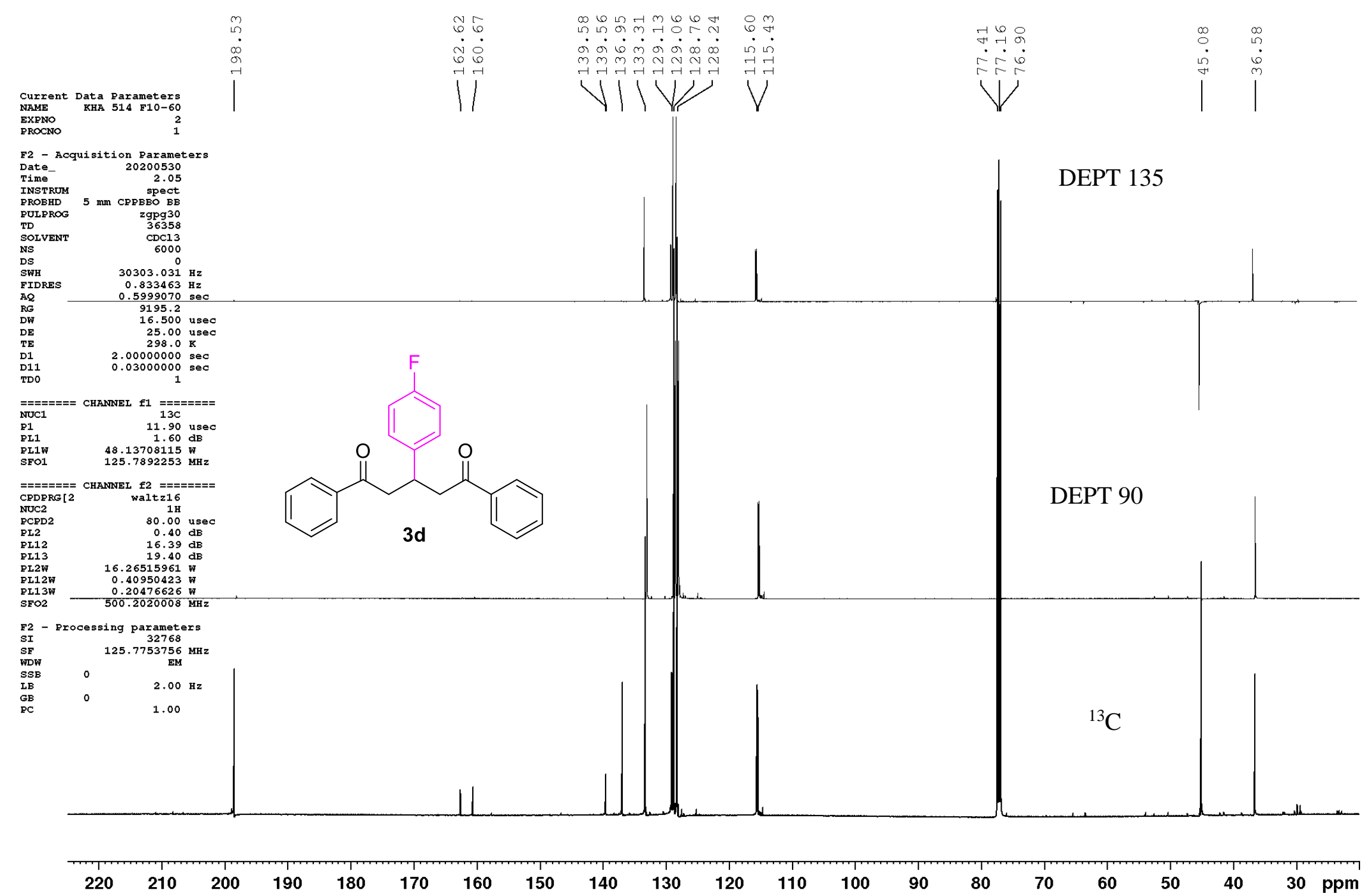

Figure S13. ${ }^{13} \mathrm{C}$ NMR spectrum of compound $\mathbf{3 d}\left(125 \mathrm{MHz}, \mathrm{CDCl}_{3}, 25^{\circ} \mathrm{C}\right)$ 


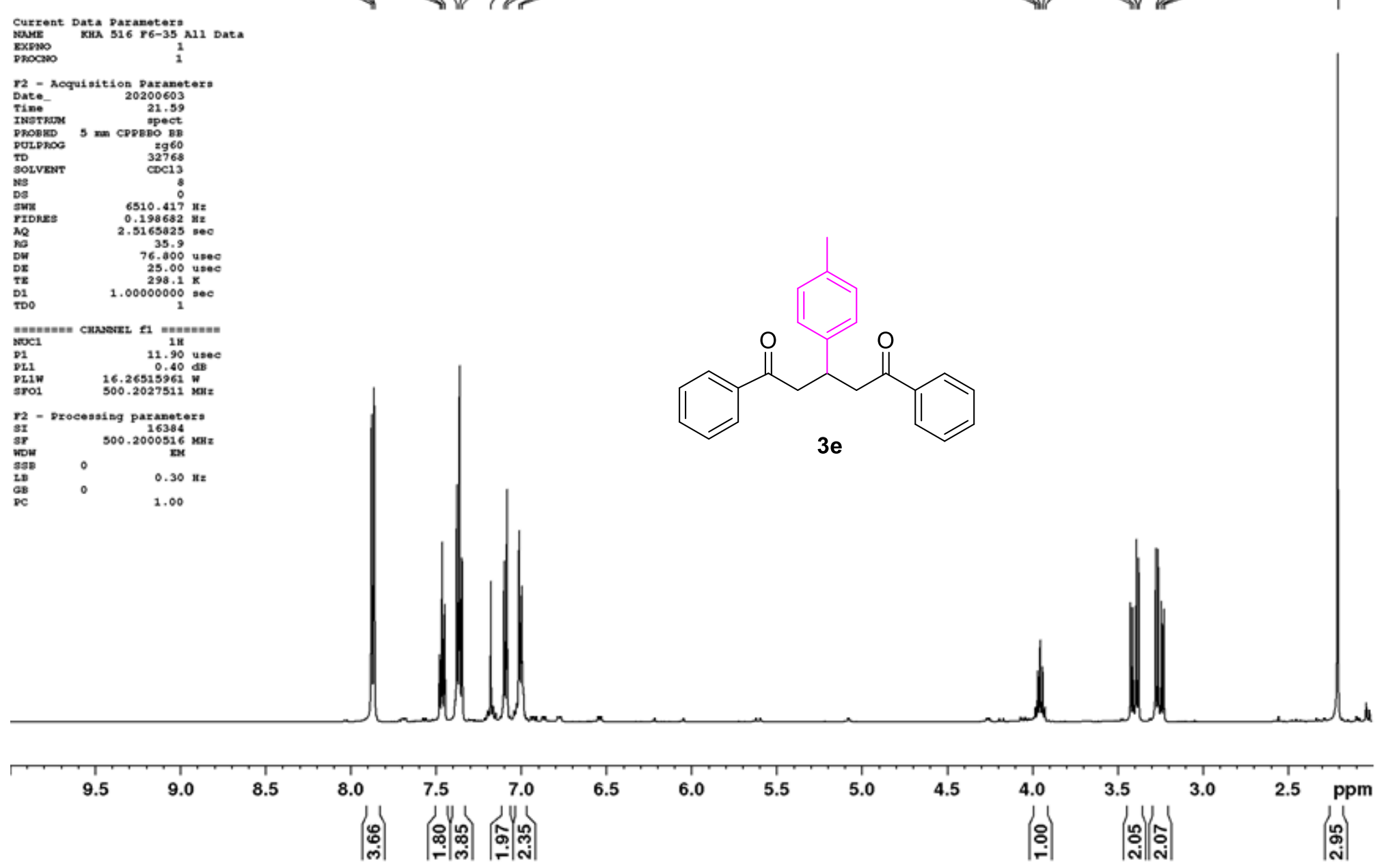

Figure S14. ${ }^{1} \mathrm{H}$ NMR spectrum of compound $3 \mathbf{e}\left(500 \mathrm{MHz}, \mathrm{CDCl}_{3}, 25{ }^{\circ} \mathrm{C}\right)$ 

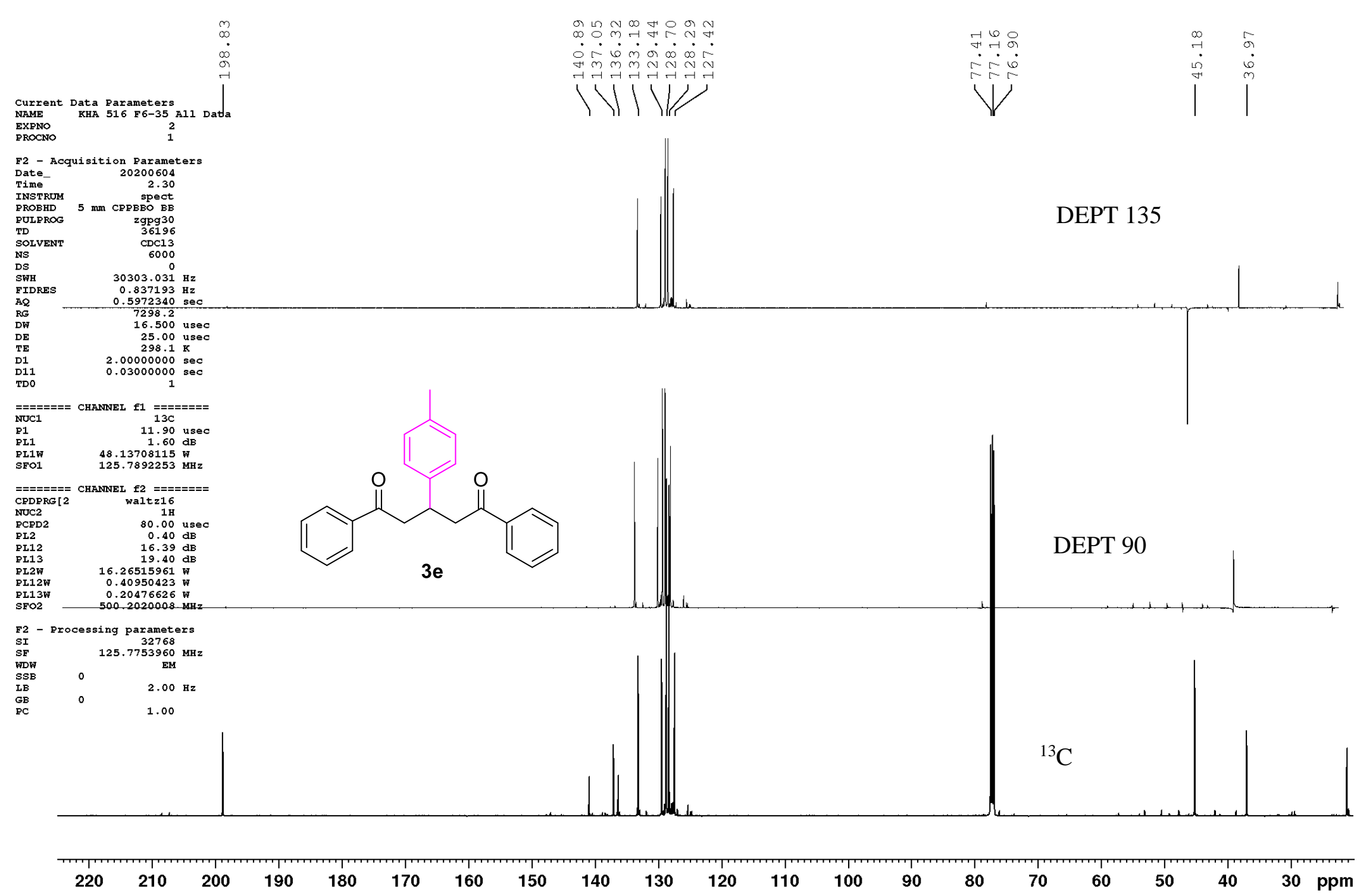

Figure S15. ${ }^{13} \mathrm{C}$ NMR spectrum of compound $3 \mathbf{e}\left(125 \mathrm{MHz}, \mathrm{CDCl}_{3}, 25^{\circ} \mathrm{C}\right)$ 


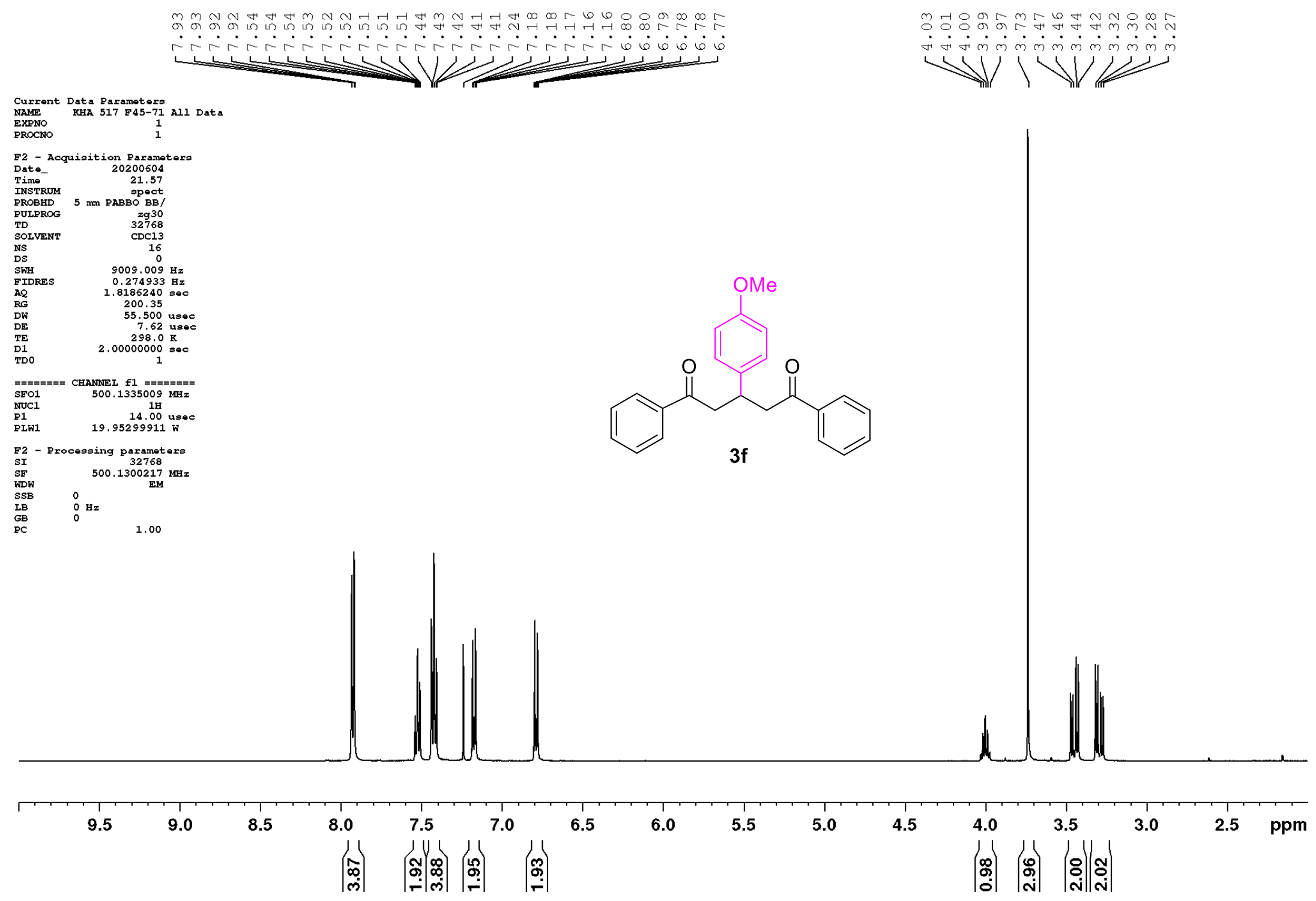

Figure S16. ${ }^{1} \mathrm{H}$ NMR spectrum of compound $3 f\left(500 \mathrm{MHz}, \mathrm{CDCl}_{3}, 25{ }^{\circ} \mathrm{C}\right)$ 

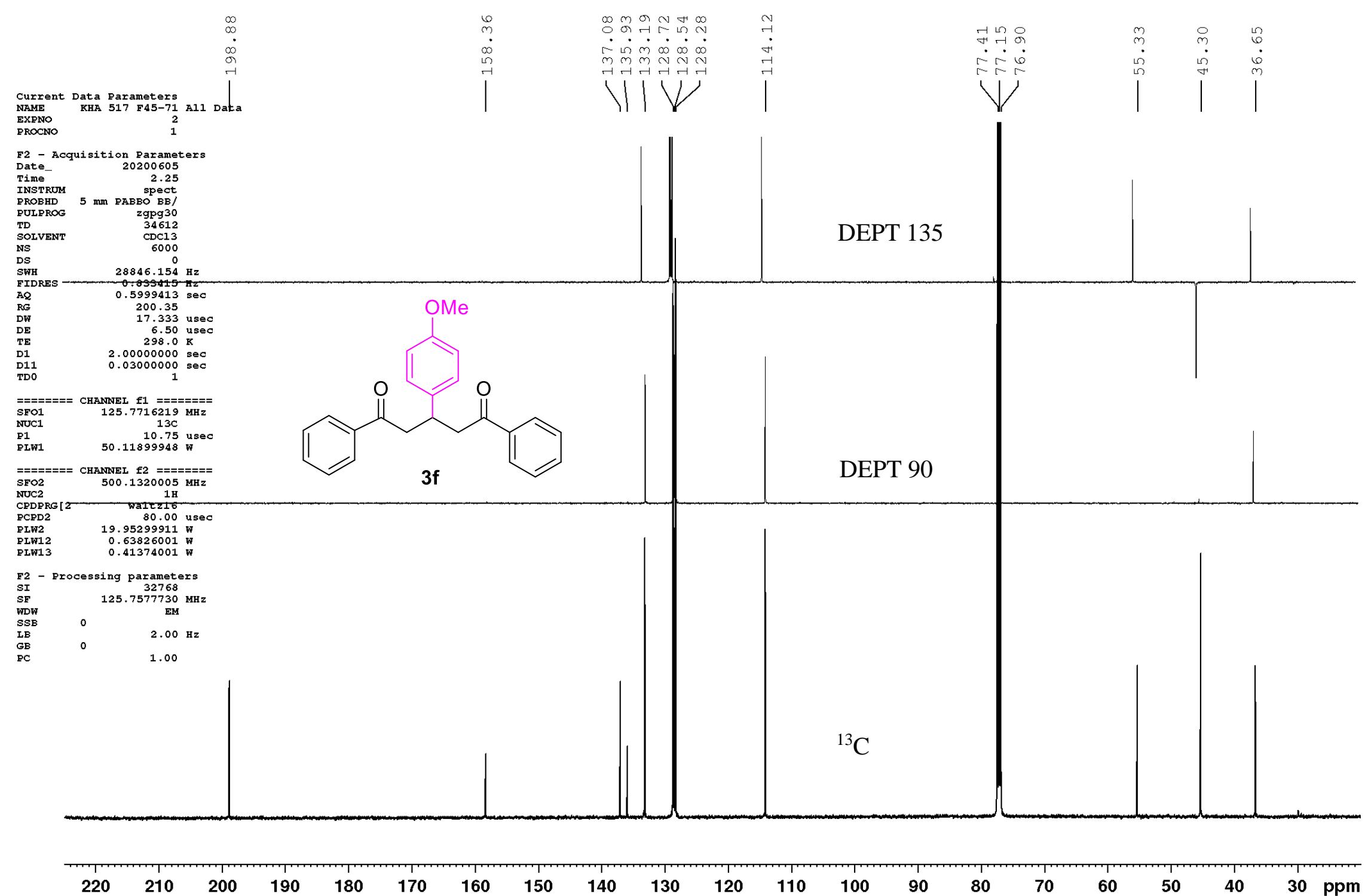

180
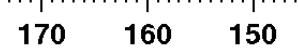

$140 \quad 130$

100

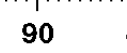

70

60

50

$30 \mathrm{ppm}$

Figure S17. ${ }^{13} \mathrm{C}$ NMR spectrum of compound $3 f\left(125 \mathrm{MHz}, \mathrm{CDCl}_{3}, 25{ }^{\circ} \mathrm{C}\right)$ 


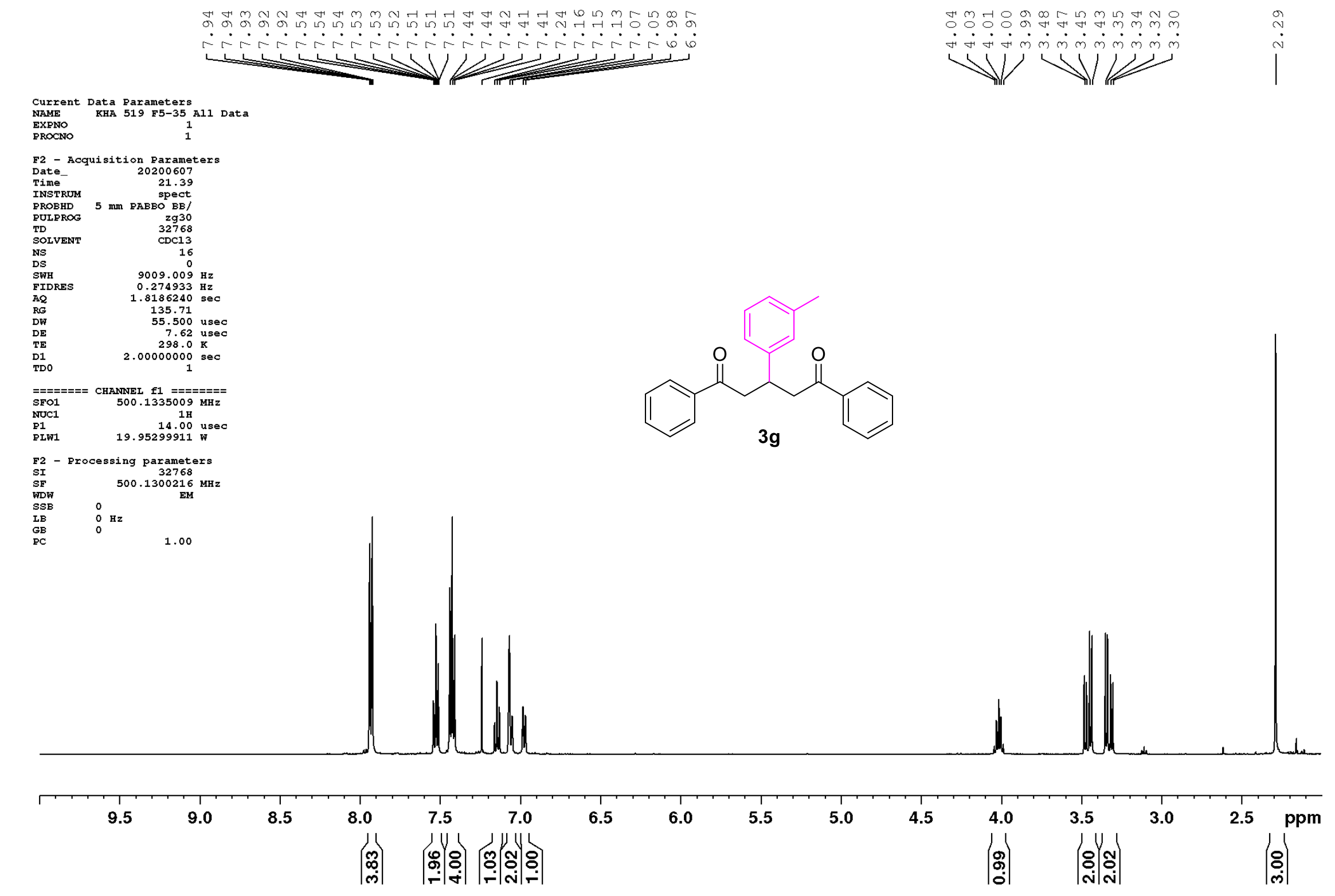

Figure S18. ${ }^{1} \mathrm{H}$ NMR spectrum of compound $3 \mathrm{~g}\left(500 \mathrm{MHz}, \mathrm{CDCl}_{3}, 25{ }^{\circ} \mathrm{C}\right)$ 


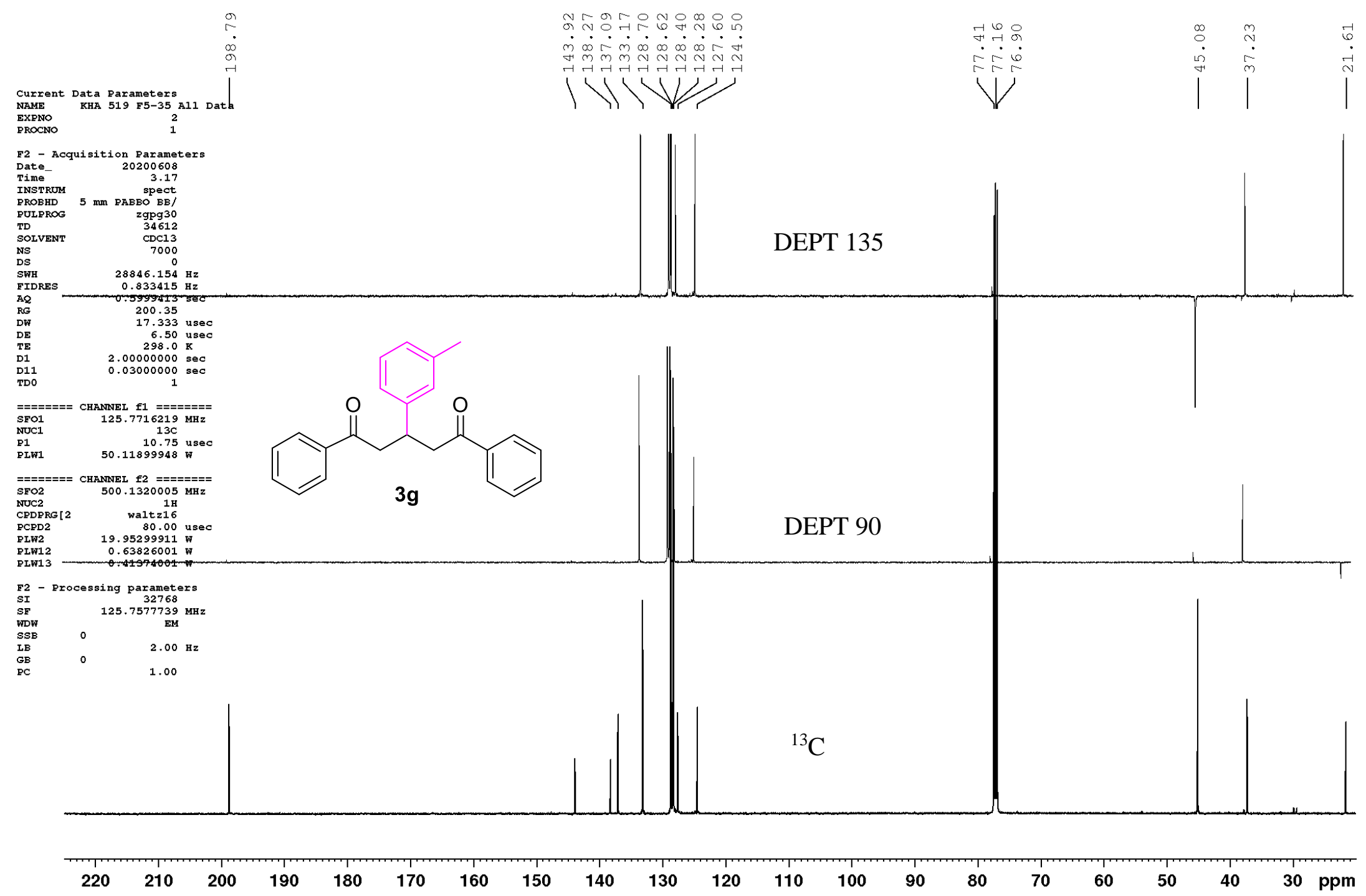

Figure S19. ${ }^{13} \mathrm{C}$ NMR spectrum of compound $3 g\left(125 \mathrm{MHz}, \mathrm{CDCl}_{3}, 25{ }^{\circ} \mathrm{C}\right)$ 


\section{Current Data Parameters
NAME
KHA 520 F4-26 A11 Data}

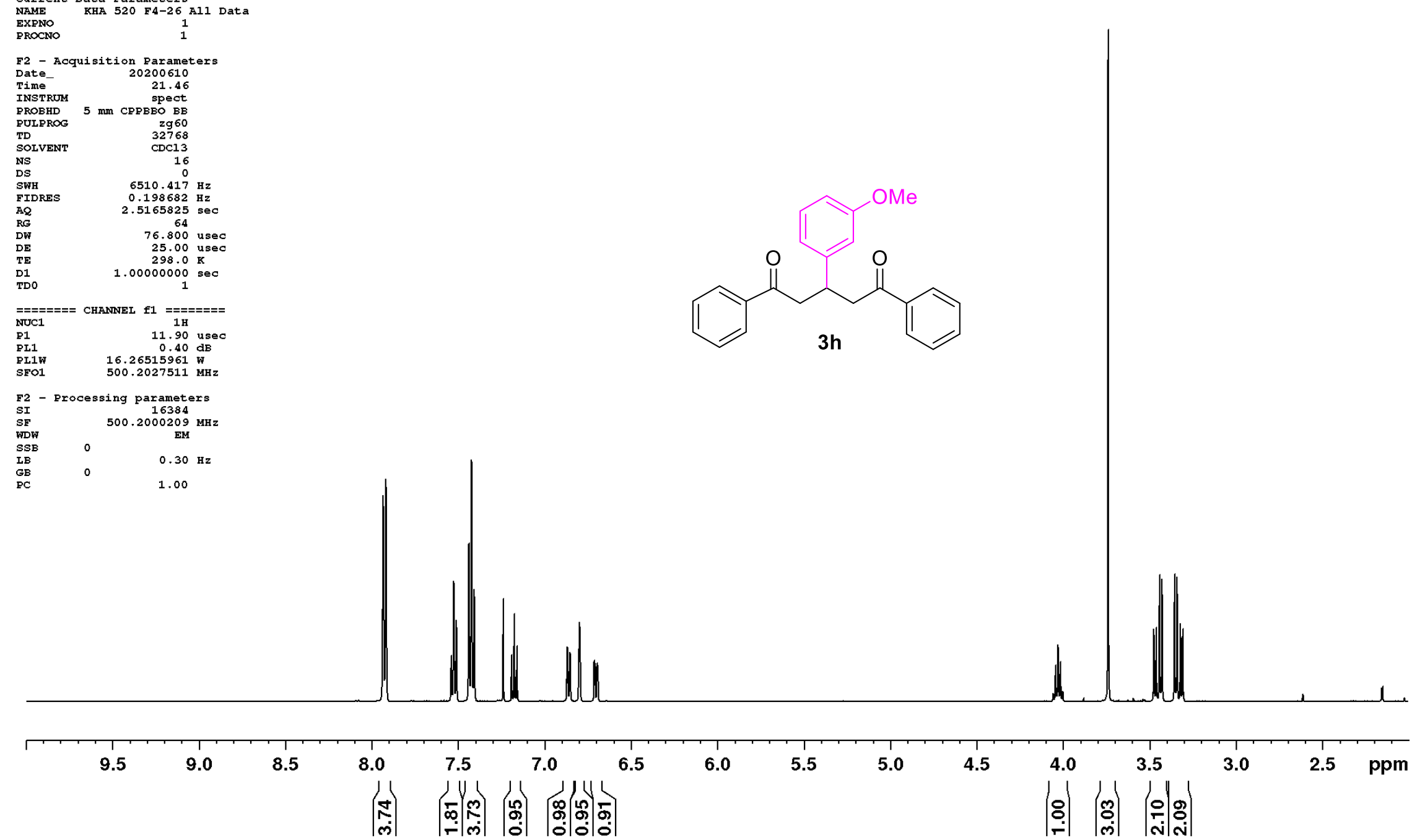

Figure S20. ${ }^{1} \mathrm{H}$ NMR spectrum of compound $\mathbf{3 h}\left(500 \mathrm{MHz}, \mathrm{CDCl}_{3}, 25{ }^{\circ} \mathrm{C}\right)$ 


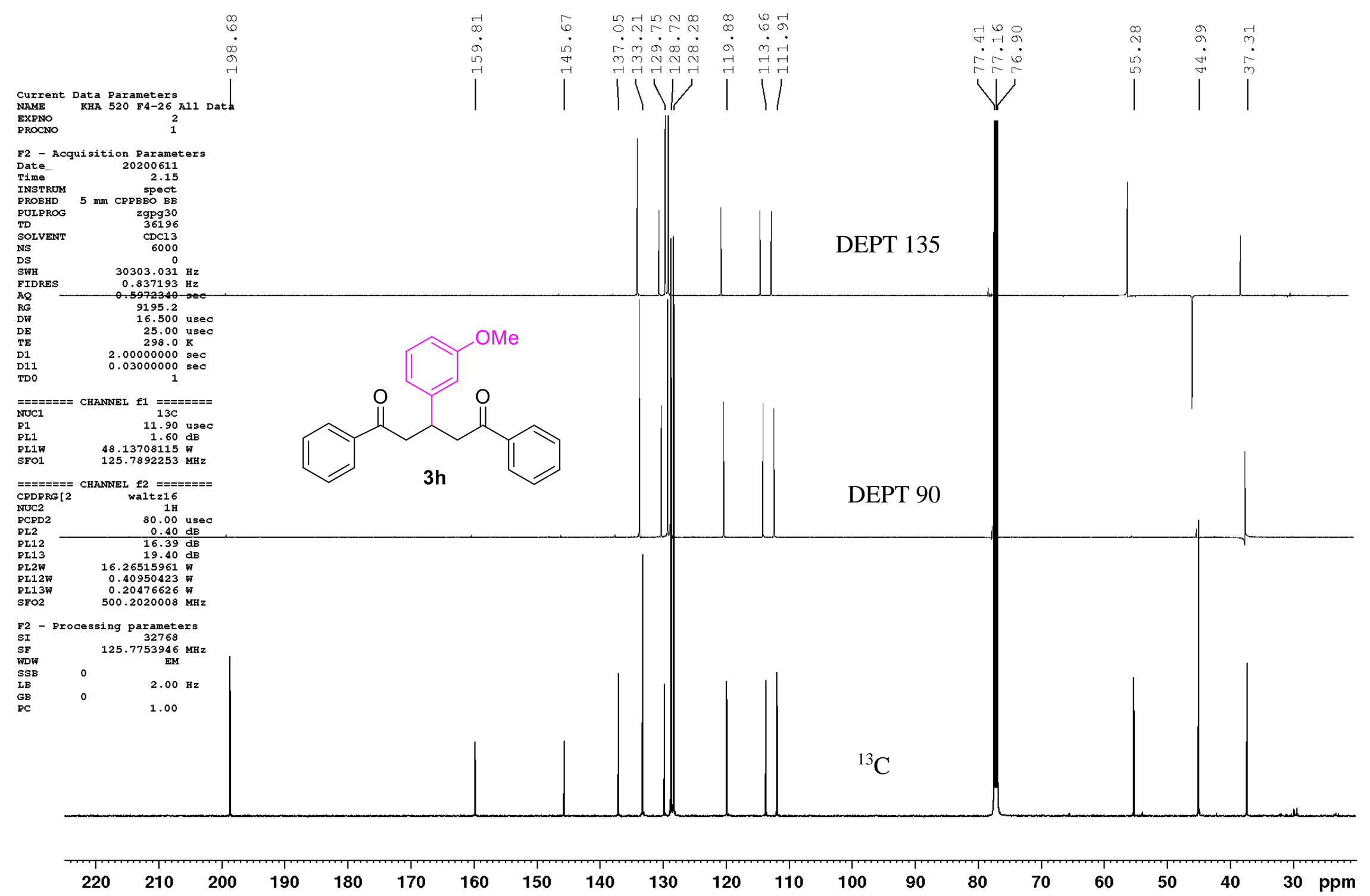

Figure S21. ${ }^{13} \mathrm{C}$ NMR spectrum of compound $\mathbf{3 h}\left(125 \mathrm{MHz}, \mathrm{CDCl}_{3}, 25{ }^{\circ} \mathrm{C}\right)$ 


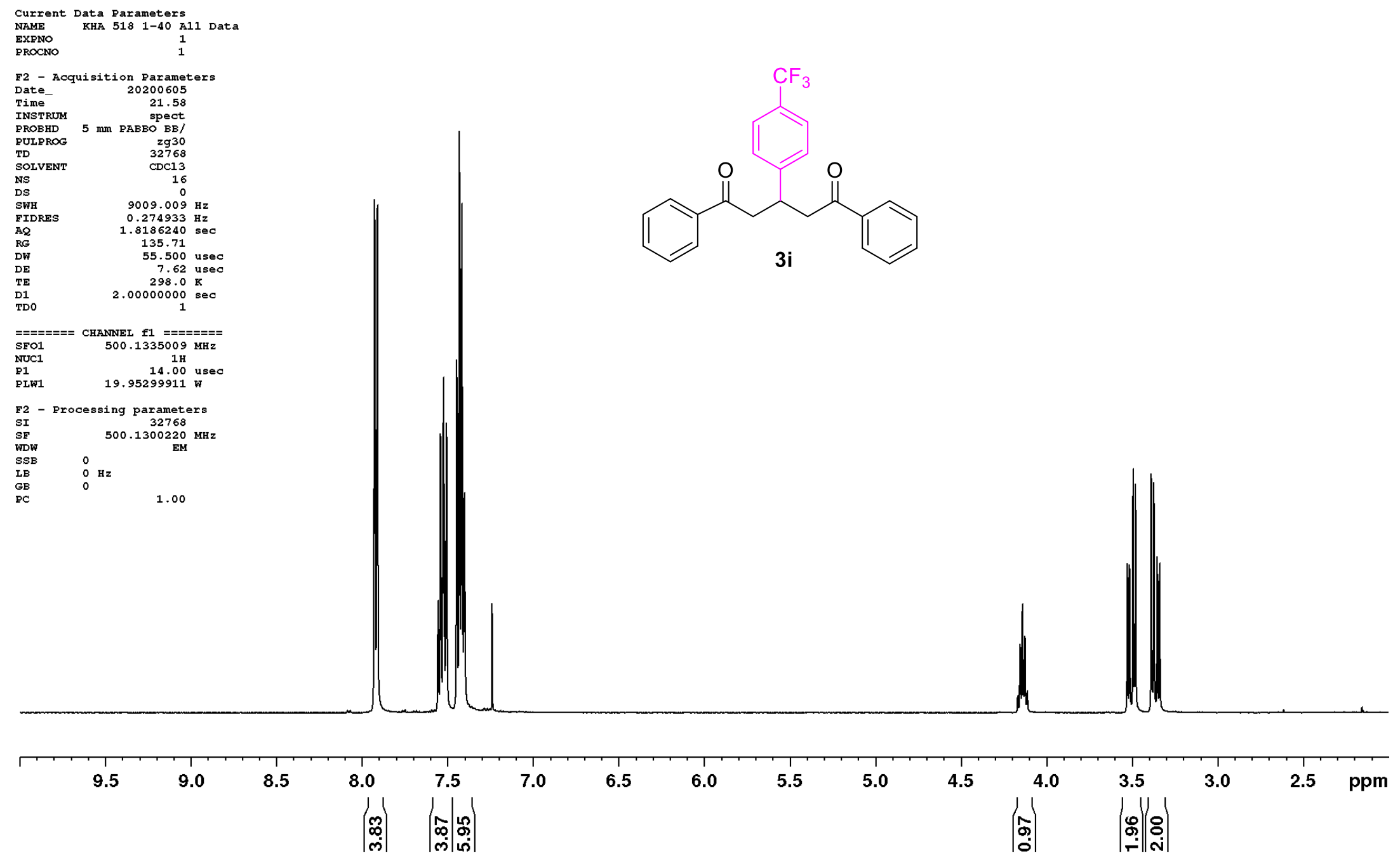

Figure S22. ${ }^{1} \mathrm{H}$ NMR spectrum of compound $3 \mathbf{i}\left(500 \mathrm{MHz}, \mathrm{CDCl}_{3}, 25{ }^{\circ} \mathrm{C}\right.$ ) 

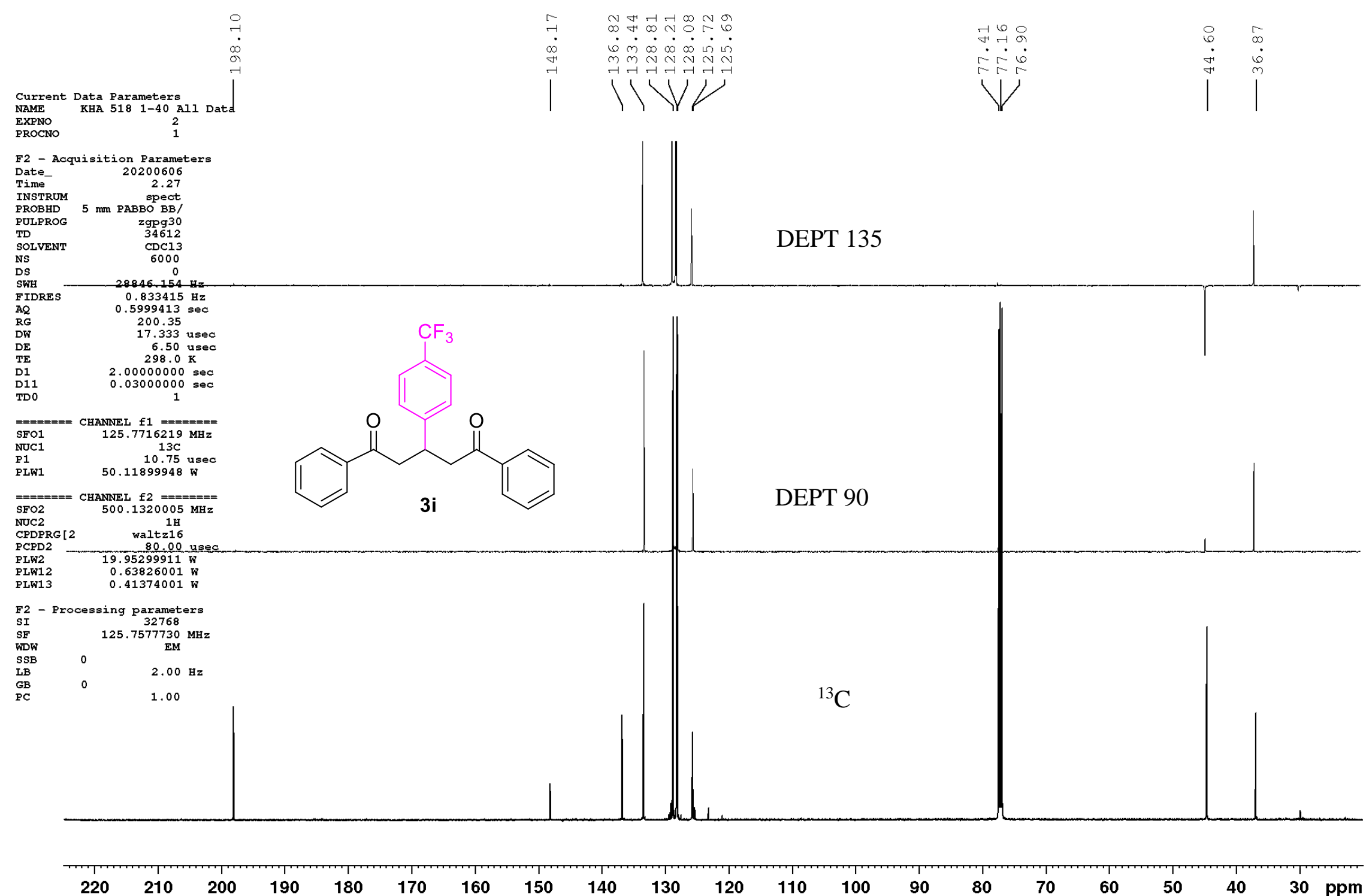

180

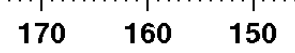

$140 \quad 130$

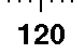

110

100

$90 \quad 80$

70

60

$50 \quad 40$

30 ppm

Figure S23. ${ }^{13} \mathrm{C}$ NMR spectrum of compound $3 \mathbf{i}\left(125 \mathrm{MHz}, \mathrm{CDCl}_{3}, 25{ }^{\circ} \mathrm{C}\right)$ 


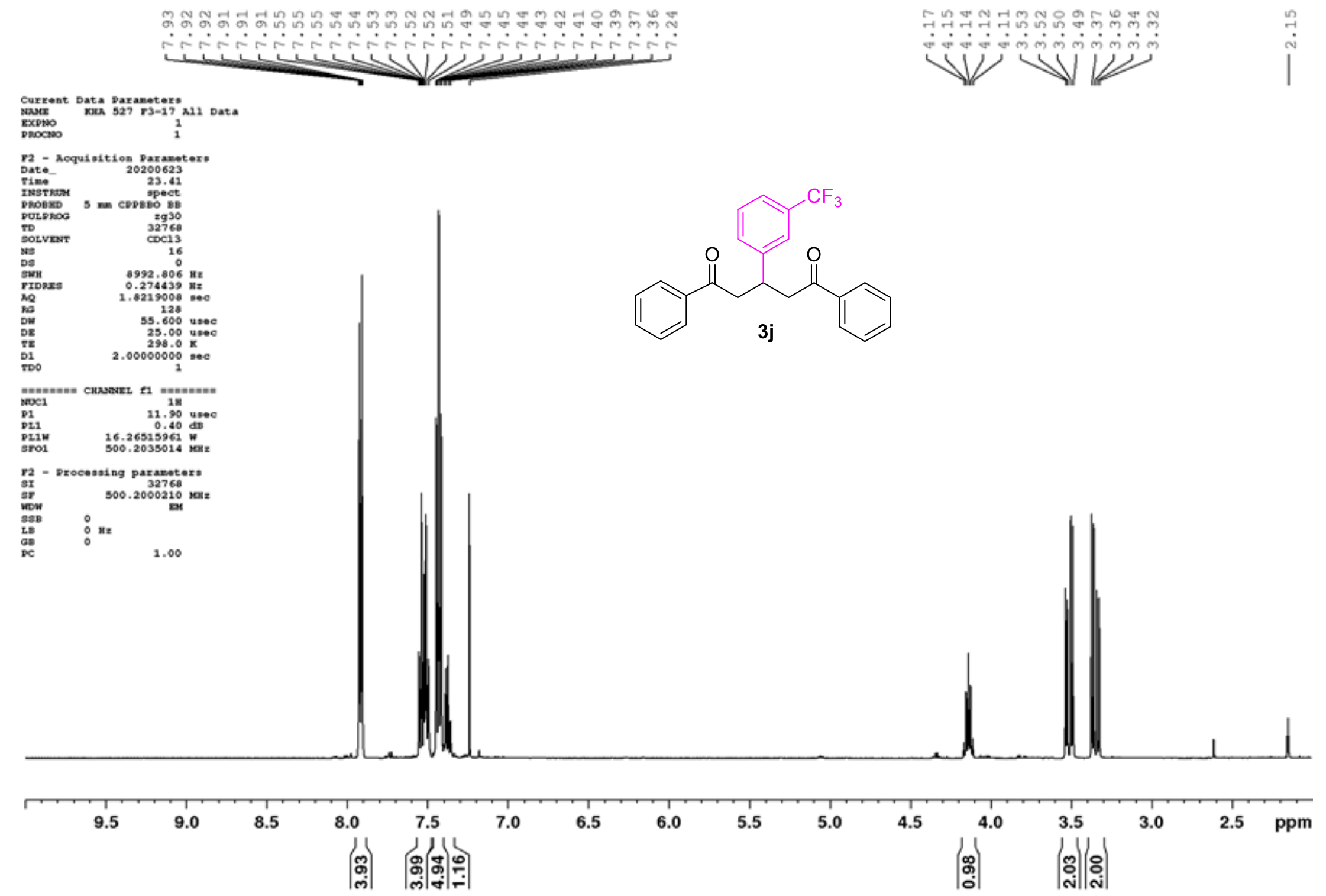

Figure S24. ${ }^{1} \mathrm{H}$ NMR spectrum of compound 3j (500 $\mathrm{MHz}, \mathrm{CDCl}_{3}, 25{ }^{\circ} \mathrm{C}$ ) 


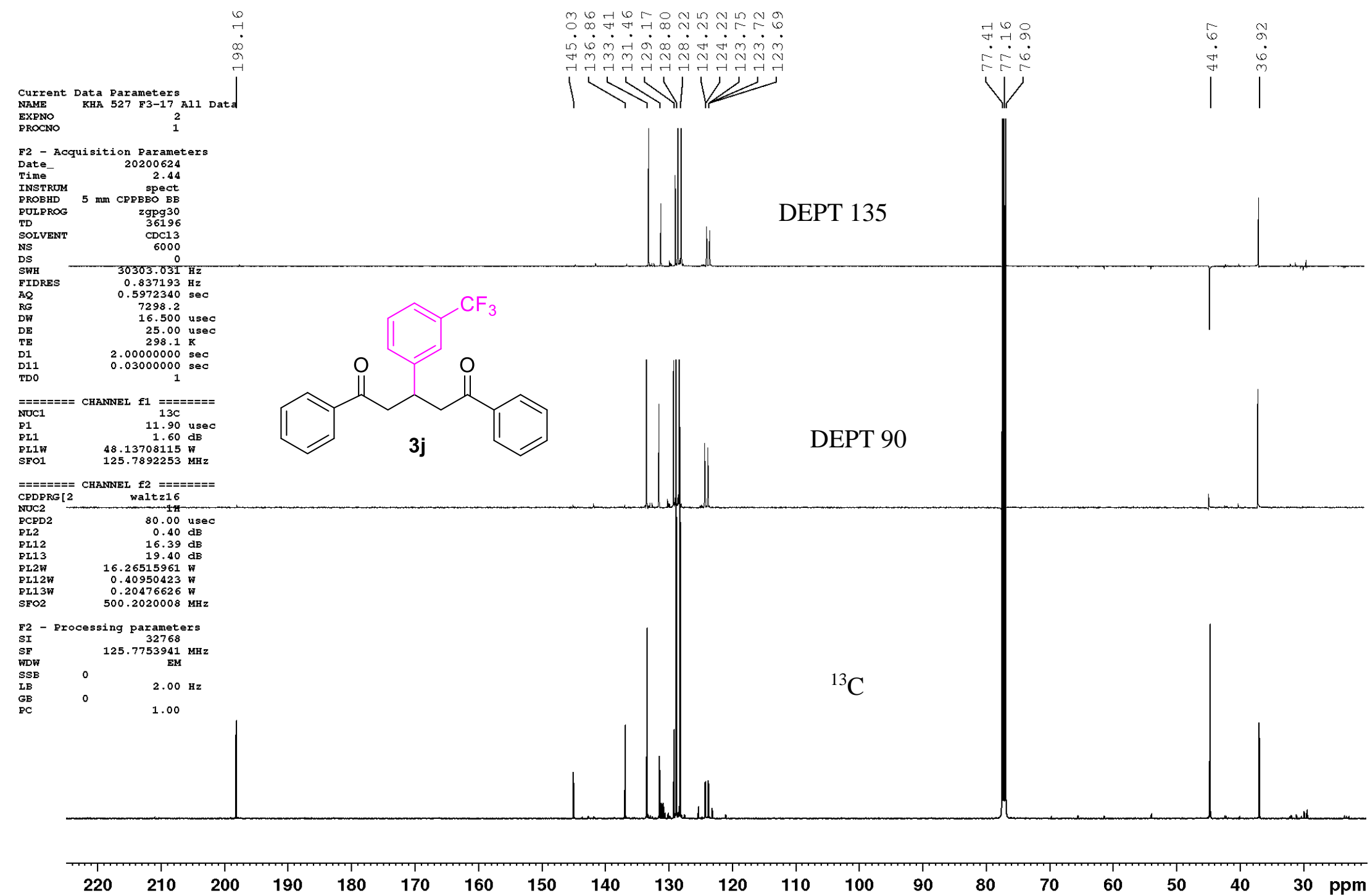

Figure S25. ${ }^{13} \mathrm{C}$ NMR spectrum of compound 3j (125 $\mathrm{MHz}, \mathrm{CDCl}_{3}, 25{ }^{\circ} \mathrm{C}$ ) 


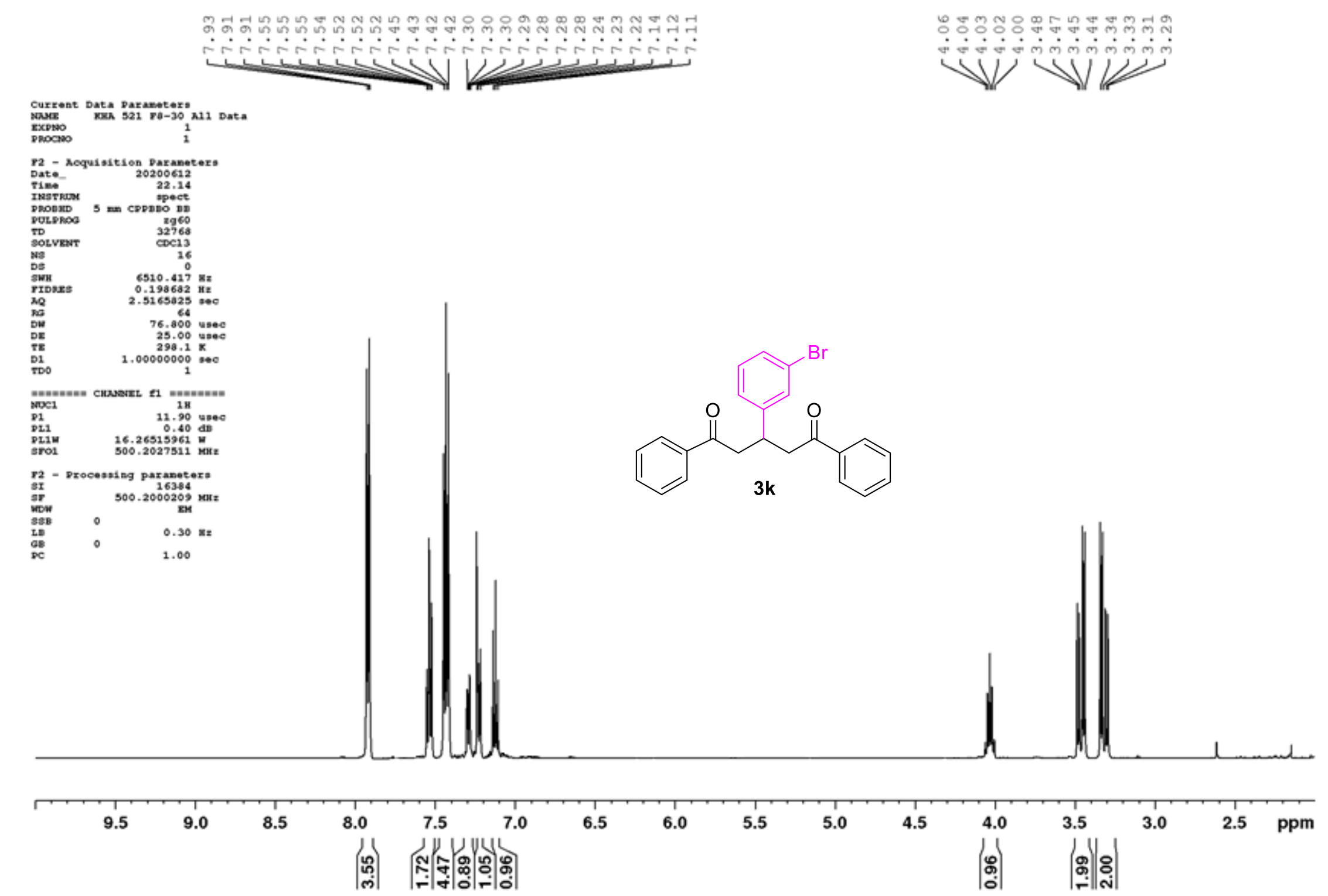

Figure S26. ${ }^{1} \mathrm{H}$ NMR spectrum of compound 3k $\left(500 \mathrm{MHz}, \mathrm{CDCl}_{3}, 25{ }^{\circ} \mathrm{C}\right)$ 


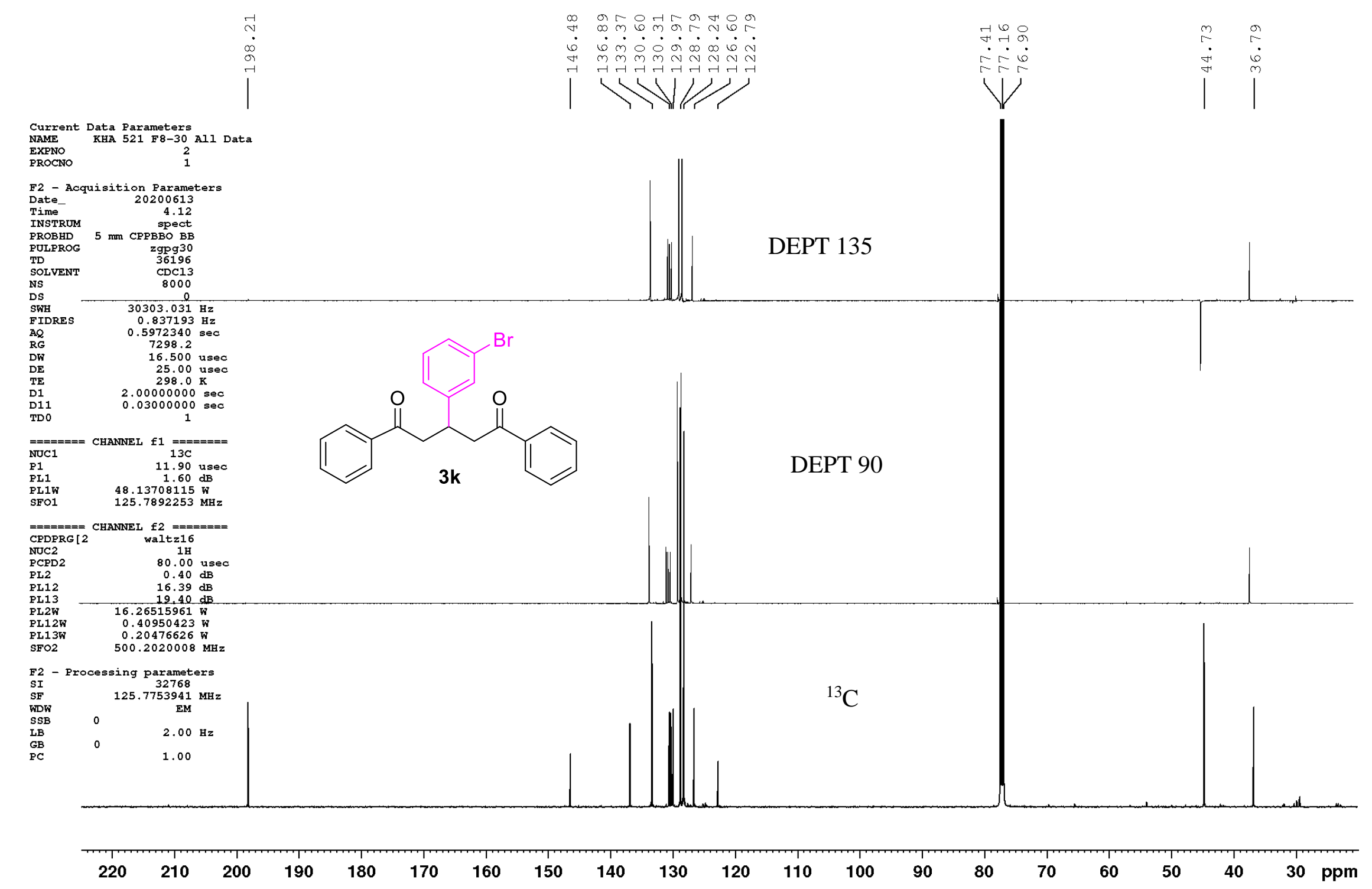

Figure S27. ${ }^{13} \mathrm{C}$ NMR spectrum of compound $\mathbf{3 k}\left(125 \mathrm{MHz}, \mathrm{CDCl}_{3}, 25{ }^{\circ} \mathrm{C}\right)$ 


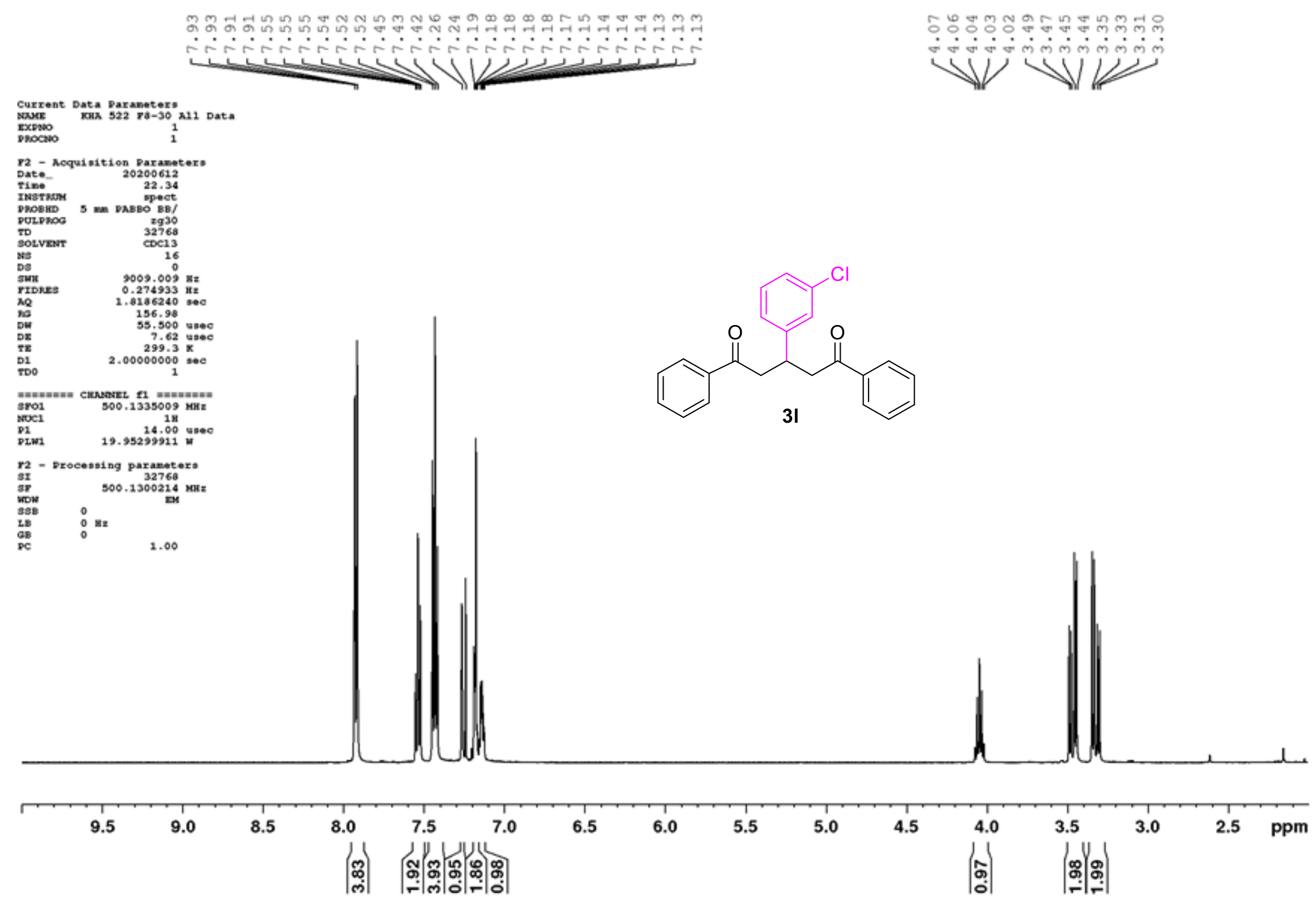

Figure S28. ${ }^{1} \mathrm{H}$ NMR spectrum of compound $31\left(500 \mathrm{MHz}, \mathrm{CDCl}_{3}, 25{ }^{\circ} \mathrm{C}\right)$ 


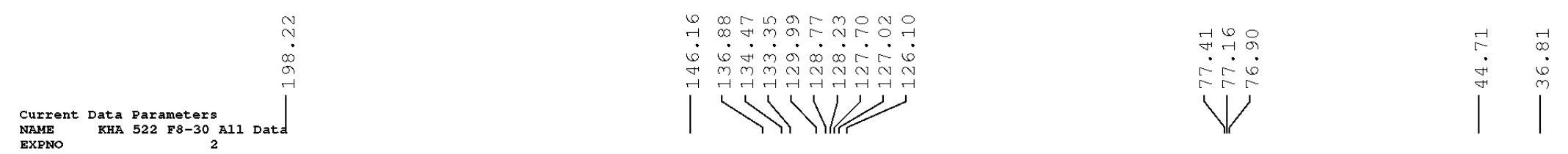

EXPNO
PROCNO

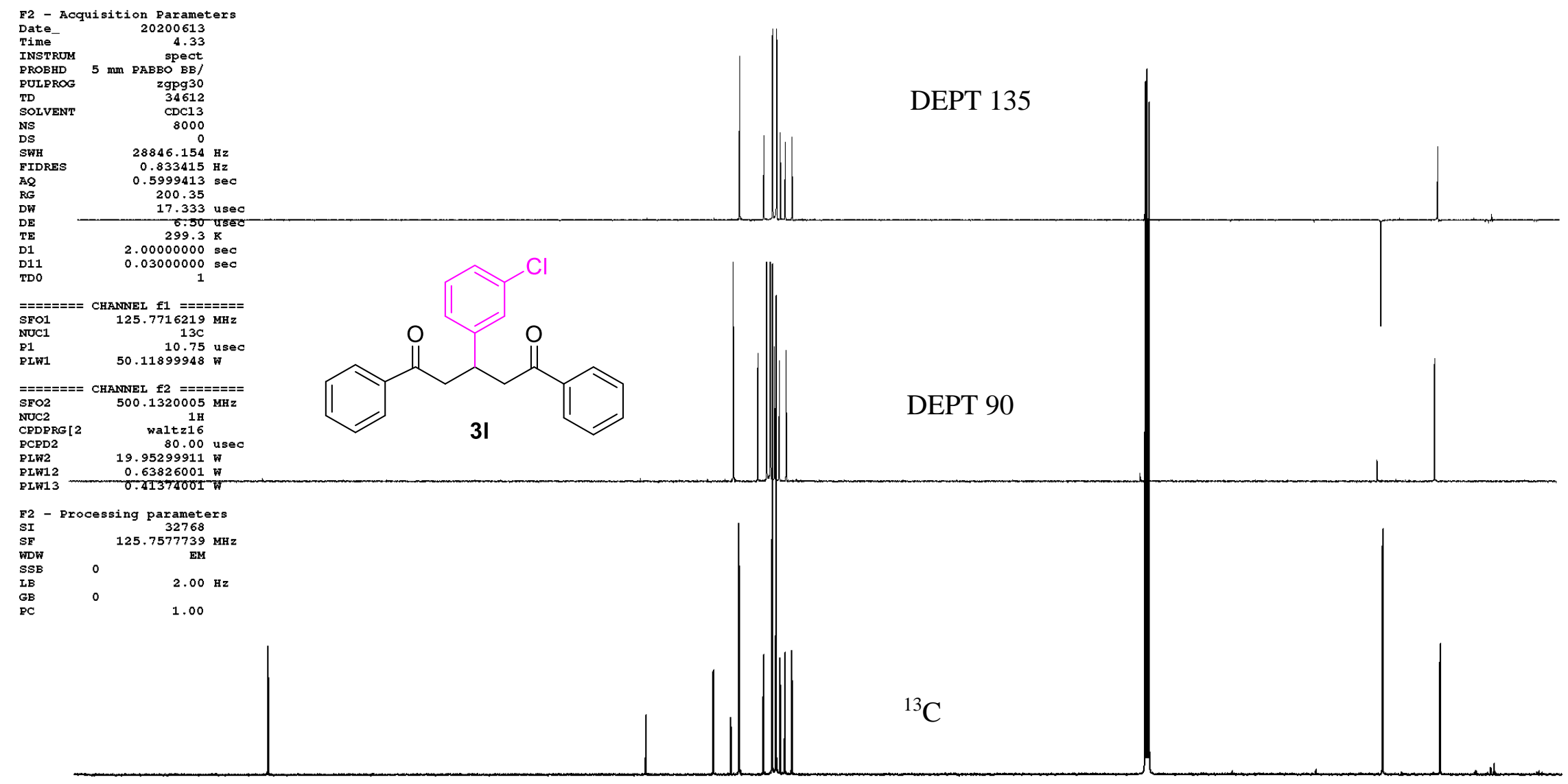

Figure S29. ${ }^{13} \mathrm{C}$ NMR spectrum of compound $31\left(125 \mathrm{MHz}, \mathrm{CDCl}_{3}, 25{ }^{\circ} \mathrm{C}\right)$ 


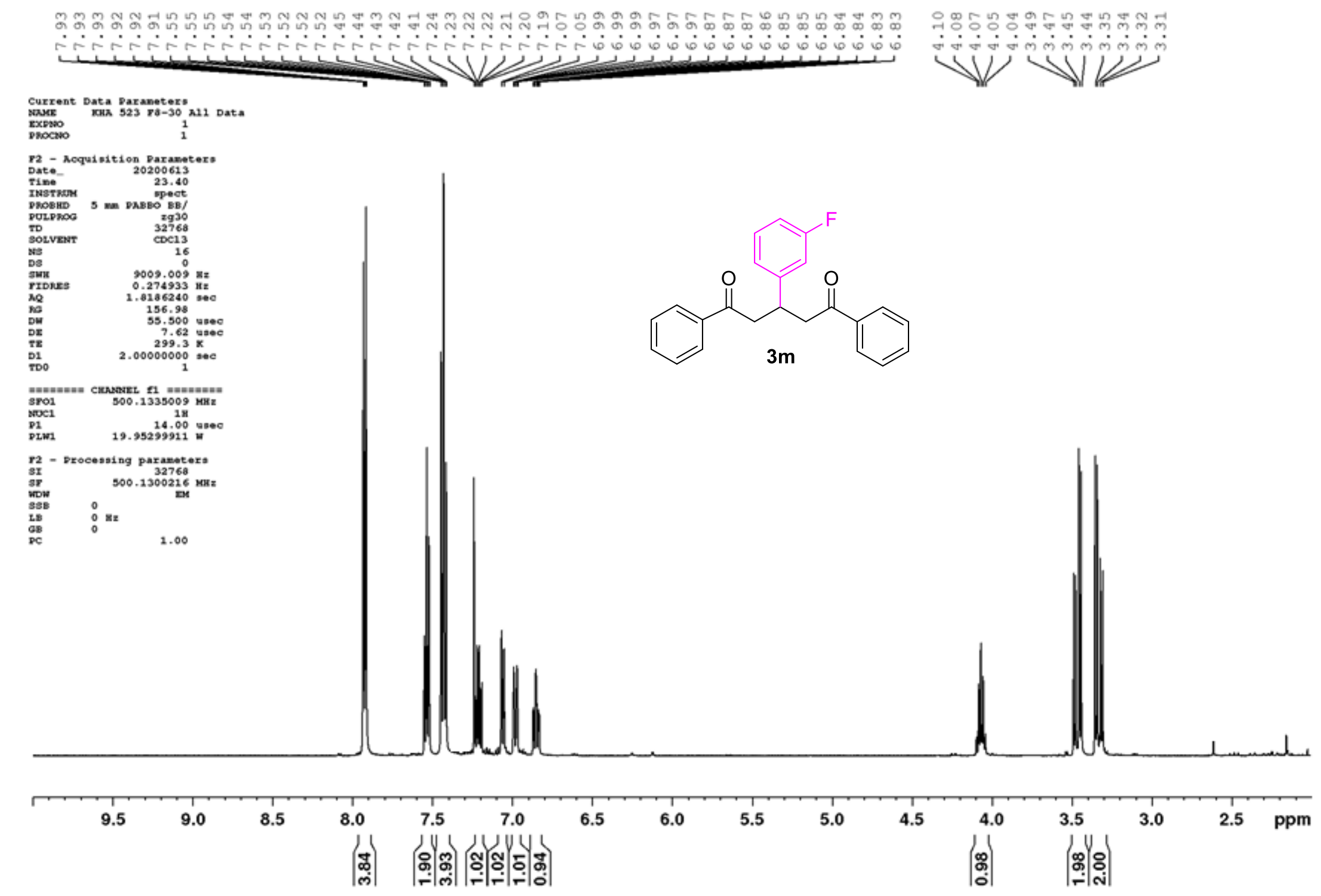

Figure S30. ${ }^{1} \mathrm{H}$ NMR spectrum of compound $3 \mathbf{m}\left(500 \mathrm{MHz}, \mathrm{CDCl}_{3}, 25{ }^{\circ} \mathrm{C}\right)$ 


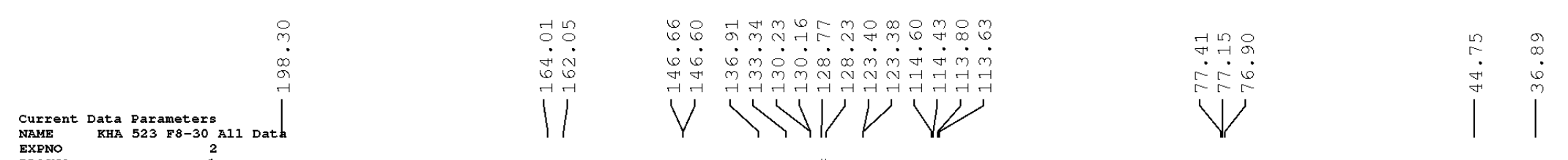
PROCNO F2 - Acquisition Parameters
Date_$\begin{array}{lr}20200614 \\ \text { Time } & 5.38\end{array}$ INSTRUM
PROBHD $5 \mathrm{~mm}$ PAEBO BE/ $\begin{array}{ll}\text { PULPROG } & \text { zgpg30 } \\ \text { TD } & \text { TS612 }\end{array}$

$\begin{array}{lr}\text { SOLVENT } & \begin{array}{l}34612 \\ \text { NS }\end{array} \\ \text { NS13 } & 8000\end{array}$

$\begin{array}{ll}\text { DS } & 8000 \\ \text { SWH } & 28846.154 \mathrm{~Hz} \\ \text { FIDRES } & 0.833415 \mathrm{~Hz}\end{array}$

$\begin{array}{lc}\text { FIDRES } & 0.833415 \mathrm{~Hz} \\ \text { AQ } & 0.5999413 \mathrm{sec} \\ \text { RG } & 200.35 \\ D W & 17.333-4 s e \\ D E & 6.50 \mathrm{usec} \\ \mathrm{DE} & 299.3 \mathrm{~K}\end{array}$

$\begin{array}{lr}\mathrm{DE} & 6.50 \mathrm{usc} \\ \mathrm{TE} & 299.3 \mathrm{k} \\ \mathrm{D} 1 & 2.0000000 \mathrm{sec} \\ \mathrm{D} 11 & 0.0300000 \mathrm{sec}\end{array}$

DDO $\quad 0.03000000$ sec

$=======$ CHANNEI $f 1$ = $======$
SFO1
125.7716219 MHz

$\begin{array}{lc}\text { NUC1 } & 13 \mathrm{C} \\ \text { P1 } & 10.75 \text { usec } \\ \text { PLW1 } & 50.11899948 \mathrm{w}\end{array}$

$=======$
SFO2

SFO2 500.1320005 MHE

$\begin{array}{lc}\text { NUC2 } & \text { 1H } \\ \text { CDPRRG [2 } & \text { waltz16 } \\ \text { PCPD2 } & 80.00 \text { us }\end{array}$

$\begin{array}{ll}\text { PCPD2 } & 80.00 \text { use } \\ \text { PLW2 } & 19.95299911 \mathrm{w}\end{array}$

\begin{tabular}{lr} 
PIW2 & $19.95299911 \mathrm{~W}$ \\
PLW12 & $0.63826001 \mathrm{~W}$ \\
\hline
\end{tabular}

F2 - Processing parameters

SI 32768

$\begin{array}{ll}\text { SF } & 125.7577738 \mathrm{MH}\end{array}$

$\begin{array}{lll}\mathrm{SSB} & 0 & 2.00 \mathrm{~Hz} \\ \mathrm{IE} & 0 & \\ \mathrm{~GB} & 0 & \end{array}$
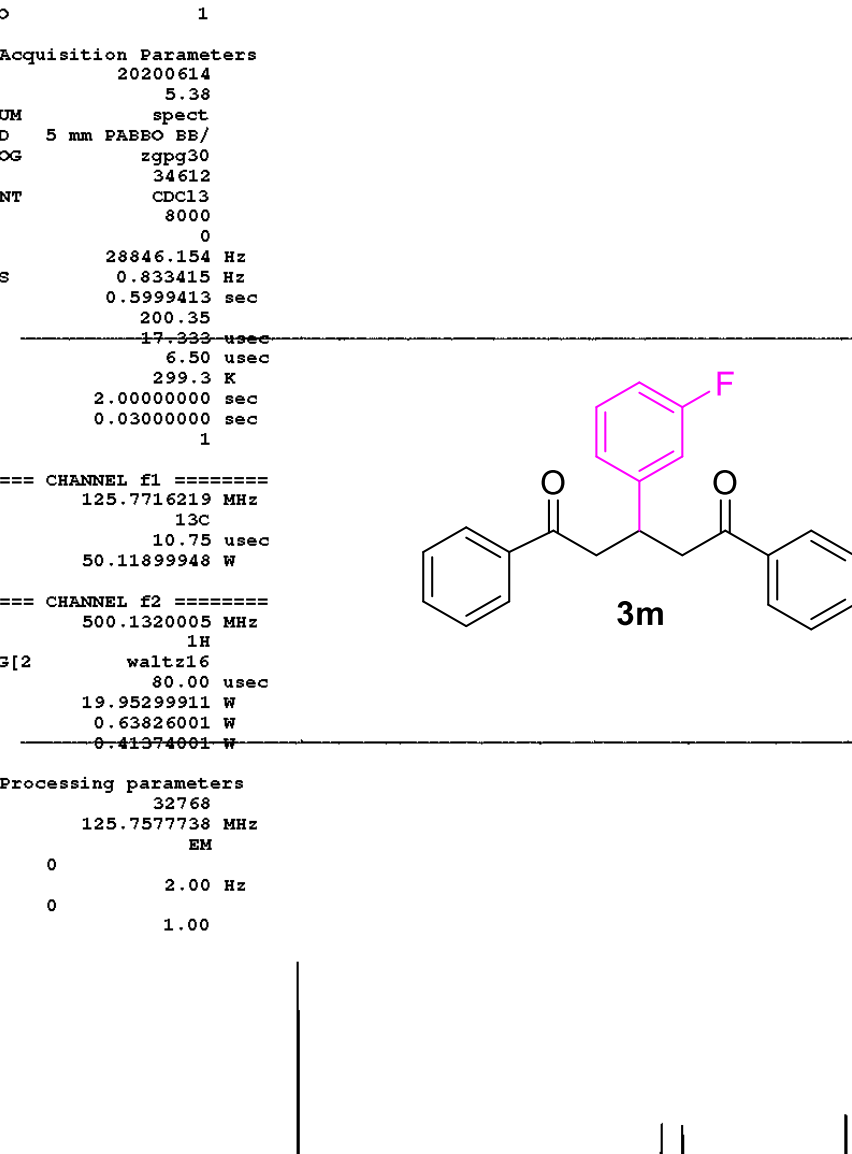

DEPT 135
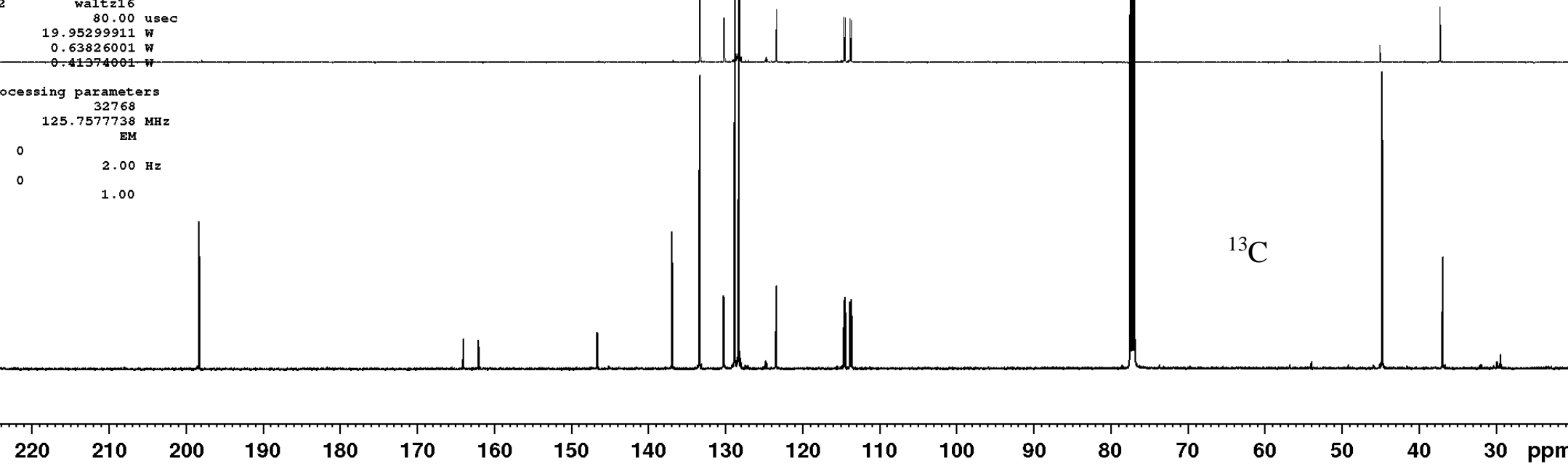

120

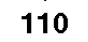

80

70

60

50

40

30 ppm

Figure S31. ${ }^{13} \mathrm{C}$ NMR spectrum of compound $3 \mathbf{m}\left(125 \mathrm{MHz}, \mathrm{CDCl}_{3}, 25{ }^{\circ} \mathrm{C}\right)$ 


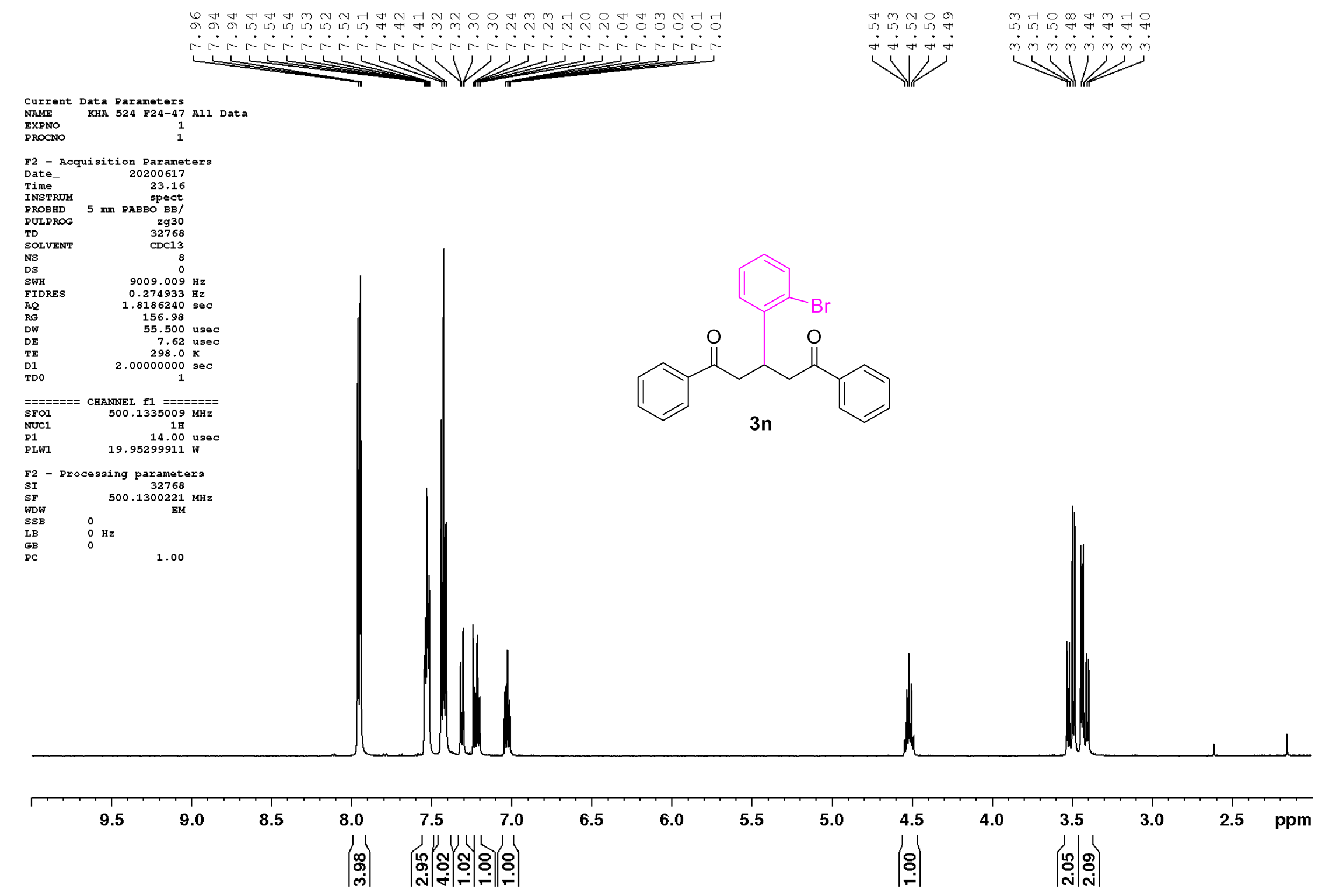

Figure S32. ${ }^{1} \mathrm{H}$ NMR spectrum of compound 3n (500 $\left.\mathrm{MHz}, \mathrm{CDCl}_{3}, 25{ }^{\circ} \mathrm{C}\right)$ 


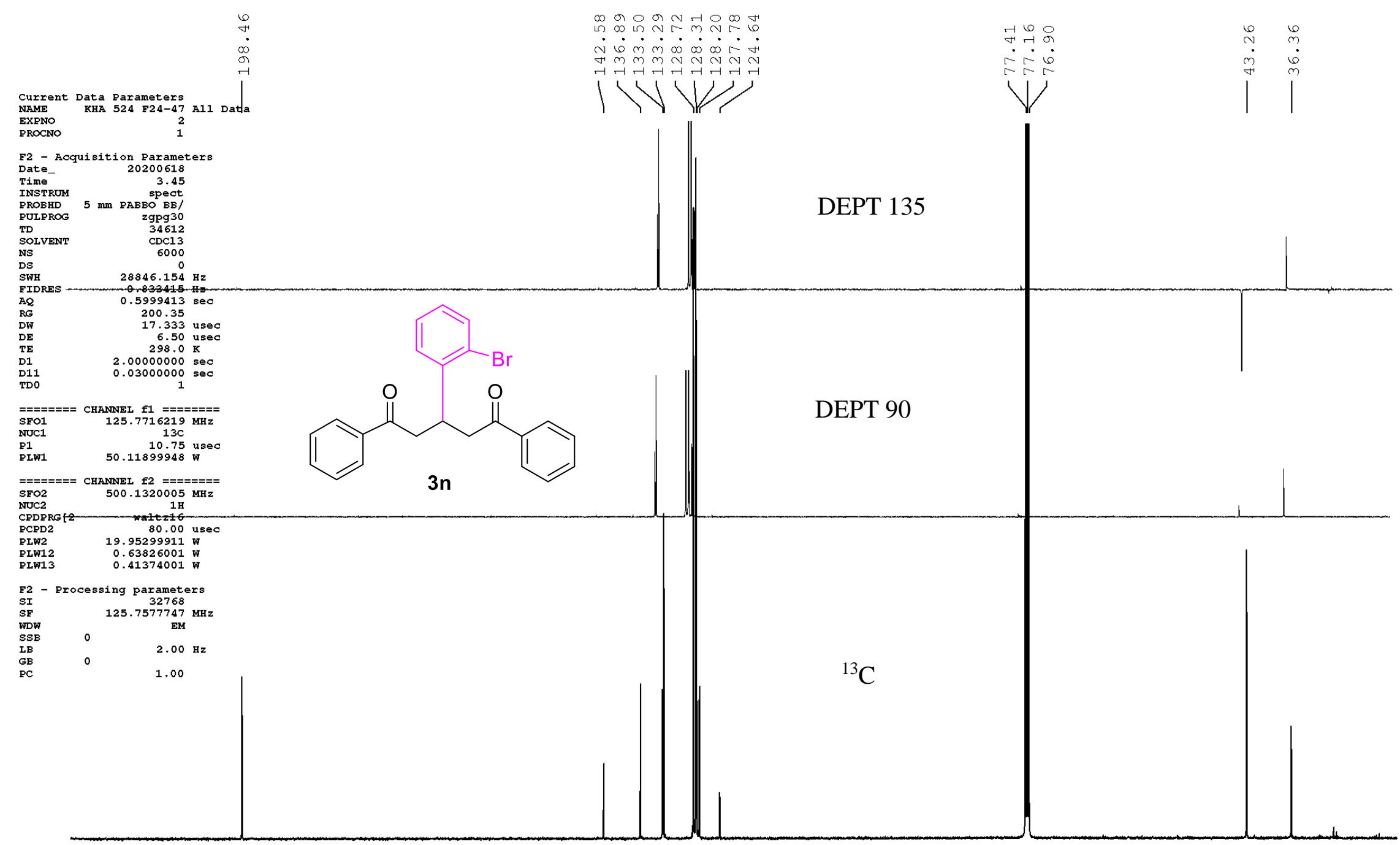

22

180

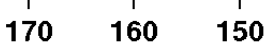

140

130

120

$110 \quad 100$

90

70

60

$50 \quad 40$

30 ppm

Figure S33. ${ }^{13} \mathrm{C}$ NMR spectrum of compound 3n $\left(125 \mathrm{MHz}, \mathrm{CDCl}_{3}, 25{ }^{\circ} \mathrm{C}\right)$ 


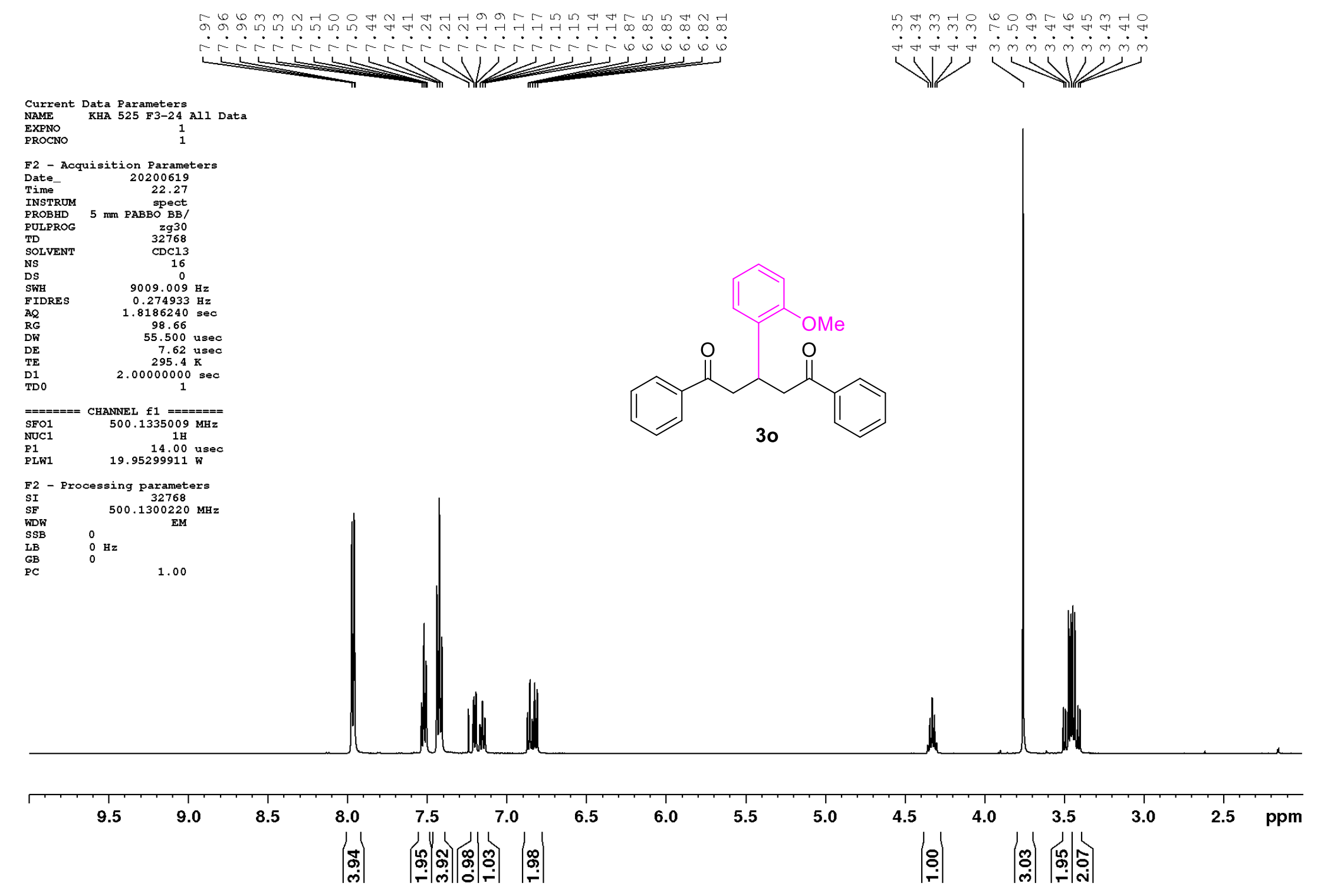

Figure S34. ${ }^{1} \mathrm{H}$ NMR spectrum of compound $30\left(500 \mathrm{MHz}, \mathrm{CDCl}_{3}, 25{ }^{\circ} \mathrm{C}\right)$ 


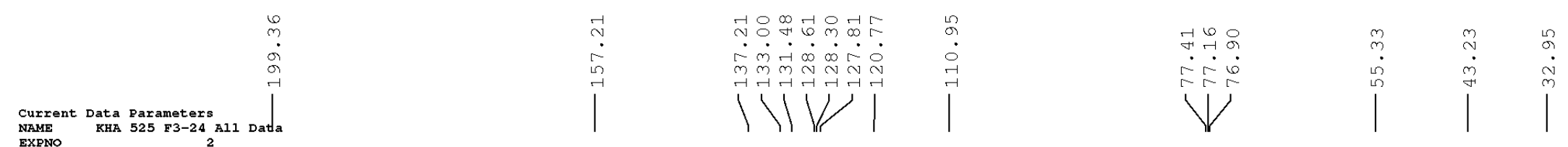

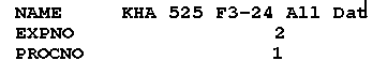

F2 - Acguisition 1

F2 - Acquisition Parameters

20200620

INSTRUM

PROBHD $5 \mathrm{~mm}$ PABEO BE/
PULPROG

TD

$\begin{array}{lc}\text { DS } & 0 \\ \text { SWH } & 28846.154 \mathrm{~Hz} \\ \text { FIDRES } & 0.833415 \mathrm{~Hz} \\ \text { AQ } & 0.5999413 \mathrm{sec} \\ \text { RG } & 200.35 \\ \text { DW } & 17.333 \text { usec } \\ \text { DW } & \end{array}$

$\begin{array}{rr}\text { DW } & 200.35 \\ D E & 17.333 \text { usec } \\ \text { DE } & 6.50 \text { usec }\end{array}$

$\begin{array}{lr}\text { TE } & 294.7 \mathrm{~K} \\ \mathrm{D} 1 & 2.00000000 \mathrm{sec}\end{array}$

TDO

$=======$ CHANNEL $f 1=======$
SFO1
NUC1
$125.7716219 \mathrm{MHz}$

$\begin{array}{lr}\text { P1 } & 13 \mathrm{C} \text { usec } \\ \text { PLW1 } & 50.11899948 \text { w }\end{array}$

$=======$
$S F O 2$

$\begin{array}{lr}\text { SFO2 } & 500.1320005 \\ \text { NUC2 } & 1 \mathrm{H} \\ \text { CPDPRG [2 } & \text { waltz16 }\end{array}$

PCPD2 $\quad 80.00$ use

PLW12 $\frac{19.95299911 \text { W }}{0.63826001}$

F2 - Processing parameters

SI 32768

WDW $\quad 125.7577765 \mathrm{M}$

$\begin{array}{lll}\text { SSB } & 0 & 2.00 \mathrm{~Hz} \\ \mathrm{IB} & 0 & \end{array}$

$\begin{array}{ll}0 & 2.00 \\ & 1.00\end{array}$

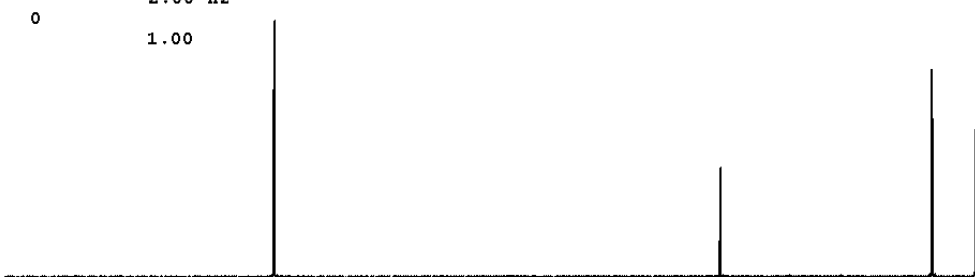

220

210200

190

180

160

150

140

130

120

110

100

70

60

50

30 ppm

Figure S35. ${ }^{13} \mathrm{C}$ NMR spectrum of compound $3 o\left(125 \mathrm{MHz}, \mathrm{CDCl}_{3}, 25{ }^{\circ} \mathrm{C}\right)$ 
Current Data Parameters
NAME
EXPNO
KHA 528 F6-36 A1l Data
8

\section{PROCNO}

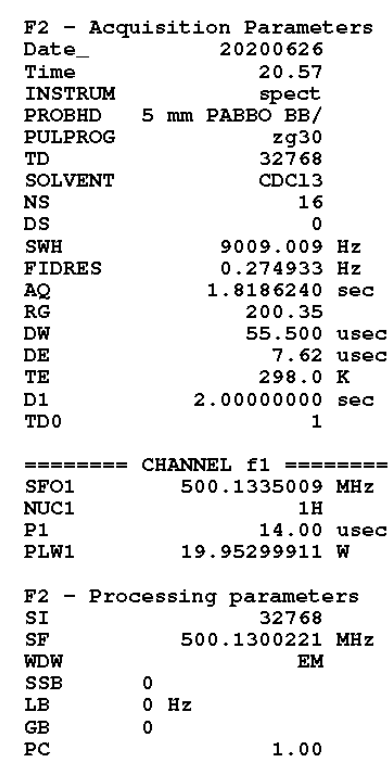
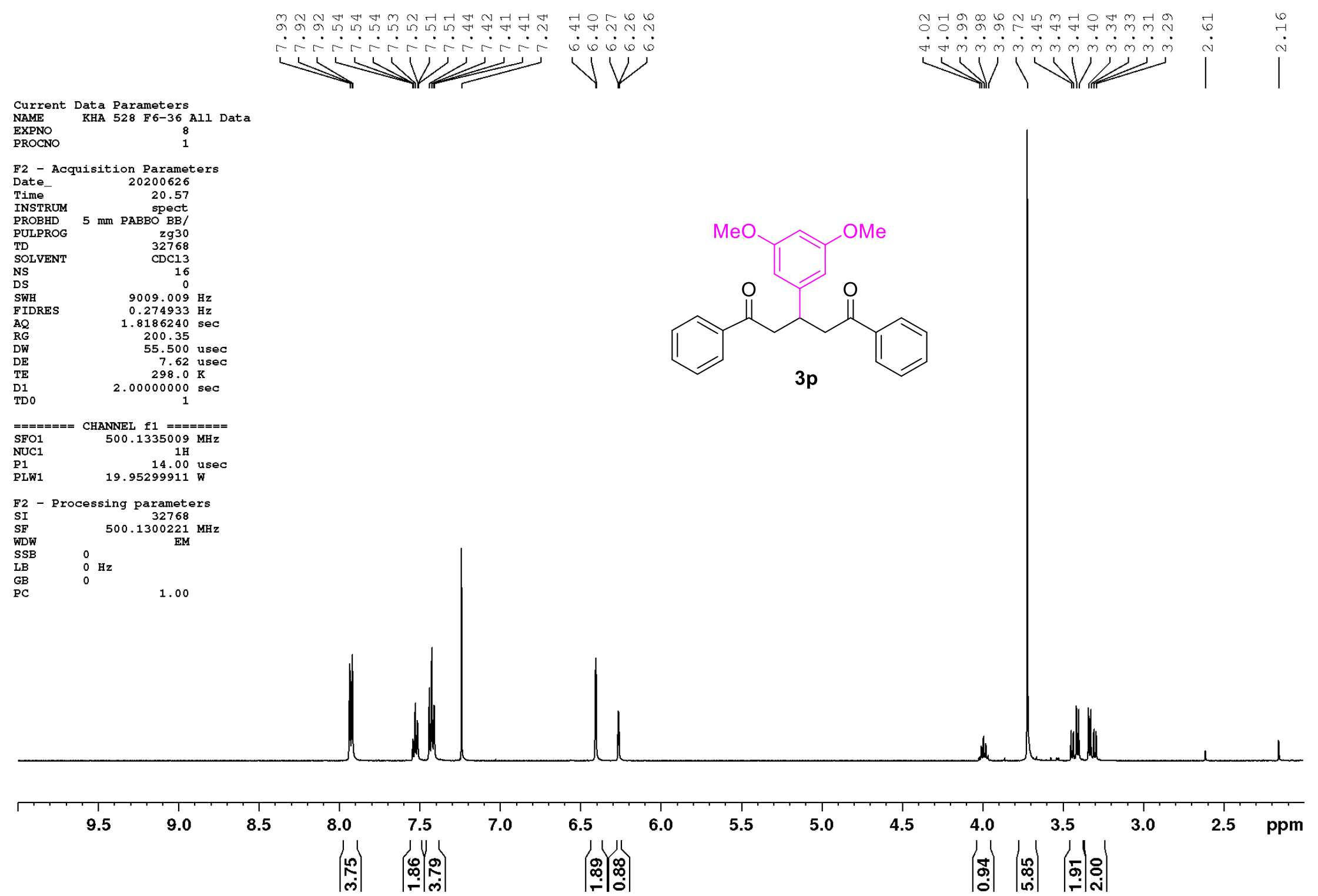

Figure S36. ${ }^{1} \mathrm{H}$ NMR spectrum of compound 3p $\left(500 \mathrm{MHz}, \mathrm{CDCl}_{3}, 25{ }^{\circ} \mathrm{C}\right)$ 


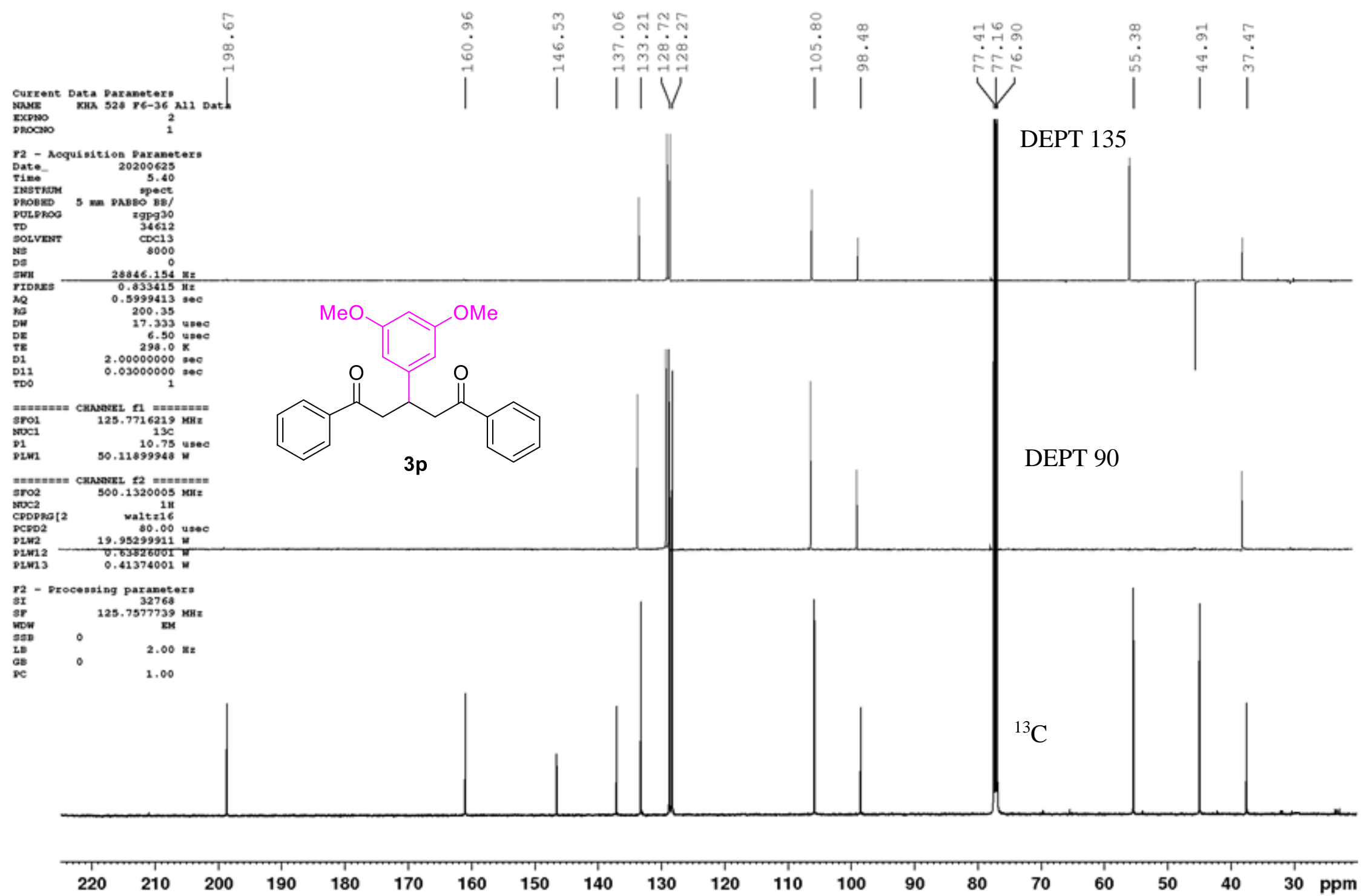

Figure S37. ${ }^{13} \mathrm{C}$ NMR spectrum of compound $3 \mathbf{p}\left(125 \mathrm{MHz}, \mathrm{CDCl}_{3}, 25{ }^{\circ} \mathrm{C}\right)$ 
Current Data Parameters
NAME
EXPNO
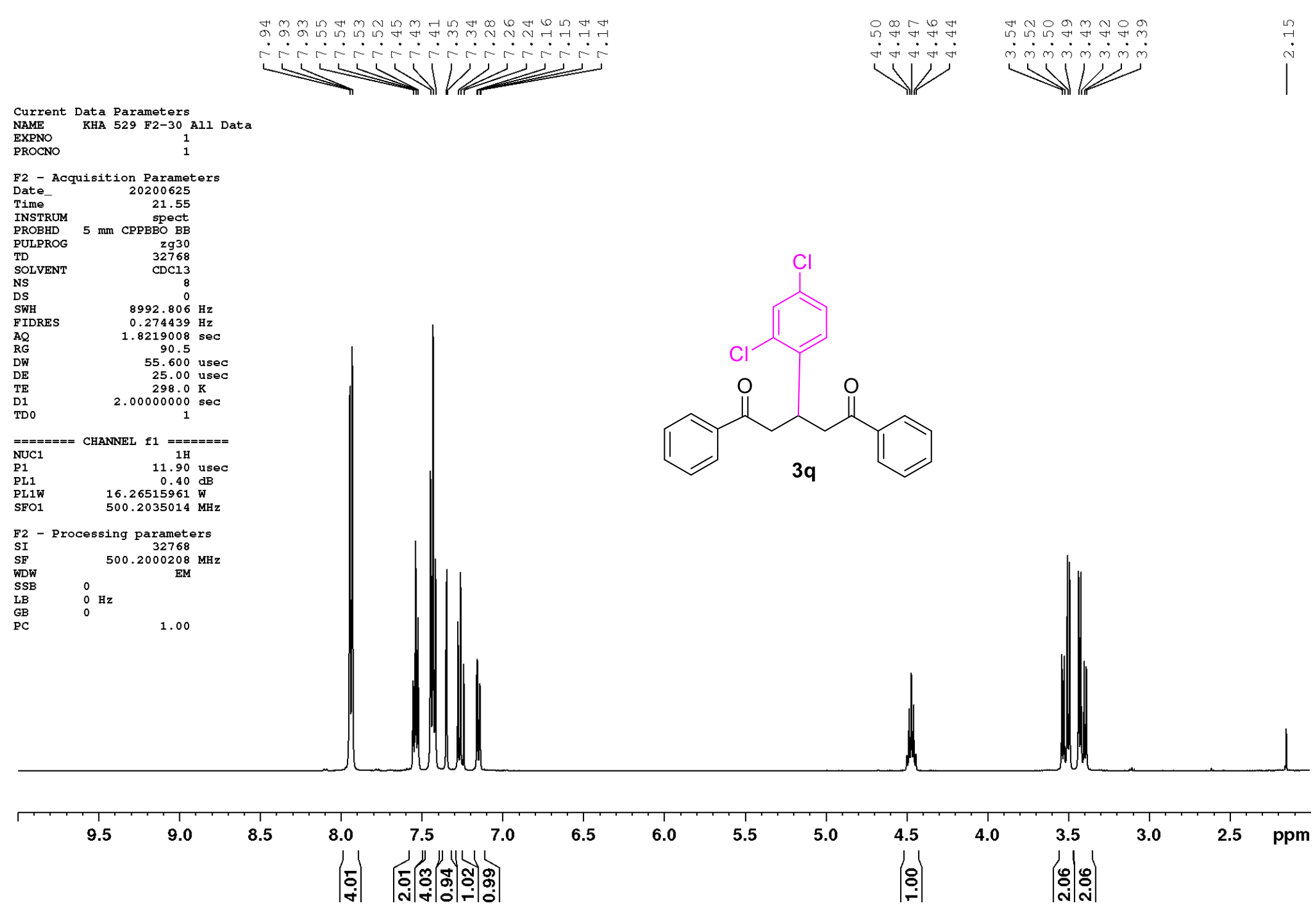

Figure S38. ${ }^{1} \mathrm{H}$ NMR spectrum of compound 3q $\left(500 \mathrm{MHz}, \mathrm{CDCl}_{3}, 25{ }^{\circ} \mathrm{C}\right)$ 

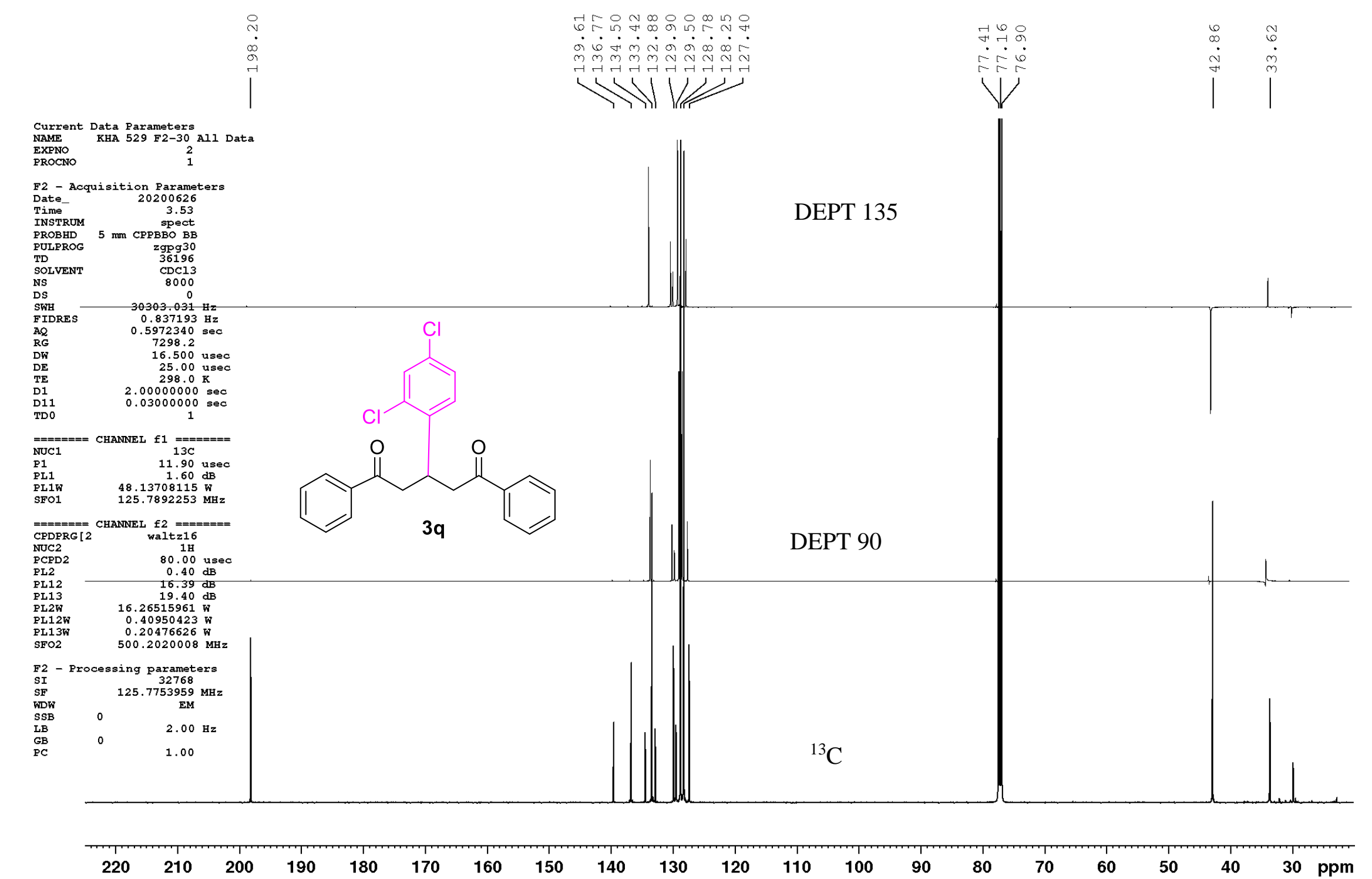

Figure S39. ${ }^{13} \mathrm{C}$ NMR spectrum of compound $\mathbf{3 q}\left(125 \mathrm{MHz}, \mathrm{CDCl}_{3}, 25{ }^{\circ} \mathrm{C}\right)$ 

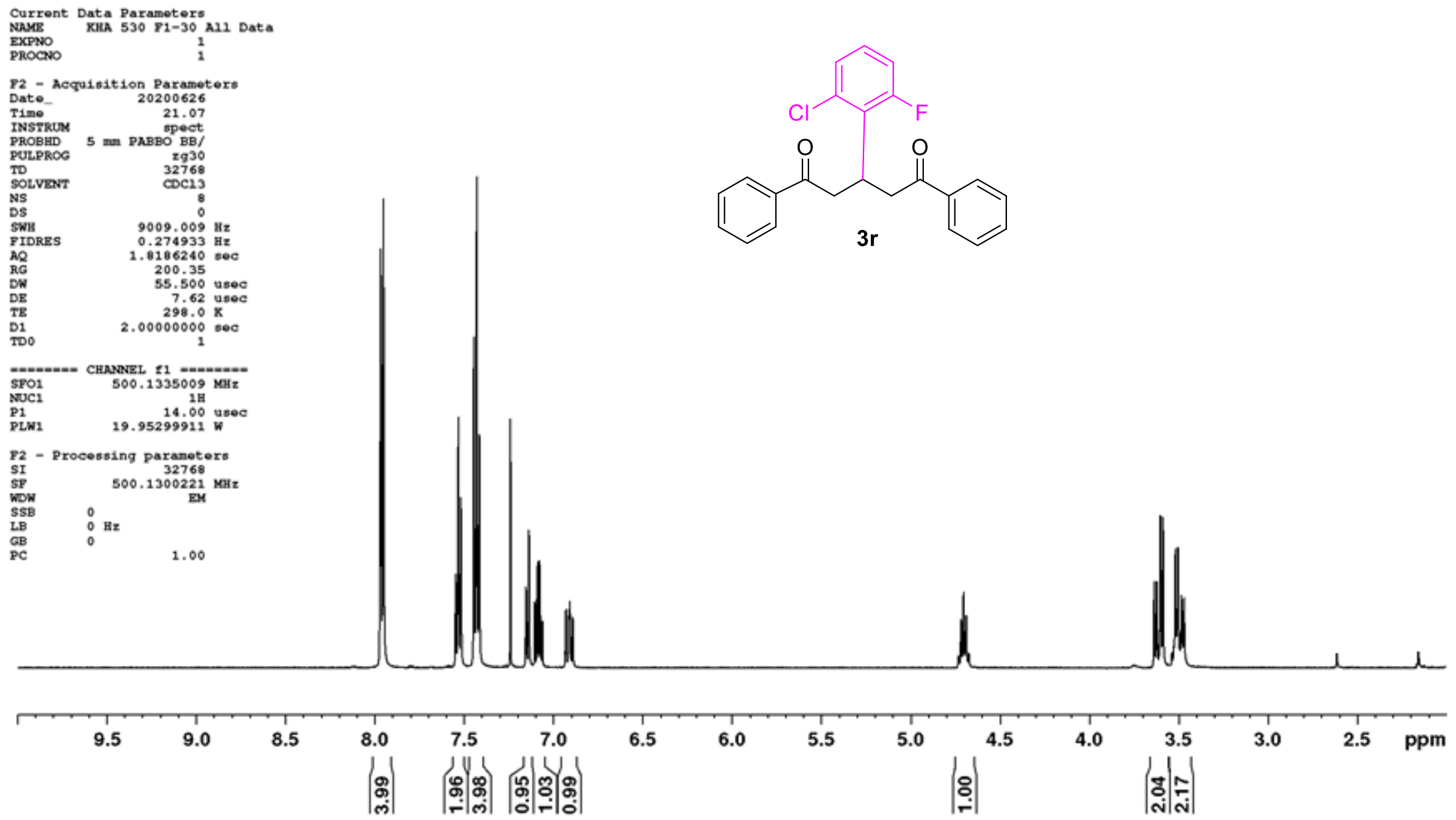

Figure S40. ${ }^{1} \mathrm{H}$ NMR spectrum of compound $3 \mathbf{r}\left(500 \mathrm{MHz}, \mathrm{CDCl}_{3}, 25{ }^{\circ} \mathrm{C}\right)$ 


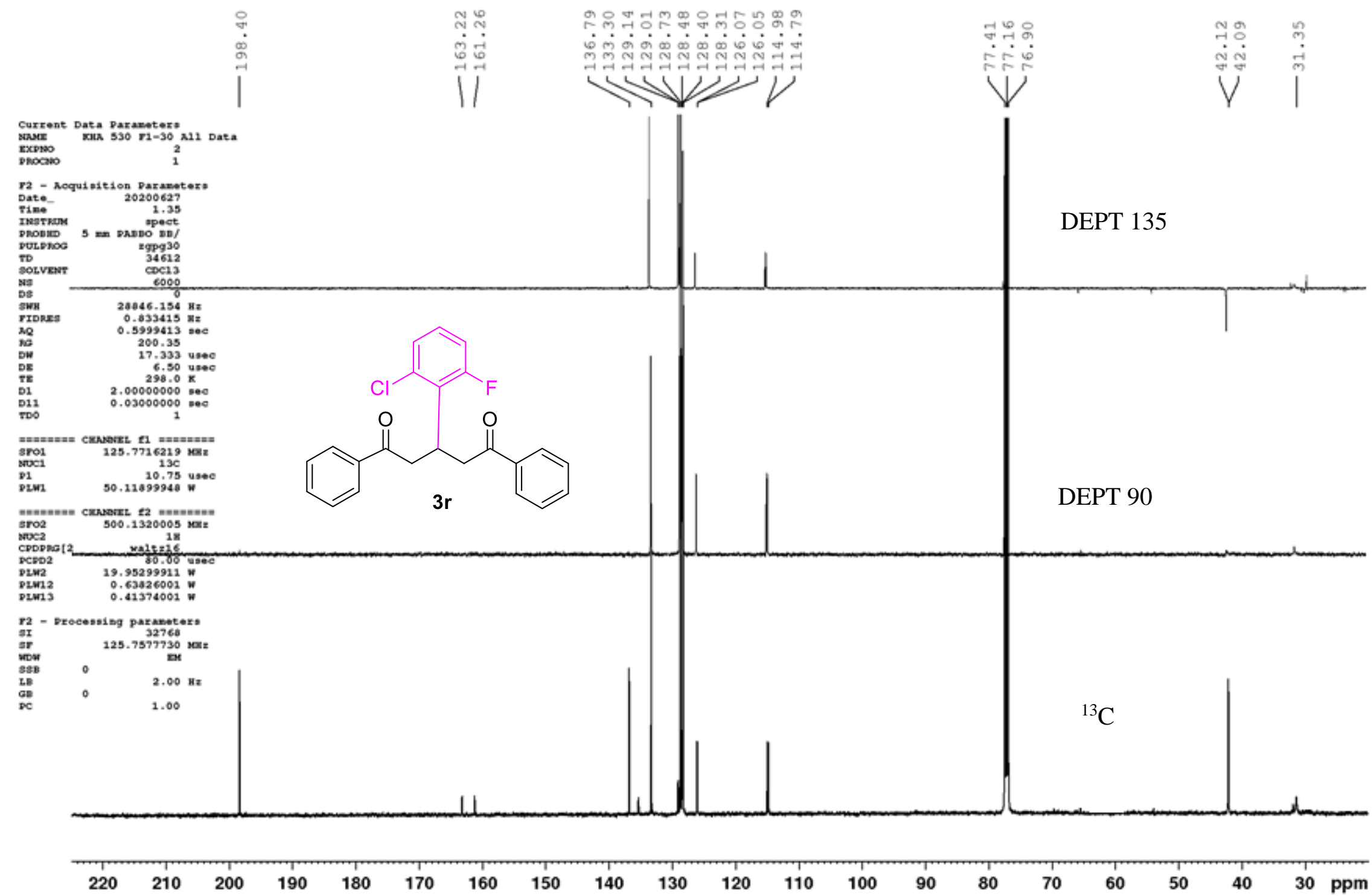

Figure S41. ${ }^{13} \mathrm{C}$ NMR spectrum of compound $3 \mathbf{r}\left(125 \mathrm{MHz}, \mathrm{CDCl}_{3}, 25{ }^{\circ} \mathrm{C}\right)$ 
Current Data Parameters
NaME KHA 558 F1-33 A11 Data

NQME
EXPNO
PROCNO

Timo 202008

PRSTRD $s$ m PABDO $\mathrm{spect}$

PULPROG

SOLVENT

$\begin{array}{ll}\text { NS } & 16 \\ \text { DSH } & 9009.009 \\ \text { PIDRES } & 0.274933 \text { 目 }\end{array}$

AQ $\quad 1.8186240 \mathrm{sec}$

$\begin{array}{lr}\text { RG } & 135.71 \\ \text { DW } & 55.500 \\ & \end{array}$

7.62 usec
$298.0 \mathrm{~K}$

DD $2.00000000 \mathrm{sec}$

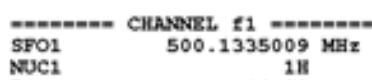

P1 $\quad 14.00$ use

F2 - Processing parameters

$\begin{array}{ll}\text { SI } & 32768 \\ \text { SF } & 500.1300213 \\ \text { MHz }\end{array}$

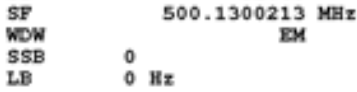

$\begin{array}{lll}\mathrm{LB} & 0 \mathrm{~Hz} & \\ \mathrm{~GB} & 0 & \\ \mathrm{PC} & & 1.00\end{array}$
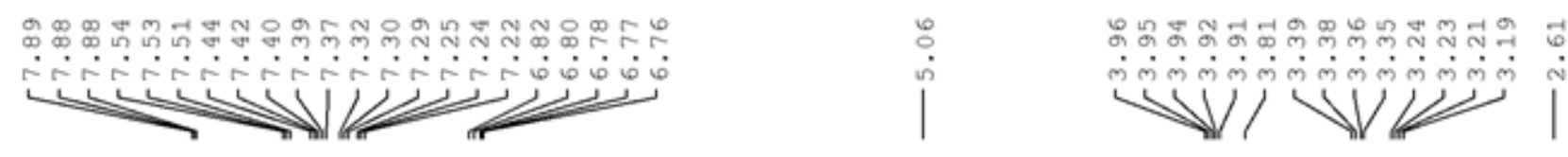

$(1.00$
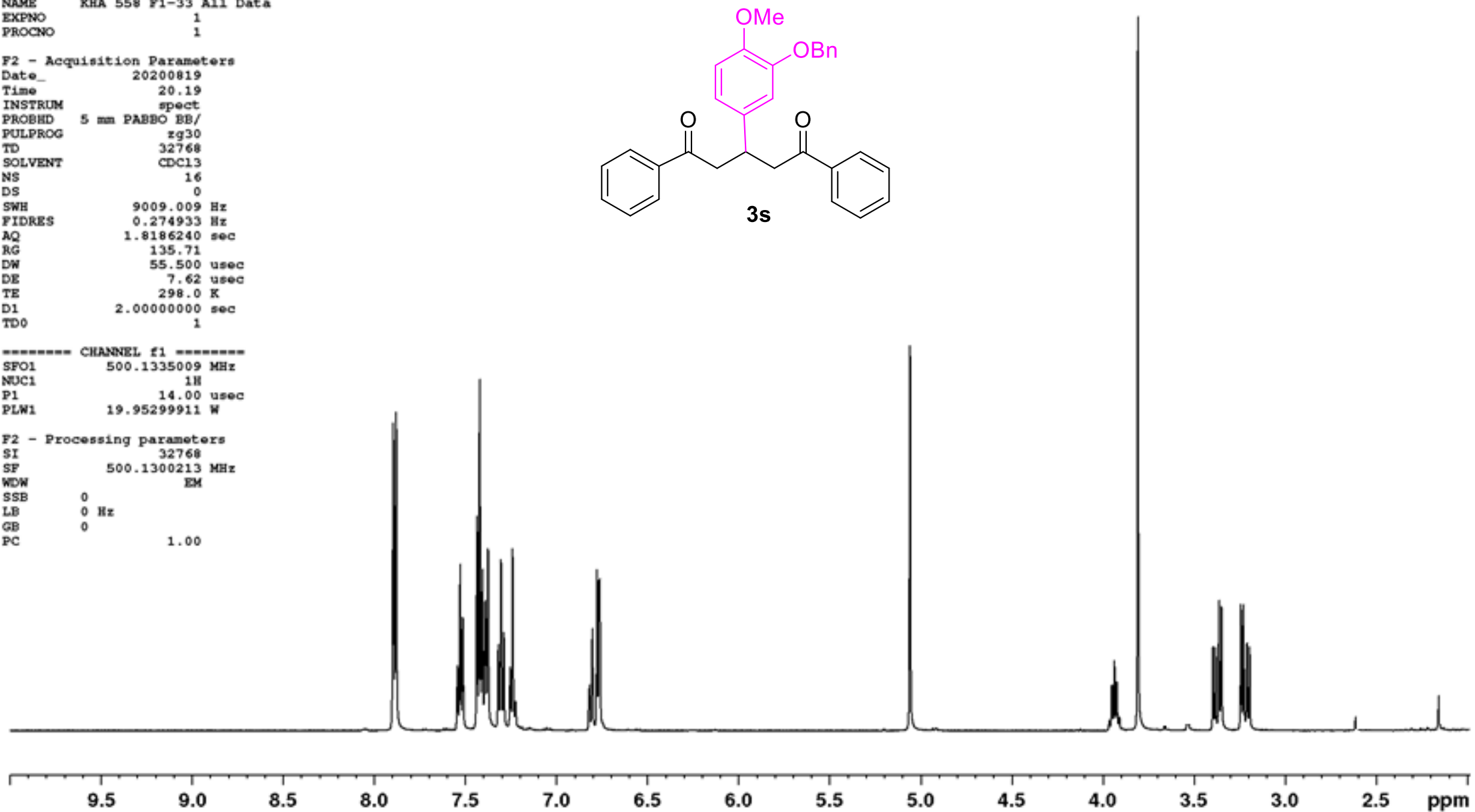

7.0

|

6.5
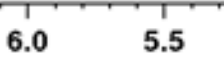

|

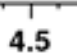

4.0

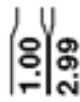

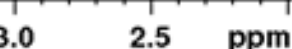

Figure S42. ${ }^{1} \mathrm{H}$ NMR spectrum of compound $3 \mathbf{s}\left(500 \mathrm{MHz}, \mathrm{CDCl}_{3}, 25{ }^{\circ} \mathrm{C}\right)$ 


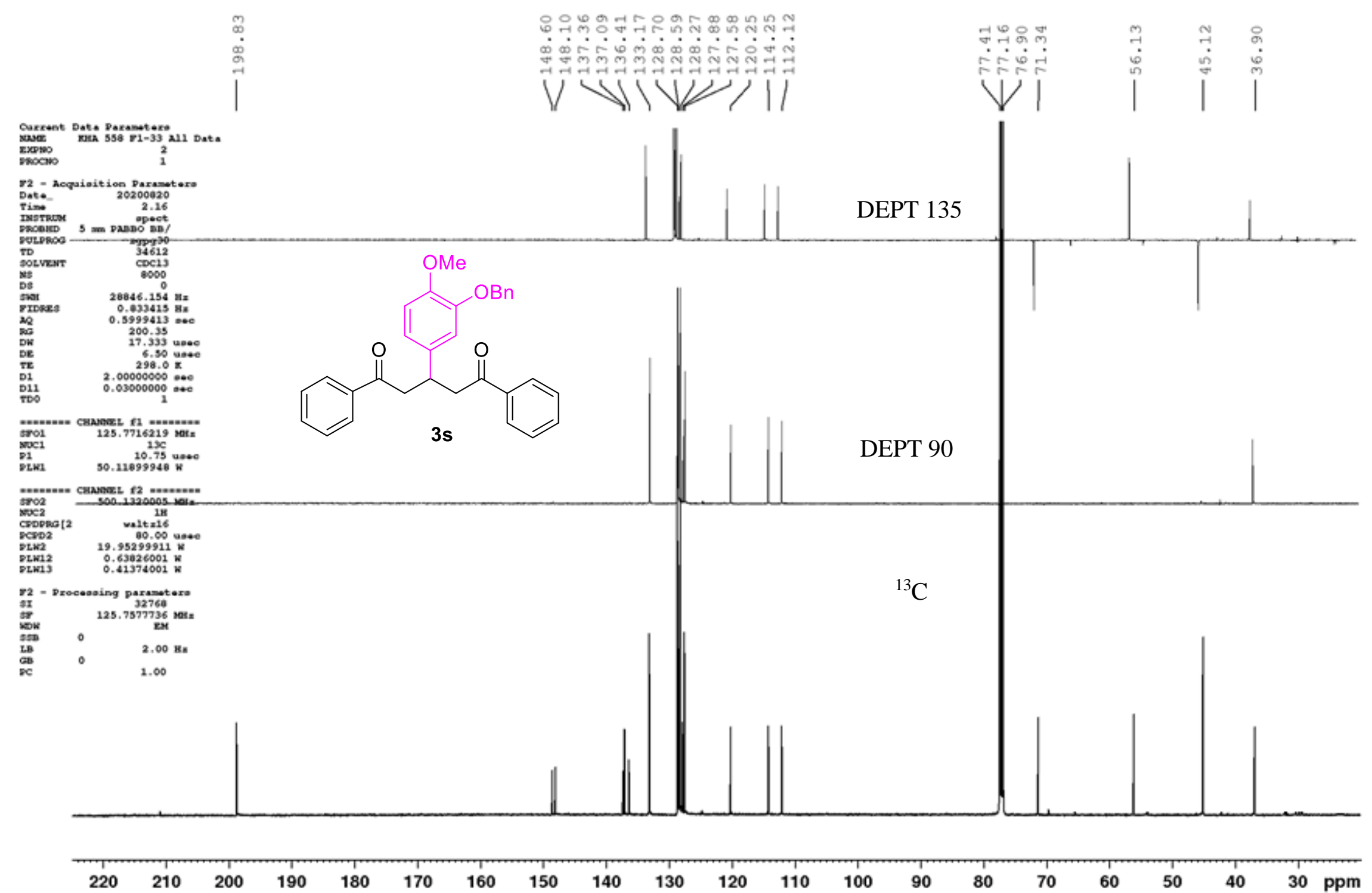

Figure S43. ${ }^{13} \mathrm{C}$ NMR spectrum of compound 3s $\left(125 \mathrm{MHz}, \mathrm{CDCl}_{3}, 25{ }^{\circ} \mathrm{C}\right)$ 

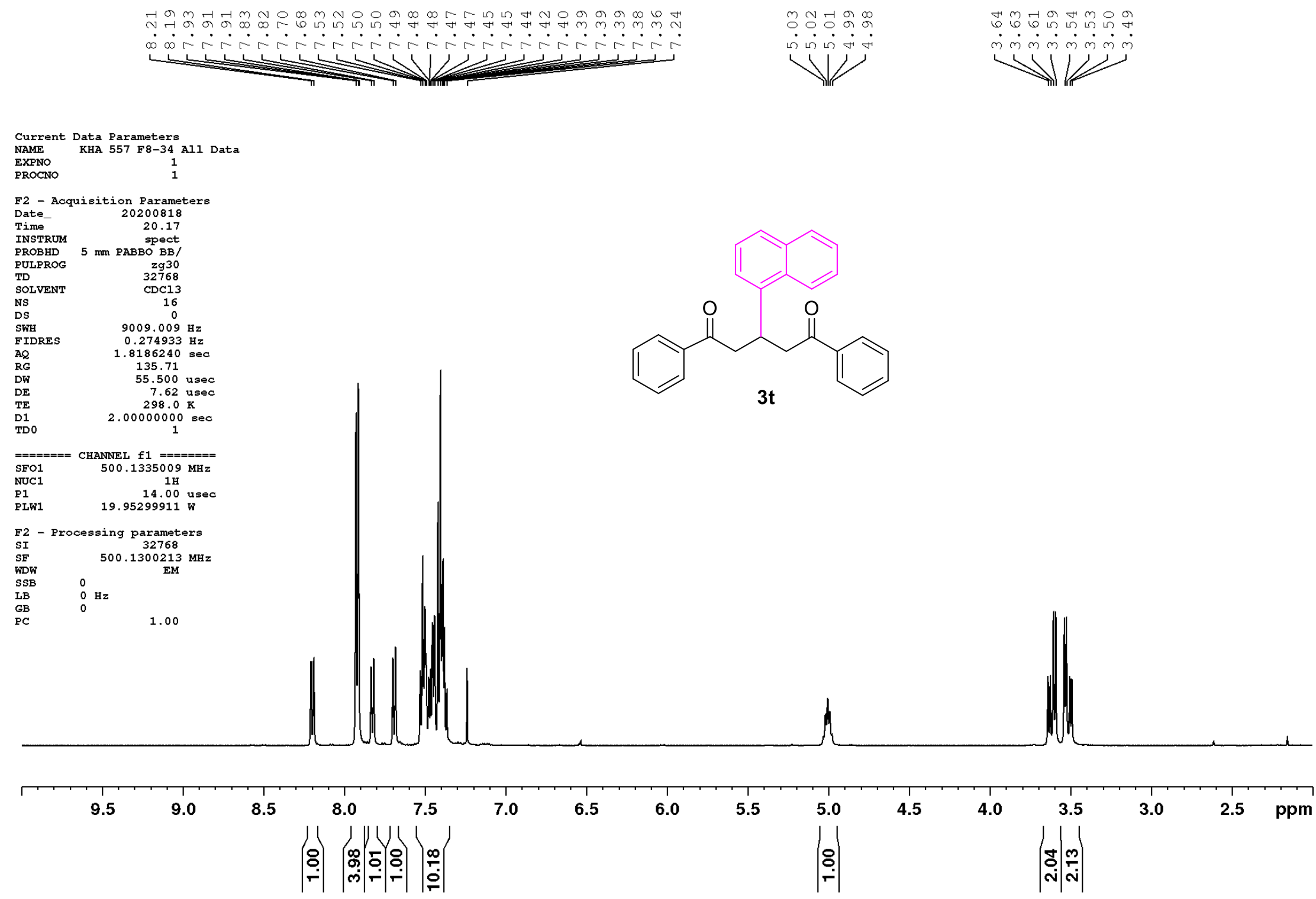

Figure S44. ${ }^{1} \mathrm{H} \mathrm{NMR}$ spectrum of compound $3 \mathrm{t}\left(500 \mathrm{MHz}, \mathrm{CDCl}_{3}, 25{ }^{\circ} \mathrm{C}\right)$ 
$\underset{\text { CAMrent Data Parameters }}{\text { KHA } 557 \text { F8-34 A11 Data }}$

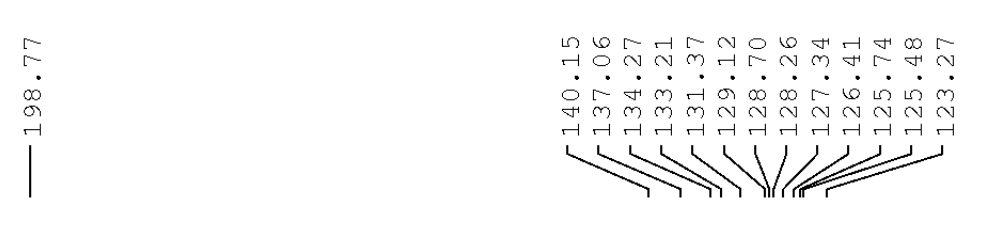

DEPT 135

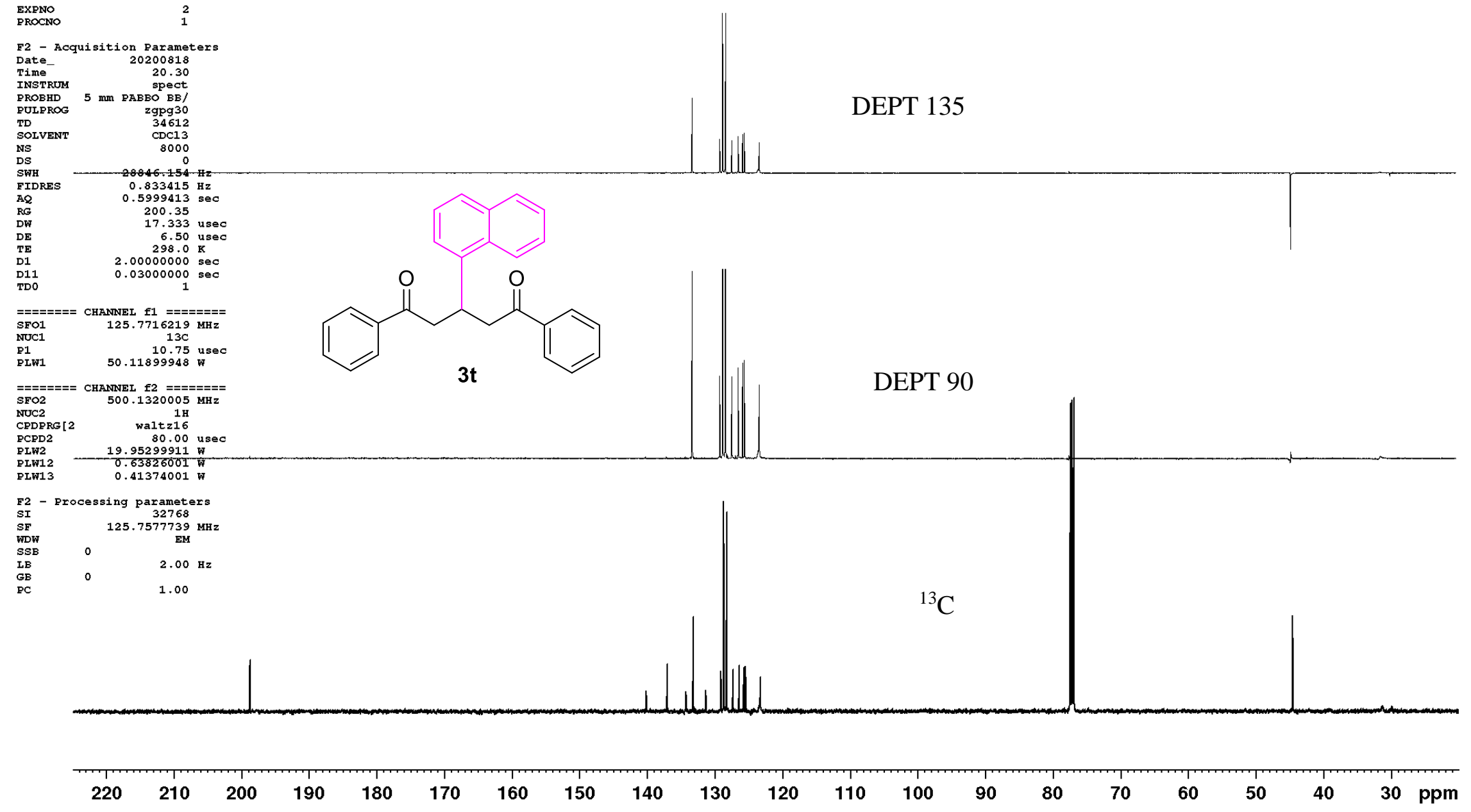

Figure S45. ${ }^{13} \mathrm{C}$ NMR spectrum of compound $3 \mathrm{t}\left(125 \mathrm{MHz}, \mathrm{CDCl}_{3}, 25{ }^{\circ} \mathrm{C}\right)$ 
Current Data Parameters

NeNGE KHA 534 F1-25 A11 Data

EXPNO

Y2 - Aequisition Paramete

Time-

PROBHD

PULPROC

To

NS

SWH

FIDR

AQ

DW

TE

1

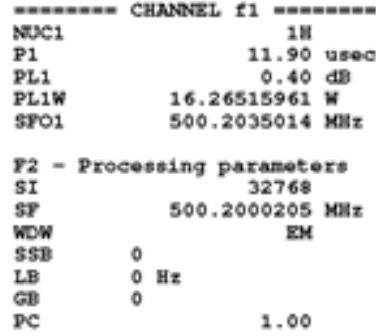

œ

vivivi

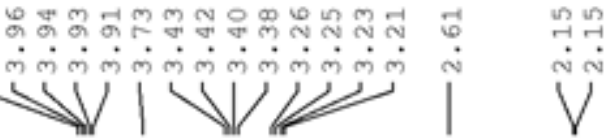

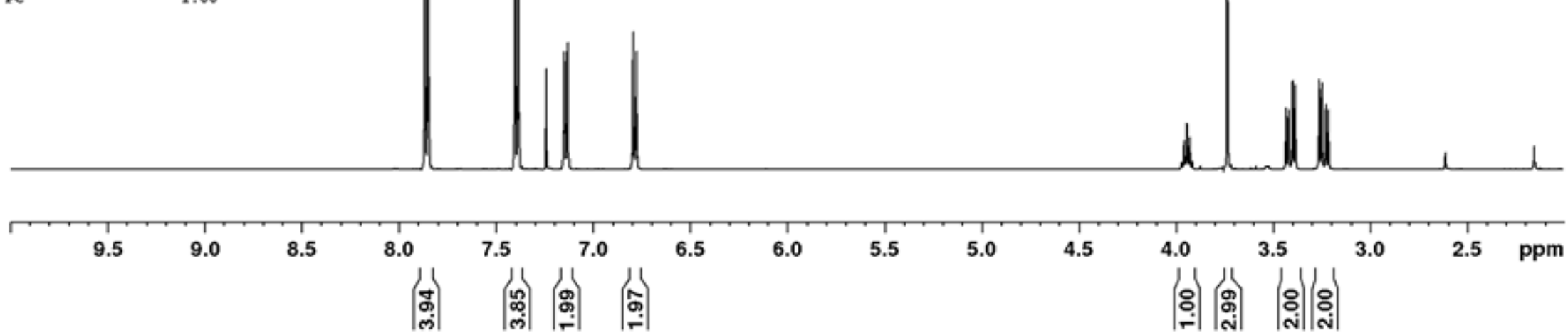

Figure S46. ${ }^{1} \mathrm{H} \mathrm{NMR}$ spectrum of compound $4 \mathbf{a}\left(500 \mathrm{MHz}, \mathrm{CDCl}_{3}, 25{ }^{\circ} \mathrm{C}\right)$ 


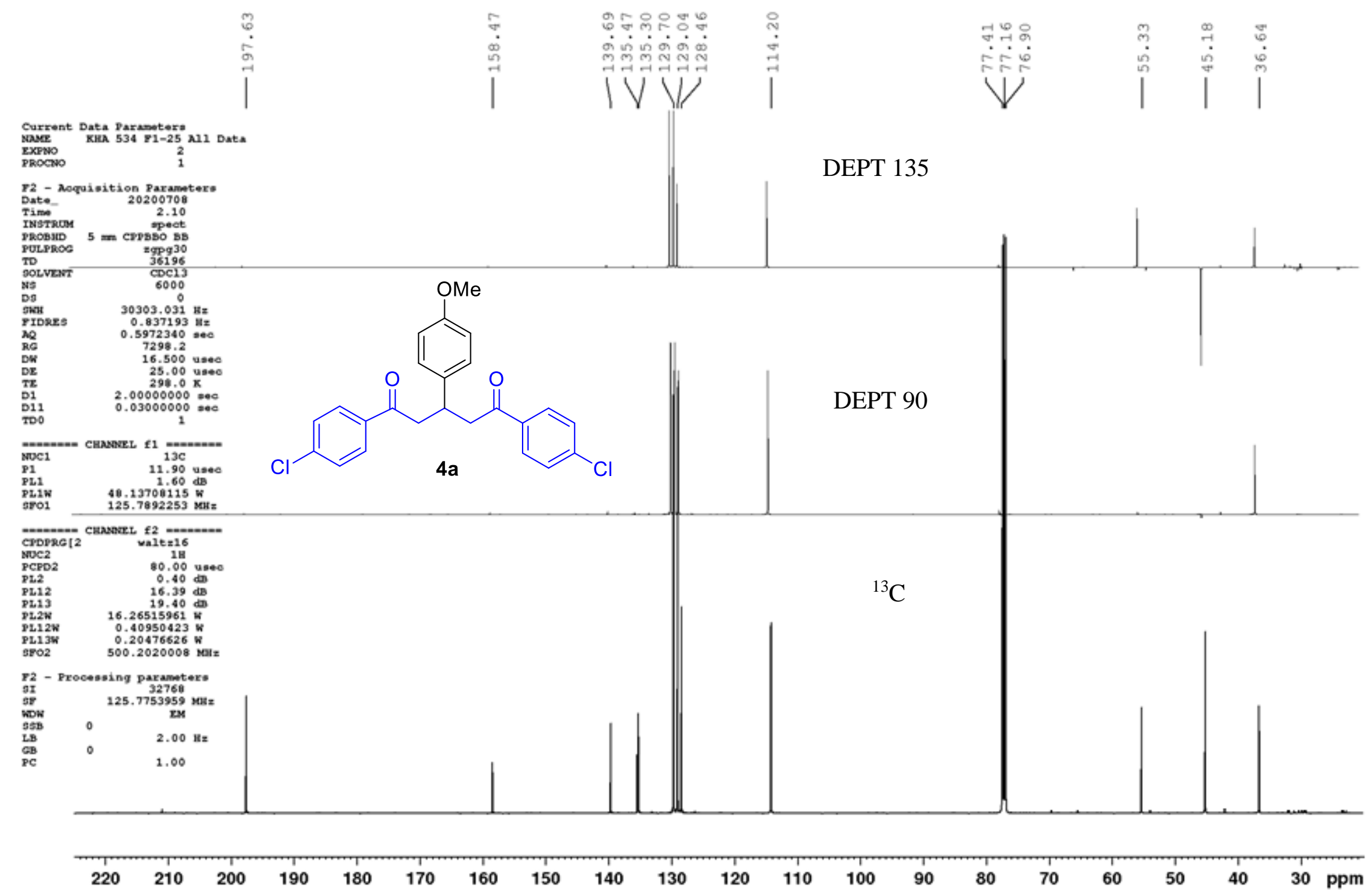

Figure S47. ${ }^{13} \mathrm{C}$ NMR spectrum of compound $4 \mathbf{a}\left(125 \mathrm{MHz}, \mathrm{CDCl}_{3}, 25{ }^{\circ} \mathrm{C}\right)$ 
Current Data Parameters
NAME KHA 533 F21-47 All Data
EXPNO
PROCNO
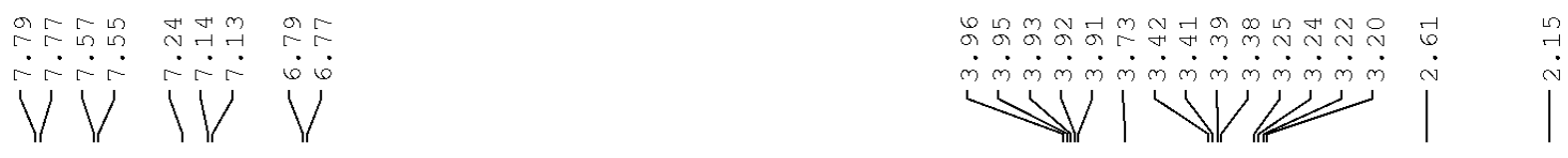

F2 - Acquisition Parameters

Date

INSTRUM 22.19

PROBHD $5 \mathrm{~mm}$ PABBO BB/

zg30

SOLVEN

DS

SWH 0

FIDRES $\quad 0.274933 \mathrm{~Hz}$

RG $\quad 1.8186240$

55.500 use

$\begin{array}{lr}\mathrm{DE} & 298.0 \mathrm{~K} \\ \mathrm{TE} & 2.00000000 \mathrm{sec}\end{array}$

TD0

$=======\quad \begin{gathered}\text { CHANNEL } \mathrm{f} 1 \mathrm{l}====== \\ 500.1335009 \mathrm{MHz}\end{gathered}$
SFO1

NUC1 $1 \mathrm{H}$

$\begin{array}{lr}\text { P1 } & 14.00 \text { usec } \\ \text { PLW1 } & 19.95299911 \mathrm{~W}\end{array}$

$\begin{array}{lr}\text { F2 } & \text { - Processing parameters } \\ \text { SI } & 32768 \\ \text { SF } & 500.1300221 \mathrm{MHz}\end{array}$

WF $\quad 500.1300221 \mathrm{MI}$

$\begin{array}{ll}\text { SSB } & 0 \\ \text { LB } & 0\end{array}$

$\begin{array}{lll}\text { GB } & 0 & 1.00\end{array}$

$\mid$

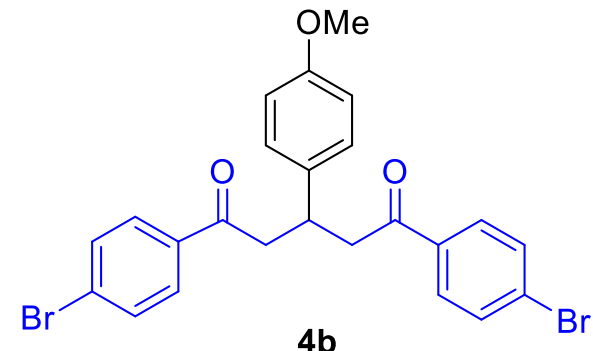

$4 b$
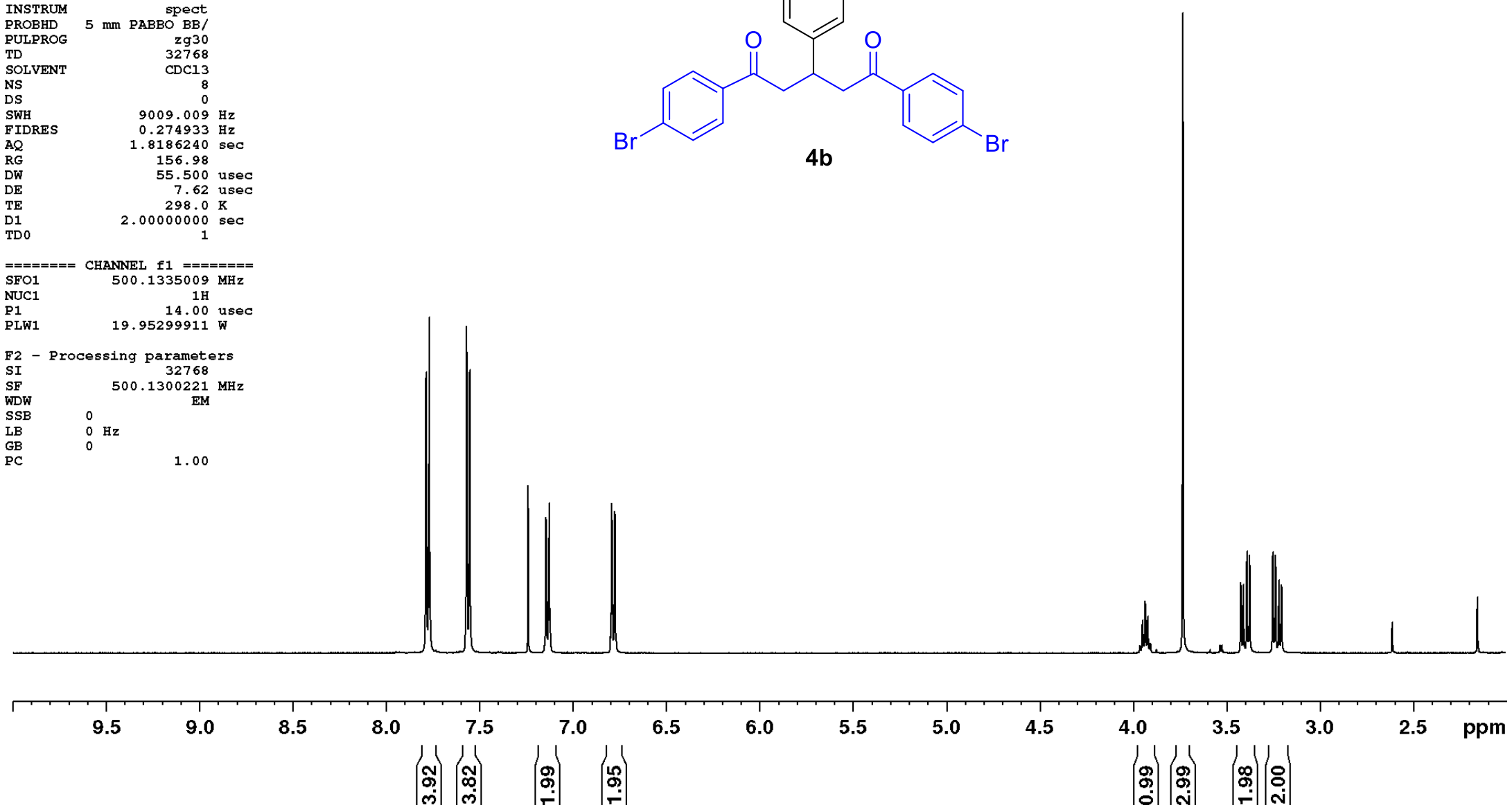

Figure S48. ${ }^{1} \mathrm{H}$ NMR spectrum of compound $4 \mathbf{b}\left(500 \mathrm{MHz}, \mathrm{CDCl}_{3}, 25{ }^{\circ} \mathrm{C}\right)$ 
Current Data Parameters
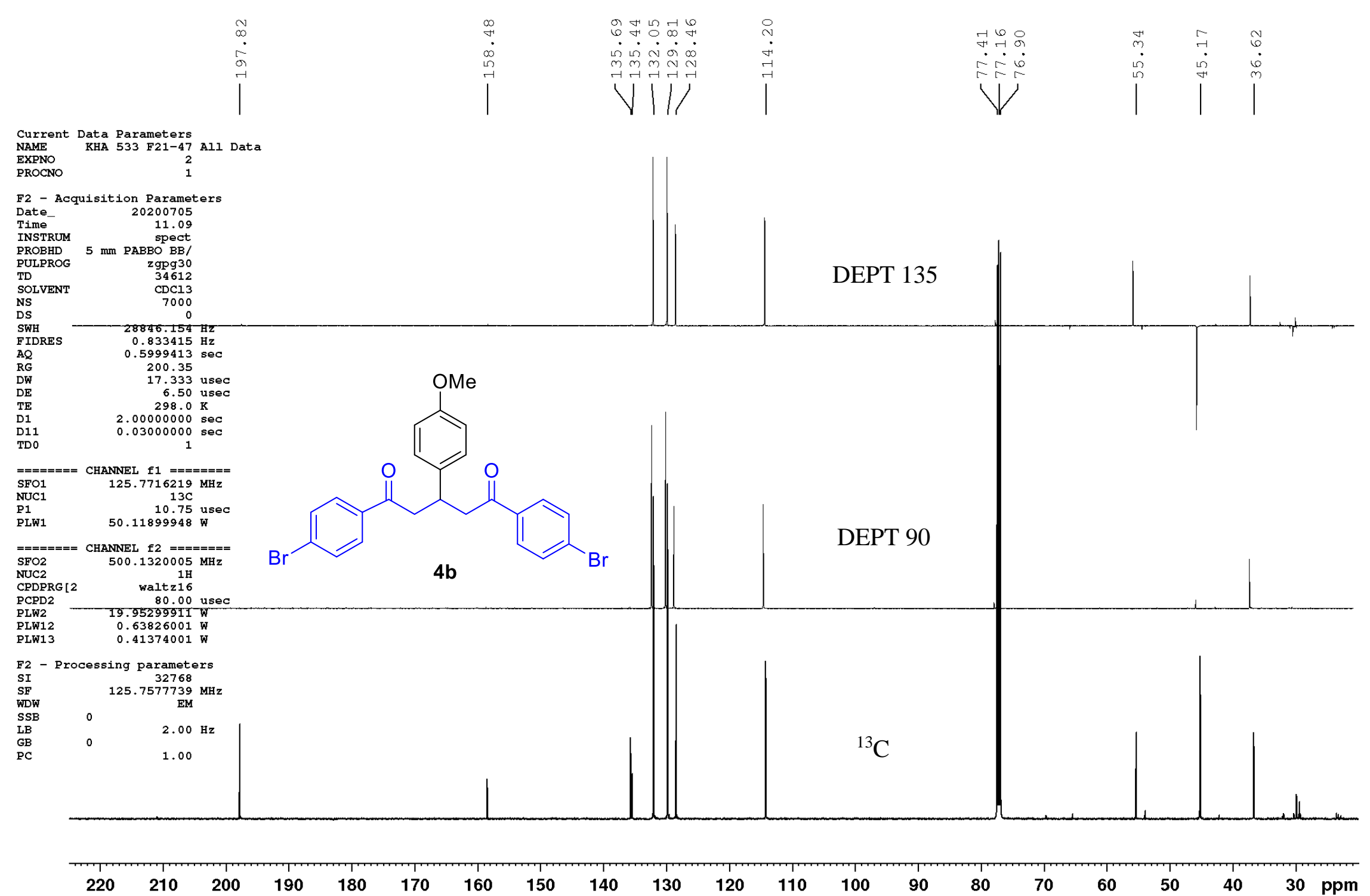

Figure S49. ${ }^{13} \mathrm{C}$ NMR spectrum of compound $4 \mathbf{b}\left(125 \mathrm{MHz}, \mathrm{CDCl}_{3}, 25{ }^{\circ} \mathrm{C}\right)$ 


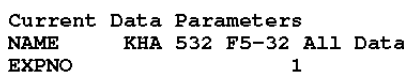

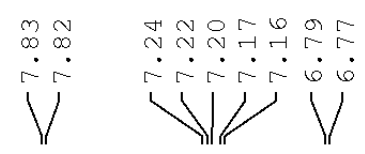

EXPNO

F2 - Acquisition Parameters

Date__ 20200702

Time

spect
PRSTRUD $5 \mathrm{~mm}$ PABBO BB/

PULPROG

TD

NS

$\begin{array}{ll} & 9009.009 \mathrm{~Hz} \\ \text { FWH } & 0.274933 \mathrm{HRES}\end{array}$

$\begin{array}{ll}\text { FID } & 0.274933 \mathrm{~Hz} \\ \text { RG } & 1.8186240 \mathrm{sec}\end{array}$

$\begin{array}{lr}\text { RG } & 135.71 \\ \text { DW } & 55.500 \text { usec } \\ \text { DE } & 7.62 \text { usec }\end{array}$

$\begin{array}{lr}\mathrm{DE} & 298.0 \mathrm{~K} \\ \mathrm{TE} & 2.00000000 \mathrm{sec}\end{array}$

TDO

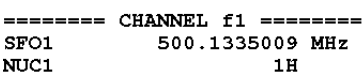

$\begin{array}{lr}\text { NUC1 } & 1 \mathrm{H} \\ \text { P1 } & 14.00 \text { usec } \\ \text { PLW1 } & 19.95299911 \mathrm{~W}\end{array}$

F2 - Processing parameter

SI $\quad 32768$

SF $\quad 500.1300219 \mathrm{MHz}$

$\begin{array}{ll}\text { SSB } & 0 \\ \mathrm{LB} & 0 \mathrm{~Hz}\end{array}$

$\begin{array}{lll}\mathrm{GB} & 0 & 1.00\end{array}$

1.00

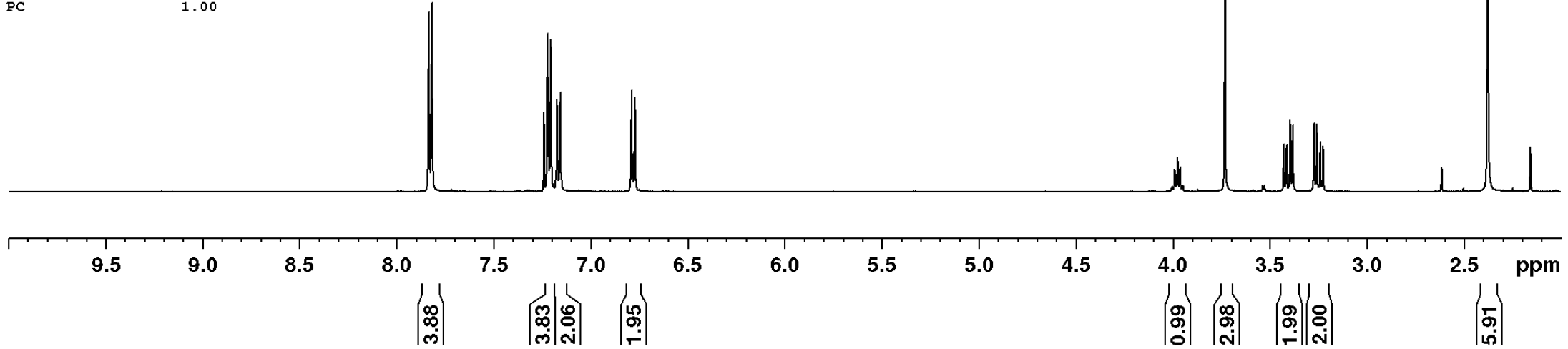

Figure S50. ${ }^{1} \mathrm{H}$ NMR spectrum of compound $4 c\left(500 \mathrm{MHz}, \mathrm{CDCl}_{3}, 25{ }^{\circ} \mathrm{C}\right)$ 


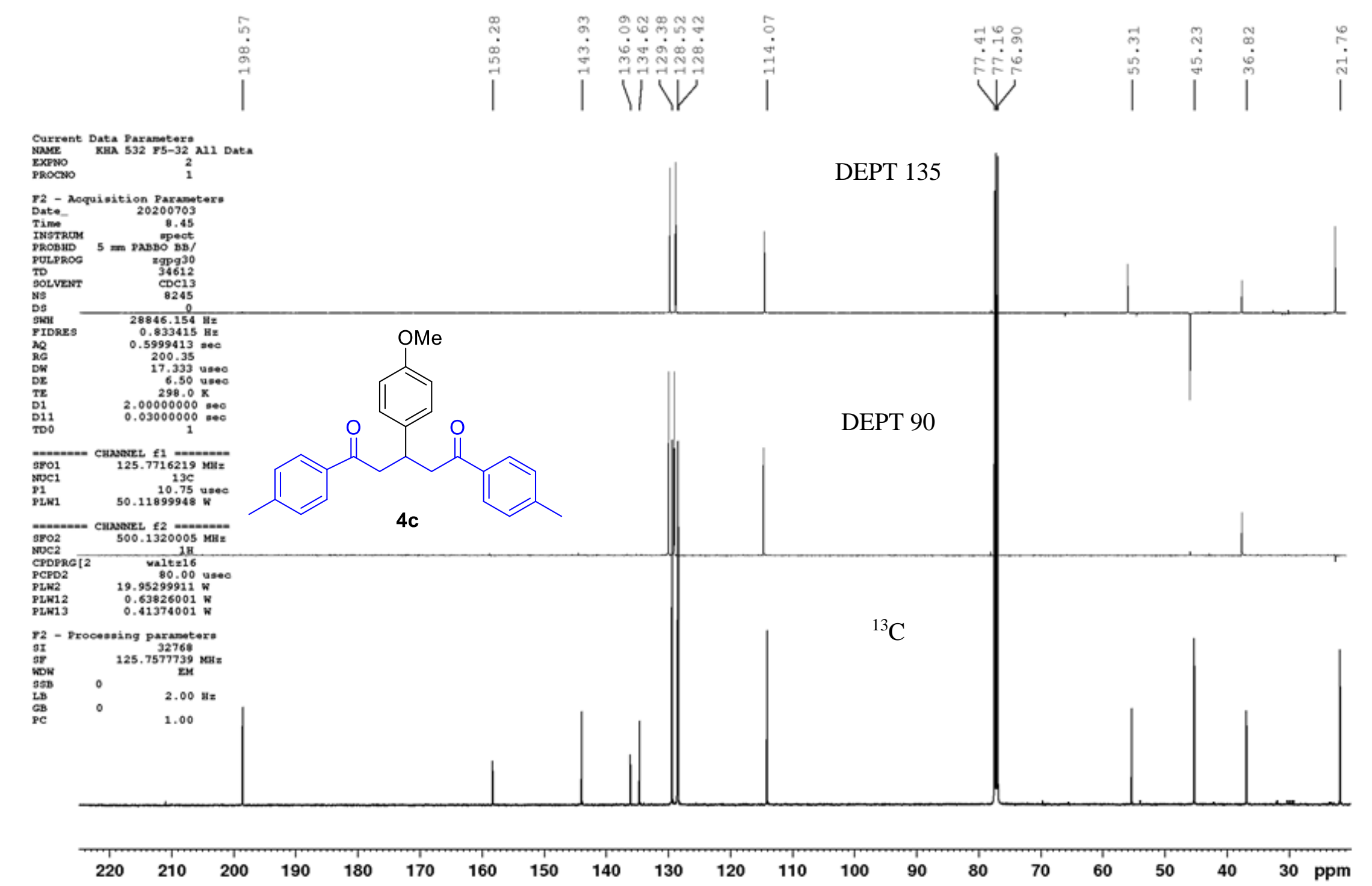

Figure S51. ${ }^{13} \mathrm{C}$ NMR spectrum of compound $4 \mathbf{c}\left(125 \mathrm{MHz}, \mathrm{CDCl}_{3}, 25{ }^{\circ} \mathrm{C}\right)$ 
Current Data Parameters
NAME KHA 531 F52-86 All Data

$$
\begin{aligned}
& \text { NAME KHA } 531 \text { F52-86 All Data } \\
& \text { PROCNO } \\
& \text { F2 - Acquisition Parameters } \\
& \begin{array}{lr}
\text { Date } & 20200701 \\
\text { Time } & 21.58
\end{array} \\
& \begin{array}{lr}
\text { INSTRUM } & \text { spect } \\
\text { PROBHD } & 5 \mathrm{~mm} \text { CPPBO BB } \\
\text { PULPROG } & \mathrm{zg} 30
\end{array} \\
& \begin{array}{lr}
\text { TD } & \text { zg30 } \\
\text { SOLVENT } & 32768 \\
\text { CDSC13 }
\end{array} \\
& \begin{array}{lc}
\text { NS } & 8 \\
\text { DS } & 0 \\
\text { SWH } & 8992.806 \mathrm{~Hz}
\end{array} \\
& \begin{array}{lr}
\text { SWH } & 8992.806 \mathrm{~Hz} \\
\text { FIDRES } & 0.274439 \mathrm{~Hz} \\
\text { AO } & 1.8219008 \mathrm{sec}
\end{array} \\
& \begin{array}{lc}
\text { AQ } & 1.8219008 \mathrm{sec} \\
\text { RG } & 90.5
\end{array} \\
& \begin{array}{lr}
\mathrm{DW} & 55.600 \text { usec } \\
\mathrm{DE} & 25.00 \mathrm{usec}
\end{array} \\
& \begin{array}{lr}
\mathrm{TE} & 298.0 \mathrm{~K} \\
\mathrm{D} 1 & 2.00000000 \mathrm{sec}
\end{array}
\end{aligned}
$$

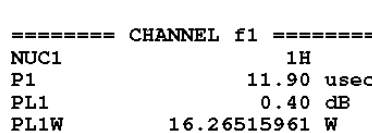

$$
\begin{aligned}
& \begin{array}{lr}
\text { PL1 } & 0.40 \mathrm{~dB} \\
\text { PL1W } & 16.26515961 \mathrm{~W} \\
\text { SFO1 } & 500.2035014 \mathrm{MHz}
\end{array} \\
& \text { F2 - Processing parameters } \\
& \begin{array}{lr}
\text { SI } & 32768 \\
\text { SF } & 500.2000210 \mathrm{MHz}
\end{array} \\
& \text { WDW } \\
& \begin{array}{lll}
\mathrm{SSB} & 0 \\
\mathrm{LB} & 0 \mathrm{~Hz} \\
\mathrm{~GB} & 0
\end{array} \\
& \text { PC } 1.00
\end{aligned}
$$
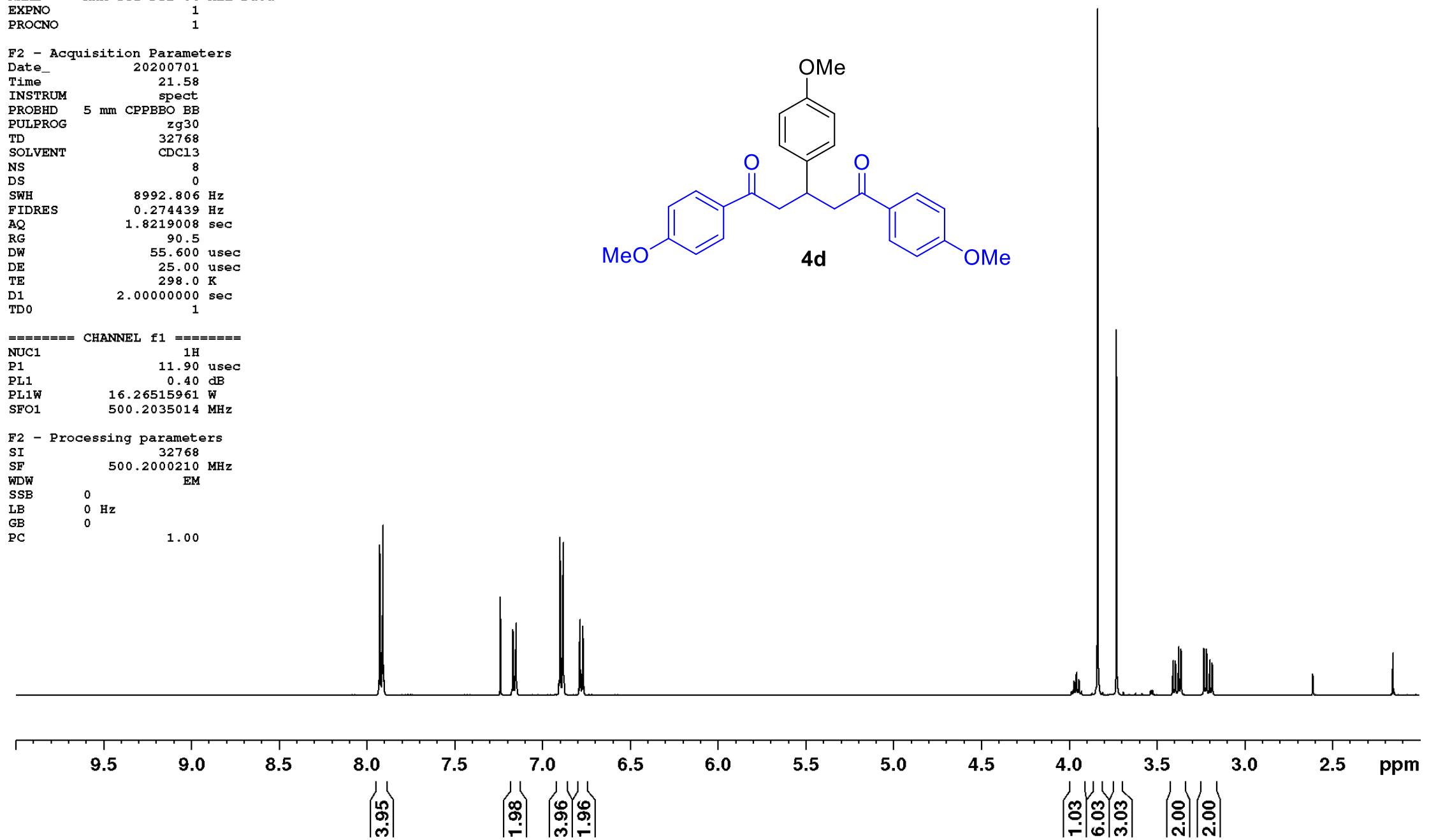

Figure S52. ${ }^{1} \mathrm{H}$ NMR spectrum of compound $4 \mathbf{d}\left(500 \mathrm{MHz}, \mathrm{CDCl}_{3}, 25{ }^{\circ} \mathrm{C}\right)$ 


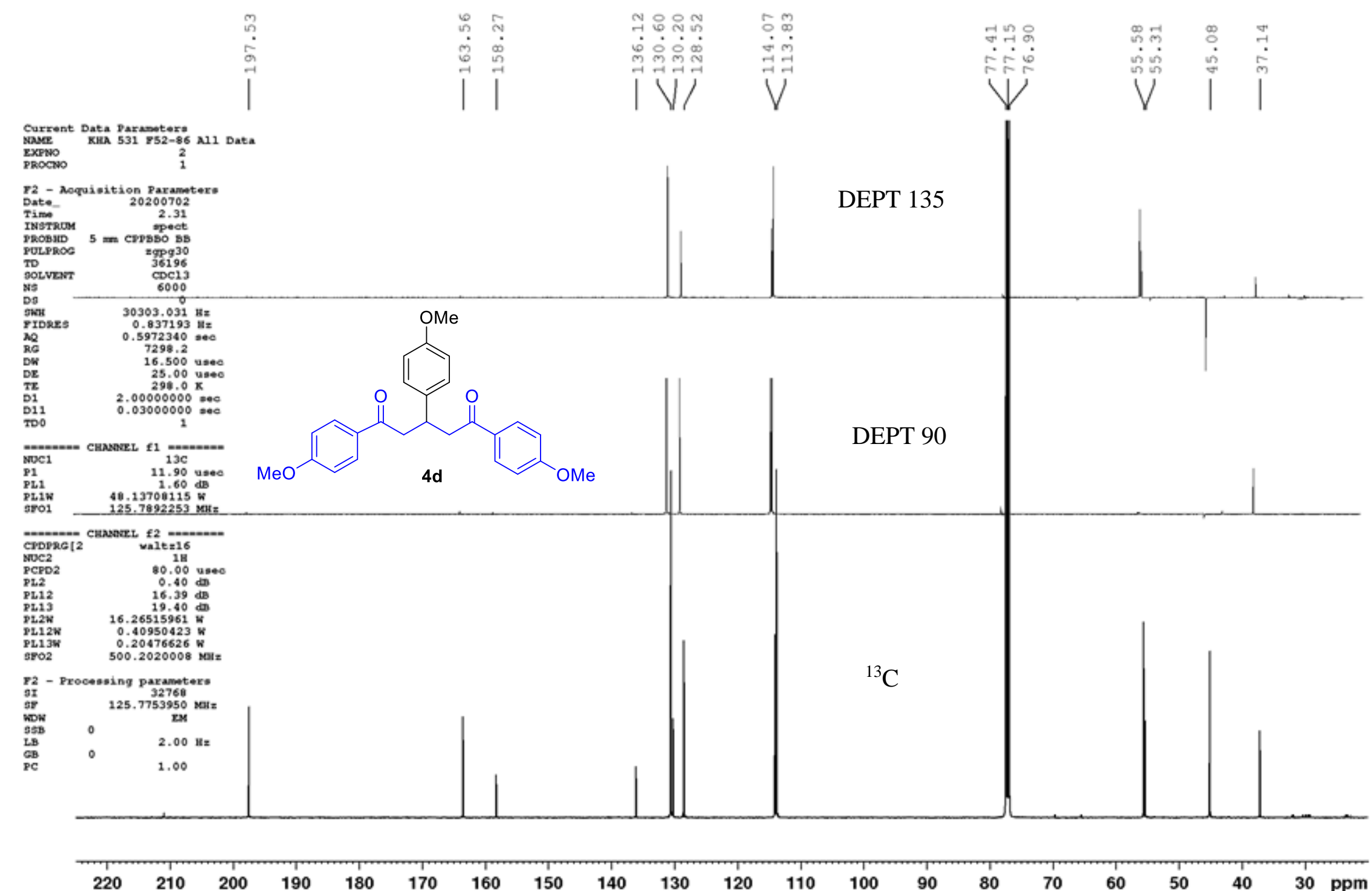

Figure S53. ${ }^{13} \mathrm{C}$ NMR spectrum of compound $4 d\left(125 \mathrm{MHz}, \mathrm{CDCl}_{3}, 25{ }^{\circ} \mathrm{C}\right)$ 
Current Data Parameters
NAME
KHA 537 F8-43 All Data

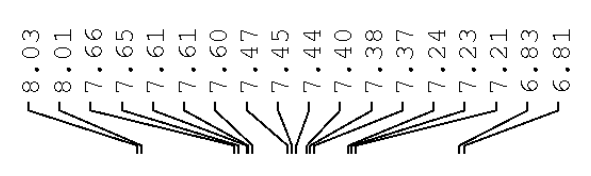

EXPNO
PROCNO

F2 - Acquisition Parameters
Date-20200711

$\begin{array}{lr}20200711 \\ \text { Time } & 22.07\end{array}$

INSTRUM
PROBHD $5 \mathrm{~mm}$ CPPBBO BB

PULPROG

$\begin{array}{ll}\text { TD } & 32768 \\ \text { SOLVENT } & \mathrm{CDC13}\end{array}$

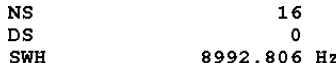

$\begin{array}{ll}\text { SWH } & 8992.806 \mathrm{~Hz} \\ \text { FIDRES } & 0.274439 \mathrm{~Hz}\end{array}$

AQ $\quad 1.8219008 \mathrm{sec}$

90.5
55.600 usec

55.600 usec
25.00 usec

$\begin{array}{lr}\text { TE } & 298.1 \mathrm{~K} \\ \text { D1 } & 2.00000000 \mathrm{sec}\end{array}$

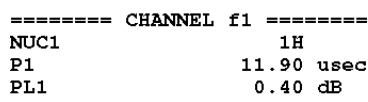

$\begin{array}{lr}\text { PL1 } & 0.40 \mathrm{~dB} \\ \text { PL1W } & 16.26515961 \mathrm{~W}\end{array}$

F2 - Processing parameters

SI $\quad 32768$

WDW $\quad 500.2000210 \mathrm{MHz}$

$\begin{array}{ll}\text { SSB } & 0 \\ \text { LB } & 0 \\ \text { GB } & 0\end{array}$

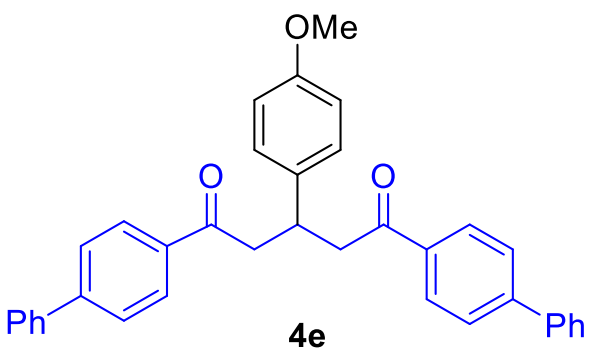

$\begin{array}{lll}\mathrm{GB} & 0 & 1.00\end{array}$

(1)
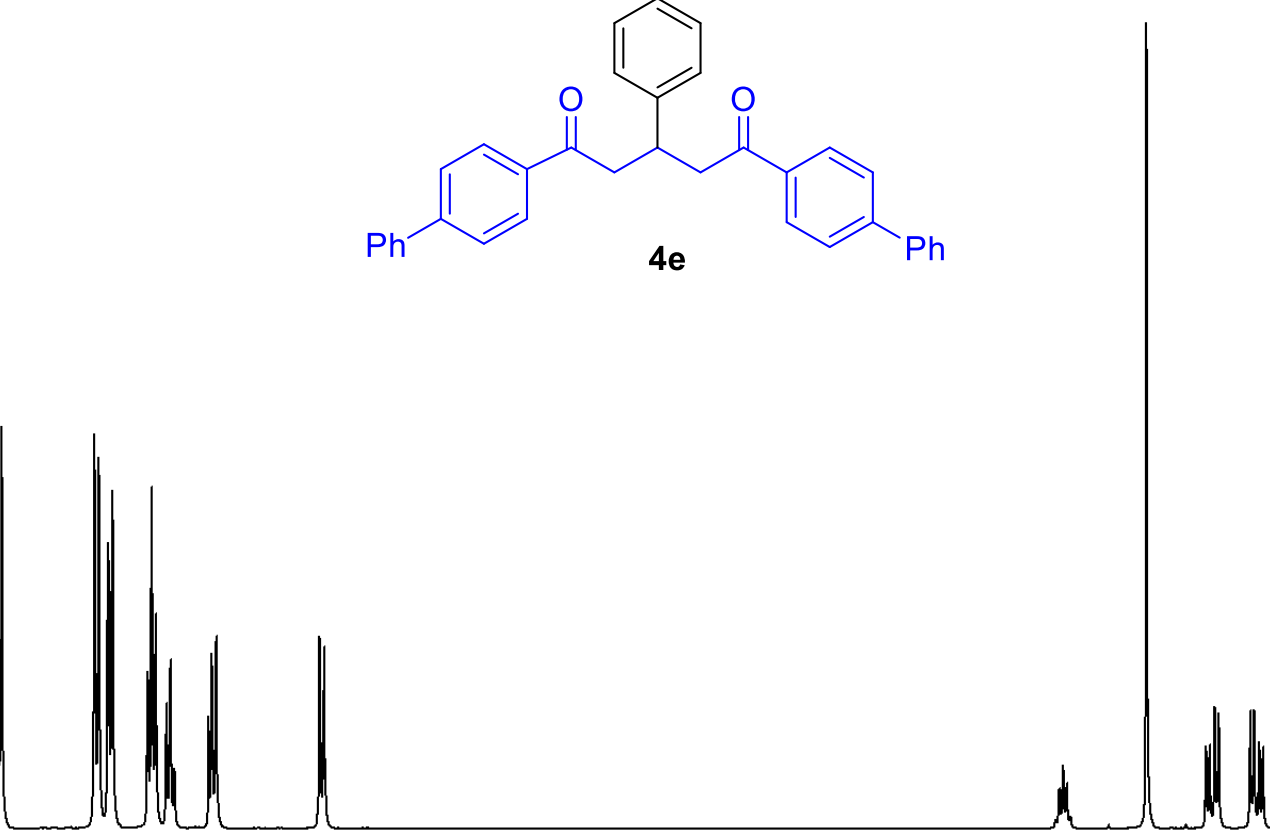

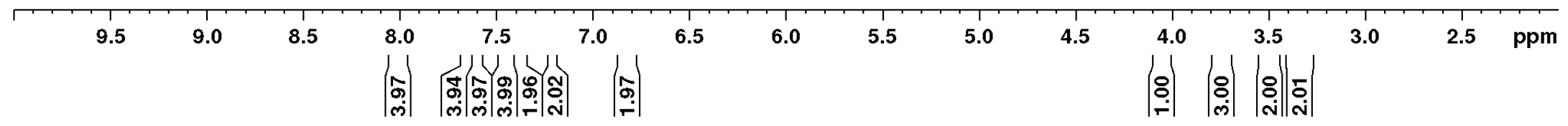

Figure S54. ${ }^{1} \mathrm{H}$ NMR spectrum of compound $4 \mathrm{e}\left(500 \mathrm{MHz}, \mathrm{CDCl}_{3}, 25{ }^{\circ} \mathrm{C}\right)$ 
Current Data Parameters
NAME
KHA 537 F8-43 All Data
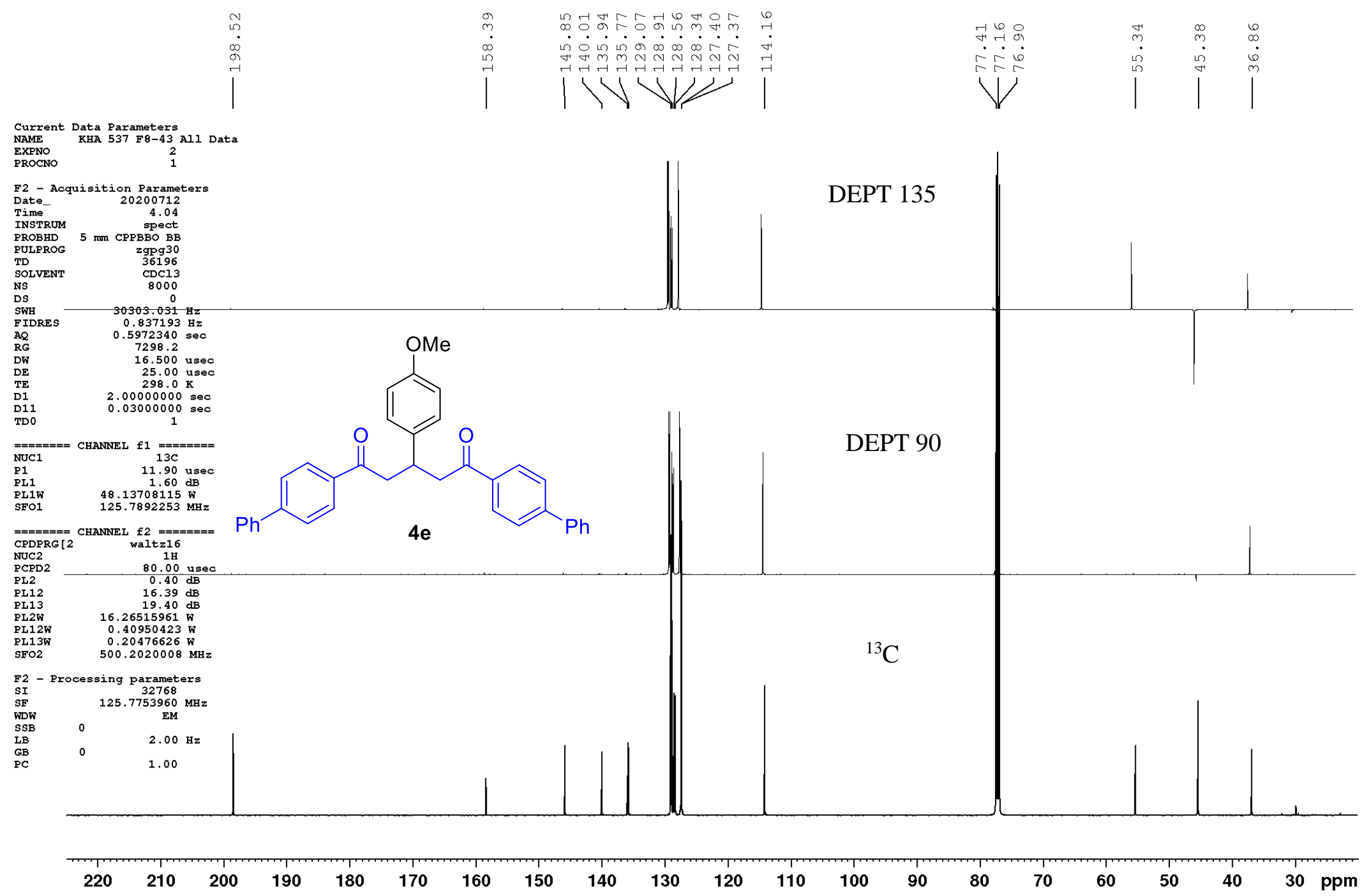

Figure S55. ${ }^{13} \mathrm{C}$ NMR spectrum of compound $4 \mathrm{e}\left(125 \mathrm{MHz}, \mathrm{CDCl}_{3}, 25{ }^{\circ} \mathrm{C}\right)$ 


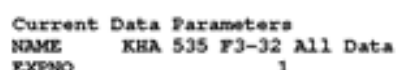

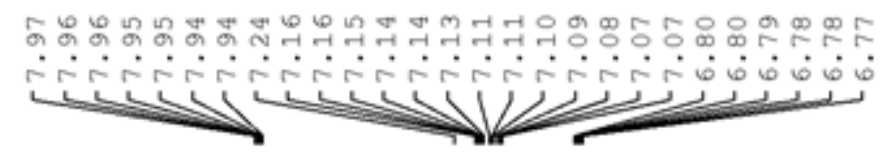

PROCONO

P2 - Aoquisition Parameters

$\begin{array}{rr}\text { Date- } & 20200709 \\ \text { Timo } & 22.19\end{array}$

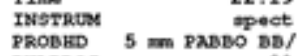

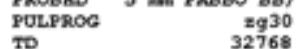

$\begin{array}{ll}\text { TD } & 32768 \\ \text { gotVEN: } & \text { CDC13 }\end{array}$

$\begin{array}{ll}\mathrm{Ns} & 16 \\ \mathrm{DS} & 0\end{array}$

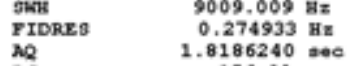

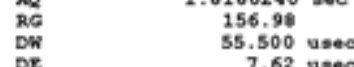

$\begin{array}{ll}\mathrm{DE} & 7.62 \text { usec } \\ \mathrm{TE} & 298.0 \mathrm{~K}\end{array}$

D1 $\quad 2.00000000$ ate

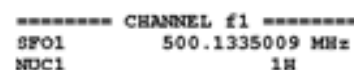

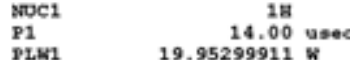

F2 - Processing parameter

II $500.1300221 \mathrm{MB}$

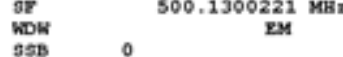

$\begin{array}{lll}\mathrm{gSB} & 0 & \\ \mathrm{LB} & \vdots \mathrm{Hz} & \\ \mathrm{GB} & \vdots & 1.00\end{array}$
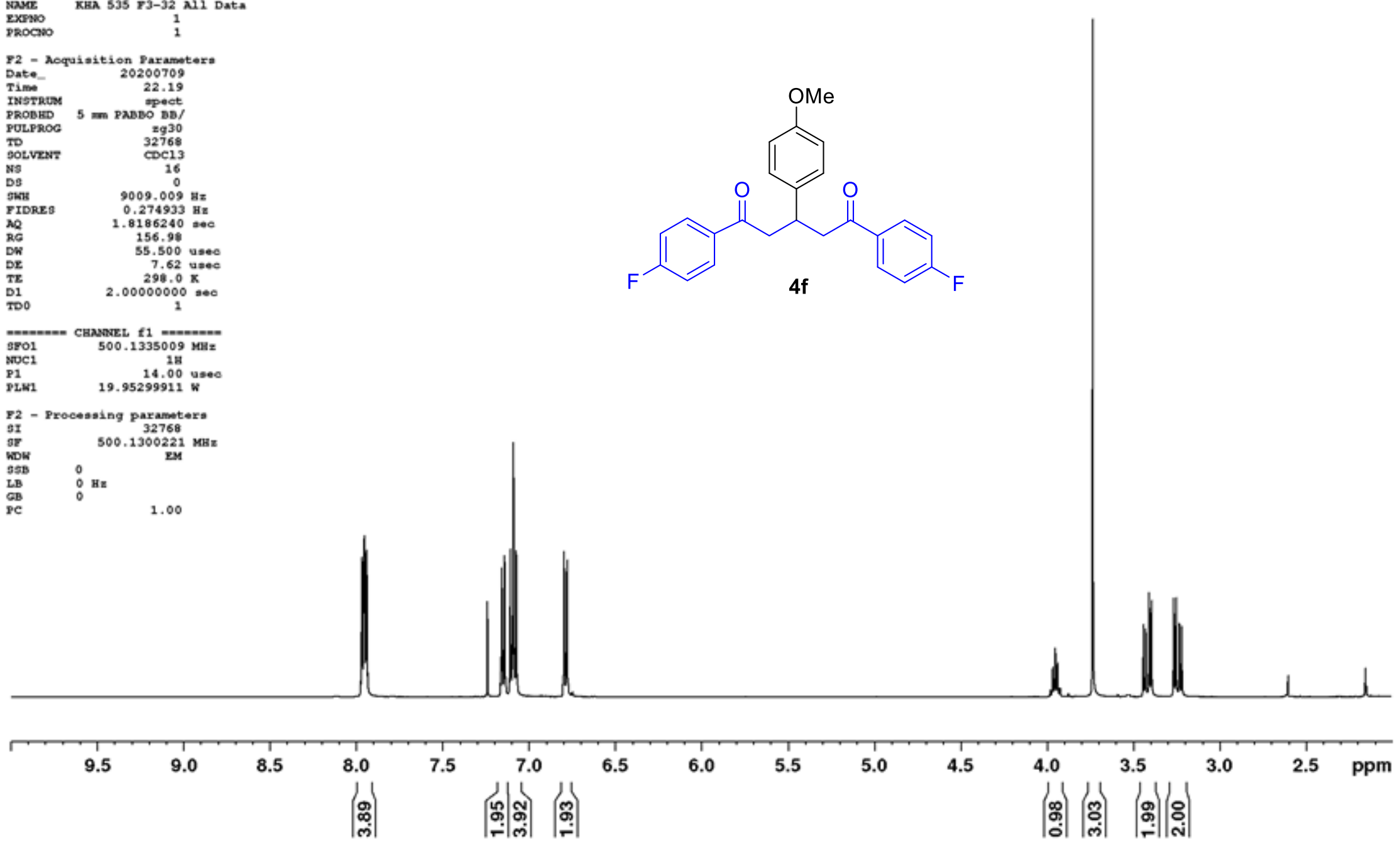

Figure S56. ${ }^{1} \mathrm{H}$ NMR spectrum of compound $4 f\left(500 \mathrm{MHz}, \mathrm{CDCl}_{3}, 25{ }^{\circ} \mathrm{C}\right)$ 


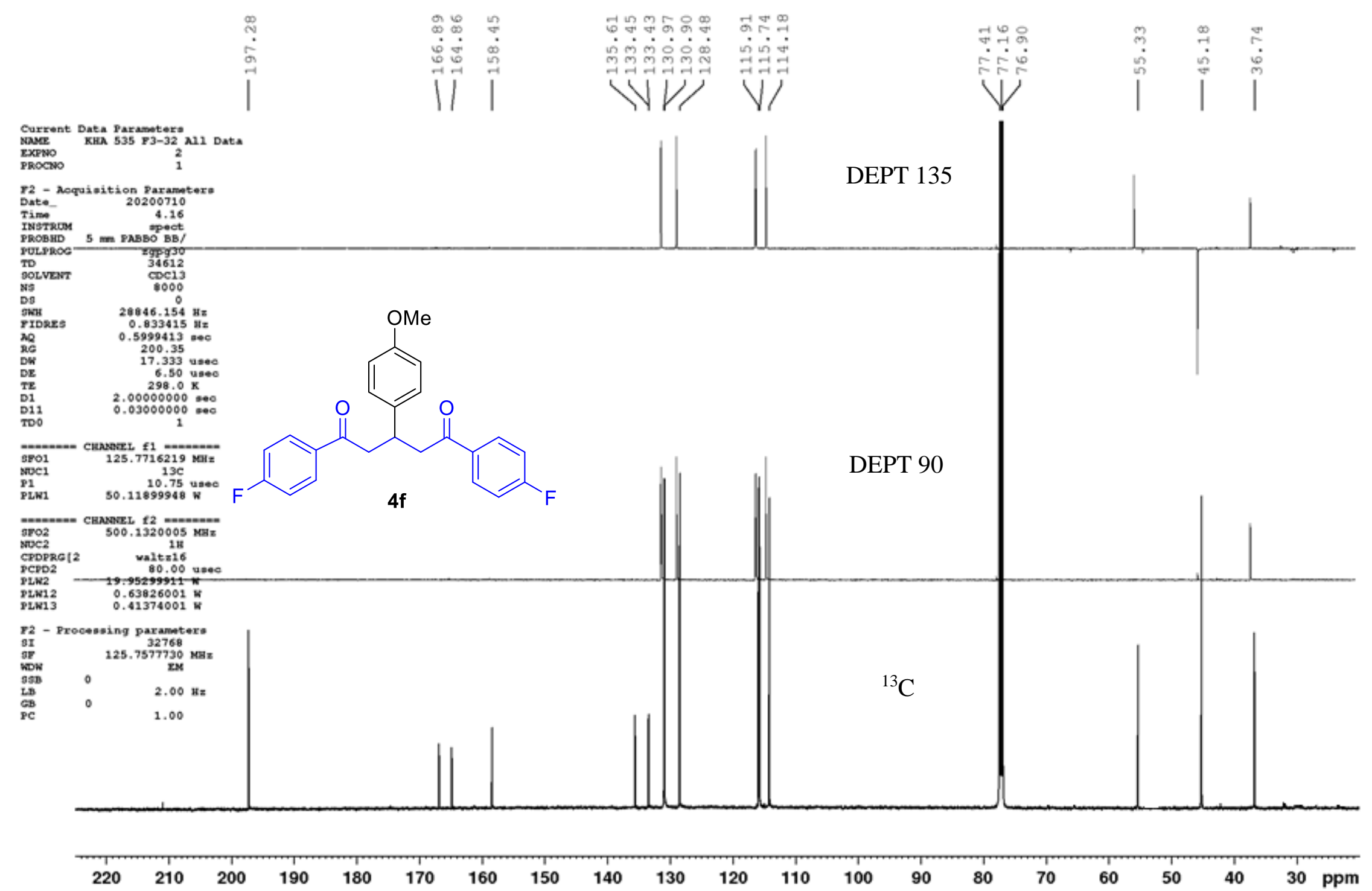

Figure S57. ${ }^{13} \mathrm{C}$ NMR spectrum of compound $\mathbf{4 f}\left(125 \mathrm{MHz}, \mathrm{CDCl}_{3}, 25{ }^{\circ} \mathrm{C}\right)$ 
Current Data Parameters
NAME KHA 536 F3-20 All Data

EXPNO

1
1

F2 - Acquisition Parameter

Time

PROBHD $5 \mathrm{~mm}$ PABBO BB/
PULPROG

\begin{tabular}{lr}
$\mathrm{zg} 30$ \\
TD & 32768 \\
\hline
\end{tabular}

$\begin{array}{lc}\text { DS } & 0 \\ \text { SWH } & 9009.009 \mathrm{~Hz} \\ \text { FIDRES } & 0.274933 \mathrm{~Hz}\end{array}$

$\begin{array}{lr}\text { AQ } & 0.274933 \mathrm{~Hz} \\ \text { RQ } & 1.8186240 \mathrm{sec}\end{array}$

$\begin{array}{ll}\mathrm{RG} & 135.71 \\ \mathrm{DW} & 55.500 \mathrm{usec}\end{array}$

$\begin{array}{lr}\mathrm{DE} & 7.62 \mathrm{usec} \\ \mathrm{TE} & 299.3 \mathrm{~K} \\ \mathrm{D} 1 & 2.00000000 \mathrm{sec}\end{array}$

$\begin{array}{ll}======= & \text { CHANNEL } f 11======== \\ \text { SFO1 } & 500.1335009 \mathrm{MHz}\end{array}$

$\begin{array}{lr}\text { NUC1 } & 1 \mathrm{H} \\ \mathrm{P} 1 & 14.00 \text { use }\end{array}$

F2 - Processing parameters

SI $\quad 32768$

$\begin{array}{llr}\text { SF } & & 500.1300219 \\ \text { WDW } & & \text { MHz } \\ \text { SSB } & 0 & \\ \text { LB } & 0 & \\ \text { GB } & 0 & \end{array}$

$\begin{array}{lll}\mathrm{GB} & 0 & 1.00 \\ \mathrm{PC} & \end{array}$

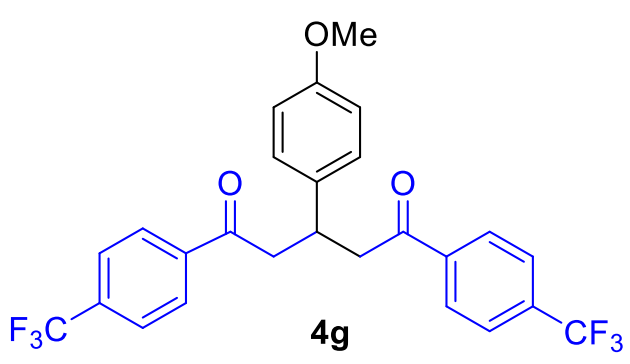

.00
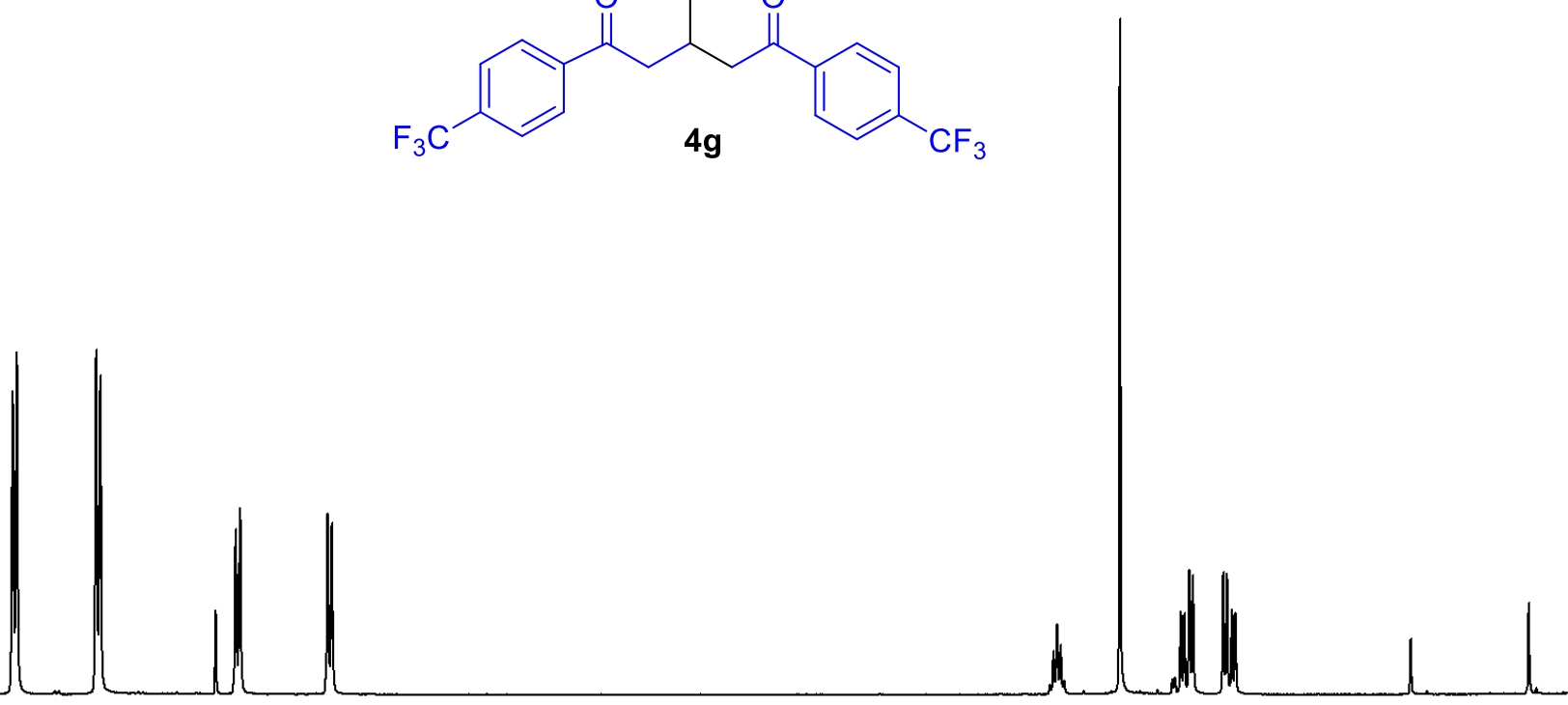

9.

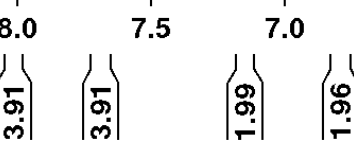
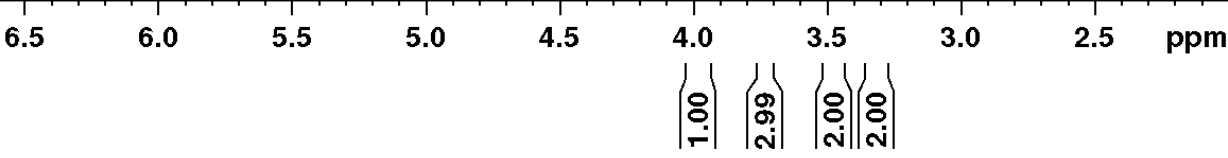

Figure S58. ${ }^{1} \mathrm{H}$ NMR spectrum of compound $4 \mathrm{~g}\left(500 \mathrm{MHz}, \mathrm{CDCl}_{3}, 25{ }^{\circ} \mathrm{C}\right)$ 


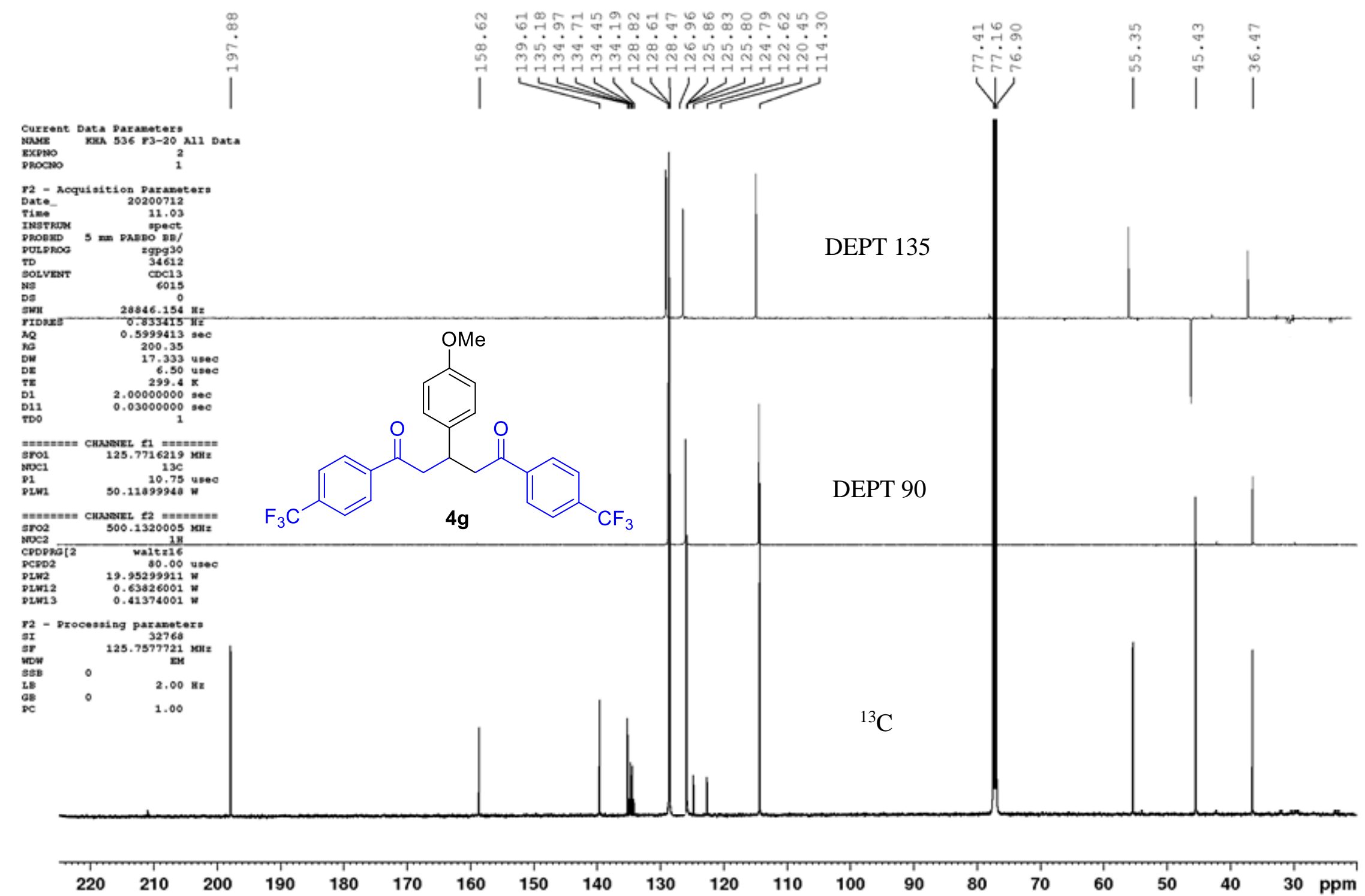

Figure S59. ${ }^{13} \mathrm{C}$ NMR spectrum of compound $\mathbf{4 g}\left(125 \mathrm{MHz}, \mathrm{CDCl}_{3}, 25{ }^{\circ} \mathrm{C}\right)$ 
Current Data Parameters
NaNG
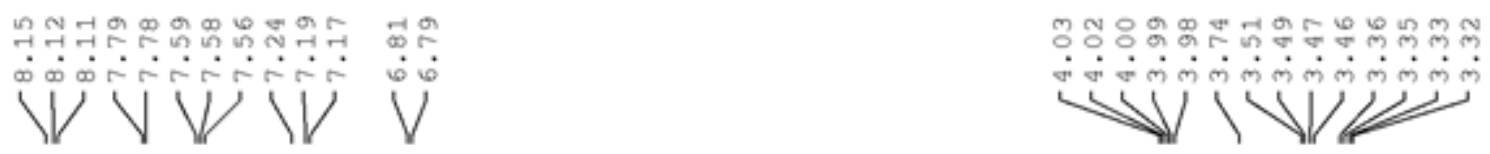

\section{EXPNO}

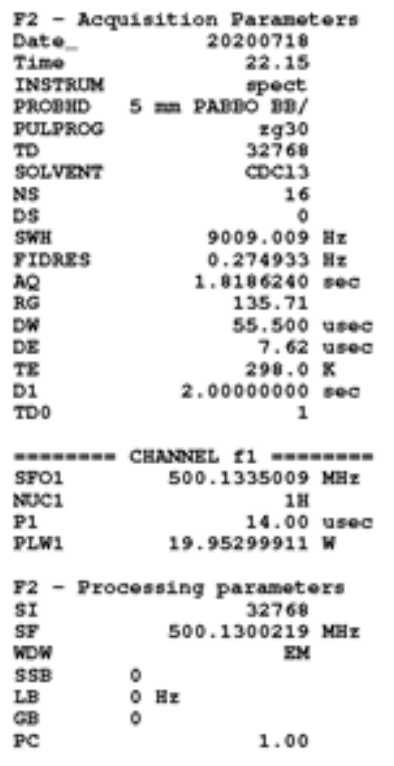
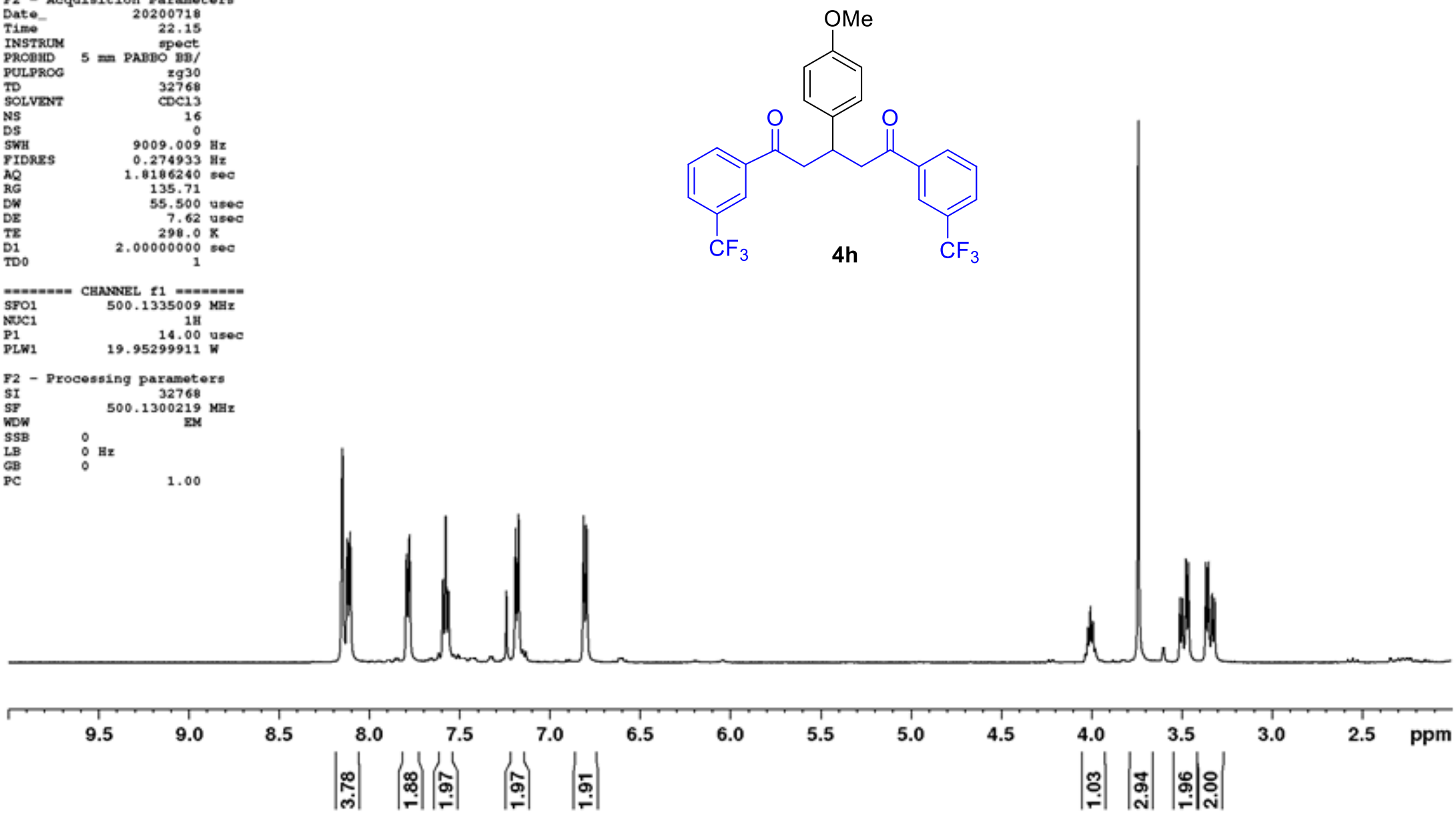

Figure S60. ${ }^{1} \mathrm{H}$ NMR spectrum of compound $4 \mathbf{h}\left(500 \mathrm{MHz}, \mathrm{CDCl}_{3}, 25{ }^{\circ} \mathrm{C}\right)$ 


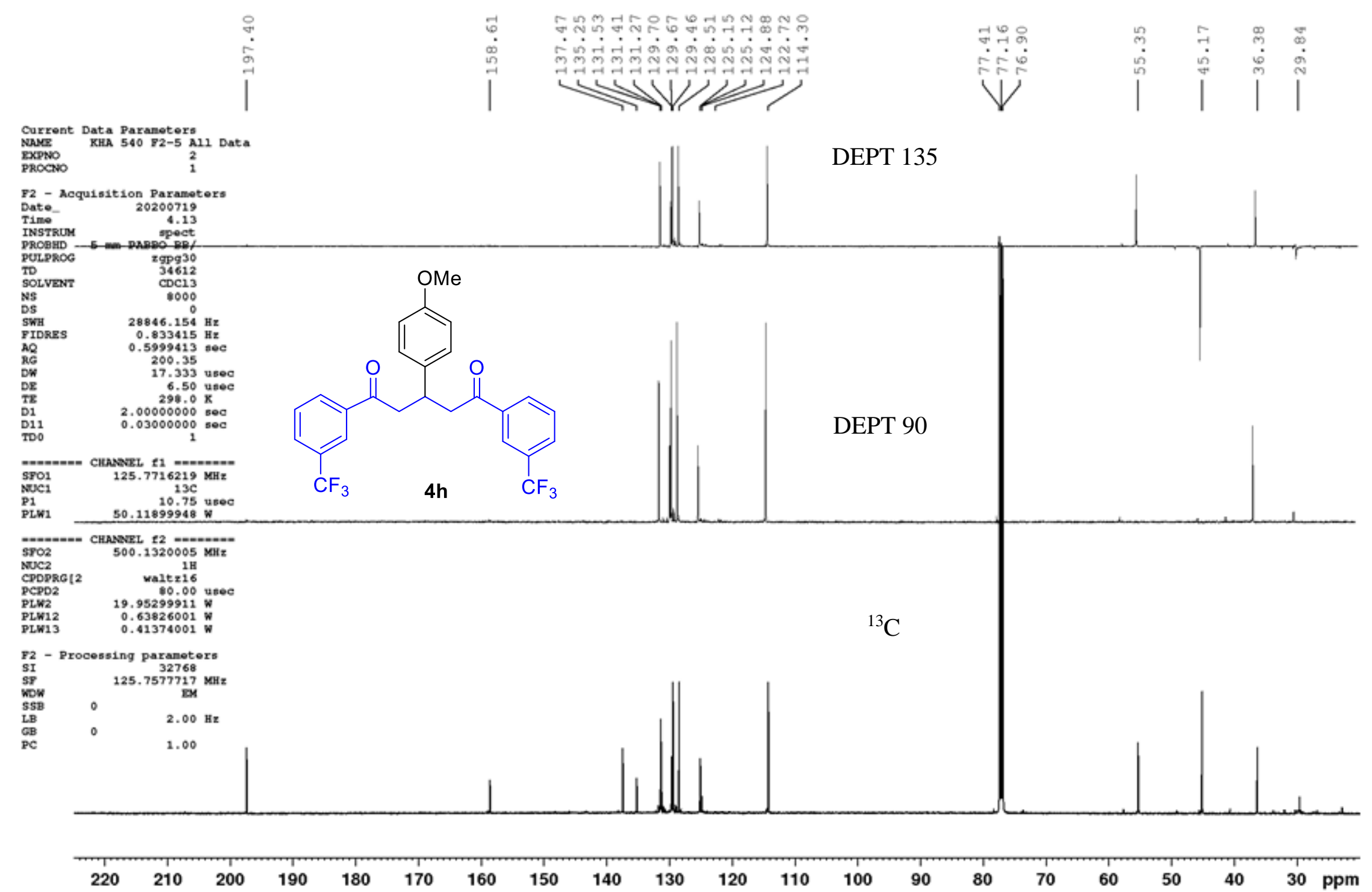

Figure S61. ${ }^{13} \mathrm{C}$ NMR spectrum of compound $4 \mathbf{h}\left(125 \mathrm{MHz}, \mathrm{CDCl}_{3}, 25{ }^{\circ} \mathrm{C}\right)$ 
Current Data Parameters
NAME
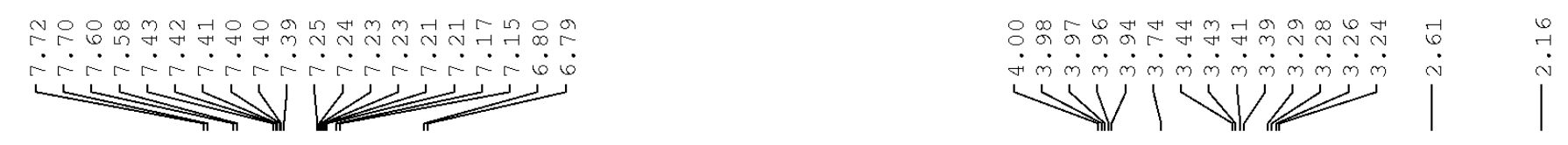

\section{EXPNO}
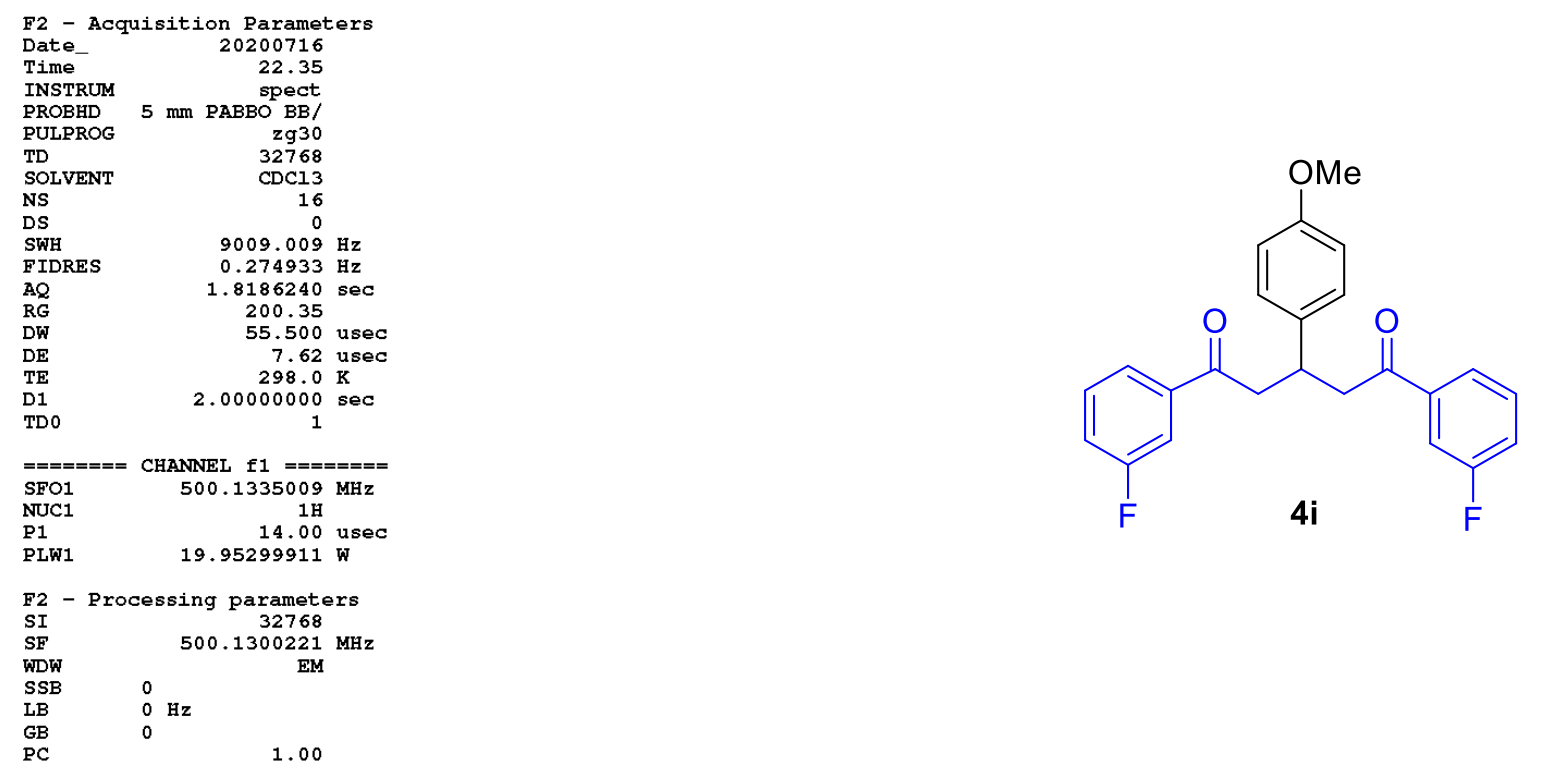

F2 - Processing parameters

$\begin{array}{llc}\text { SI } & & 32768 \\ \text { SF } & & 500.1300221 \\ \text { WDW } & & \text { MHz } \\ \text { SSB } & 0 & \\ \text { LB } & 0 & \text { Hz } \\ \text { GB } & 0 & \end{array}$
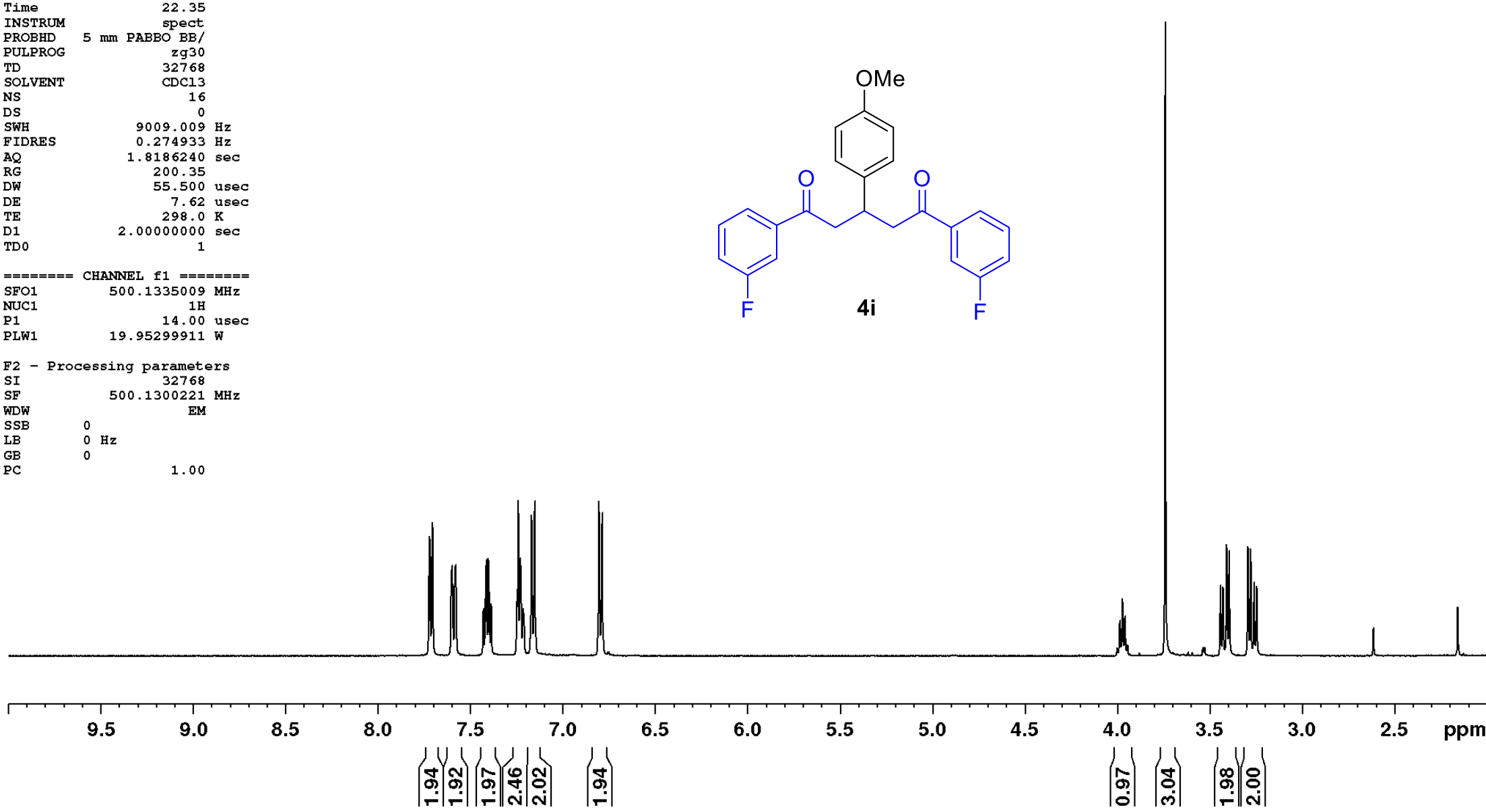

Figure S62. ${ }^{1} \mathrm{H}$ NMR spectrum of compound $4 \mathbf{i}\left(500 \mathrm{MHz}, \mathrm{CDCl}_{3}, 25{ }^{\circ} \mathrm{C}\right)$ 

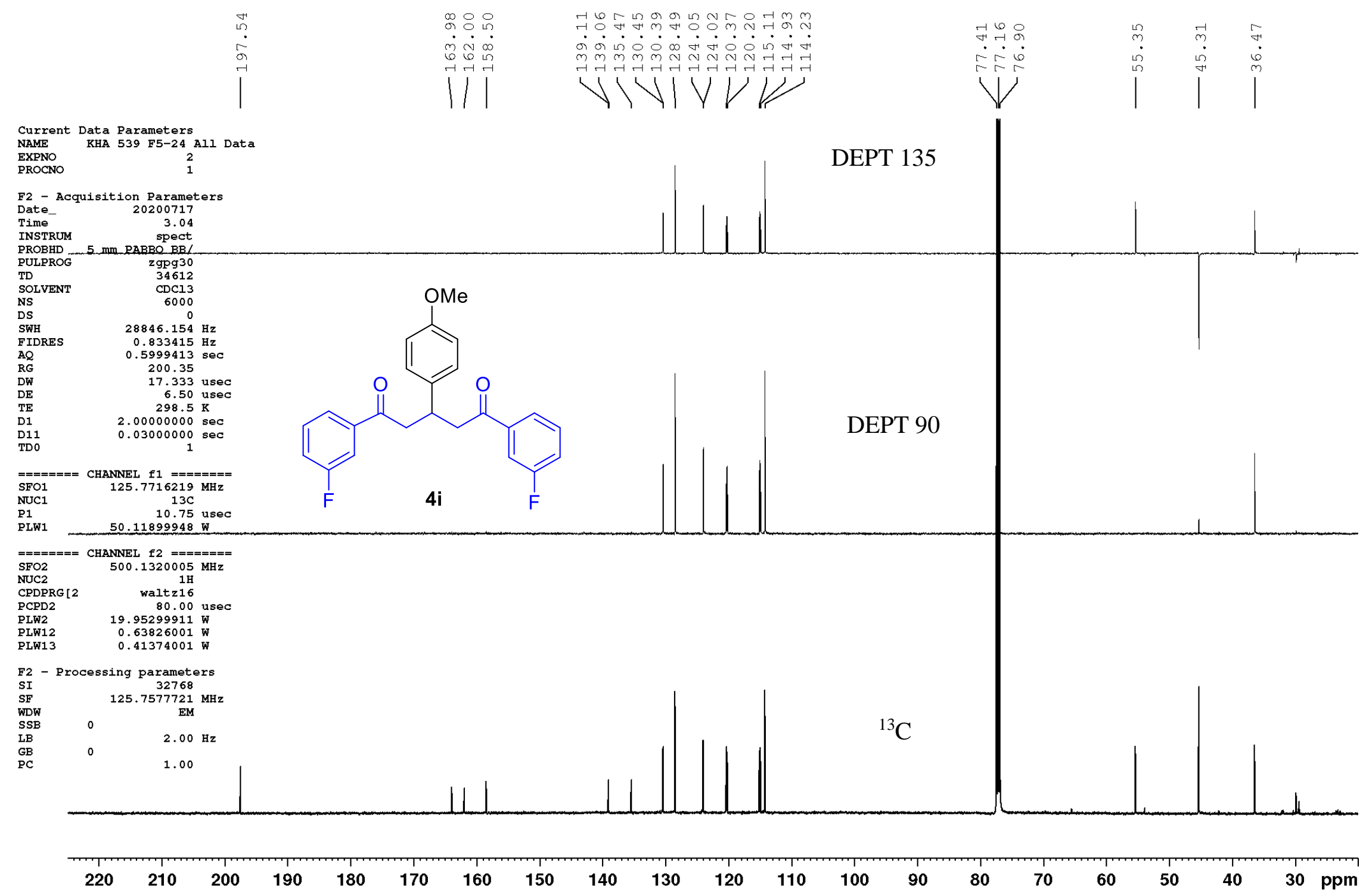

Figure S63. ${ }^{13} \mathrm{C}$ NMR spectrum of compound $4 \mathbf{i}\left(125 \mathrm{MHz}, \mathrm{CDCl}_{3}, 25{ }^{\circ} \mathrm{C}\right)$ 
Current Data Paranetera

Exase
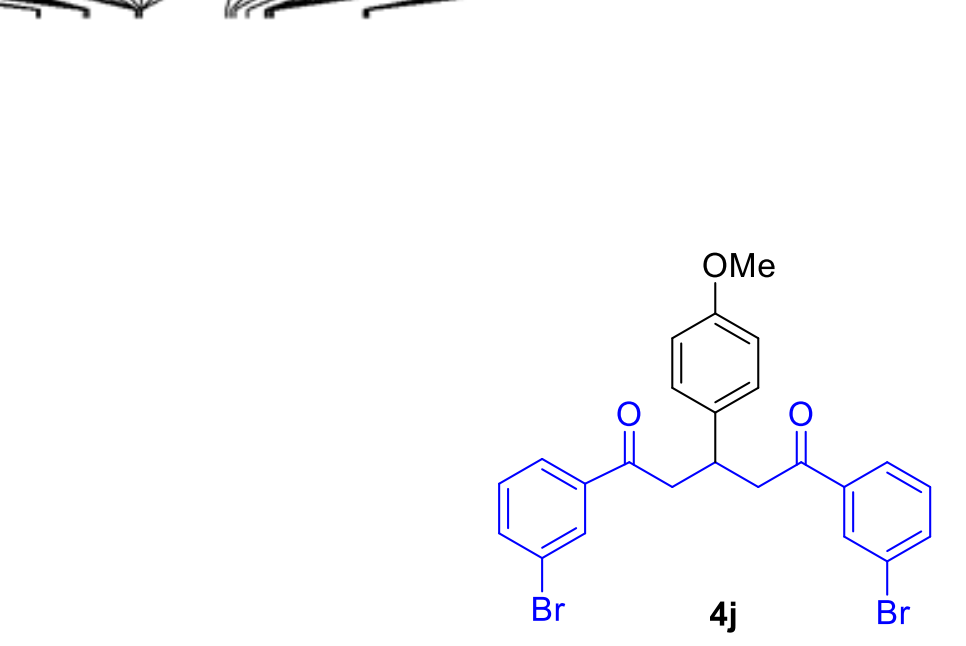

Y2 - Mequisition Parameters
20200714

Timo

Inestrom 5

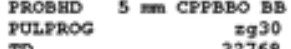

TD

$\begin{array}{lc}\text { NS } & 16 \\ \text { Dg } & 0 \\ \text { gWB } & 8992.806\end{array}$

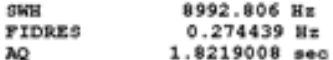

$\begin{array}{lr}\text { MQ } & 1.8219008=0 \\ R G & 90.5 \\ D W & 55.600 \text { usec }\end{array}$

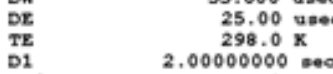

TDO

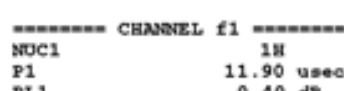

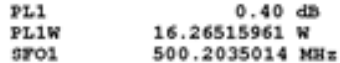

P2 - Processing parameters

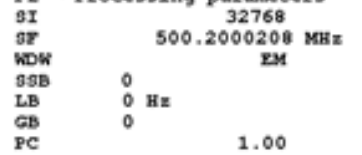

4j
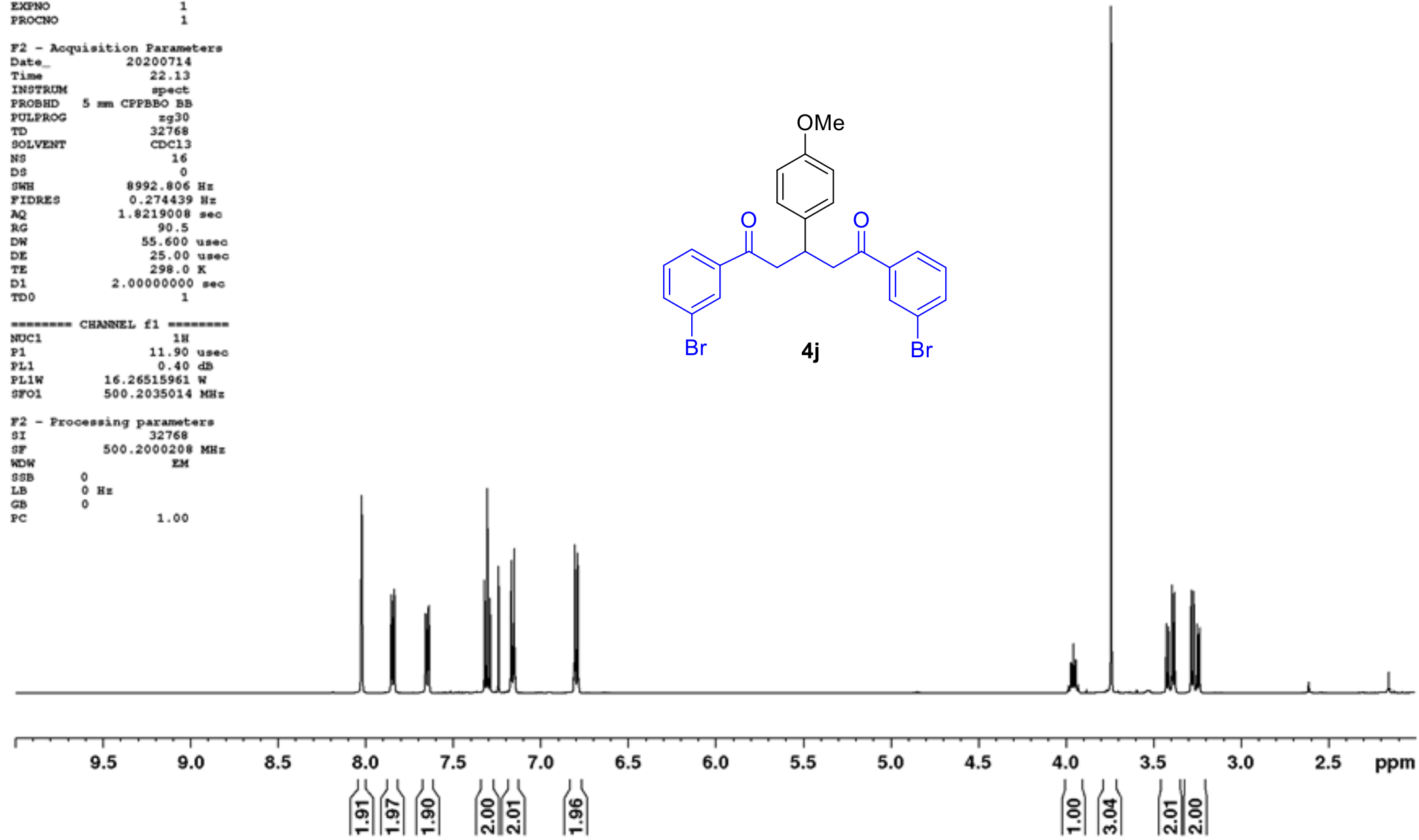

Figure S64. ${ }^{1} \mathrm{H}$ NMR spectrum of compound $4 \mathbf{j}\left(500 \mathrm{MHz}, \mathrm{CDCl}_{3}, 25{ }^{\circ} \mathrm{C}\right.$ ) 


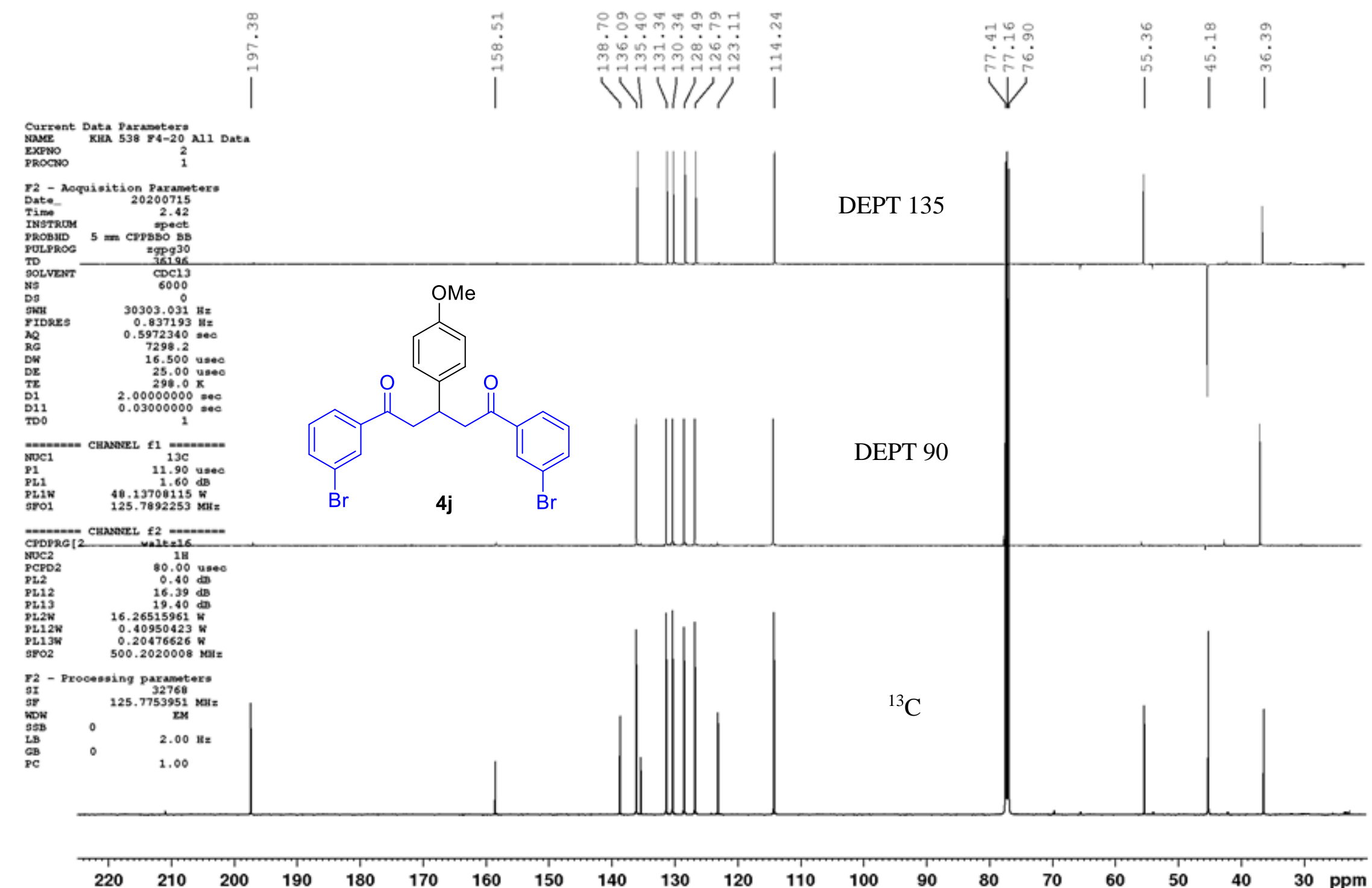

Figure S65. ${ }^{13} \mathrm{C}$ NMR spectrum of compound $\mathbf{4 j}\left(125 \mathrm{MHz}, \mathrm{CDCl}_{3}, 25{ }^{\circ} \mathrm{C}\right)$ 
Current Data Parameters

Data

EXPNO

F2 - Acquisition Parameter
Date_ 20200718

Time 22.31

PROBHD $5 \mathrm{~mm}$ CPPBBO BB

PULPROG

$\begin{array}{ll}\text { TD } & 32768 \\ \text { SOLVENT } & \mathrm{CDCl3}\end{array}$

NS 16

DS

$\begin{array}{ll}\text { SWH } & 8992.806 \mathrm{~Hz} \\ \text { FIDRES } & 0.274439 \mathrm{~Hz}\end{array}$

AQ $\quad 1.8219008 \mathrm{sec}$

RG $\quad 71.8$

$\begin{array}{lr}\mathrm{DW} & 55.600 \text { usec } \\ \mathrm{DE} & 25.00 \text { usec }\end{array}$

$\begin{array}{ll}\text { TE } & 2.00000000 \mathrm{sec} \\ \text { TD } & 1\end{array}$

$=======$ CHANNEL f1

NUC1

P1
PL1
PL1W

11.90 use
PL1W
$0.40 \mathrm{~dB}$

$\begin{array}{ll}16.26515961 \mathrm{~W} \\ \text { SFO1 } & 500.2035014 \mathrm{MHz}\end{array}$

F2 - Processing parameters

$\begin{array}{lc}\text { SI } & 32768 \\ \text { SF } & 500.2000209 \mathrm{MHz}\end{array}$

WDW $\quad 0 \quad$ EM

$\begin{array}{lll}\mathrm{LB} & 0 \mathrm{~Hz} \\ \mathrm{~GB} & 0\end{array}$
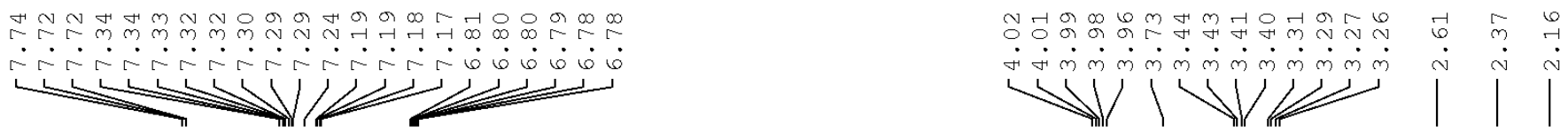

1.00
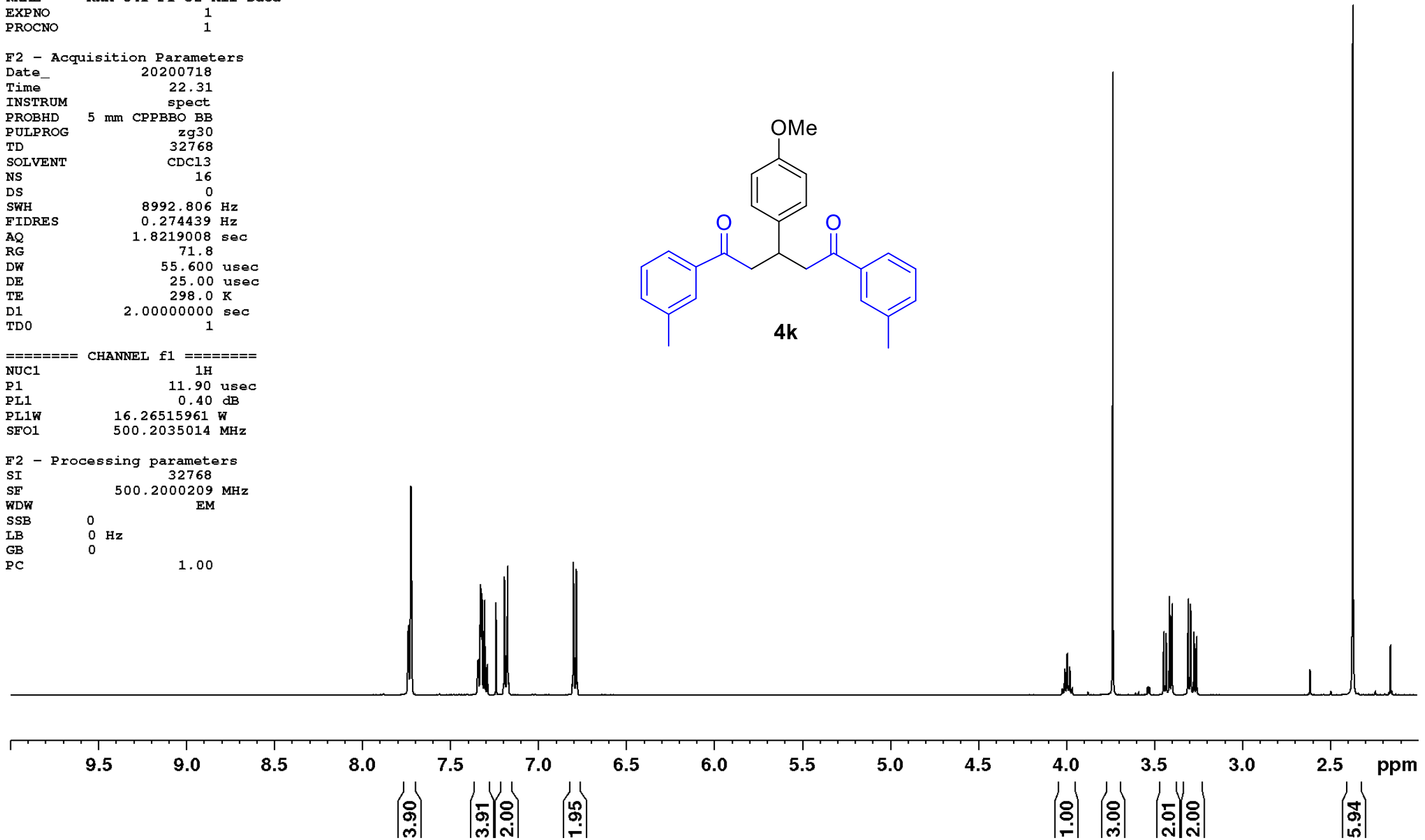

Figure S66. ${ }^{1} \mathrm{H}$ NMR spectrum of compound 4k $\left(500 \mathrm{MHz}, \mathrm{CDCl}_{3}, 25{ }^{\circ} \mathrm{C}\right)$ 


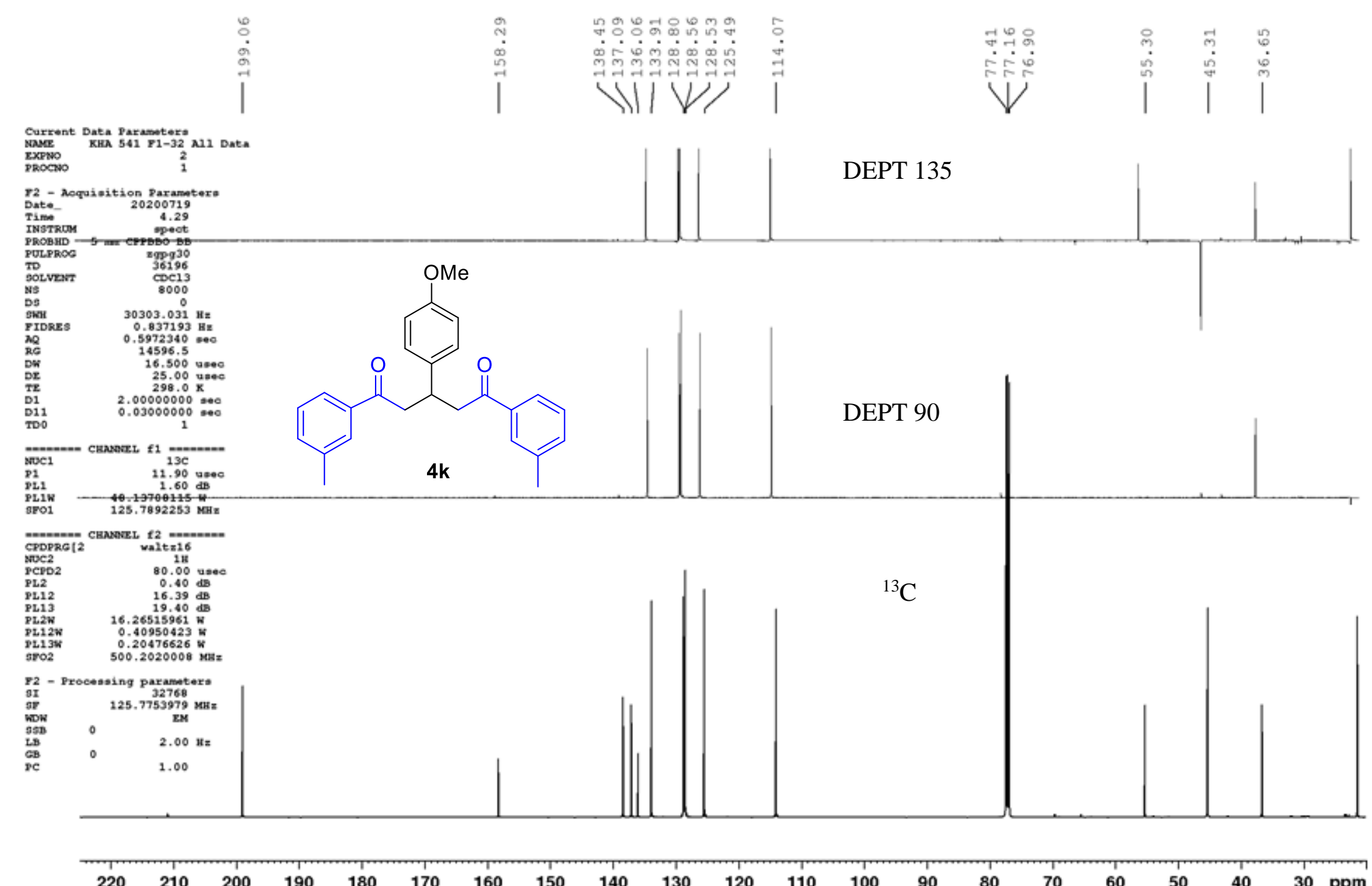

Figure S67. ${ }^{13} \mathrm{C}$ NMR spectrum of compound $4 \mathbf{k}\left(125 \mathrm{MHz}, \mathrm{CDCl}_{3}, 25{ }^{\circ} \mathrm{C}\right)$ 


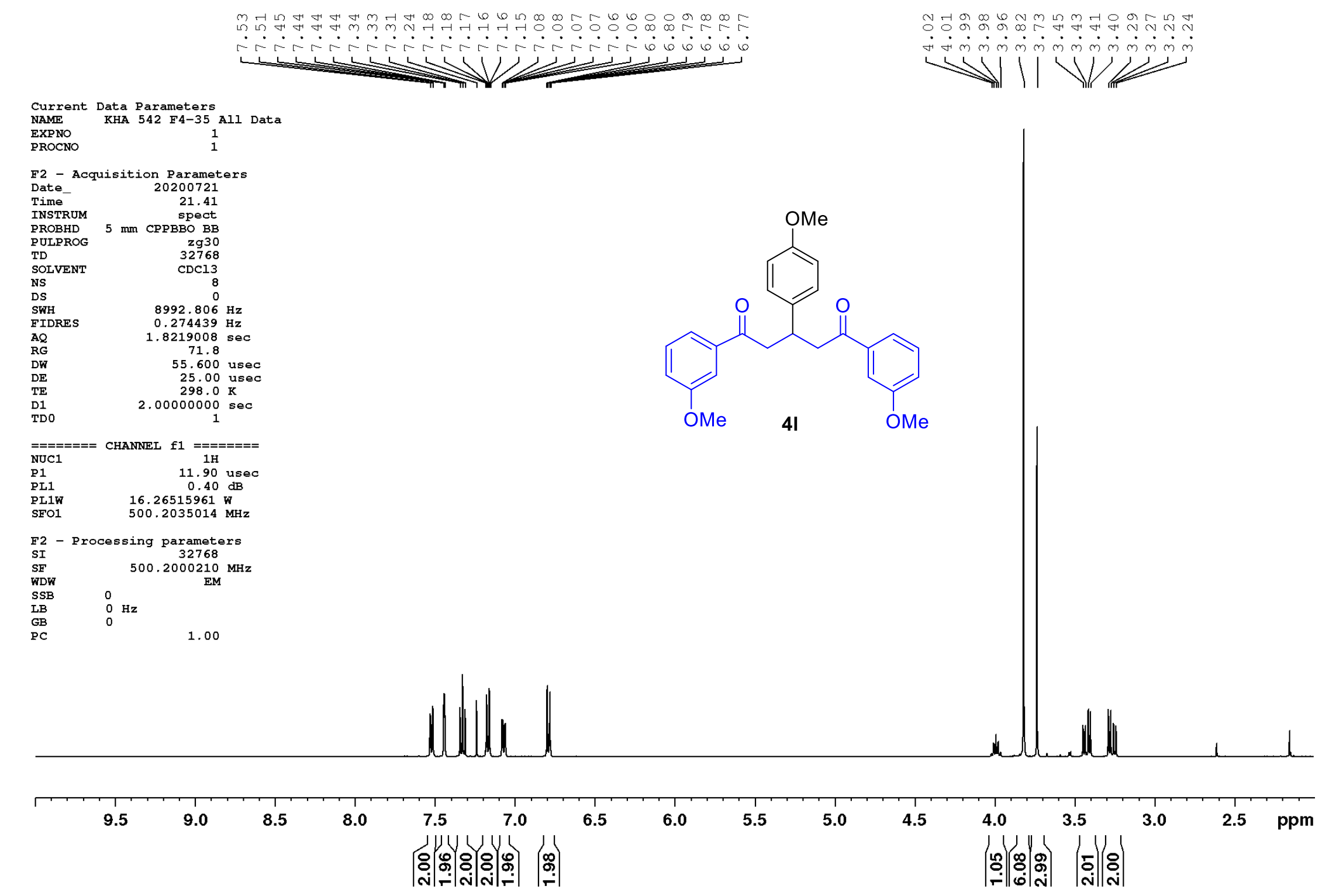

Figure S68. ${ }^{1} \mathrm{H}$ NMR spectrum of compound $41\left(500 \mathrm{MHz}, \mathrm{CDCl}_{3}, 25{ }^{\circ} \mathrm{C}\right)$ 
Current Data Parameters
NAME KHA 542 F4-35 All Data
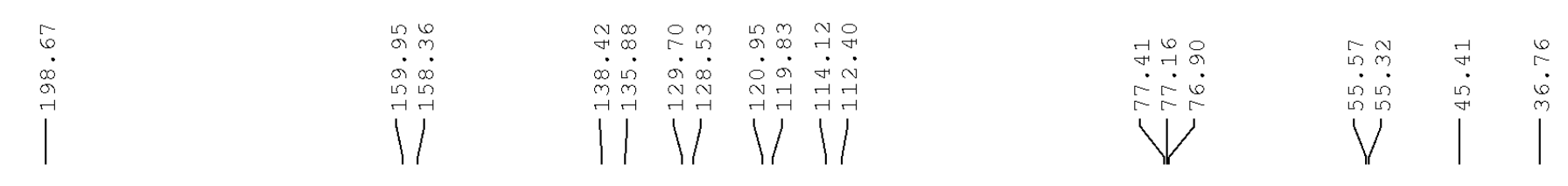

NAME KHA 542 F4-35 All Data

PROCNO
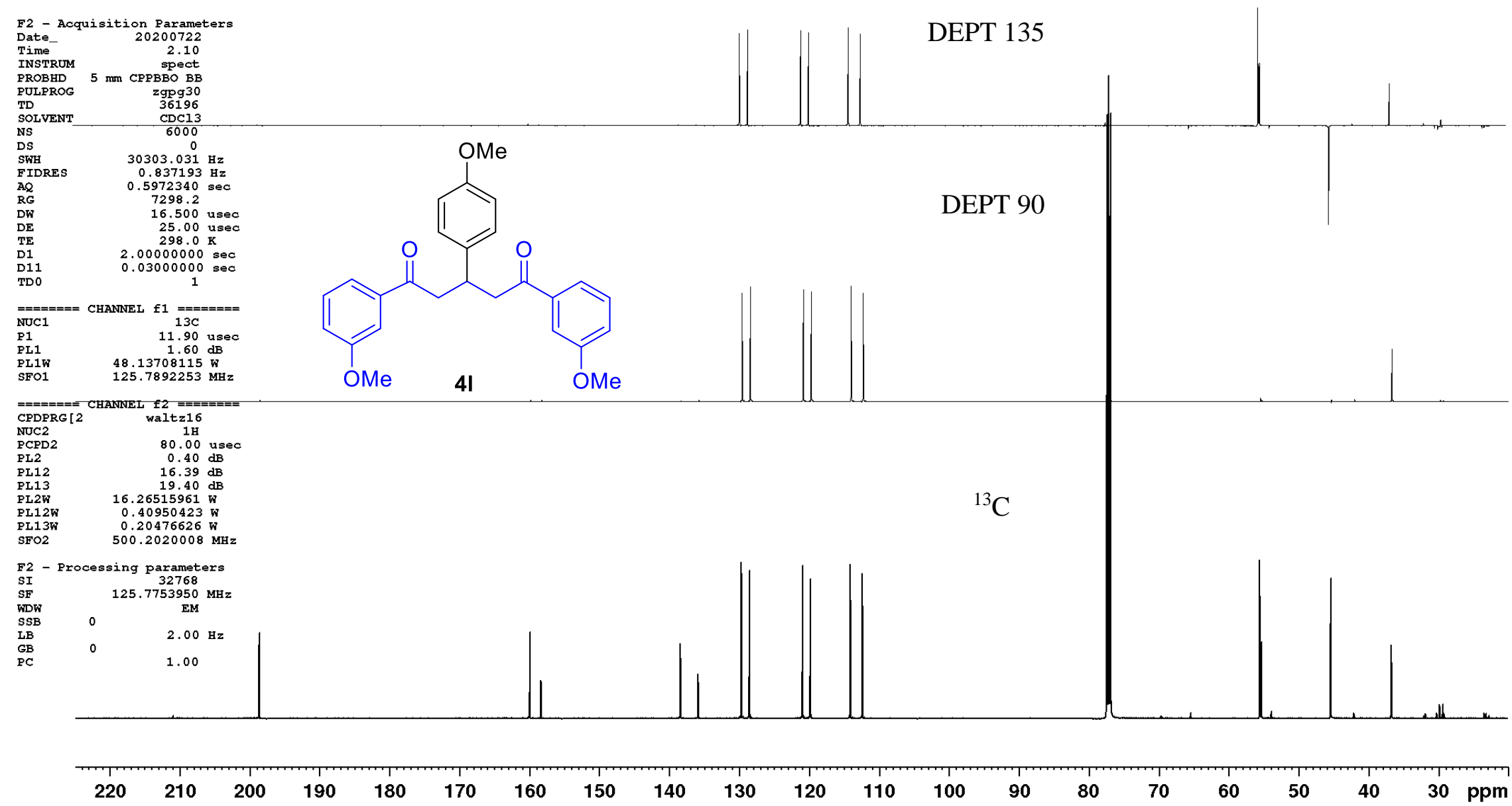

Figure S69. ${ }^{13} \mathrm{C}$ NMR spectrum of compound $4 \mathbf{l}\left(125 \mathrm{MHz}, \mathrm{CDCl}_{3}, 25{ }^{\circ} \mathrm{C}\right)$ 
Current Data Parameters
NAME KHA 544 F4-30 All Data

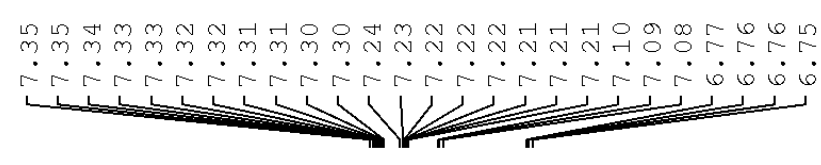

EXPNO
PROCNO

F2 - Acquisition Parameters

$\begin{array}{lr}\text { Date } & 20200724 \\ \text { Time } & 22.26\end{array}$

spect
INSTRUM

$\begin{array}{rr}\text { PROBHD } & 5 \mathrm{~mm} \text { PABBO } \mathrm{BB} / \\ \mathrm{PULPROG} & \mathrm{zg} 30\end{array}$

TD 32768

$\begin{array}{lr}\text { SOLVENT } & \mathrm{CDCl} \\ \text { NS } & 8 \\ \text { DS } & 0\end{array}$

SWH $\quad 9009.009 \mathrm{~Hz}$

$\begin{array}{lr}\text { FIDRES } & 0.274933 \mathrm{~Hz} \\ \text { AQ } & 1.8186240 \mathrm{sec}\end{array}$

$\begin{array}{lr}\mathrm{DW} & 126.44 \\ \mathrm{DE} & 55.500 \text { usec } \\ \mathrm{L} & 7.62 \text { usec }\end{array}$

TE $298.0 \mathrm{~K}$

TD0

$=======\begin{gathered}\text { CHANNEL } f 1 \quad======= \\ 500.1335009 \mathrm{MHz}\end{gathered}$
SFO1

NUC1 14.00 usec

$149911 \mathrm{~W}$

F2 - Processing parameters

SI $\quad 32768$

$\begin{array}{ll}\text { SF } & 500.1300224 \mathrm{MHz}\end{array}$

$\begin{array}{ll}\text { SSB } & 0 \\ \text { LB } & 0 \\ \text { HZ }\end{array}$

$\begin{array}{lll}\mathrm{GB} & 0 & \\ \mathrm{PC} & & \end{array}$
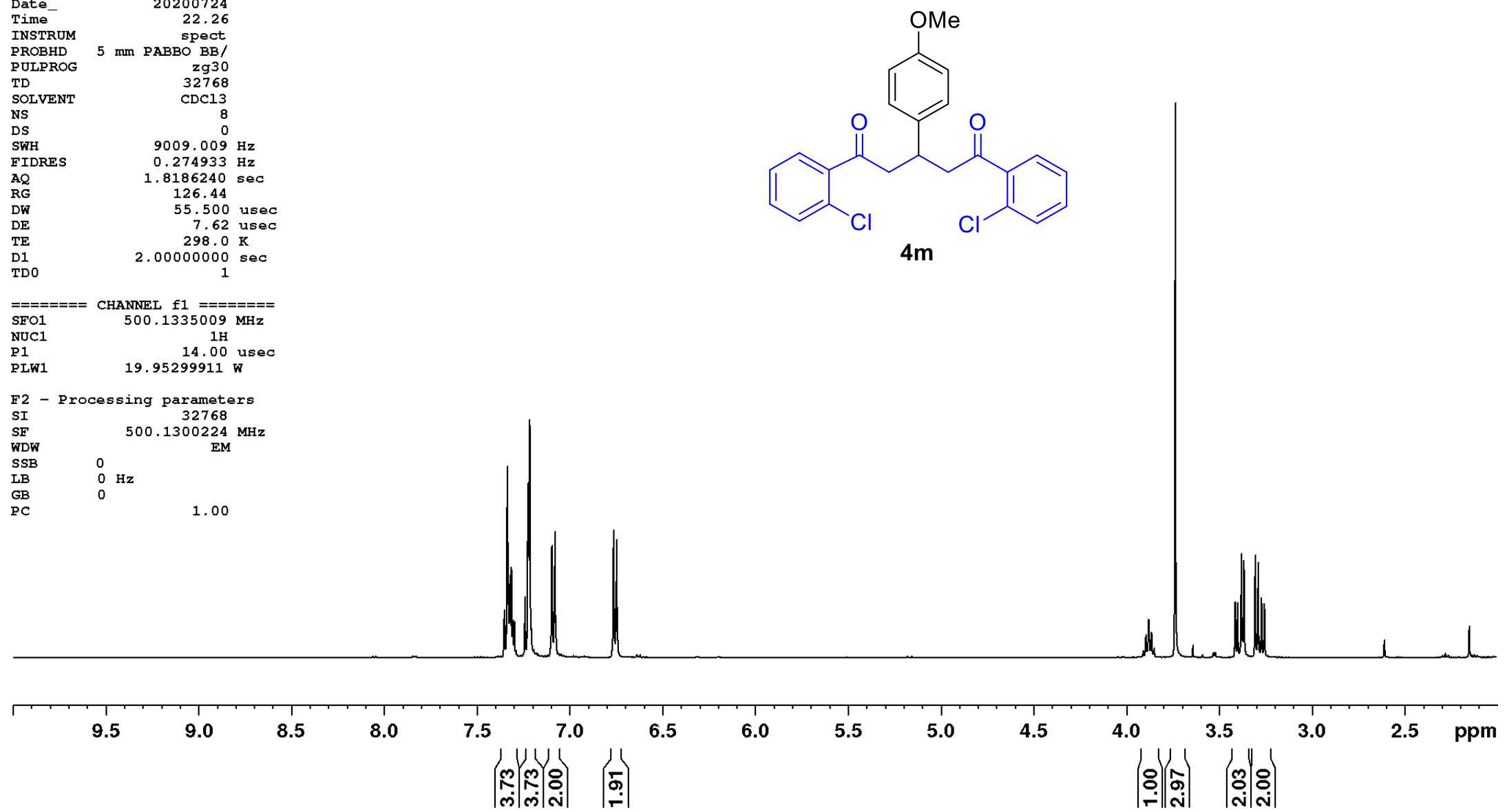

Figure S70. ${ }^{1} \mathrm{H} \mathrm{NMR}$ spectrum of compound $\mathbf{4 m}\left(500 \mathrm{MHz}, \mathrm{CDCl}_{3}, 25{ }^{\circ} \mathrm{C}\right)$ 


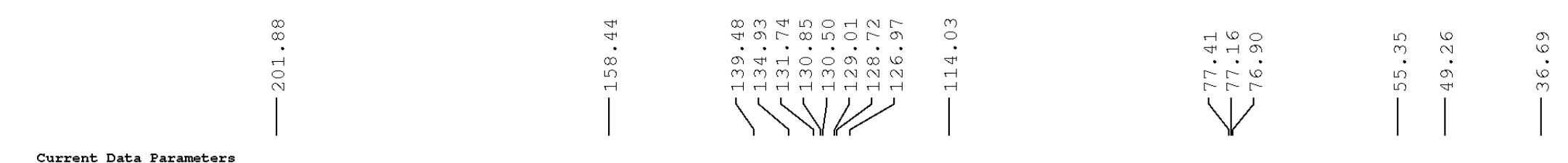

Current Data Parameters
NAME KHA 544 F4-30 All Data

EXPNO

F2 - Acquisition Parameters
Date_ 20200725

\section{DEPT 135}$$
\text { INSTR }
$$$$
\begin{array}{r}
\text { spect } \\
\text { PROBHD } 5 \mathrm{~mm} \text { PABBO BB }
\end{array}
$$

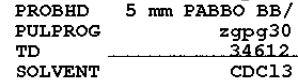$$
\begin{array}{lr}
\text { SOLVENT } & 34612 \\
\text { NS } & \text { CDC13 } \\
\text { DS } & 8000 \\
\text { SWH } & 0
\end{array}
$$

$\begin{array}{lc}\text { DS } & 0 \\ \text { SWH } & 28846.154 \mathrm{~Hz} \\ \text { FIDRES } & 0.833415 \mathrm{~Hz}\end{array}$

\begin{tabular}{lr} 
FIDRES & $28846.154 \mathrm{~Hz}$ \\
AQ & $0.833415 \mathrm{~Hz}$ \\
RG & $0.599913 \mathrm{sec}$ \\
DW & 200.35 \\
\hline
\end{tabular}

200.35
17.333 usec
6.50 usec

$\begin{array}{lr}\text { DE } & 69.50 \mathrm{usec} \\ \text { TE } & 2.00000000 \mathrm{~K} \\ \text { D1 } & 0.03000000 \mathrm{sec} \\ \text { D11 } & 1\end{array}$

$=======$ CHANNEL $\mathrm{f1}=======$
$\mathrm{SFO1}$
$125.7716219 \mathrm{MHz}$

$\begin{array}{lr}\text { SFO1 } & 125.7716219 \mathrm{MHz} \\ \text { NUC1 } & 13 \mathrm{C} \\ \text { P1 } & 10.75 \text { usec } \\ \text { PLW1 } & 50.11899948 \mathrm{w}\end{array}$

$\begin{array}{lr}\text { PLW1 } & 50.11899948 \mathrm{~W} \\ ======= & \text { CHANNEL } f 2======= \\ \text { SFO2 } & 500.1320005 \mathrm{MHz}\end{array}$

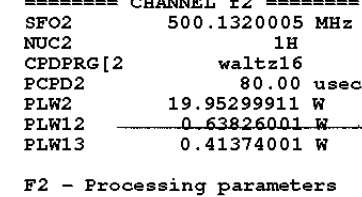

F2 - Processing parameter

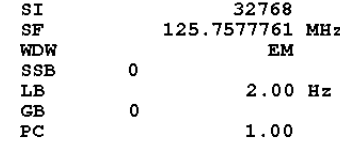
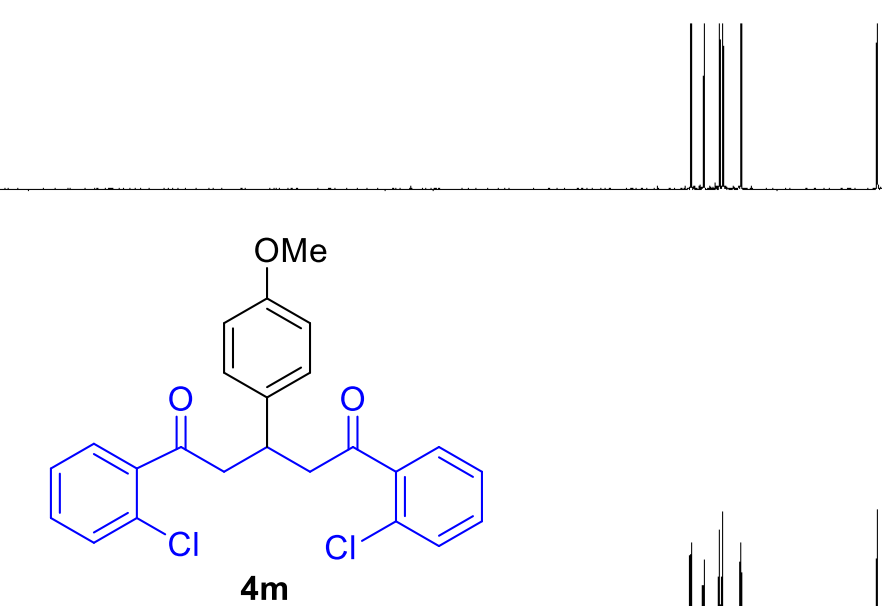

DPT 135

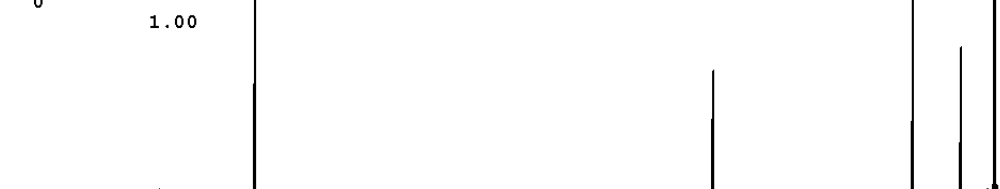

$220 \quad 210$

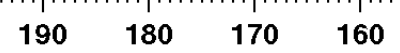

150

$140 \quad 130$

120

100

80

Figure S71. ${ }^{13} \mathrm{C}$ NMR spectrum of compound $\mathbf{4 m}\left(125 \mathrm{MHz}, \mathrm{CDCl}_{3}, 25{ }^{\circ} \mathrm{C}\right)$ 
Current Data Parameters

All Data

EXPNO

F2 - Acquisition Parameters

Date
Time

20200722
21.01

INSTRUM

PROBHD $5 \mathrm{~mm}$ PABBO BB/

PULPROG $\quad$ zg30

TD

SOLVEN

NS

SWH

FIDRE
AQ
RG
DW

$\mathrm{DW}$
$\mathrm{DE}$

TE

TDO

$\mathrm{CDCl} 3$
8

$9009.009 \mathrm{~Hz}$

$0.274933 \mathrm{~Hz}$

$1.8186240 \mathrm{sec}$
98.66

55.500 usec

55.500 usec
7.62 usec

$\begin{aligned} & 298.0 \mathrm{~K} \\ & 2.00000000 \mathrm{sec}\end{aligned}$

$=======$ CHANNEL $\mathrm{f} 1 \quad=======$

SFO1 $\quad 500.1335009 \mathrm{MH}$

$\begin{array}{lr}\text { P1 } & 1 \mathrm{H} \\ & 14.00 \mathrm{usec}\end{array}$

SI Processing

$\begin{array}{lc}\text { SF } & 500.1300221 \mathrm{MHz} \\ \text { WDW } & \text { EM }\end{array}$

$\begin{array}{llll}\mathrm{SSB} & 0 & \\ \mathrm{LB} & 0 \mathrm{~Hz} & \\ \mathrm{~GB} & 0 & \\ \mathrm{PC} & & 1.00\end{array}$
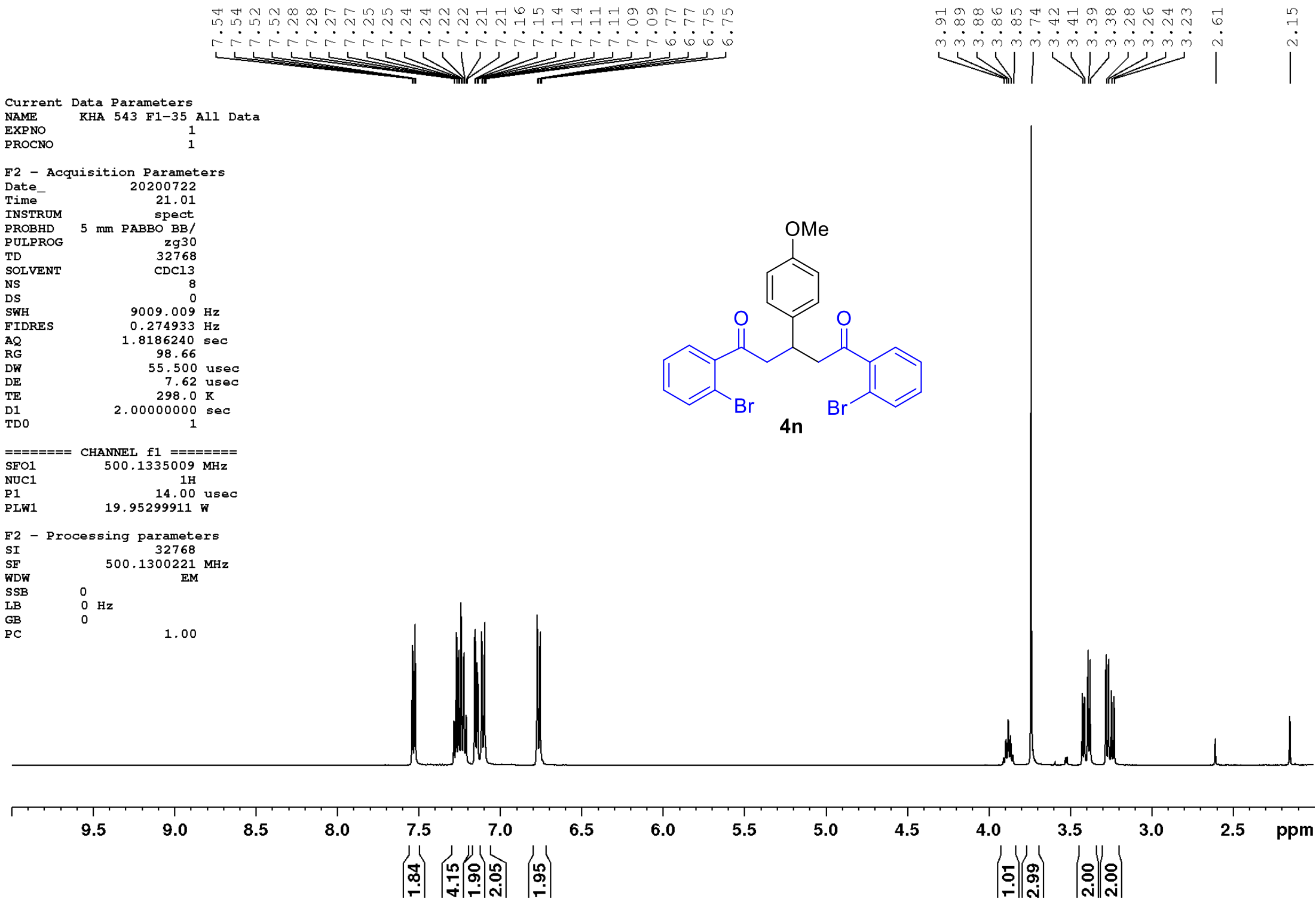

Figure S72. ${ }^{1} \mathrm{H}$ NMR spectrum of compound 4 n $\left(500 \mathrm{MHz}, \mathrm{CDCl}_{3}, 25{ }^{\circ} \mathrm{C}\right)$ 


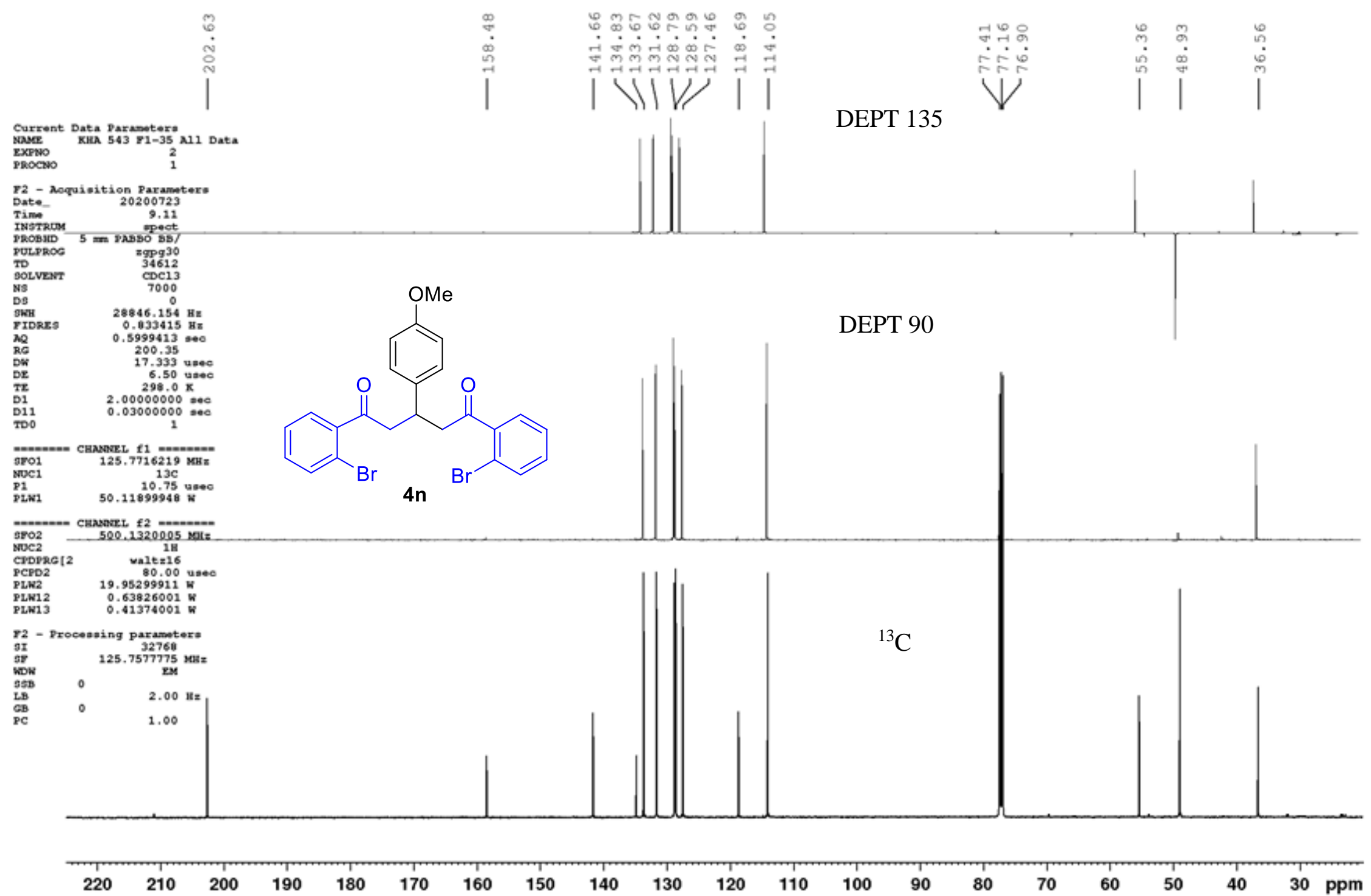

Figure S73. ${ }^{13} \mathrm{C}$ NMR spectrum of compound 4 n $\left(125 \mathrm{MHz}, \mathrm{CDCl}_{3}, 25{ }^{\circ} \mathrm{C}\right)$ 
Current Data Parameters

NAME KHA 546 F44-57 a11 Data

EXPNO
PROCNO

F2 - Acquisition Parameters

$\begin{array}{lr}\text { Date } & 20200728 \\ \text { Time } & 22.09\end{array}$

INSTRUM
PROBHD $5 \mathrm{~mm}$ PABBO BB/

$\begin{array}{lr}\text { PULPROG } & \mathrm{zg} 30 \\ \text { TD } & 32768\end{array}$

NS

$\begin{array}{lc}\text { DS } & 0 \\ \text { SWH } & 9009.009 \mathrm{~Hz} \\ \text { FIDRES } & 0.274933 \mathrm{~Hz}\end{array}$

$\begin{array}{lr}\text { FIDRES } & 0.274933 \mathrm{~Hz} \\ \text { AQ } & 1.8186240 \mathrm{sec}\end{array}$

$\begin{array}{lr}\text { RG } & 112.51 \\ \text { DW } & 55.500 \text { usec }\end{array}$

$\begin{array}{lr}\mathrm{DW} & 55.500 \text { usec } \\ \mathrm{DE} & 7.62 \text { usec } \\ \mathrm{TE} & 299.3 \mathrm{~K}\end{array}$

D1 $\quad 2.00000000 \mathrm{Kec}$

TDO

$=======\begin{gathered}\text { CHANNEL } f 1 \quad======= \\ 500.1335009 \mathrm{MHz}\end{gathered}$
SFO1

NUC1 14.00 use

$\begin{array}{lr}\text { P1 } & 14.00 \text { usec } \\ \text { PLW1 } & 19.95299911 \mathrm{~W}\end{array}$

F2 - Processing parameters

$\begin{array}{lc}\text { SI } & 32768 \\ \text { SF } & 500.1300224 \mathrm{MHz}\end{array}$

WDW

$\begin{array}{ll}\text { SSB } & 0 \\ \text { LB } & 0 \\ \text { GB } & 0\end{array}$

PC $\quad 1.00$
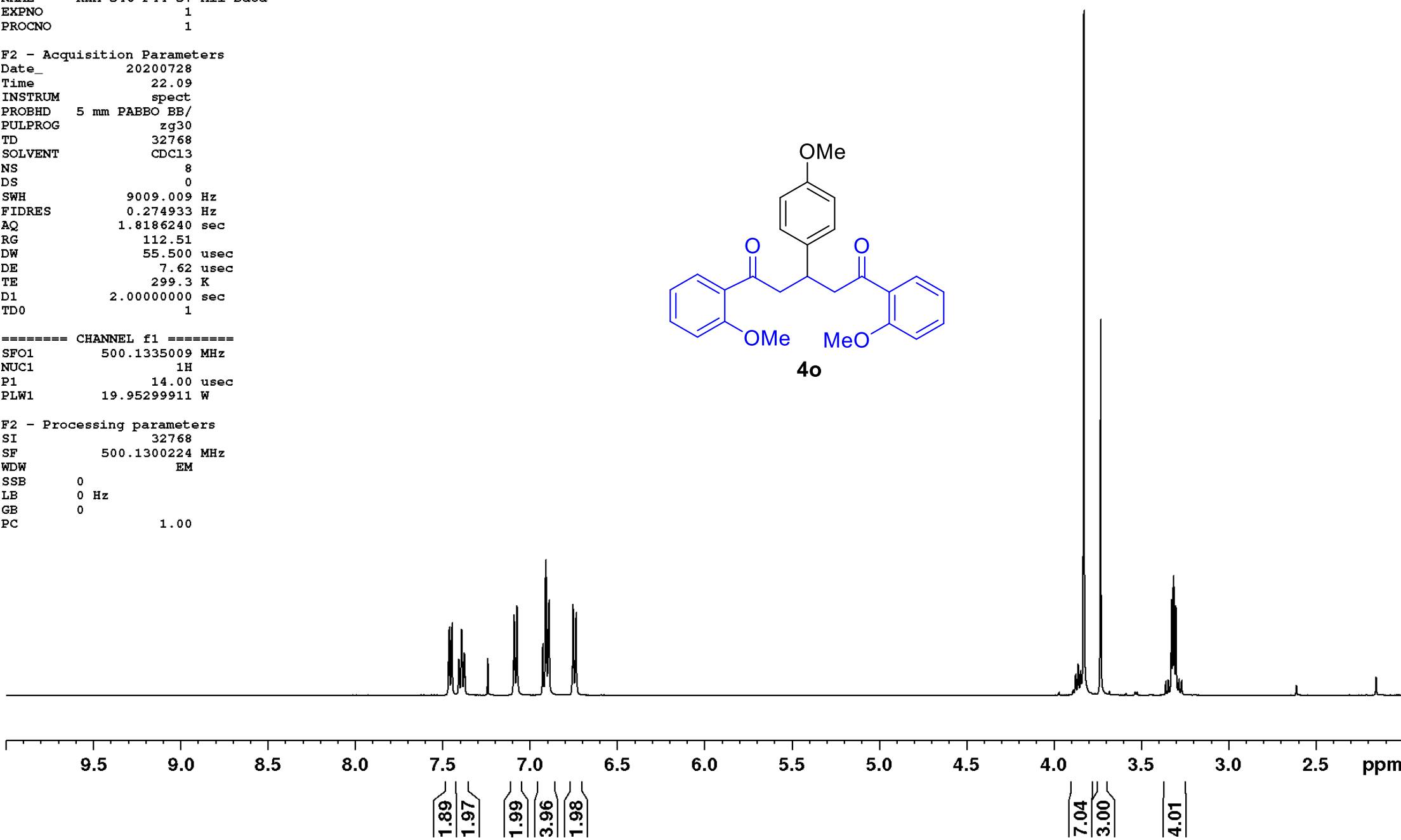

Figure S74. ${ }^{1} \mathrm{H}$ NMR spectrum of compound $40\left(500 \mathrm{MHz}, \mathrm{CDCl}_{3}, 25{ }^{\circ} \mathrm{C}\right)$ 


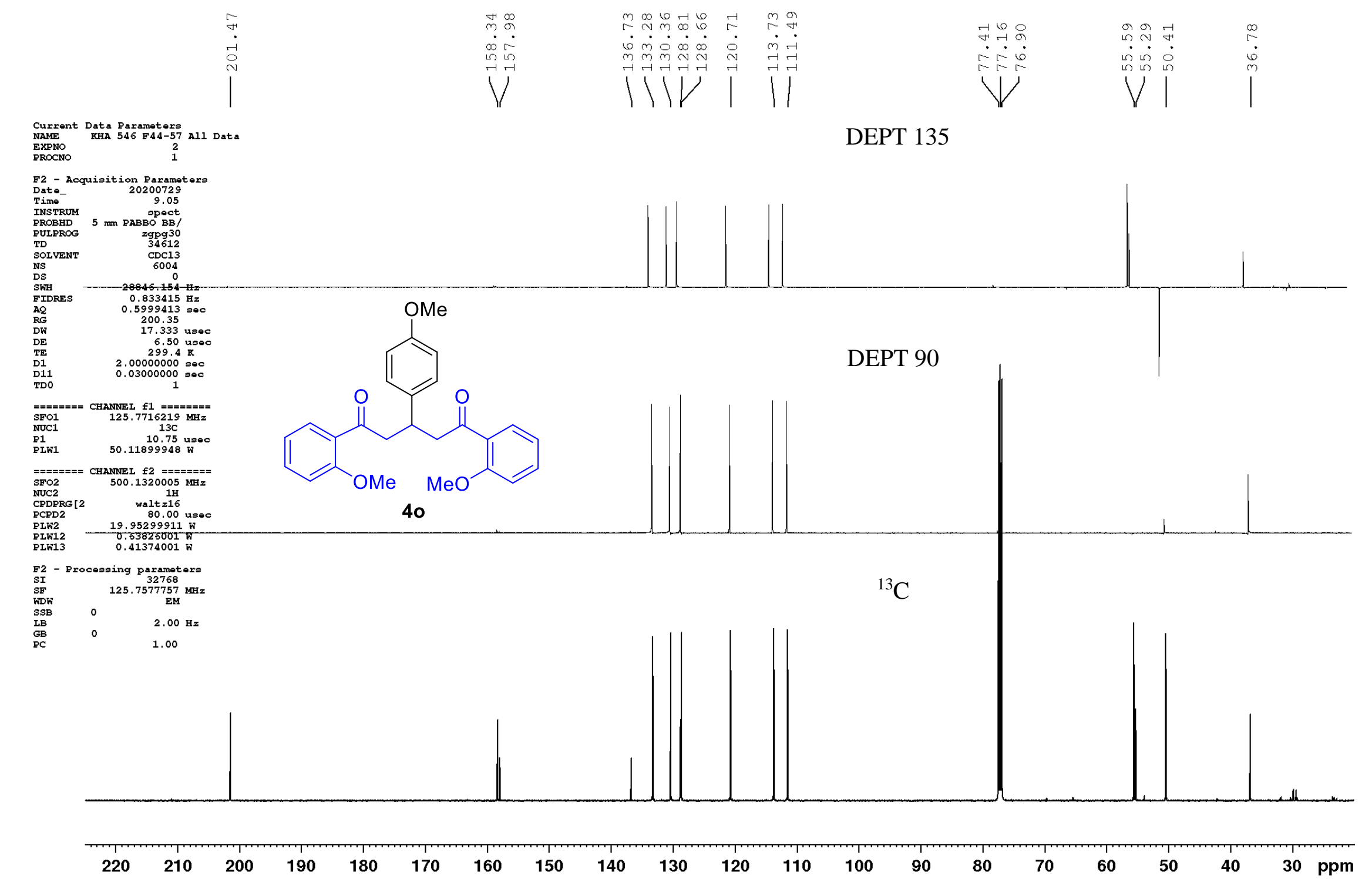

Figure S75. ${ }^{13} \mathrm{C}$ NMR spectrum of compound $40\left(125 \mathrm{MHz}, \mathrm{CDCl}_{3}, 25{ }^{\circ} \mathrm{C}\right)$ 
Current Data Parameters

PROCNO

1

F2 - Acquisition Parameters

Date_ 20200806

21.27
INSTRUM

SOLVENT

NS

DS
SWH
FIDRES
AQ
RG
DG

RG

$\mathrm{DW}$
$\mathrm{DE}$
$\mathrm{TE}$

TD0

$==\mathrm{cHantath}$

NUC1 $===$ CHANNEL $\mathrm{f} 1 \mathrm{=}======$
P1

$\begin{array}{lrl}\text { P1 } & 11.90 \text { use } \\ \text { PL1 } & 0.40 \mathrm{~dB}\end{array}$

$\begin{array}{ll}\text { PL1W } & 16.26515961 \mathrm{~W} \\ \text { SFO1 } & 500.2035014 \mathrm{MHz}\end{array}$

F2 - Processing parameters

$\begin{array}{ll}\text { SI } & 500.2000207 \\ \text { WDW } & \text { MHz }\end{array}$

SSB 0

$\begin{array}{lll}\mathrm{LB} & 0 \mathrm{~Hz} & \\ \mathrm{~GB} & 0^{\mathrm{Hz}} & \\ \mathrm{PC} & & 1.00\end{array}$
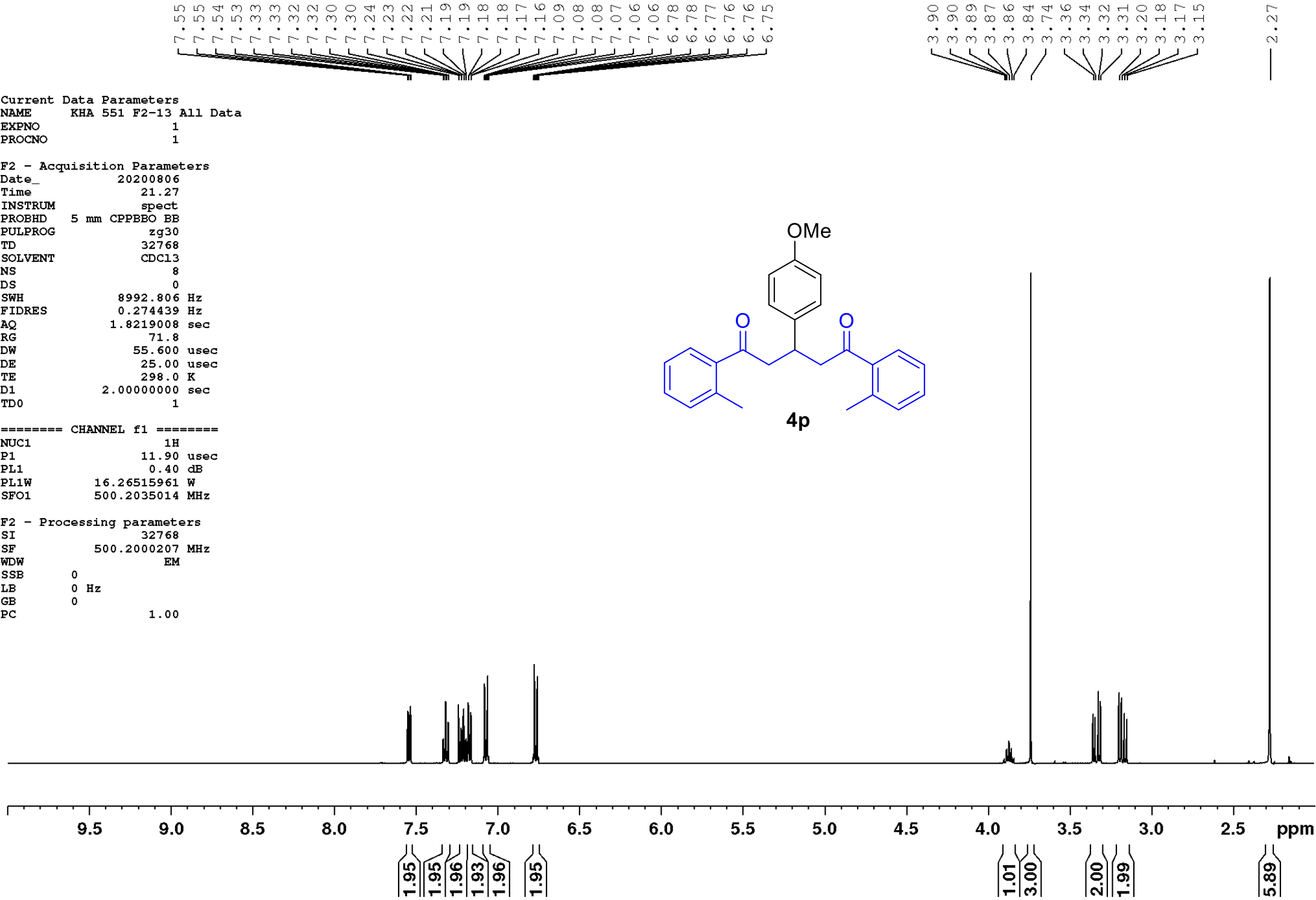

Figure S76. ${ }^{1} \mathrm{H}$ NMR spectrum of compound $4 \mathbf{p}\left(500 \mathrm{MHz}, \mathrm{CDCl}_{3}, 25{ }^{\circ} \mathrm{C}\right)$ 

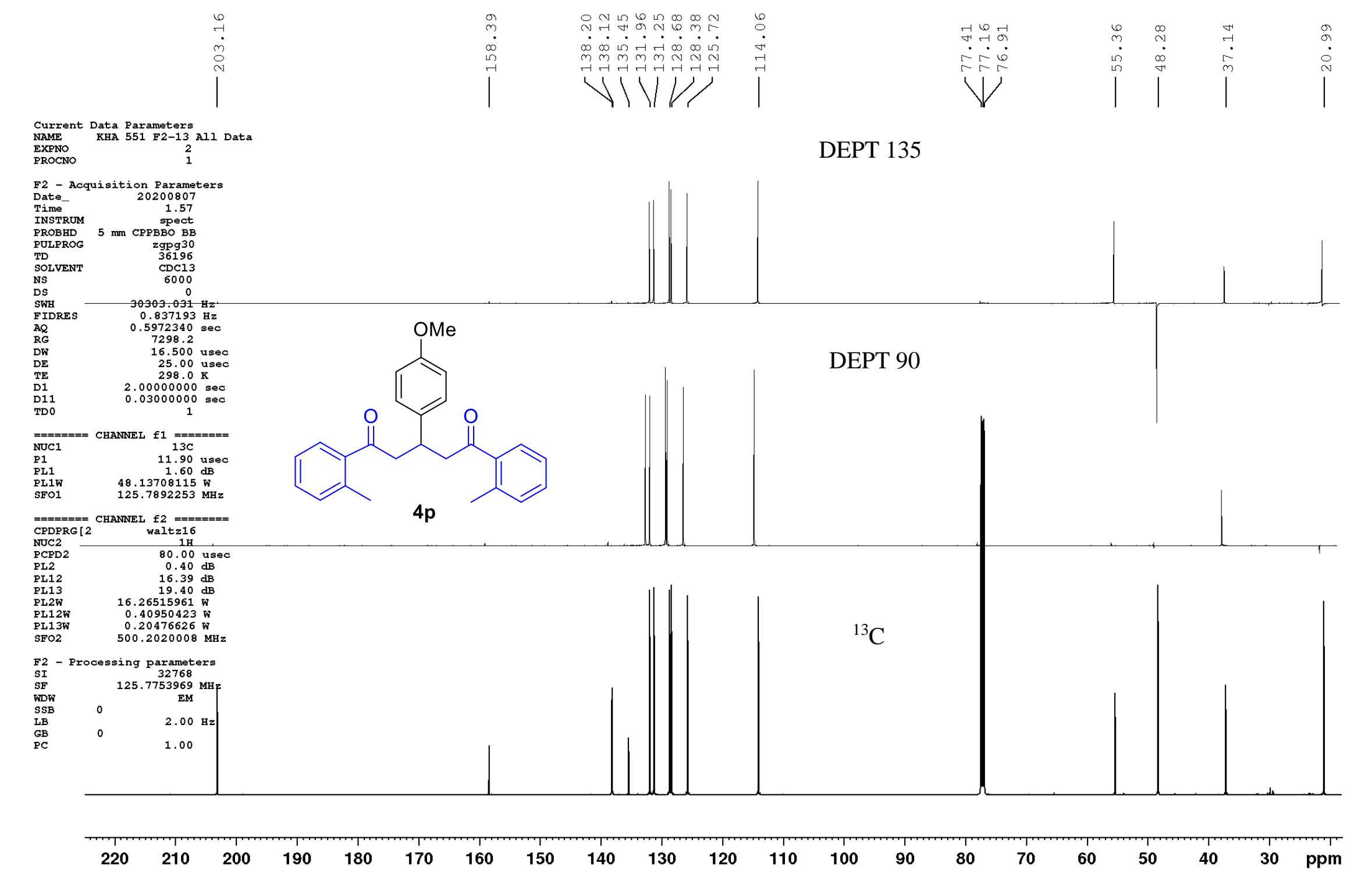

Figure S77. ${ }^{13} \mathrm{C}$ NMR spectrum of compound $4 \mathbf{p}\left(125 \mathrm{MHz}, \mathrm{CDCl}_{3}, 25{ }^{\circ} \mathrm{C}\right)$ 
Current Data Parameters
NAME

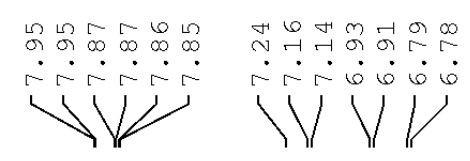

$$
\begin{aligned}
& \begin{array}{l}
\text { EXPNO } \\
\text { PROCNO }
\end{array} \\
& 1
\end{aligned}
$$
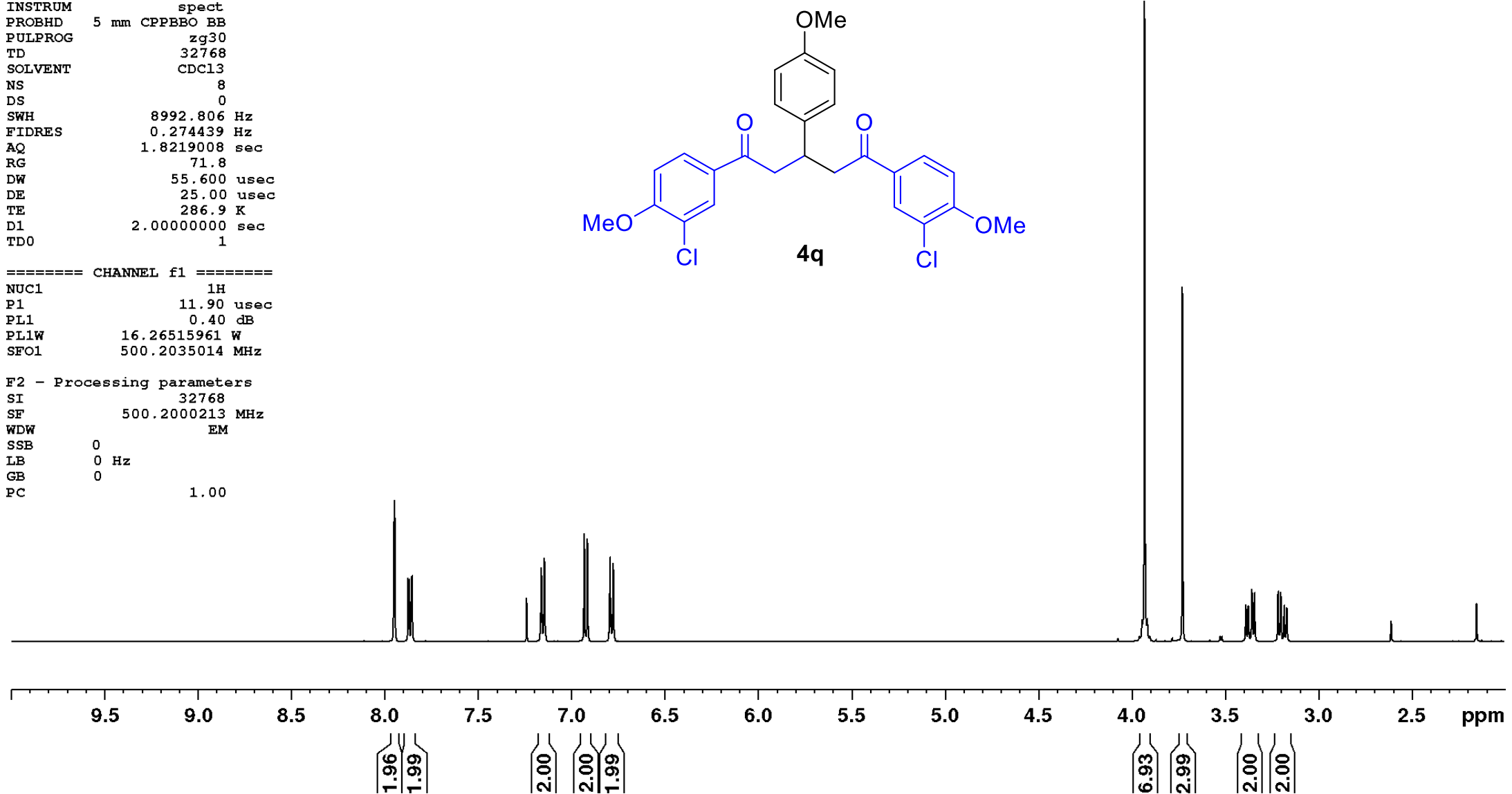

Figure S78. ${ }^{1} \mathrm{H}$ NMR spectrum of compound $\mathbf{4 q}\left(500 \mathrm{MHz}, \mathrm{CDCl}_{3}, 25{ }^{\circ} \mathrm{C}\right)$ 


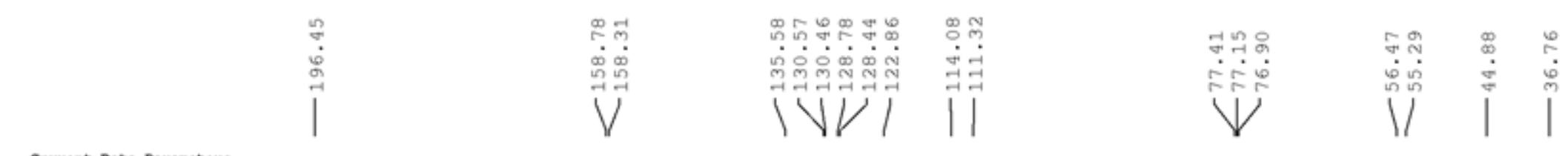

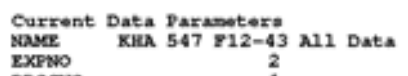

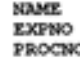

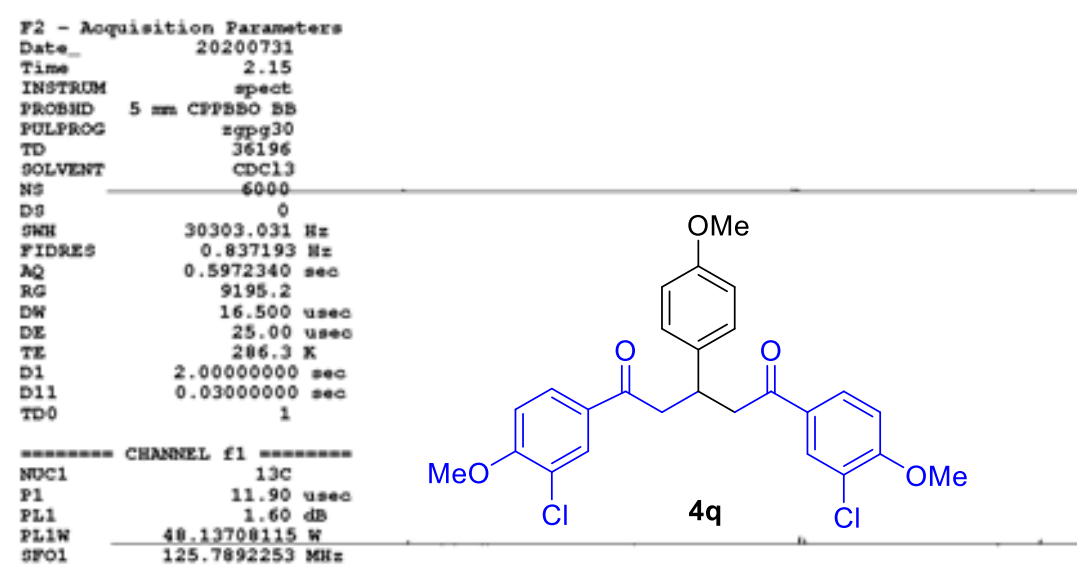

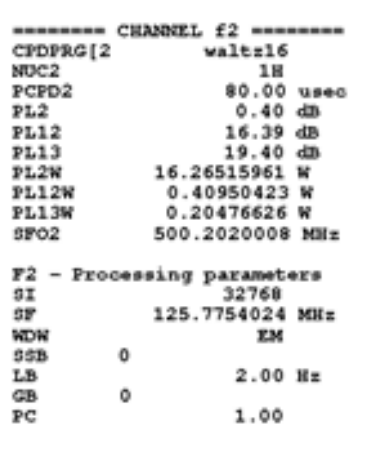

DEPT 135
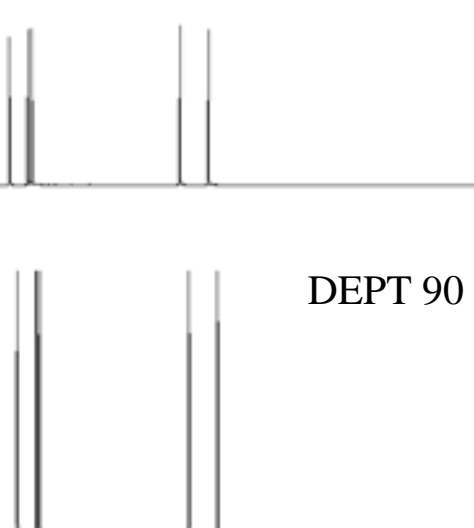

DEPT 90
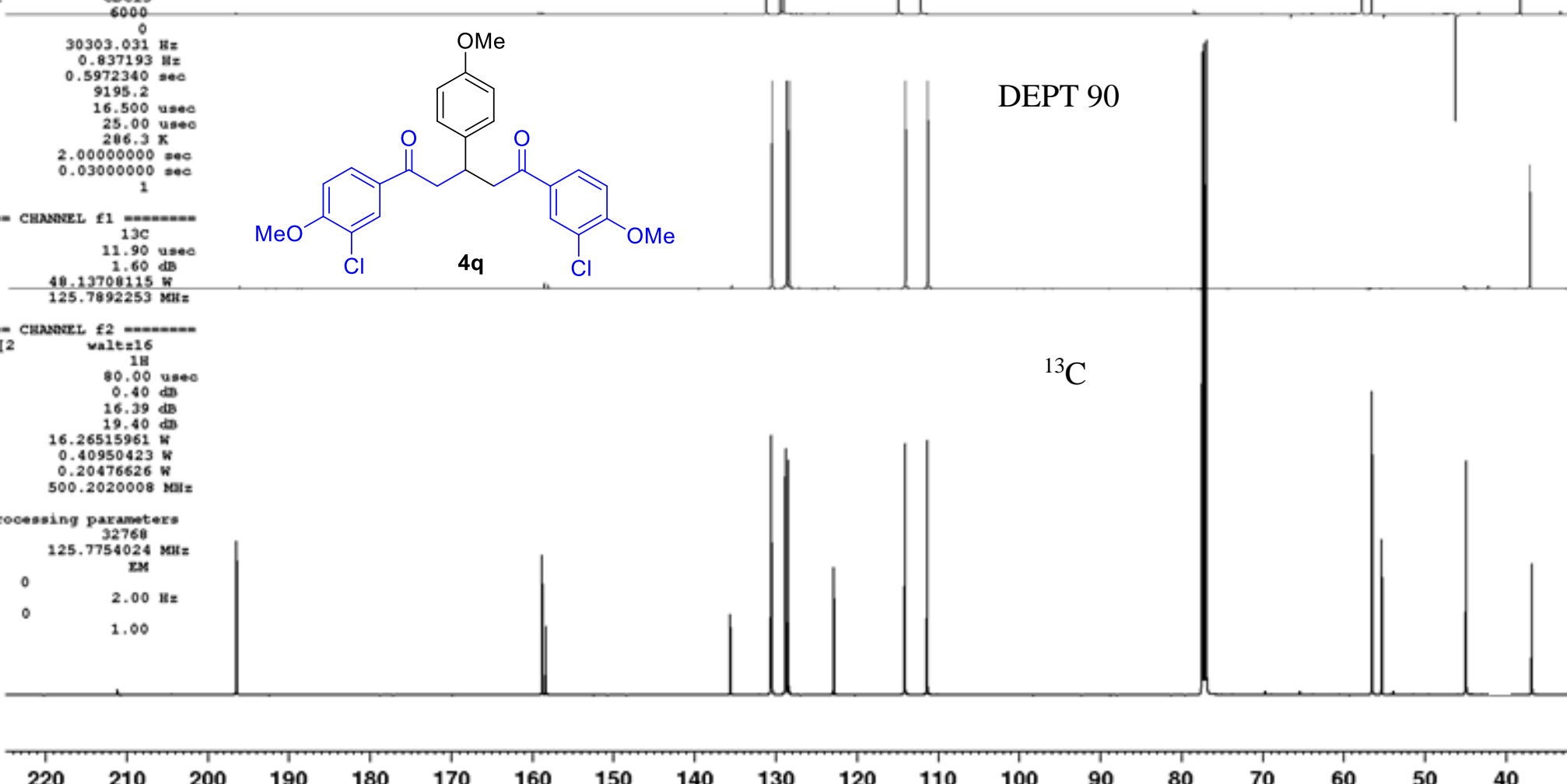

190

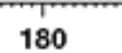

170160
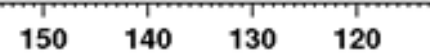

110100

90
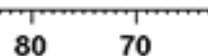

$60 \quad 50$

$4030 \mathrm{ppm}$

Figure S79. ${ }^{13} \mathrm{C}$ NMR spectrum of compound $4 q\left(125 \mathrm{MHz}, \mathrm{CDCl}_{3}, 25{ }^{\circ} \mathrm{C}\right)$ 
Current Data Parameters
NAME KHA 545 F4-25 All Data

KHA 545 F $4-25$

PROCNO

F2 2 - Acquisition Parameters
Date_ 20200726

Time

spect
PROBHD $5 \mathrm{~mm}$ PABBO BB/

PULPROG

TD

SOLVE

NS $\quad 16$

$\begin{array}{ll}\text { DS } & 00 \\ \text { SWH } & 9009.009 \mathrm{~Hz}\end{array}$

FIDRES $\quad 0.274933 \mathrm{~Hz}$

$\begin{array}{lr}\mathrm{AQ} & 1.8186240 \\ \mathrm{RG} & 179.67\end{array}$

$\begin{array}{rr}\mathrm{DW} & 55.500 \text { usec } \\ \mathrm{DE} & 7.62 \text { usec }\end{array}$

$\begin{array}{lr}\mathrm{TE} & 298.0 \mathrm{~K} \\ \mathrm{D} 1 & 2.00000000 \mathrm{sec}\end{array}$

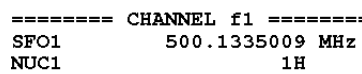

$\begin{array}{lr}\text { P1 } & 14.00 \text { usec } \\ \text { PLW1 } & 19.95299911 \mathrm{~W}\end{array}$

F2 - Processing parameters

$\begin{array}{ll}\text { SI } & 32768 \\ \text { SF } & 500.1300229 \mathrm{MHz}\end{array}$

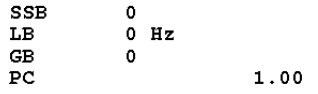
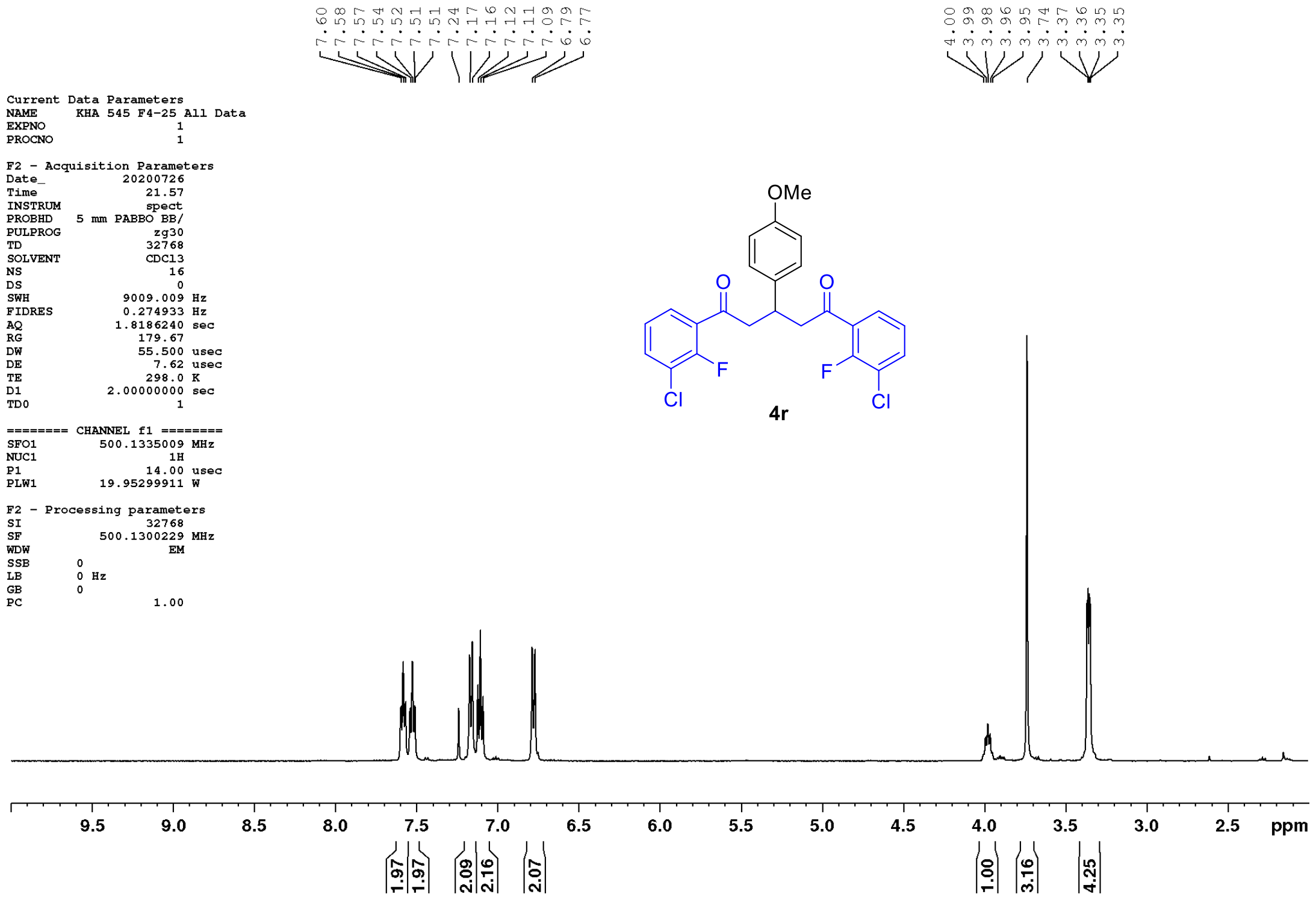

Figure S80. ${ }^{1} \mathrm{H}$ NMR spectrum of compound $4 \mathbf{r}\left(500 \mathrm{MHz}, \mathrm{CDCl}_{3}, 25{ }^{\circ} \mathrm{C}\right)$ 


\section{Current Data Parameters}
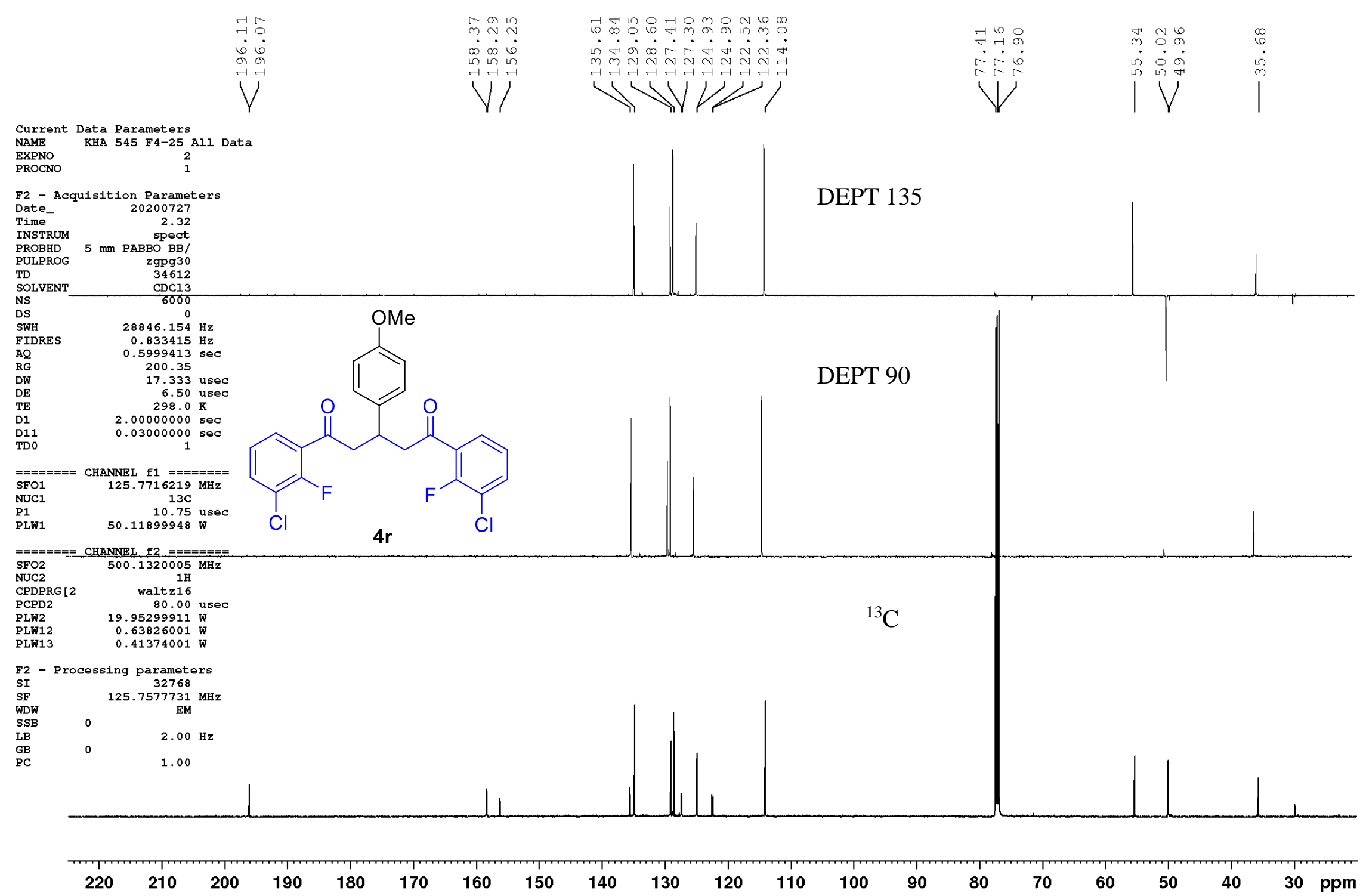

Figure S81. ${ }^{13} \mathrm{C}$ NMR spectrum of compound $4 \mathbf{r}\left(125 \mathrm{MHz}, \mathrm{CDCl}_{3}, 25{ }^{\circ} \mathrm{C}\right)$ 
Current Data Parameters
NAME KHA 549 F11-24 All data-AV400

EXPNO

F2 - Acquisition Parameters

$\begin{array}{lr}\text { Date } & 20200801 \\ \text { Time } & 17.43\end{array}$

INSTRUM

PROBHD $5 \mathrm{~mm}$ Dual $13 \mathrm{c} /$

PULPROG

TD

SOLVE

NS

SWH
FIDRES

AQ

RG

$\mathrm{DE}$
$\mathrm{DE}$
$\mathrm{T} 1$
$\mathrm{D} 1$

69.600 usec
6.50 usec

$2.00000000 \mathrm{sec}$

$=======$ CHANNEI $\mathrm{f} 1$

P1 1

11.30 usec
$-2.00 \mathrm{~dB}$

SFO1 $400.1328009 \mathrm{MHz}$

F2 - Processing parameters

SI $\quad 16384$

$\begin{array}{lr}\text { SF } & 400.1300171 \\ \text { WDW } & \text { MHz }\end{array}$

$\begin{array}{ll}\text { SSB } & 0 \\ \text { LB } & 0\end{array}$

$\begin{array}{lll}\mathrm{LB} & 0 \mathrm{~Hz} & \\ \mathrm{~GB} & 0 & \\ \mathrm{PC} & & 1.00\end{array}$
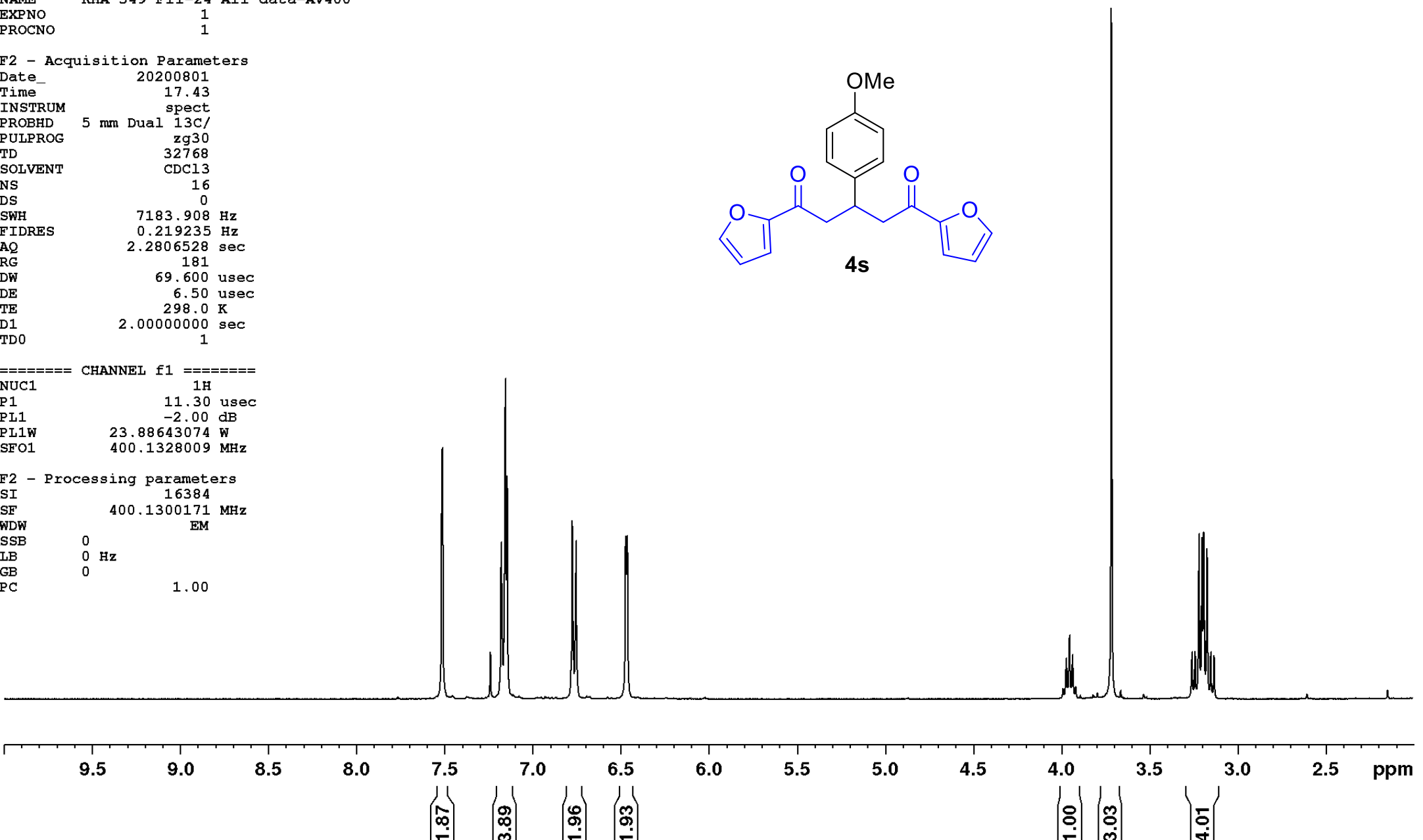

Figure S82. ${ }^{1} \mathrm{H}$ NMR spectrum of compound $4 \mathbf{s}\left(500 \mathrm{MHz}, \mathrm{CDCl}_{3}, 25{ }^{\circ} \mathrm{C}\right)$ 

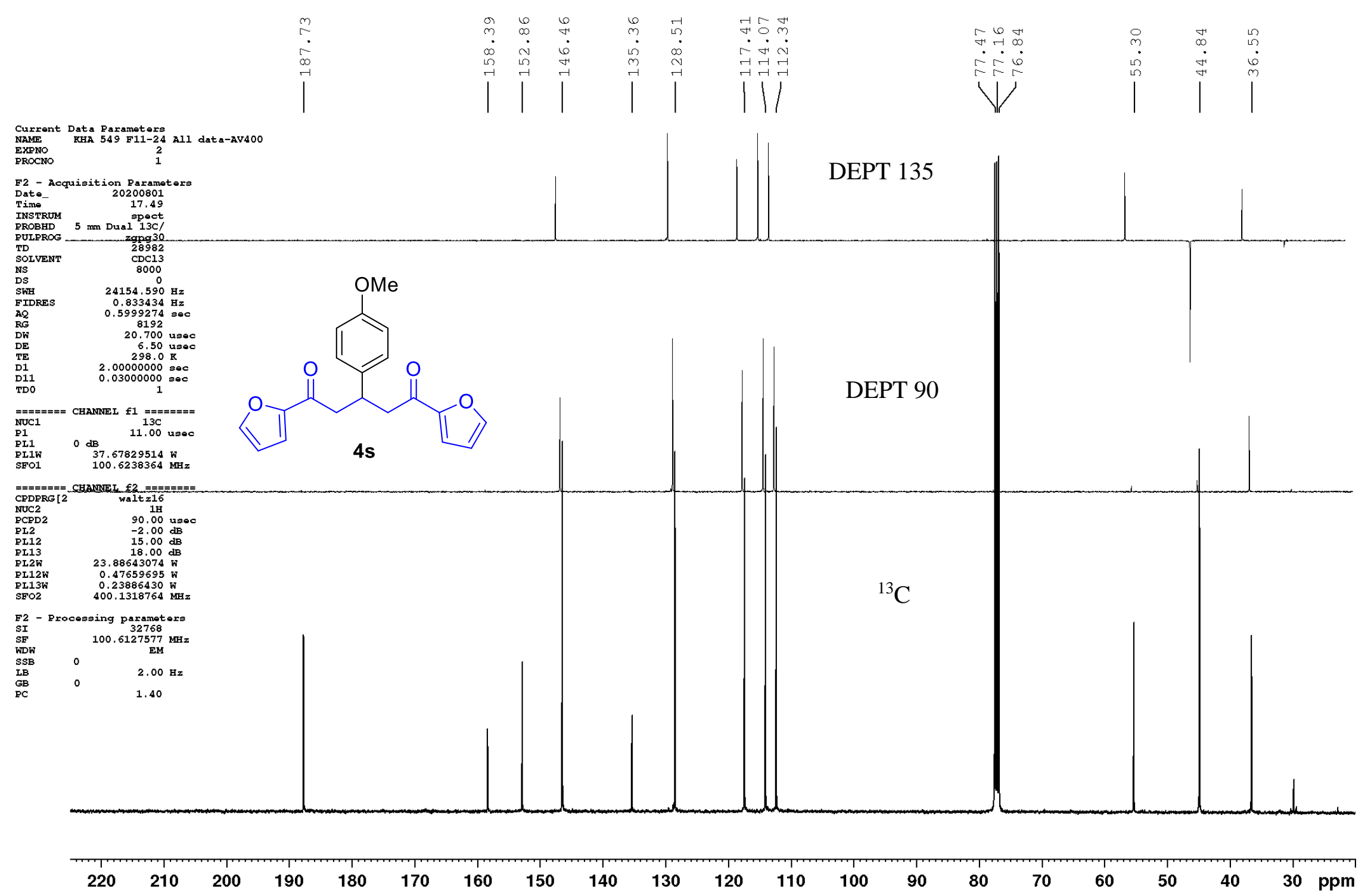

Figure S83. ${ }^{13} \mathrm{C}$ NMR spectrum of compound $4 \mathbf{s}\left(125 \mathrm{MHz}, \mathrm{CDCl}_{3}, 25{ }^{\circ} \mathrm{C}\right)$ 
Current Data Parameters
KHA 550 F4-23 All Data

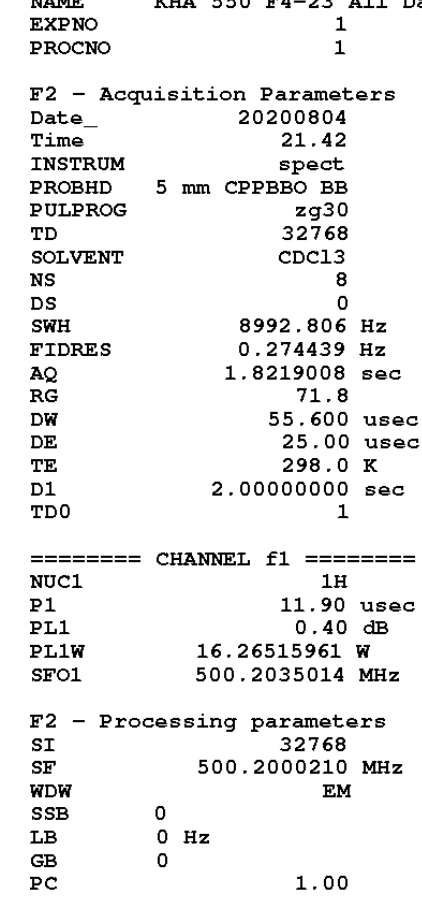

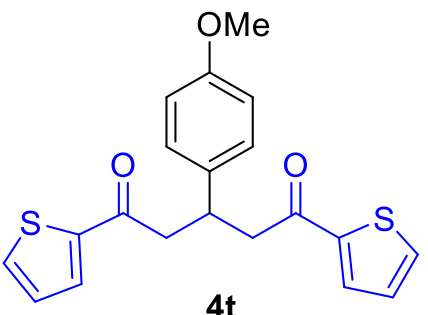

$4 t$

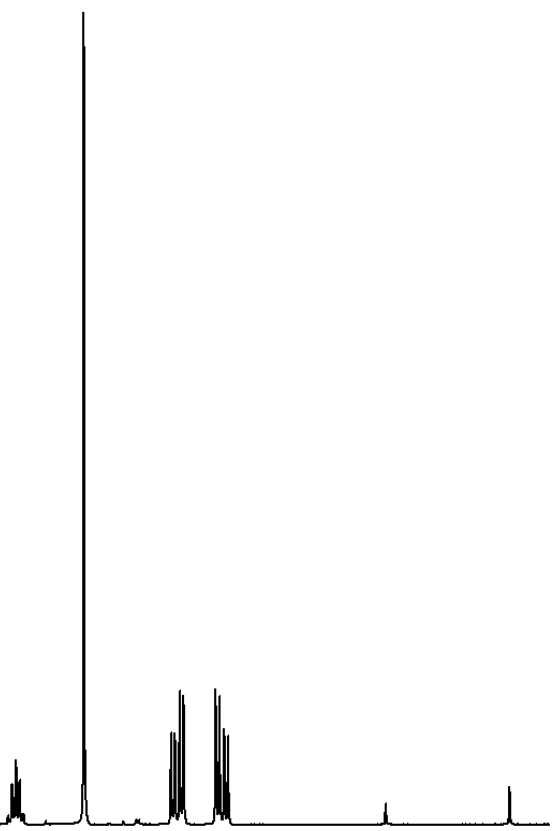

$\begin{array}{lll}9.5 & 9.0 & 8.5\end{array}$

8.0 ํㅜ||

6.0
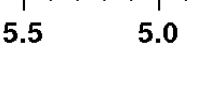

4.5

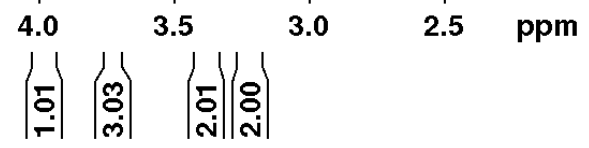

Figure S84. ${ }^{1} \mathrm{H} \mathrm{NMR}$ spectrum of compound $4 \mathbf{t}\left(500 \mathrm{MHz}, \mathrm{CDCl}_{3}, 25{ }^{\circ} \mathrm{C}\right)$ 


\section{Current Data Parameters
NAME}
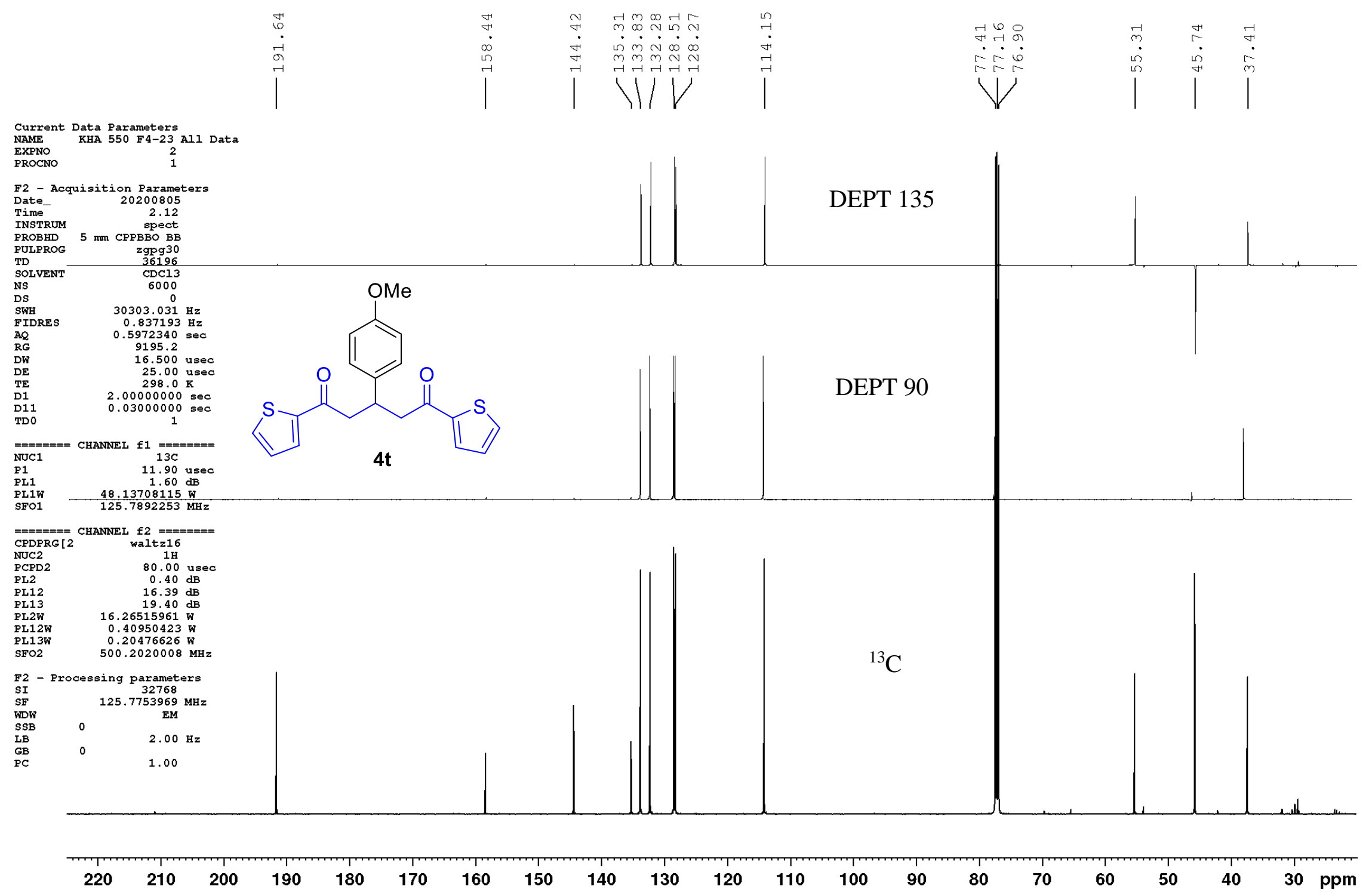

Figure S85. ${ }^{13} \mathrm{C}$ NMR spectrum of compound $4 \mathbf{t}\left(125 \mathrm{MHz}, \mathrm{CDCl}_{3}, 25{ }^{\circ} \mathrm{C}\right)$ 
Current Data Parameters
NAME KHA 552 F2-15 all data

$\begin{array}{lcl}\text { EXPNO } & 1 \\ \text { PROCNO } & 1\end{array}$

F2 - Acquisition Parameters

Time-

20200808
12.09

spect
PROBHD $5 \mathrm{~mm}$ PABBO BB/

PULPROG

TD

NS

DS

FIDRE

$\begin{array}{lr}\text { SWH } & 9009.009 \mathrm{~Hz} \\ \text { FIDRES } & 0.274933 \mathrm{~Hz} \\ \text { AQ } & 1.8186240 \mathrm{sec} \\ \text { RG } & 135.71 \\ \text { DW } & 55.500 \text { usec } \\ \text { DW } & 7.62\end{array}$

$\begin{array}{lr}\text { TE } & \begin{aligned} 7.62 \\ \text { TE }\end{aligned} \\ \text { D1 } & 2.00000000 \mathrm{~K} \\ \text { TD } & \end{array}$

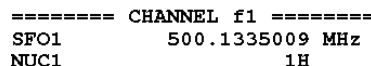

$\begin{array}{lr}\text { P1 } & 1 \mathrm{H} \\ \text { PLW1 } & 19.95299911 \mathrm{~W}\end{array}$

F2 - Processing parameters

$\begin{array}{lc}\mathrm{SI} & 32768 \\ \mathrm{SF} & 500.1300212 \mathrm{MHz}\end{array}$

WDW $500.1300212 \mathrm{MM}$

$\begin{array}{lll}\text { SSB } & 0 & \\ \text { LB } & 0 \mathrm{~Hz} & \\ \text { GB } & 0 & \\ \text { PC } & & 1.00\end{array}$

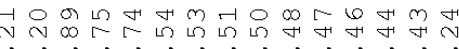

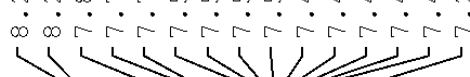
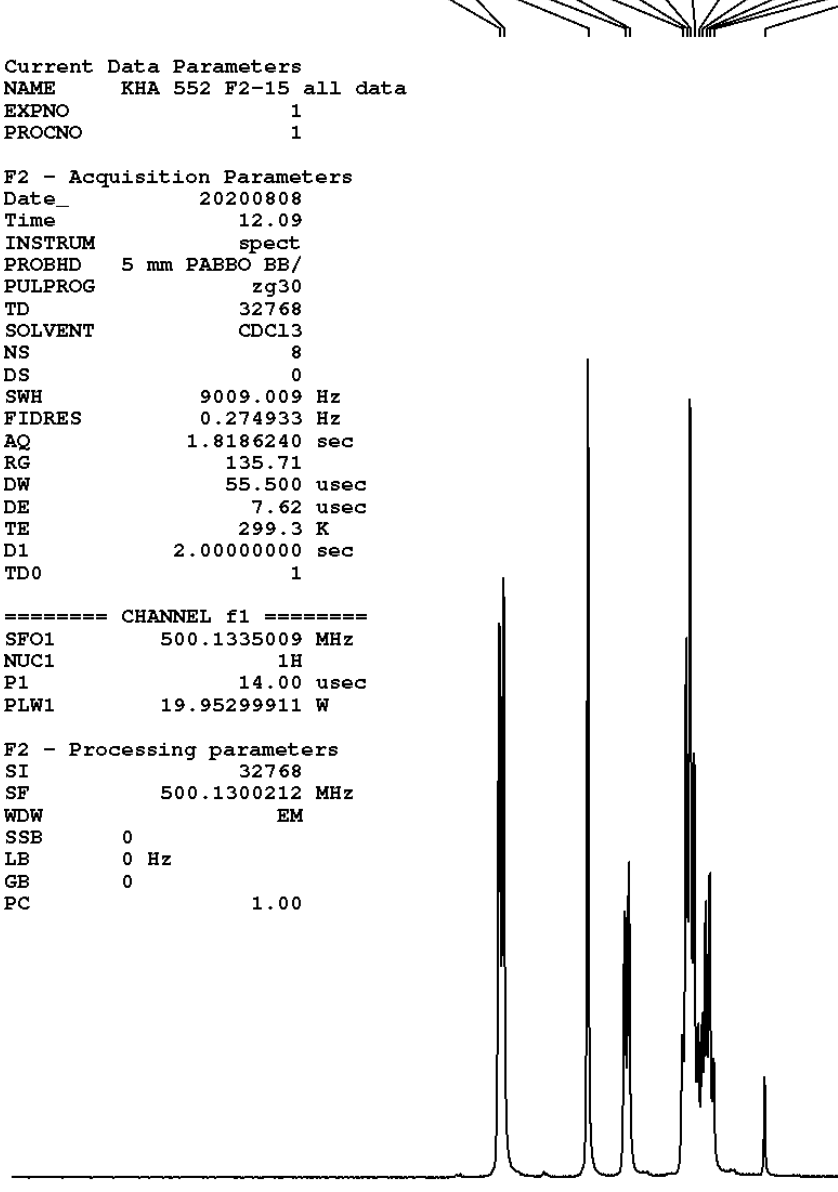

Figure S86. ${ }^{1} \mathrm{H}$ NMR spectrum of compound $5 \mathbf{a}\left(500 \mathrm{MHz}, \mathrm{CDCl}_{3}, 25{ }^{\circ} \mathrm{C}\right)$ 
Current Data Parameters
Nevg KKa SS2 $72-15$ all dat.

EXPNO

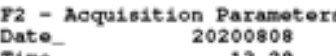

13.29
INPSTRLM

PROBHD
PULPROG

SOLVE

$\begin{array}{lc}\text { DS } & 4000 \\ \text { SWH } & \text { O } \\ \text { FID } & 28046.154 \mathrm{~Hz}\end{array}$

PIDRES $\quad 0.833415 \mathrm{~Hz}$

$\begin{array}{lr}A Q & 0.5999413 \\ R G & 200.35\end{array}$

17.333 usec
$\mathrm{DE}$

D1 $2.00000000 \mathrm{~K}$

TD1 $0.03000000 \mathrm{Bec}$

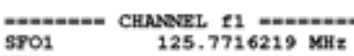

$\begin{array}{lr}\text { NFOC1 } & 125.7716219 \\ \text { P1 } & 13 \mathrm{C} \\ \mathrm{PDW} & 10.75 \mathrm{ue}\end{array}$

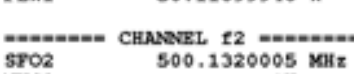

$\begin{array}{ll}\text { STC2 } & 500.1320005 \\ \text { CPDPRG } & 1 \mathrm{H}\end{array}$

$\begin{array}{ll}\text { CPDPRG 2 } & \text { waltz16 } \\ \text { PCPD2 } & 80.00 \text { usec }\end{array}$

PLW2 19.95299911 w

PLW1 $\quad 0.63826001$ W

92
SI - Processing paraneters
32768

WP $125.7577739 \mathrm{NLIx}$

$\begin{array}{llc}\text { WDW } & \text { EM } \\ \text { SSB } & 0 & 2.00 \mathrm{~Hz}\end{array}$

$\underset{\mathrm{PC}}{\mathrm{GB}} \quad 1.00$

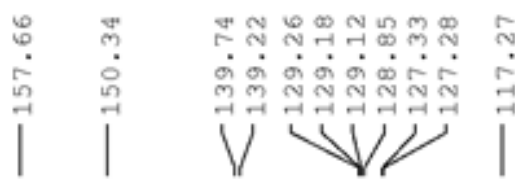

$$
1.00
$$

Figure S87. ${ }^{13} \mathrm{C}$ NMR spectrum of compound $5 \mathbf{a}\left(125 \mathrm{MHz}, \mathrm{CDCl}_{3}, 25{ }^{\circ} \mathrm{C}\right)$ 


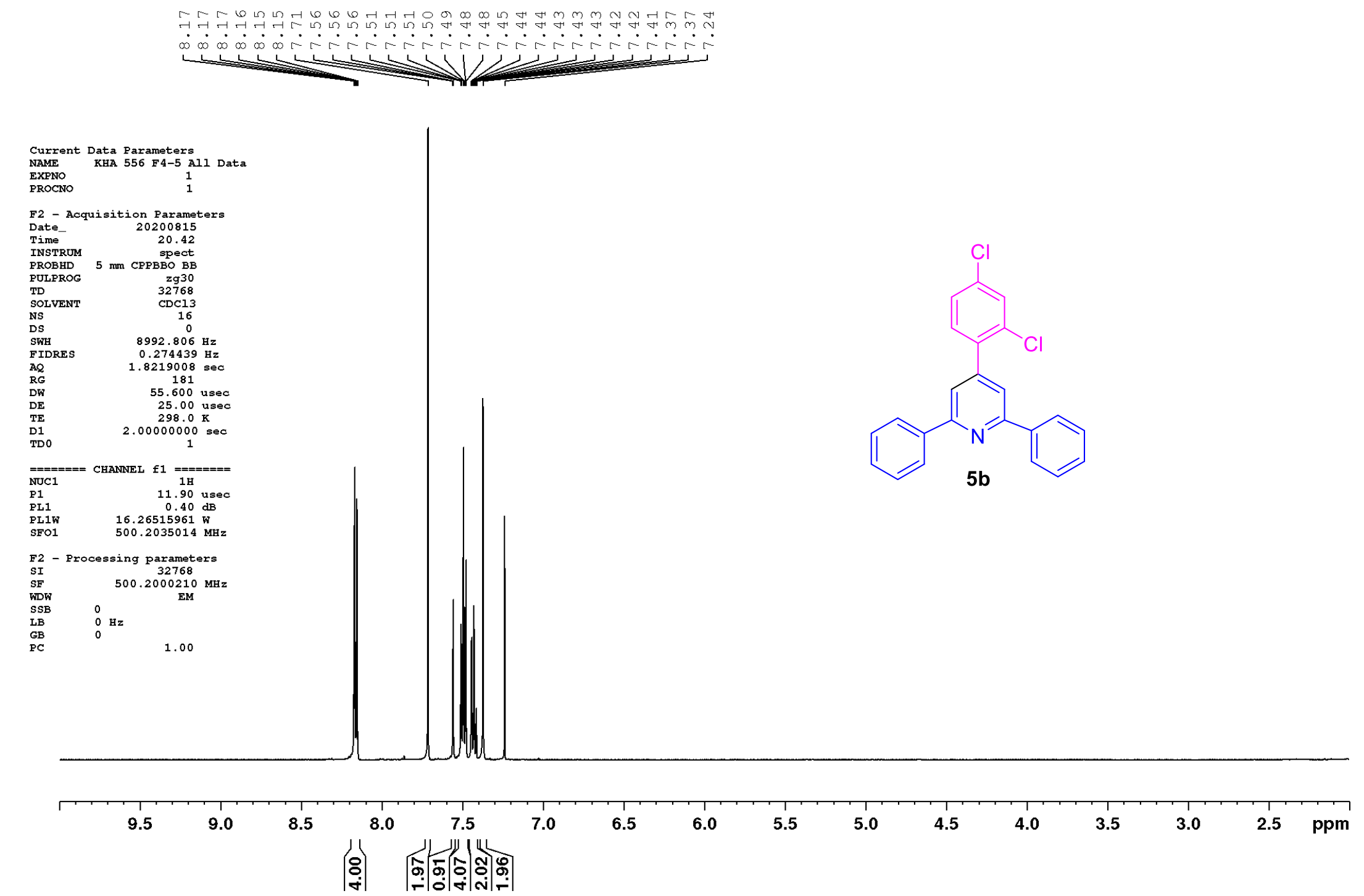

Figure S88. ${ }^{1} \mathrm{H}$ NMR spectrum of compound $\mathbf{5 b}\left(500 \mathrm{MHz}, \mathrm{CDCl}_{3}, 25{ }^{\circ} \mathrm{C}\right)$ 


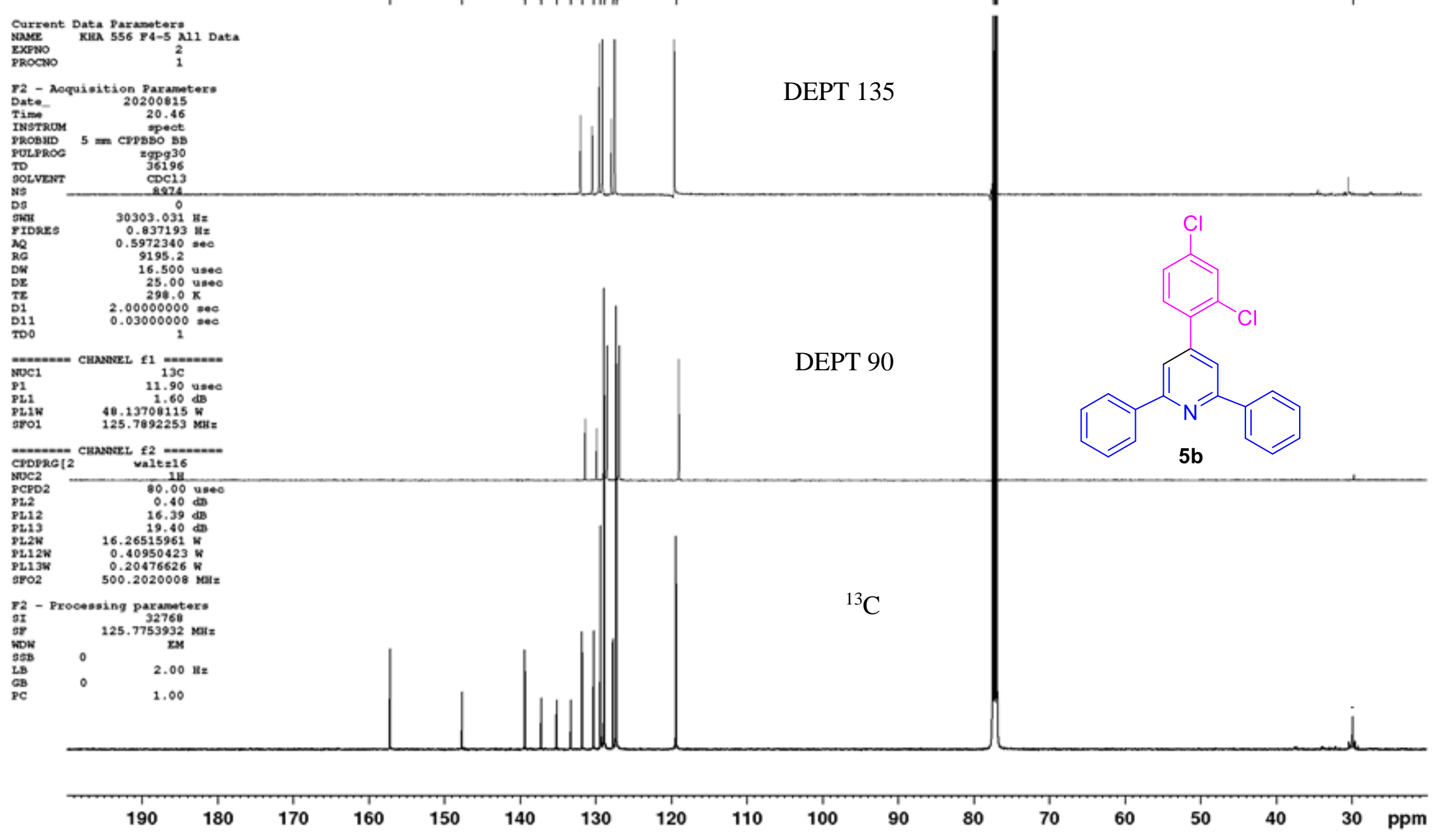

Figure S89. ${ }^{13} \mathrm{C}$ NMR spectrum of compound $\mathbf{5 b}\left(125 \mathrm{MHz}, \mathrm{CDCl}_{3}, 25{ }^{\circ} \mathrm{C}\right)$

S-131 

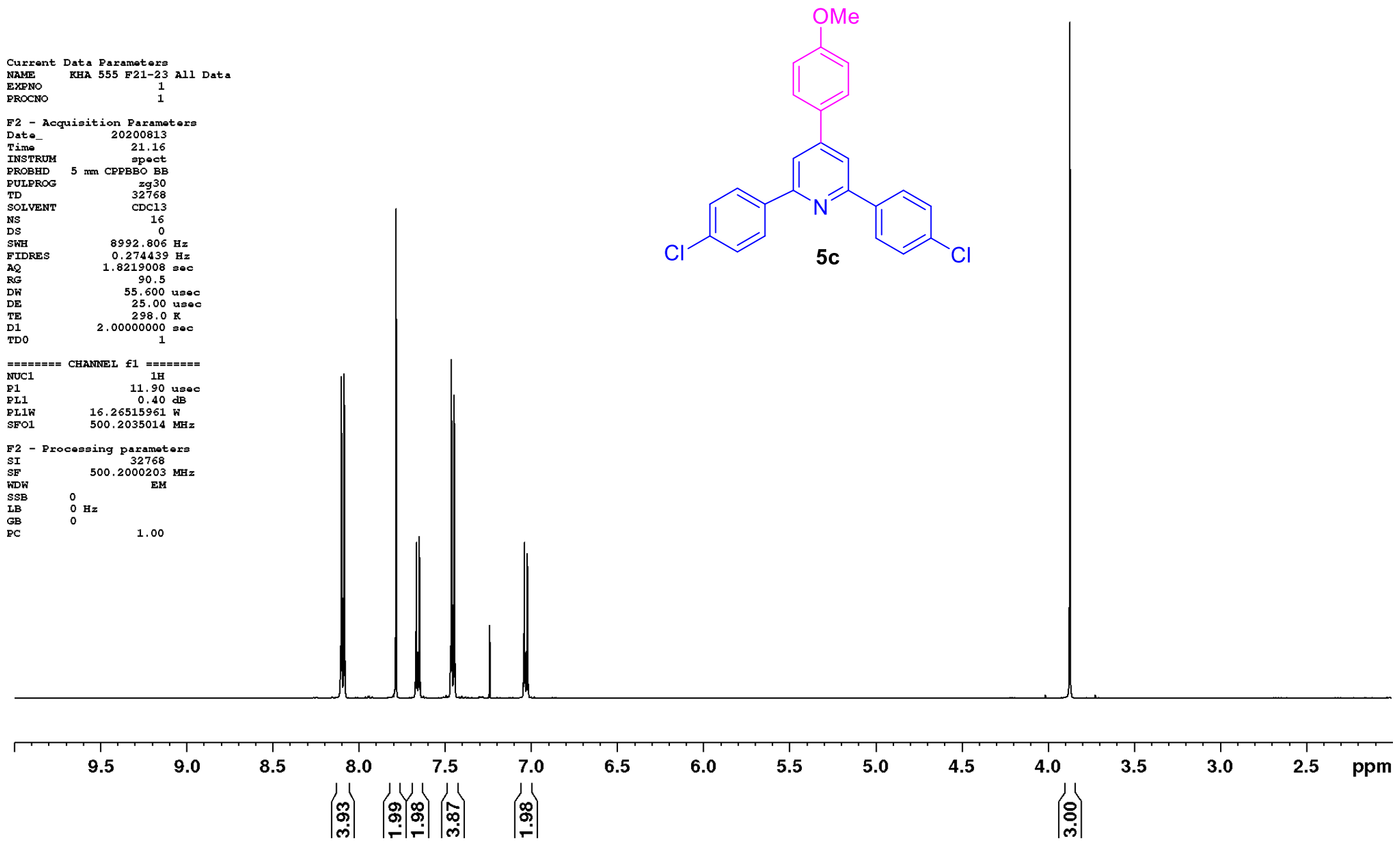

Figure S90. ${ }^{1} \mathrm{H}$ NMR spectrum of compound 5 c $\left(500 \mathrm{MHz}, \mathrm{CDCl}_{3}, 25{ }^{\circ} \mathrm{C}\right)$ 


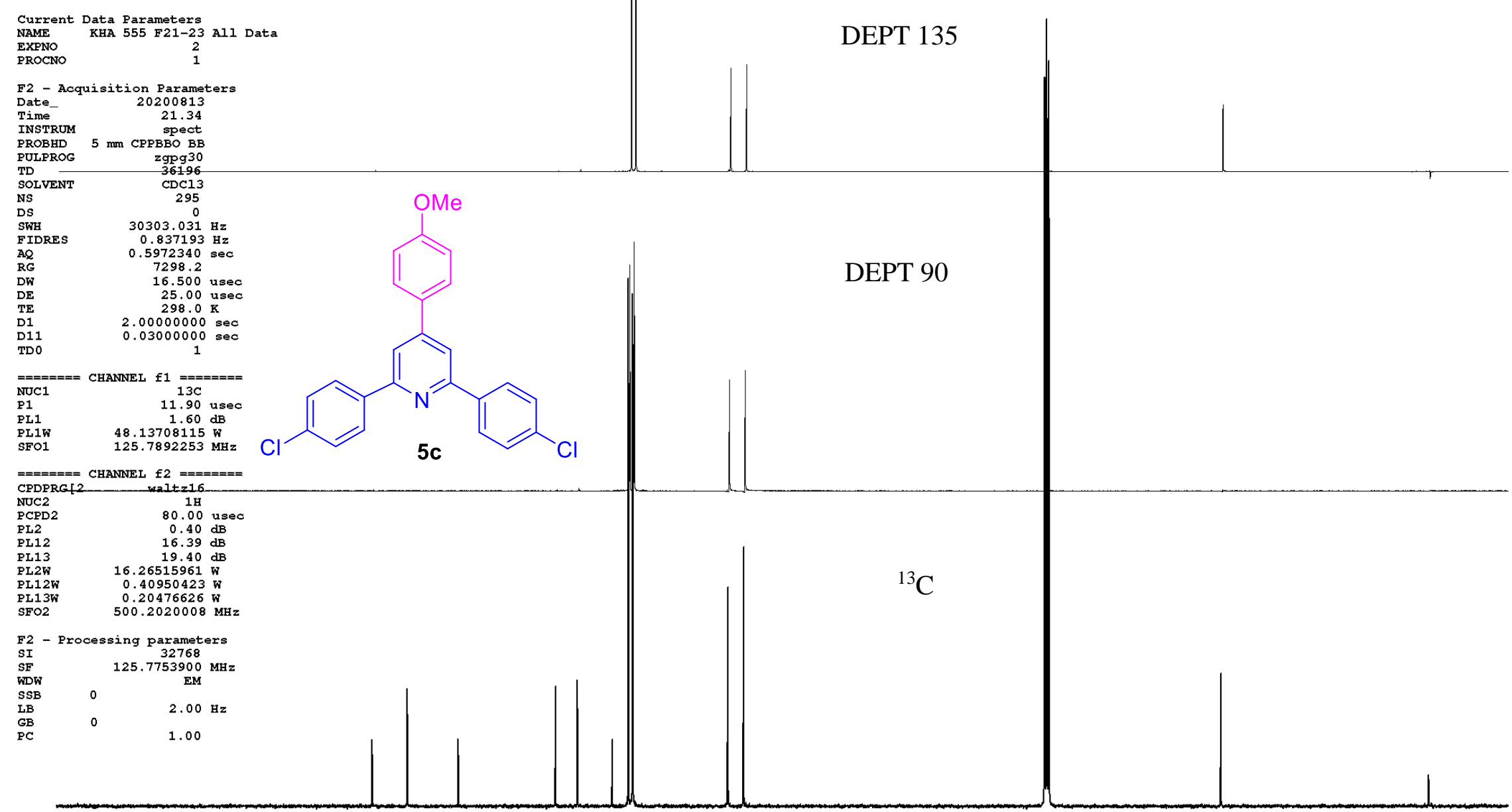

Figure S91. ${ }^{13} \mathrm{C}$ NMR spectrum of compound $\mathbf{5 c}\left(125 \mathrm{MHz}, \mathrm{CDCl}_{3}, 25{ }^{\circ} \mathrm{C}\right)$ 
Current Data Parameters
NAME KHA 553 F18-22All Data

EXPNO 1
1

F2 - Acquisition Parameters

$\begin{array}{lr}\text { Date } & 20200810 \\ \text { Time } & 21.30\end{array}$

INSTRUM

PROBHD

PULPROG

TD

SOL
DS
SWH

$\begin{array}{lr}\text { DS } & 16 \\ \text { SWH } & 0\end{array}$

$\begin{array}{ll}\text { SWH } & 8992.806 \mathrm{~Hz} \\ \text { FIDRES } & 0.274439 \mathrm{~Hz}\end{array}$

$\mathrm{AQ} \quad 1.8219008 \mathrm{sec}$

71.8
55.600 use

$\begin{array}{lr}\text { DE } & 25.00 \text { usec } \\ \text { TE } & 298.0 \mathrm{~K} \\ \text { D1 } & 2.00000000 \mathrm{sec} \\ \text { TD0 } & 1\end{array}$

TDO

$\begin{array}{ll}=======\text { CHANNEL } \mathrm{f} 1 \mathrm{l}======= \\ \text { NUC1 } & 1 \mathrm{H} \\ \text { P1 } & 11.90 \text { usec } \\ \text { PL1 } & 0.40 \mathrm{~dB}\end{array}$

$\begin{array}{lr}\text { PL1 } & 0.40 \mathrm{~dB} \\ \text { PL1W } & 16.26515961 \mathrm{~W}\end{array}$

F2 - Processing parameters

$\begin{array}{ll}\text { SI } & 32768 \\ \text { SF } & 500.2000206 \mathrm{MHz}\end{array}$

EM

$\begin{array}{llll}\mathrm{SSB} & 0 & \\ \mathrm{LB} & 0 & \mathrm{~Hz} & \\ \mathrm{~GB} & 0 & \\ \mathrm{PC} & & 1.00\end{array}$

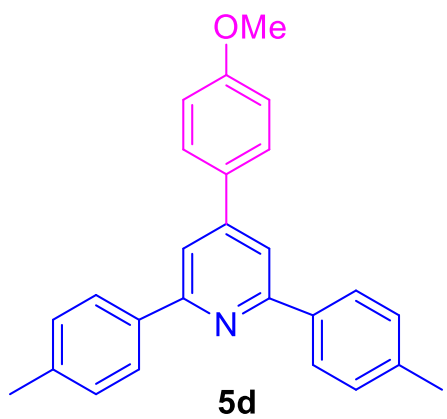

$5 d$

1.00

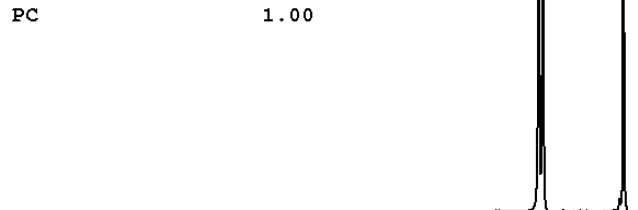

9.5

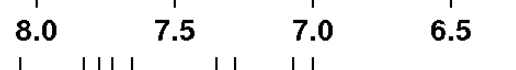

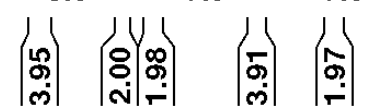

$6.0 \quad 5.5$

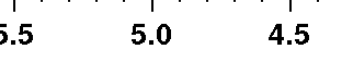

$\left|\begin{array}{l}8 \\ \dot{p}\end{array}\right|$

$.5 \mathrm{ppm}$

Figure S92. ${ }^{1} \mathrm{H}$ NMR spectrum of compound $\mathbf{5 d}\left(500 \mathrm{MHz}, \mathrm{CDCl}_{3}, 25^{\circ} \mathrm{C}\right)$ 


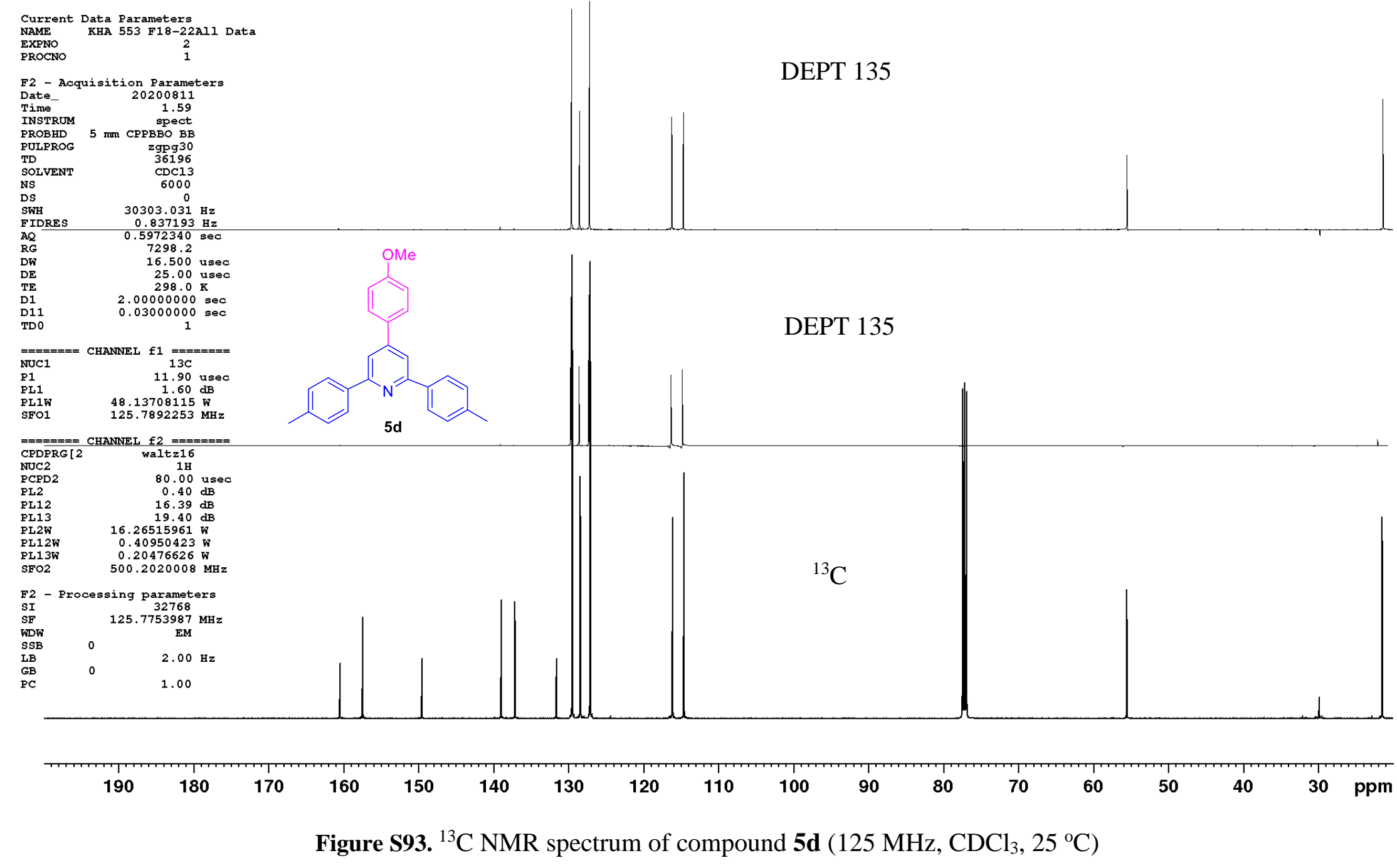



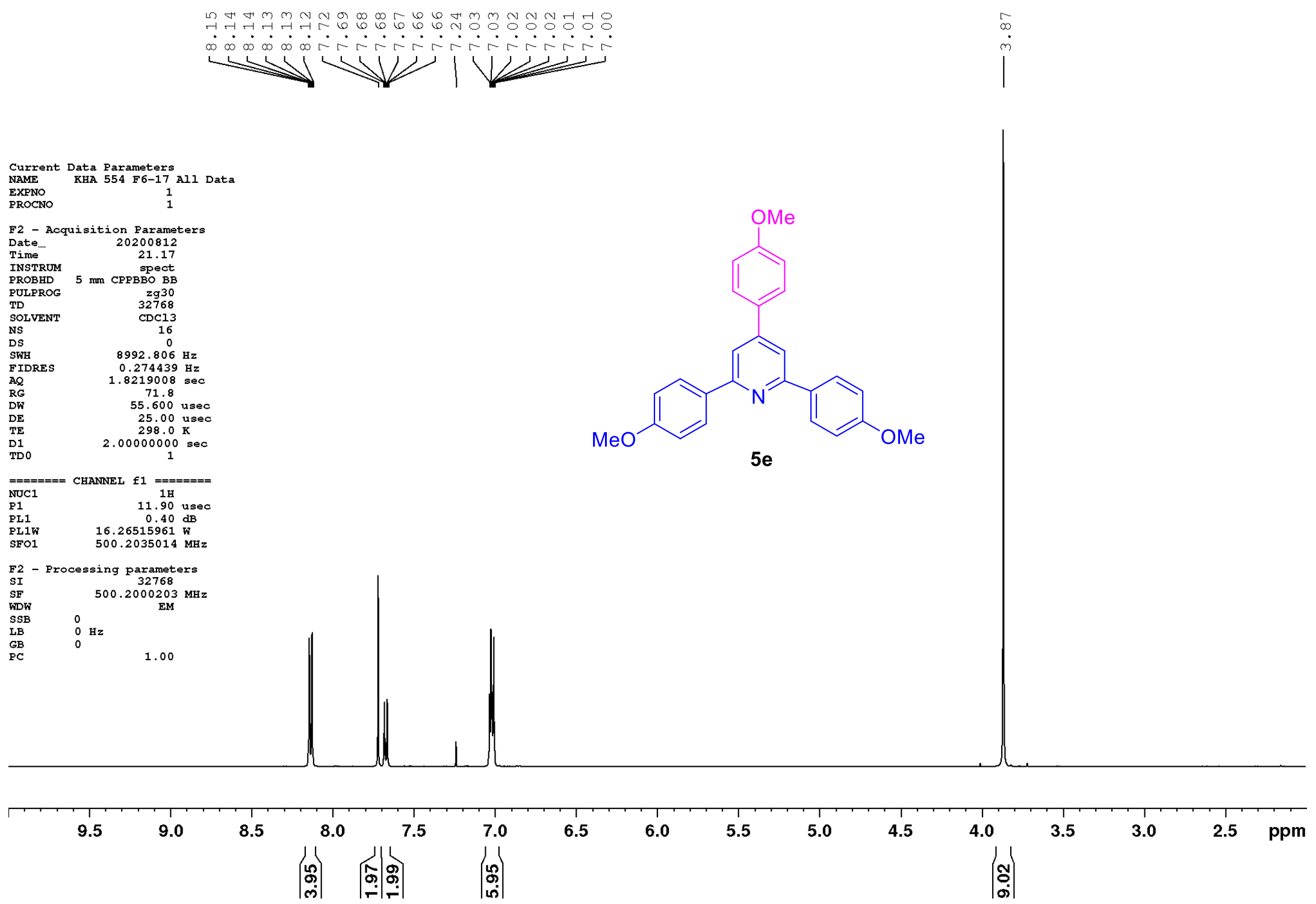

Figure S94. ${ }^{1} \mathrm{H} \mathrm{NMR}$ spectrum of compound 5 e $\left(500 \mathrm{MHz}, \mathrm{CDCl}_{3}, 25{ }^{\circ} \mathrm{C}\right)$ 


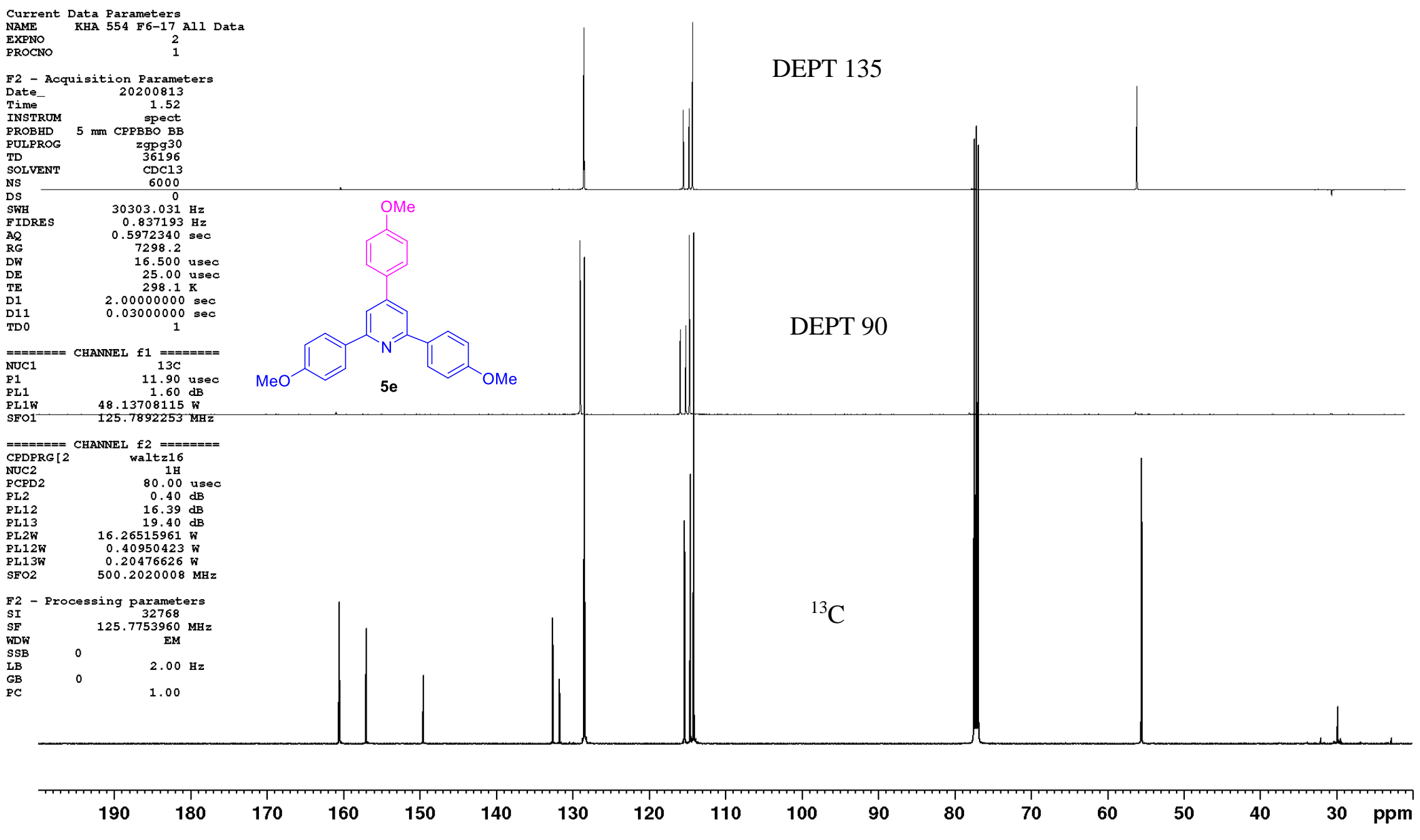

Figure S95. ${ }^{13} \mathrm{C}$ NMR spectrum of compound $5 \mathbf{e}\left(125 \mathrm{MHz}, \mathrm{CDCl}_{3}, 25{ }^{\circ} \mathrm{C}\right)$ 